Universidade de São Paulo

Instituto de Física

\title{
CONDIÇÕES DE CONTORNO MAIS GERAIS NO ESPALHAMENTO DE AHARONOV-BOHM DE UMA PARTÍCULA DE DIRAC EM DUAS DIMENSÕES: CONSERVAÇÃO DA HELICIDADE E DA SIMETRIA DE AHARONOV-BOHM
}

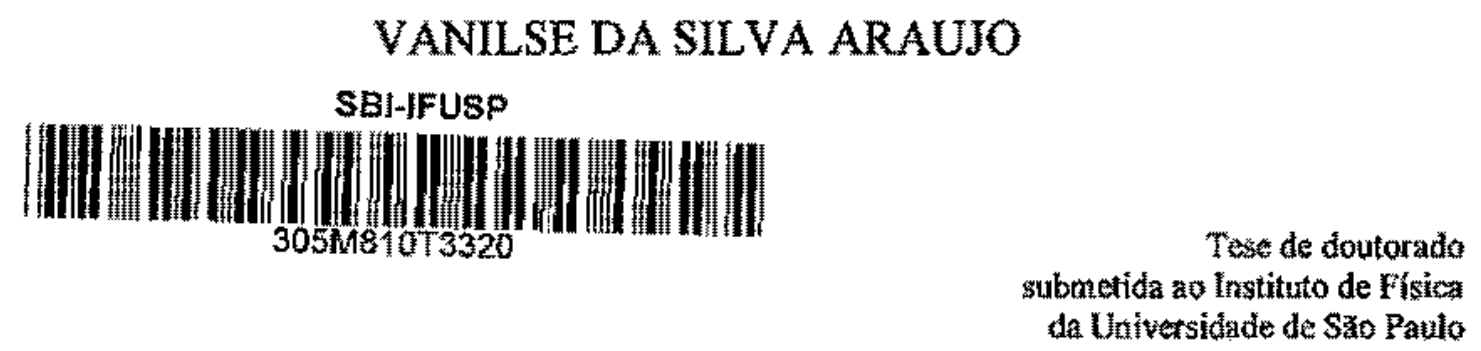

Banca examinadora:

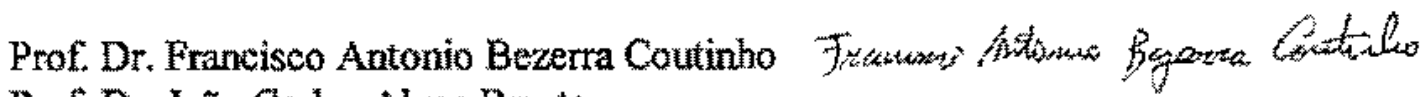

Prot. Dr. Joâo Carlos Alves Barata

Prof. Dr. Marcelo 0. Caminha Gomes

Prof. Dr. Henrique von Dreifus

Prof. Dr. Amir O. Caldeira

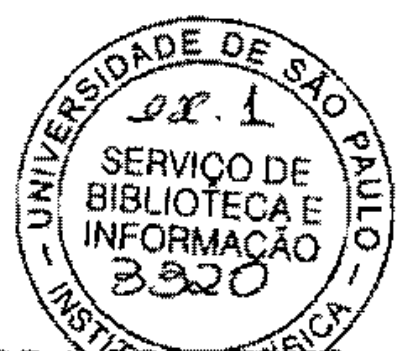

ORIENTADOR: Prof. Dr. FRANCISCO ANTONIO BEZERRA XOU]TUT CO-ORIENTADOR: Prof. Dr. JOSÉ FERNANDO PEREZ

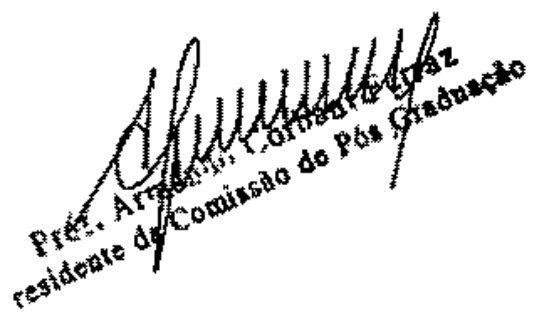

São Paulo, 2000. 


\section{FICHA CATALOGRÁFICA}

Preparada pelo Serviço de Biblioteca e Informação do Instituto de Fisica da Universidade de São Paulo

Araujo, Vanilse da Silva

Condiçōes de contorno mais gerais no espalhamento Aharonov-Bohm de uma partícula de Dirac em duas dimensōes: conservaçäo da helicidade e da simetria de Aharonov-Bohm. Säo Paulo, 2000.

Tese (Doutorado) - Universidade de São Paulo. Instituto de Fisica - Departamento de Física Matemática.

Orientador: Prof. Dr. Francisco Antōnio Bezerra Coutinho

Área de Concentraçāo: Física

Unitermos: 1. Efeito Aharonov-Bohm; 2. Extensobs Auto-adjuntas; 3. Condiçoes de contorno.

USPIIF/SBI-026/2000 
Dedico essa 1ese a quem faz parte de winha história $e$ me ensina a escrevê-la com doçura e confianca.

A quem inspira em meu coraçáto o amor de Deus e a Vida e me fazendo olhar para as lirios do campo e para as aves do céu, transforma os problemas em meros detalhes a serem sempre superados.

A quem Inmina men caminho com o sen sorriso $e$ me empresta sua coragem, pondo seus bragas a disposiçäo.

Dedico a quen en amo: 
- $\quad t^{2} 91$ sotquastadd "Aoymas op wan solqpI sop muas pisodsas 0 smu 'soupld lazw apod wauoy op opsosos $O$, 


\section{Agradecimentos}

Agradeço a CAPES pelo apoio financeiro.

Agradeço ao Prof. Coutinho e ao Prof. Perez, pessoas que, antes de tudo, admiro pelo indiscutivel brilhantismo acadêmico.

Particularmente ao Prof. Coutinho, agradeço pela sugestão do tema, por compartilhar seus conhecimentos, pela orientação que permitiu meu desenvolvimento científico e também meu desenvolvimento pessoal, impondo-me maior autoconfiança.

Agradeço ao Prof. Perez pela co-orientação, por sua positividade, pelas discussốes imprescindiveis nos vários momentos críticos desse trabalho.

Agradeço também aos Profs. Coutinho e Perez pela compreensão, pelo apoio em diversas situaçôes e pelo bom humor constante que sempre me contagiou.

Agradeço ao Prof. Barata pelo auxilio norteador, pelas sugestôes enriquecedoras e pela apreciação do artigo.

Agradeço a todos os meus amigos que contribuiram direta ou indiretamente e em especial ao Prof. Cavallari e ao Prof. Guidorizzi.

Agradeço a minha saudosa mâe Abigail e ao meu querido pai José pelos seus esforços sem limites, que permitiram minha formação humana e acadêmica e por seus testemunhos de vida, que sempre me inspiraram na busca de meus ideais.

Agradeço ao meu amigo Tadeu por ser meu irmão muito querido e com quem sempre pude contar.

Agradeço aos familiares do Jefferson: Dna. Ile, tia Vilma, Fabiana, César, Andrea e André pela generosidade e pelo zelo com o qual sempre me cercaram, pelo incentivo e pelo apoio que me deram.

Agradeço ao Jefferson, dádiva maravilhosa do Senhor en minha vida, por seu amor, paciência e sabedoria e por estar sempre junto, abraçando com ternura e vontade as "nossas causas".

Agradeço a Deus por tudo. 


\section{Resumo}

Nessa tese, mostramos que a Hamiltoniana $H$ e o operador helicidade $A$ de uma partícula de Dirac que se movimenta em duas dimensōes na presença de um tubo de fluxo magnético infinitamente fino na origem admitem, cada um, uma familia de quatro parânetros de extensōes auto-adjuntas. Para cada extensäo correspondem condiçôes de contorno a serem satisfeitas pelas auto-funçoes na origem. Apesar dos operadores $H$ e $\mathrm{A}$ formalmente comutarern antes da especificaşa das condigües de contorno, para garantirmos a conservaçẫo da helicidade, nầ é suficiente obtermos as mesmas condiçōes de contorno para ambos os operadores, ou seja, näo é suficiente a determinaçāo de um domínio comum a ambos. Mostramos que, para certas relaçōes entre os parämetros das extensōes satisfeitas , é possivel a determinação dos domínios mais gerais onde ambos os operadores $H$ e $A$ săo auto-adjuntos e onde a helicidade é conservada, simultaneamente com a preservaça da simetria de Aharonov-Bohm $(\phi \rightarrow \phi+1)$, onde $\phi$ e o fluxo magnêtico em unidades naturais. Nossos resultados implicam que, nem a conservaç艹o da helicidade nem a simetria de Aharonov-Bohm, resolvem o problema da scolha da condição de contorno "fisicamente correta ". 


\begin{abstract}
We show that both the Hamiltonian $H$ and the helieity operator $A$ of a Dirac particle moving in two dimension in the presence of an infinitely thin magnetic fux tube admit each a four-parameter family of self-adjoint extensions. Each extension is in one-to-one correspondence with the boundary conditions (BC's) to be satisfied by the eigenfunctions at the origin. Although the actions of these two operators commute before specification of boundary conditions, to ensure helicity conservation it is not sufficient to take the same $\mathrm{BC}$ 's for both operators. We show that, given certain relations between the parameters of the extensions it is possible to write down the most general domain where both operators $H$ and $A$ are selfadjoint with helicity conservation and aiso Aharonov-Bohm symmetry $(\phi \rightarrow \phi+1)$ preserved, where $\phi$ is the magnetic thx in natural units. The continuity of the dymarnics is also obtained. Our results imply that neither helicity conservation nor Aharonov-Bohm symratry by themsalves solves the problem of choosing the "physical "boundary conditions for this system.
\end{abstract}




\section{Índice}

Introdução.................................................................... 1

Capítulo 2 - Os operadores $H$ e $\Lambda$ e a necessidade de extensões auto-adjuntas......................................... 5

2.1. Definiçöes formais dos operadores $H$ e A ................................ 5

2.2. Determinaçäo das auto-funçöes mais gerais para o operador Hamiltoniana............................................................................. 9

2.3. Determinaçāo das auto-funçöes mais gerais para o operador Helicidade.

2.4. O caso Livre................................................................................ 18

Capítulo 3 - Um Método Heurístico para verificação da necessidade de extensões auto-adjuntas............... 23

Capítulo 4 - $O$ Método de von Neumann para obtenção das extensões auto-adjuntas....................... 26

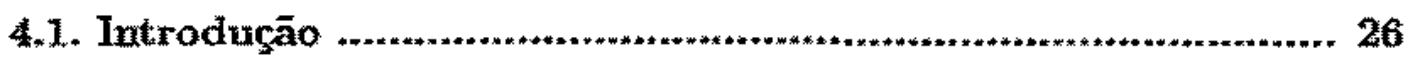

4.2. Algumas definiçōes.................................................................. 27

4.3. Critérío para possibilidade de extensöes auto adjuntas........... 30

4.4. Técnica dos índices de deficiência de von Neumann para obtenção das extensöes auto-adjuntas de um operador simétrico

4.5. Relação entre as extensöes auto-adjuntas e as extensōes de contorno.

4.6. Obtençăo das extensöes auto-adjuntas para o operador momento linear em uma dimensäo. 
4.7. Obtengāo das extensöes auto-adjuntas para o operador energia cinética definido em $L^{2}(0, \infty)$ em uma dỉmensẵo........ 35

4.8. Obtengāo das extensöes auto-adjuntas para o operador energia cinética defintido em $t^{2}(-\infty, \infty)$ em uma dimensăo

Capítulo 5 - Extensôes autom adjuntas a dois parâmetros que não acoplam todas as componentes das funçöes de onda.................................................... 43

5.1. Extensōes auto- adjuntas para os operadores $H$ e A............. 43

5.2. Extensöes auto adjuntas para o operador $H$ - Um Caso Particular

5.3. Condiçöes de contorno mais gerais admissiveis para o operador $\Lambda$

*.4. Condiçôes de contorno e Conservaçāo da Helicidade. 61

5.5. Determinaģäo das auto-funçōes do operador $H$ que satisfazem as condiçôt de contorno desacopladas.

5.6. Determinaçäo das auto-funçōes do operador $\Lambda$ que satisfazem as condicōes de contorno desacopladas.

5.7. Discassäo dos Resultados. 65

5.8. Determinagāo da condigāo formal para que os operadores H e $A$ tenharm auto-funçöes comums.

5.9. Auto-funçồes simmultâneas para os operadores $H$ e $\Lambda$ no domínio $D_{\delta}(H, \Lambda)$ dass condiçōes de contorno desacopladas..... 68

5.10. A ação do operador $A$ nas auto-fumçōes de $H$ definidas em $D_{\delta}(H, \Lambda)$ 
Capítulo 6 - Extensōes auto-adjuntas mais gerais a dois parâmetros para o operador $H$ e conservação da helicidade.

6.1. Determinaçäo das condiçöes de contorno

6.2. Determinaçäo das auto-funçôes de $H$ que satisfazem as condiçöes de contorno mais gerais a dois parâmetros.

Capítulo 7 - Extensōes auto-adjuntas a três parâmetros para o operador Hamiltoniana e conservaçäo da helicidade............................................ 80

7.1. Determinaçäo das condiçōes de contorno.................................... 80

7.2. Determinação das auto-funçōes.................................................. 82

7.3. Verificando se $\Lambda$ é auto-adjunto no domínio $D_{0, \psi, \psi}^{*}(H, \Lambda)$ encontrado................................................................................... 87

7.4. Exemplo: Uma extensảo particular a três parâmetros............. 91

7.5. Verificando se o operador $\Lambda$ é auto-adjunto no domínio $D_{x, 0, \frac{3 x}{4}}^{\gamma}\left(H_{2} \Lambda\right)$

Capítulo 8 - Extensões auto-adjunta mais gerais a quatro parâmetros para o operador $H$ e conservação da helicidade.

8.1. Determinação das condiģôes de contorno mais gerais com quatro parâmetros para o operador Hamiltoniana

8.2. Determinaçäo das auto-funçöes de $H$ que satisfazem as condiçôes de contorno mais gerais com quatro parầmetros...... 98

8.3. Verificando se $A$ é auto-adjunto no dominio mais geral $D_{\Psi, \alpha, t, p}^{\prime}(H)$ encontrado 
Capítulo 9 - Simetria de Aharonov-Bohm e conservaçäo da helicidade......................................... 107

Capítulo 10 - Determinaçăo das condiģōes de contorno a partir de um procedimento limite.

10.1. Introduçāa - Procedimento Limite............................................. 125

10.2. Modelo do Tubo de Fluxo.................................................... 127

10.3. Processo limite para a obtenção de todas as extensöes a um parâmetro.................................................................................. 133

Capítulo 11 - Conclusões......................................... 136

Apêndice A - ...................................................... 138

Apêndice $B$ - ............................................. 140

Apêndice $C$. ...................................................... 143

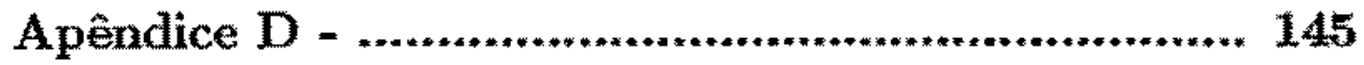

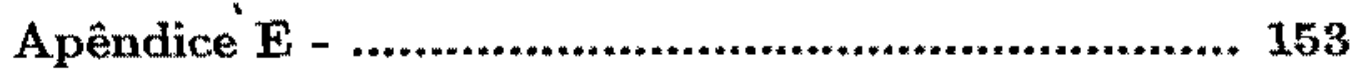




\section{Capítulo 1 Introdução}

O problema de uma partícula de Dirac que se move num plano na presença de um tubo de fluxo magnético infinitamente fino na origem aparece em vários problemas diferentes em física teórica. Originalmente, esse problema apareceu na discussão do efeito Aharonov-Bohm ${ }^{[15,16]}$ e de sua variante, o efeito AharonovCasher ${ }^{[17]}$. Aparece também no estudo da interação da matéria com cordas cósmicas ${ }^{\left[{ }^{18}-20\right]}$. No limite não relativístico, o problema de uma partícula em duas dimensōes na presença de um tubo de fluxo magnético aparece no estudo da supercondutividade a altas temperaturas críticas ${ }^{[21]}$.

O problema de uma partícula de Dirac de massa $m>0$, que se move num plano na presença de uma linha de fluxo magnético na origem, já foi considerado na literatura por vários autores ${ }^{[1-7,16,22]}$.

Foi mostrado pela primeira vez por $\mathrm{Ph}$. de Sousa Gerbert ${ }^{[3]}$ que, diferentemente do caso de uma partícula de Dirac livre em duas dimensões, a introdução da linha de fluxo magnético na origem torna o problema de determinação das auto-funções do operador hamiltoniana bem mais complicado. Isto porque, o operador hamiltoniana passa a requerer, para sua completa determinação como um operador auto-adjunto, a especificação de condiçōes de contorno a serem satisfeitas pelas funçōes de onda na origem ${ }^{[1,2,3]}$.

No entanto, tecnicamente falando, o problema de determinação das condiçóes de contorno admissíveis é idêntico ao problema de determinação das extensōes auto-adjuntas para um operador simétrico definido num domínio denso e fechado. Assim, o autor Sousa Gerbert encontrou uma família de extensões auto-adjuntas para o operador hamiltoniana ${ }^{[3]}$. Posteriormente, os autores F. A. B. Coutinho e J. Fernando Perez ${ }^{[1]}$ demonstraram que o operador hamiltoniana admite uma familia de extensōes auto-adjuntas a quatro parâmetros e encontraram as extensōes auto-adjuntas a dois parâmetros para o operador hamiltoniana, com a restrição de que esse operador continuasse possível de ser decomposto em dois operadores desacoplados atuando en espinores de duas componentes. Desse modo aparece uma indeterminação na escolha das condiçōes de contorno, sendo possiviveis várias dinâmicas diferentes. Surge, então, naturalmente a questão de se saber qual dinâmica é a "fisicamente correta".

Foi sugerido por Hagen ${ }^{[5,6]}$ que a dinâmica "fisicamente correta" seria aquela obtida a partir de um processo limite onde se toma um tubo de fluxo magnético de 
raio $R>0$ (onde o campo ruagnético $\vec{H}$ é dado por uma fungäo do tipo delta de cusca), removendo-se assim a ambiguidade e depois tomando-se o limite $R \rightarrow 0$ no final dos cálculos. Esse procedimento também é utilizado nos cảloulos das referências [2], [4], [5], [6] e [7]. As dinämicas assim obtidas ${ }^{[2,4-7]}$ conservam todas elas a helicidade e segundo Hagen, seriarn as fisicamente corretes,

Poderímos pensar na conservação da helicidade como sendo a motivaçāo física para a escolha das condiçoes de contorno dentre as possiveis, ramovendo assim a indeterminaça existente.

Os autores F. A. Coutinho e J. Femando Perez no artigo da referéncia [1] concluen que existe mats de uma dunámica possivel que preserva a helicidade (na verdade uma família de extensöes auto-adjuntas que não acoplam as quatro componentes das funcōes de onda). De modo que a conservaçầ da helicidade näo removeria a indetermínaçào na escolha das condigôes de contorno.

$\mathrm{Na}$ verdade, como discutiremos nessa tese, algumas dag aftrmaçöes feitas na referência [1] nào estão corretas. Surprecudentemente, apenas a comutação formal entre os operadores hamiltoniana e helicidade e a definição de um domínio comum onde ambos säo operadores auto-adjuntos näo é suficiente para garantir a comutação efetiva entre esses operadores e a consequente obtencäo de antofunçoes comuns. De fato, é possivel a conservaçäo da helicidade, apenas para cortas extensốs auto-ady̆untras a $\mathrm{um}$ (ou dois) parâmetros que nāo acoplam as quatro componertes, como veremos nessa tese.

Outra importante simetria que aparece nesse problema è simetris de AharonovBohm, que consiste na invariancia do opexador hamiltoniana através da transtormação $\rightarrow \phi+1$, onde $\phi$ to fluxo magnetico. As condiçôs de contorno que aparecem nas referências $[2],[4],[5],[6]$ e $[7]$, embora conservem a helicidade, victam a simetria de Aharonoy-Bohm.

$\mathrm{Na}$ referência [2] foi demonstrado que é possivel através de uma interpolação resstabelecer a simetria de Aharonow Bohm, mas, nesse caso, näo é mais possivel a conservação da helicidade. Assim, năo aparece na litheratura dinămicas onde ambes as simetrias sejam preservadas.

Nesse trabalho, encontramos as extensobs anto-adjuntas mais gerais a quatro parâmetros para o operaclor hamiltoniana, ques, queplam as quatro componentes das funçöes de onda, que pernitem várias dinâmicas diferentes onde ambas as simetrias: conservacha da helicidade e AharonovmBohm, são preservadas.

Essa tese está organizada do seguinte modo. No Capítulo 2 apresentamos a definiça formal dos operadores hamiltoniana $H$ e helicidade $\Lambda$, e mostramos que eles comutam formalmente. Encontramos as auto-funçoes mais gerais de 
cada um desses operadores e verificamos através da análise destats auto-funcóes a necessidade de extensöes auto-adjuntas para $H$ e $\Lambda$ quando $\nu=n+\phi(n$ e momento angular orbital e $\phi$ é o fluxo magnético) está no intervalo $v:]-1,0[$.

No Capitulo 3, apresentamos um método hetrístico para se verificar a necessidade de extensós auto-adjuntas para $H$ $H$, que consiste em se kutalisar a divergênctia e considerar os termos dominantes nas exuaçöes diferenciais que definem as actoes dos operadores $H$.

No Capítulo 4, revisamos alguns dos principais resultados da teoria de von Neum mann dex extensöes auto-adjuntas e apresentanos dois exemplos, onde aplicamos a técrica dos indices de defictência para obtermos as extensós auto-adjuntas. $O$ primeiro exemplo mais simples é do operador momento linear definido en $L^{2}(0,1)$. O segundo exemplo é o do operador energia cinética definido em $l^{2}(-\infty, \infty)$, cujas extensões auto-adjuntas jh foram obtidas na literatura ${ }^{[8,13,14]}$. Dernonstrsmos que a técnica que utilizamos para obtermos as extensōes suto-adjuntas ê capaz de reproduzir os mesmos resultados das referênciass [8], [13] e [14].

No Capítulo 5, passamos ao problema de determinaça das extensöes antoadjuntas para os operadores $H$ e $A$, e nos concentramos no caso particular tratado na referêneia [1], de extensôes auto-adjuntas a dois parâmetros que nểo acoplam as quatro componentes das funçoes de onda. Mostramos que apesar dos operadores $H$ e $A$ comutarem formalmente, a definigs de um domínio comum, onde ambos sâo auto-adjuntos, näo garante a comutagäo efetiva desses operadores como erroneamente se afrmou na referéncia [1], Lasso ocorre porque a imposigão da conservacăo da halicidade pode ser obtida a partir de uma condiçấo formal que só é satisfeita para certos valores dos parấnetros da extensäo. Assim, na verdade, a cornutacào efetiva desses operadores e conseqüuente obtencäo de anto-funçóes comuns só ocorre para certas extensöes particulares. As auto-finçöes cormus säo encontradas nesse caso e mostramos que elas inchem as auto-fungös obtidas nas referências [2], $[4],[5],[6] \mathrm{e}[7]$.

No Capítulo 6, obtemos as extensós auto-adjuntes mais gerais a dois paràmetros, essas que acoplam todas componentes das fungöes de onda. Verificamos que essas extensōes näs conduzem a novos resultados para as autó-funçōes, ou seja, não obtemos auto-funçöes diferentes das ja encontradas no Capítulo 5.

No Capitulo 7, procedemos a extensōes auto-adjuntas a três parâmetros para o operator $H$ e nesse caso veríficamos que é possível a conservação da helicidade, se certas relaçós entre os parâmetros da extensâo forem satisfaitas. Sendo possivel, assim, a definição de um dorninio comum onde arnbos os operadores $H$ e $A$ são auto-adjuntos e a helictade é conservada. 
No Capítulo 8, procedemos a extensäo auto-adjunta mais geral a quatro parâmetros que inchi todas as condiçōes de contorno apresentadas na literatura ${ }^{[1-7]}$ e verificamos que são possiveis várias dinämicas onde a helicidade ê conservada se certas relações entre os parâmetros da extenkão são satisfeitas.

No Capitulo 9, mostramos que as condicöes de contorno do Capítulo 8 (as condiçeres de contorno do Capitulo 8 obviamente, por serem as mais gerais, incluem as do Capítulo 7), além de preservarem a helicidade, preservam também a simetria de Aharonow Bohm, o que não se conseguia através das dinâmicas que aparecem nas referêneias [1] a [7]. Mostramos que, para certas escolhas do intervalo de variação dos parâmetros da extensäo, é possivel assegurar a simetria de AharonovBohm, através de uma interpolação.

No Capítulo 10, revisamos as cálculos e resultados da referência [2] e discutimos e comparamos os nossos resultados com os resultados anteriores que aparecern nas referêneias [2] a [7].

No Capítulo 11, apresentamos nossas conclusóts.

No Apêndice A, checamos explicitarxiente a comutação formal entre as operadores $H$ e $\Lambda$. No Apêndice $\mathrm{B}$, verificumos a relação de comutação entre os operadores $H$ e $J_{x}$ (momento angular total). No Apễndice $\mathrm{C}$, verificamos a relagăo de comutaçăo entre os operadores $\Lambda$ e $J_{z}$. No Apêndice $D$, discutimos e comparamos nossos resultados com os resultados das referências [5], [6] e[7]. No

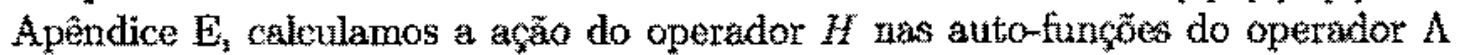
definidas no dominio do Capítnlo 5 e verificamos que $H$ não deixa tal domínio invariante. 


\section{Capitulo 2}

Os operadores $H$ e $\Lambda$ e a necessidade de extensōes auto-adjuntas.

\subsection{Definicöos Formaís dos Operadores $H$ $\Lambda$.}

O operatow hamiltoniana para uma partícula de Dirac de massa $m>0$, em duas dimensöes na presenç de um tubo de fluxo magnético infinitamente fino na origem é tormalmente dado por ${ }^{1,2 i}$;

$$
H=\vec{\pi} \cdot \vec{a}+\beta m
$$

onde:

$$
\vec{\pi}=\left(p_{x}+\frac{e}{c} A_{z}, p_{y}+\frac{e}{c} A_{y}\right)
$$

e:

$$
\vec{\alpha}=\left(\alpha_{1}, \alpha_{2}\right)
$$

onde una possivel escolha das matrizes de Dirac $\hat{\epsilon}^{\text {t }}$

$$
\begin{aligned}
& \alpha_{1}=\left[\begin{array}{cc}
\alpha_{2} & 0 \\
0 & \sigma_{1}
\end{array}\right] \\
& \alpha_{2}=\left[\begin{array}{cc}
\sigma_{2} & 0 \\
0 & \sigma_{2}
\end{array}\right]
\end{aligned}
$$

e:

$$
\beta=\left[\begin{array}{cc}
\sigma_{3} & 0 \\
0 & -\sigma_{3}
\end{array}\right]
$$

sendo $\sigma_{1}, \sigma_{2}$ e $\sigma_{3}$ as matrizes de Panli.

O potencial vetor $\vec{A}$ da equaçäo (2.1.2), está relacionado com o campo magnético $\vec{H}$ através da equação:

$$
\vec{H}=\vec{\nabla} \wedge \vec{A}
$$


Utilizando o gauge o Coulomb, o potencial vetor é dado por:

$$
\vec{A}=\frac{c}{e}\left(\frac{-y}{r^{2}}, \frac{x}{r^{2}}\right)
$$

onde $\phi$ é o fluxo magnético.

De modo que:

$$
\vec{H}=\vec{\nabla} \wedge \vec{A}=\left[\frac{2 \pi c}{e}\right] \frac{\delta(r)}{r} \vec{k}
$$

Podemos notar que o hamiltoniana definida pela equaçäo $(2.1 .1)$ pode ser decomposta em dois operadores atnando emr espinores de duas componentes:

$$
H=\vec{\pi} \cdot \vec{\sigma}+s m \sigma_{3}
$$

onde:

$$
\vec{\sigma}=\left(\sigma_{1}, \sigma_{2}\right)
$$

e:

$$
s= \pm 1 .
$$

As duas escolhas de $s$ levam a representaçōes nâo equivalentes das matrizes de Dirac. No limite năo relativistico para $s=+1$ apenas a componente superior do tspinor de duas componentes sobrevive, descrevendo una partícula de Schrödinger de spin $\frac{1}{2}$, enquanto para $s=-1$, apenas a componente inferior do espinor de duas componentes sobrevive descrevendo uma partícula de Schrödinger de spin $-\frac{1}{2}$.

Usando a representaçăo de espinores de quatro componentes, podemos definir o operador helicidade, que da a projeçäo do momento $\overrightarrow{\vec{*}}=\vec{p}+\frac{e}{\vec{A}}$, na direção do spin, como sendo:

$$
\Lambda=\left[\vec{p}+\frac{e}{c} \vec{A}\right] \cdot \vec{\Sigma}
$$


onde $\Sigma$ co operador de spin:

$$
\overrightarrow{\Sigma_{i}}=\left(\Sigma_{1}, \Sigma_{2}\right)
$$

onde:

$$
\begin{aligned}
& \Sigma_{1}=\left[\begin{array}{ll}
0 & \sigma_{1} \\
\sigma_{1} & 0
\end{array}\right], \\
& \Sigma_{2}=\left[\begin{array}{cc}
0 & \sigma_{2} \\
\sigma_{2} & 0
\end{array}\right] .
\end{aligned}
$$

Os dois operadores $A$ e $A$, definidos respectivamente pelas equaços (2.1.1) e (2.1.13), comutam tormalmente como se pode verificar. Veja o Apêndice A. No entanto, essa "comutação formal "dos operadores não garante a possibilidade de

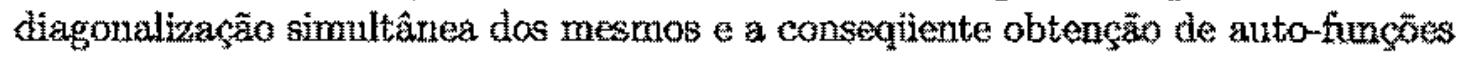
comuns. Ern outras palavras, a comutação formal unicamente näo garante a conservaçăo da helicidade para qualquer dinâmica dada por $H$.

Conforme ficará claro mais adiante, o problema reside no fato de os operadores H $A$ não serem exsencialmente auto-ajuntos para todo valor do fluxo magnético $\phi$, haveudo a necessidade de se especificar as condiçoes de contorno a serem satisfeitas pelas suas auto funçoes na origem, uma vez que, para certos valores do fluxo $\$$, năo é possível a imposiça usual de regularidade na origem.

Será bastante átil na solugho do problema de condiçồ de contorno de $\Lambda$, notar que existe uma transformąâa umitária $U$, dada por:

$$
U=\frac{1}{\sqrt{2}}\left[\begin{array}{cc}
I & -i \sigma_{3} \\
I & i \sigma_{3}
\end{array}\right],
$$

que conecta os dois operadores $H$ e $A$, definidos respectivamente pelas equações $(2.1 .1)$ e $(2.1 .13):$

$$
\Lambda=U(H-\beta m) U^{-1}
$$

Esse fato decorre imediatamente da equagāo abaixo:

$$
U \alpha j U^{-1}=\Sigma j \quad, \quad j=1,2
$$

A equivalência unitária da equasăo (2.1.18) deixará o problema de determinagä̌ de condiçóes de contorno de $A$ maito parecido com o problema de determinagão das condicóes de contorno para $H$, como veremos no Capítulo 5. 
É importante também notar que o operador mormento angular total:

$$
J_{z}=\frac{\Sigma_{3}}{2}+t_{z}
$$

onde:

$$
\Sigma_{3}=\left(\begin{array}{cc}
\sigma_{3} & 0 \\
0 & \sigma_{3}
\end{array}\right)
$$

e:

$$
l_{x}=x p_{y}-y p_{x}
$$

comuta formalmente com os operadores $H$ e $\mathrm{A}$, isto é:

$$
\left[H, J_{z}\right]=\left[\Lambda_{1} J_{z}\right]=0
$$

No Apêndice $B$ estââ demonstradas as relaçöes de comutaçăo:

$$
\left[H, l_{z}\right]=-i\left(\vec{\alpha} \wedge \vec{p}+\frac{e \phi}{e r^{2}} \vec{\alpha} \cdot \vec{r}\right)
$$

e:

$$
\left[H, S_{3}\right]=t\left(\vec{\alpha} \wedge \vec{p}+\frac{e}{c} \frac{\phi}{r^{2}} \vec{\alpha} \cdot \vec{F}\right)
$$

que estäo de acordo com a equação (2.1.23) para a harmitoniana. No Apéndice $C$ estāo demonstradas as relaçôes de comutax̧ão:

$$
\left[\Lambda, l_{x}\right]=-i\left(\vec{\Sigma} \wedge \vec{p}+\frac{e \phi}{c} \overrightarrow{r^{2}} \vec{\Sigma} \cdot \vec{r}\right)
$$

e:

$$
\left[\Lambda, S_{3}\right]=i\left(\vec{\Sigma} \wedge \vec{p}+\frac{e}{c} \frac{\phi}{r^{2}} \vec{\Sigma} \cdot \vec{r}\right)
$$

que produzem o resultado da equação (2.1.23) para a helicidade.

$O$ problema das extensors auto-adjuntas para o operador $H$ foi pela primeira vez considerado por Sousa Gerbert ${ }^{[3]}$ e posteriormente por F. A. Coutinho e J. Femando Perez ${ }^{[1,2]}$. Esses autores encontraram uma família de extensöes antoadjuntas a um parámetro (Sousa Gerbert ${ }^{[3]}$ ) e a dois parâmetros (F. A. Coutinlo e J. Fernando Peres ${ }^{(1)}$ ) para o hamiltoniana com a restrição de que este operador 
continuasse possivel de ser decomposto em dois operadores desacoplados, atuando em espinores de duas componentes. Assim há uma indeterminaçäo na escolha das condiçös de contomo, sendo possíveis várias dinàmicas.

No entanto, para comecarmos a entender esse problema, precisamos antes de mais nada, escrevermos a exuaçāo rudial de auto-valores para $H$ e $\Lambda$. Na Seçäo 2.3. zatudaremos o caso do operador $H$ e na Seçấ 2.4 o caso do operador $A$.

\section{2,2. Determinacão das auto-funçôes mais gerais para o operador Hamil toniana.}

Esturnos interessados, agoray em resolver o problema de anto-valores para o operador harniltoniana, isto é:

$$
H \psi_{ \pm}= \pm|E| \psi_{ \pm}
$$

Usando a equaçöo (2.1.1) e as definico das equaços (2.1.2) a (2.1.6), a equaçäo acima pode ser escrita como:

$$
H=\left(\begin{array}{cc}
\vec{\pi} \cdot \vec{\sigma}+\sigma_{3} m & 0 \\
0 & \vec{\pi} \cdot \vec{\sigma}-\sigma_{3} m
\end{array}\right) \psi_{ \pm}= \pm|E| \psi_{ \pm}
$$

A fin de explorarmos a invariância rotacional do hamiltoninna (equagāo (2.2.23)), dada nossa escolha para o potercial vetor $\vec{A}$ da equaça $(2.1 .8)$, vamos utilizar as equaçōes de transformaçäo para coordenadas polares:

$$
\begin{aligned}
& x=r \cos \theta \\
& y=r \sin \theta
\end{aligned}
$$

que implicarn:

$$
\begin{aligned}
& \partial_{x}=\cos \theta \partial_{r}-\frac{\sin \theta}{r} \partial_{\theta} \\
& \partial_{y}=\sin \theta \partial_{4}+\frac{\cos \theta}{r} \partial_{\theta} .
\end{aligned}
$$


Assim podemos escrever a equasăo (2.2.2) como sendo:

$$
H \psi_{ \pm}=\left(\begin{array}{cc}
H+a_{3} m & 0 \\
0 & H-a_{3} m
\end{array}\right) \psi_{ \pm}
$$

onde:

$$
M=\left(\begin{array}{cc}
0 & -i e^{-i t}\left(\partial_{r}-\frac{i}{r} \partial_{\theta}\right) \\
-i e^{i \theta}\left(\partial_{r}+\frac{i}{r} \partial_{\theta}\right) & 0
\end{array}\right)
$$

Podemos tentar uma separaçăo de variáveis do tipo:

$$
\psi_{ \pm}(r)=\left(\begin{array}{cc}
\psi_{ \pm}^{1}(r) & e^{i n \theta} \\
\psi_{ \pm}^{2}(r) & e^{i x^{2} \theta} \\
\psi_{ \pm}^{3}(r) & e^{i n \theta} \\
\psi_{ \pm}^{t}(r) & e^{i n^{2} \theta}
\end{array}\right)
$$

Substituindo a equaça (2.2.9) na equaçăo (2.2.7), conclumos que:

$$
n^{\prime}=n+1
$$

Sendo assim, podemos pensar em duas possibilidades para as auto-funçoes da equaçâo (2.2.9):

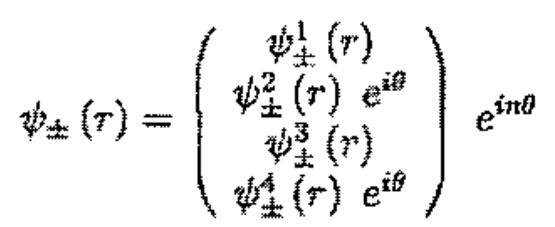

ou:

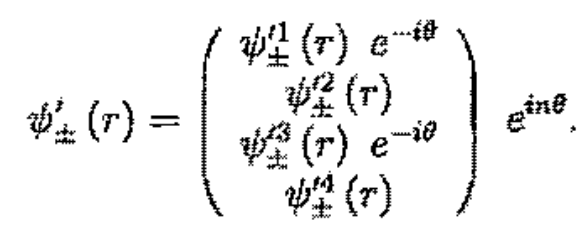

A funço de onda da equaça (2.2.11) acima tem momento angular total igual a $n+\frac{1}{2}$ ( $n$ é o momento angular orbital) como pode ser facilmente checado se usarmos coordenadas polares para escrevermos o operador $J_{z}$ dado pela equacäo (2.1.20) como sendio:

$$
I_{z}=\frac{\Sigma_{z}}{2}-i \partial_{i}
$$


A funçäo de onda da equaçâo (2.1.12) tem momento angular total igual a $n-\frac{1}{2}$, onde $n$ é o momento angular orbital. É claro que podemos obter o mesmo valor para o momento angular total da equaçäo (2.2.12) fazzendo $n \rightarrow n-1$ na equação (2.2.11).

Usando a separação de variáveis da equação (2.2.11), sem perda de generalídade, obtemos a seguinte equação radial de auto-valores de $H$ :

$$
H(r) \psi_{ \pm}(r)=\left(\begin{array}{cc}
h(r)+\sigma_{3} m & 0 \\
0 & h(r)-\sigma_{3} m
\end{array}\right) \psi_{ \pm}(r)= \pm|E| \psi_{ \pm}(r)
$$

onde:

$$
h(r)=\left(\begin{array}{cc}
0 & -i\left(\partial_{F}+\frac{v+1}{r}\right) \\
-i\left(\partial_{r}-\frac{\nu}{r}\right) & 0
\end{array}\right),
$$

onde introduzimos a definiçào:

$$
\nu=n+\phi
$$

Vamos definir:

$$
\psi_{ \pm}(r)=\left(\begin{array}{c}
\alpha \phi_{ \pm \pm}^{1}(r) \\
\beta \phi_{ \pm}^{2}(r)
\end{array}\right)
$$

onde $\phi_{1}^{12}(r)$ são espinore de duas componentes e $\alpha$ e $\beta$ säo duas constantes que regularn as proporços das componentes superior e inferior. Assira, o problema de anto-funçöes da equaçäo $(2.2 .14)$ fica equivalente a resolver o segunte problema de auto-funcöes:

$$
\left(h(r)+3 c_{3} m\right) \phi_{ \pm}^{j}(r)= \pm|E| \phi_{ \pm}^{j}(r),
$$

onde para $s=+1$, estaremos resolvendo o problema de determinação de $\phi_{1}^{1} \mathrm{e}$ pără $s=-1$, o problema de determinação de $\phi^{2}$.

Vamos escrever:

$$
\phi_{ \pm}^{j}(r)=\left(\begin{array}{c}
u_{ \pm}^{j}(r) \\
v_{ \pm}^{j}(r)
\end{array}\right), j=1,2
$$


Daqui em diante, nos referiremos ao operador $H(r)$ da equação (2.2.14) denotandoo simplesmente por $H$. Substituindo as equaçöex $(2.2 .15)$ e (2.2.19) na equaçăo (2.2.18), temos:

$$
\left(\begin{array}{cc}
m s & -i\left(\partial_{r}+\frac{\nu+1}{r}\right) \\
-i\left(\partial_{+}-\frac{v}{r}\right) & -m s
\end{array}\right)\left(\begin{array}{l}
u_{+}^{j} \\
v_{ \pm}^{j}
\end{array}\right)= \pm|E|\left(\begin{array}{l}
u_{ \pm}^{j} \\
u_{ \pm}^{j}
\end{array}\right),
$$

de onde vem que:

$$
u_{ \pm}^{j}=\frac{-i}{ \pm|E|-m s}\left(\partial_{r}+\frac{v+1}{r}\right) v_{ \pm}^{j}
$$

e:

$$
v_{ \pm}^{j}=\frac{-i}{ \pm|E|+m s}\left(a_{r}-\frac{p}{r}\right) u_{ \pm}^{j} .
$$

Substituindo a equaçăo (2.2.22) na equação (2.2.21) temos:

$$
\left(\partial_{k r}^{2}+\frac{a_{k r}}{k r}+\left(1-\frac{v^{2}}{(k r)^{2}}\right)\right) r_{t:}^{i}=0
$$

onde introduzimos a definição:

$$
k=\sqrt{E^{2}-m^{2}} .
$$

A solnção da exuação (2.2.23) é do tipo:

$$
u_{ \pm}^{j}(k r)=J_{\mu \nu}(k r)+c_{ \pm} J_{-\nu}(k r) .
$$

Lembrando qute a componente inferior $\left(y_{ \pm}\right)$está conectada corm a superior $\left(u_{ \pm}\right.$) através da equagão (2.2.22) (fato crucial na solução do problema) e usando as fórmulas de recorrência da referência [10]:

$$
\begin{gathered}
\frac{d}{d x} J_{\nu}(x)=J_{y-1}(x)-\frac{\nu}{x} J_{\nu}(x) \\
\frac{d}{d x} J_{\nu}(x)=-J_{v+1}(x)+\frac{\nu}{x} J_{\nu}(x),
\end{gathered}
$$


temos que:

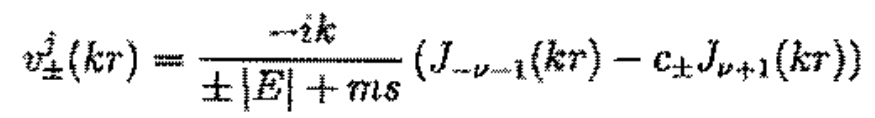

Os resultados da equaçös $(2.2 .25)$ e (2.2.27) permitern escrever as auto-funçōes da equagão $(2.2 .19)$ como sendo:

$$
\phi_{ \pm}^{1}(k r)=\left(\begin{array}{c}
J_{-\nu}(k r)+c_{ \pm} J_{\nu}(k r) \\
\frac{-k_{k}}{ \pm|E|+m}\left(J_{-t s-1}(k r)-c_{ \pm} J_{\nu+1}(k r)\right)
\end{array}\right)
$$

e:

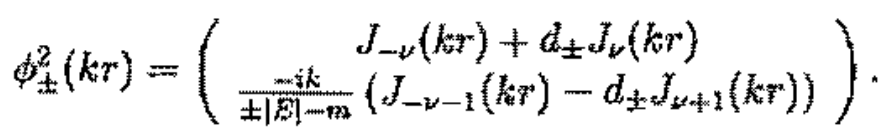

De modo que as auto-fungöes (de quatro componentes) de $H$ da equaça $(2.2 .14)$ são;

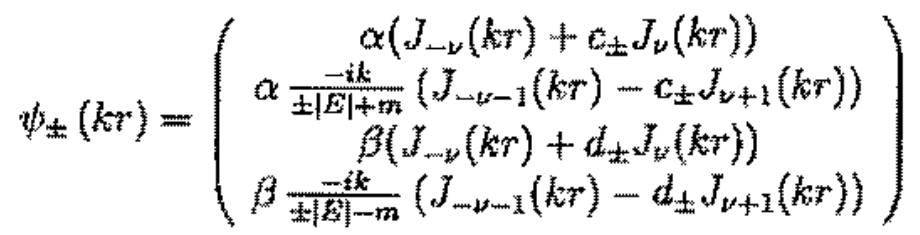

Considerando a forma das solugös para as antofunçōes de $H$ da equaça acima e lembrando do comportamento na origem das funcōes de Bessel (10):

$$
y_{\nu}(x) \sim \frac{1}{\Gamma(1+\nu)}\left(\frac{x}{2}\right)^{\nu}
$$

vemos que para $\nu=n+\phi \geq 0$ e para $v \leq-1$, apenas as soluyoues regulares na origem säo admissiveis, pois nexses casos as soluçōes singulares na origem säo nāo normalizáveis na vizinhanga da origem.

Entretanto, para o caso $-1<v<0$, é impossivel impor a regularidade na orizem simultaneamente para todas as componentes da funçäo, havendo assim uma indeterminaçäo na escolha das condigôes de contorno (note que isso acontece devido a relaçâ da equaça (2.2.21) entre as componentes superior e inferior do espinor de auto-funçoes de $H$ ). Nesse caso a soluçöo singular na origem é normalizável na vizinhança da origem. Sendo assim possiveis extensöes autoadjuntas para o hamiltoniana no caso em que $-1<\nu<0[1,2,3]$. 
Para todo valor nâo inteiro do fluxo $\phi$, ha exatamente um valor de $n$ (que corresponde a uma onda parcial):

$$
n=-[\phi]-1
$$

onde $[\phi]$ representa o maior inteiro menor ou igual $\phi$, tal que $\nu=n+\phi$ está no intervalo crítico $]-1,0[$.

2.3. Determinação das auto-funções mais gerais para o operador Helicidade.

Estamos interessados agora em resolver o problema de auto-valores para o operador helicidade, isto é:

$$
A \psi_{ \pm}= \pm|\lambda| \psi_{ \pm}
$$

Usando a equaçäo (2.1.13) da definiçăo formal do operador $\Lambda$ e lembrando da equivalềncia unítíria da equaçäo (2.1.18), podemos escrever o operador $\Lambda \mathrm{em}$ coordenadas polares como sendo dado por:

$$
\Lambda=\left(\begin{array}{cc}
0 & \mathcal{H} \\
\mathcal{H} & 0
\end{array}\right)
$$

onde $\mathcal{H}$ é dado como na equação (2.2.8) da seçäo anterior. Usando a mesma separação de yariáveis đa equação $(2.2 .11)$, obtemos a segunte equação radial de auto-funçôes para $\Lambda$ :

$$
\Lambda(r) \psi_{ \pm}(r)=\left[\begin{array}{cc}
0 & h(r) \\
h(r) & 0
\end{array}\right] \psi_{ \pm}(r)= \pm|\lambda| \psi_{ \pm}(r)
$$

onde $h(r)$ está definido na equaçäo (2.2.15). Daqui em diønte, frequentemente nos referiremos ao operador $\Lambda(r)$ denotando-o por $\Lambda$.

Para encontrarmos as auto-finçöes de $\Lambda$ vamos escrever os espinores $\psi_{ \pm}(r)$ de quatro componentes que representam as auto-funçōes a serem encontradas como sendo:

$$
\psi_{ \pm}(r)=\left(\begin{array}{l}
\psi_{ \pm}^{(1)}(r) \\
\psi_{ \pm}^{(2)}(r)
\end{array}\right)
$$


de modo que a equaxäo (2.3.3) implique nas seguintes relacoto entre as componentes superior wis e inferior $y_{i}^{2}$ :

$$
\begin{aligned}
& h(r) \psi_{ \pm}^{(2)}(r)= \pm|\lambda| \psi_{ \pm}^{(1)}(r) \\
& h(r) \psi_{ \pm}^{(1)}(r)= \pm|\lambda| \psi_{w}^{(2)}(r)
\end{aligned}
$$

Substituindo a equaço $(2.3 .6)$ na equaça $(2.3 .5)$ on vice-versa, obtemos que:

$$
h^{2}(r) \psi_{ \pm}^{i}(t)=\lambda^{2} \psi_{ \pm}^{j}(t) \quad j=1,2
$$

Assim, para resolvermos o problema de auto-funçōes da eguação (2.3.s) vamos antes resolver o problema de auto-funçós de duas componente da equação (2.3.7) acinne.

Usando o resultado da equaçã̃o (2.2.15) podemos escrever o operador $h^{2}$ como sendo o seguinte operador diagonal:

$$
h^{2}(r)=\left(\begin{array}{cc}
\partial_{r}^{2}+\frac{1}{r} \partial_{r}-\frac{z^{2}}{r^{2}} & 0 \\
0 & \partial_{r}^{2}+\frac{1}{y_{r}}+\frac{(\nu+1)^{2}}{r^{2}}
\end{array}\right)
$$

onde $v=n+6$

De modo que, escrevendo as funçoes de onda da equagăo (2.3.7) sob a forma:

$$
\psi_{ \pm}^{j}(\tau)=\left(\begin{array}{c}
u_{u}^{j}(r) \\
v_{s}^{j}(r)
\end{array}\right), j=1,2
$$

e usando o resultado da equagäo (2.3.8) acima, temos:

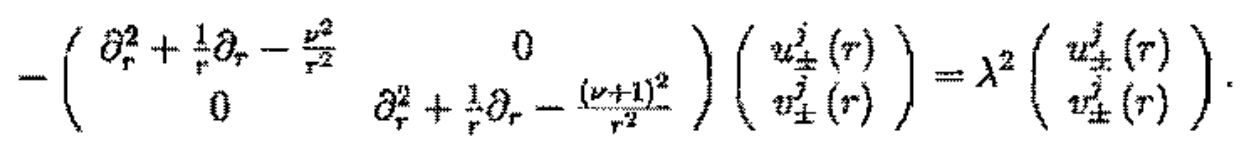

Da equação acima surgem as equaçoes desscopladas para $u_{2}^{j}$ e $v_{ \pm}^{i}(r)$ :

$$
\left(\partial_{r}^{2}+\frac{1}{r} \partial_{r}-\frac{\nu^{2}}{r^{2}}\right) u_{ \pm}^{j}(r)=-\lambda^{2} u_{+}^{j}(r)
$$

e:

$$
\left(\partial_{r}^{2}+\frac{1}{r} \partial_{r}-\frac{(y+1)^{2}}{r^{2}}\right) v_{ \pm}^{2}(r)=-\lambda^{2} v_{ \pm}^{j}(r)
$$


cujas soluçöes mais gerais säo do tipo:

$$
u_{ \pm}^{j}(\lambda r)=J_{m \nu}(\lambda r)+a_{ \pm} J_{\nu}(\lambda r)
$$

e:

$$
v_{ \pm}^{\hat{H}}(\lambda r)=J_{w-1}(\lambda r)+b_{ \pm} J_{p+1}(\lambda r)
$$

Assim os espinores $\psi_{ \pm}^{3}$ (anto-funçós do operador $h^{2}(r)$ ) têtn a forma:

$$
\psi_{ \pm}^{j}(\lambda r)=\left(\begin{array}{c}
J_{-D}(\lambda r)+a_{ \pm} J_{\nu}(\lambda r) \\
J_{-t-1}(\lambda r)+b_{ \pm} J_{\nu+1}(\lambda r)
\end{array}\right) j=1,2 .
$$

Para encontrarmos, agora, as antom-fingöes do operador $\Lambda$, devemos impor que os espinores da equação (2.3.15) obedecam as equaçōes $(2.3 .5)$ e (2.3.6) que relacionam as componentes superior $\psi_{ \pm}^{(1)}$ e inferior $\psi_{ \pm}^{(2)}$ do espinor de quatro componentes de auto-funcōes de $\Lambda$.

Assim tomando $\psi_{ \pm}^{(n)}(\lambda r)$ como sendo dado pela equacäo $(2.3,15)$, as equaçocs $(2.3 .5)$ e $(2.3 .6)$ tornecm:

$$
\psi_{ \pm}^{(2)}(\lambda r)=\left(\begin{array}{c}
\mp i\left(b_{*} J_{v}(\lambda r)-J_{-\nu}(\lambda r)\right) \\
\mp i\left(J_{-\nu-1}(\lambda r)-a_{ \pm} J_{v+1}(\lambda r)\right)
\end{array}\right)
$$

A expresâo da equaça (2.3.16) acima foi cnoontrada usando os resultados das equaģoes (2.2.26) e (2.2.27). Assim, as auto-funçöes de $\Lambda$ quando tomarnos ${ }_{4}^{(1)}$ como na equaça (2.3.15) e $\psi_{ \pm}^{(2)}$ como na eqquaça (2.3.16) poden ser escritas como sendo:

$$
\psi_{ \pm|\lambda|}(\lambda r)=\left(\begin{array}{c}
J_{-\nu}(\lambda r)+a_{ \pm} J_{\nu}(\lambda r) \\
J_{-\nu-1}(\lambda r)-b_{ \pm} J_{\nu+1}(\lambda r) \\
\mp i\left(b_{ \pm} J_{v}(\lambda r)-J_{-v}(\lambda r)\right) \\
\mp i\left(J_{-b-1}(\lambda r)-a_{ \pm} J_{\nu+1}(\lambda r)\right)
\end{array}\right)
$$


Trocando $\psi_{ \pm}^{(1)}$ por $\psi_{ \pm}^{(2)}$ na equacăo (2.3.17) (ou seja, tomando $\psi_{ \pm}^{(2)}$ como sendo dado por (2.3.15)), respeitando as condiços das equaços (2.3.5) e (2.3.6) (que garantem que as funções obtidas são auto-funçöes do operador $\Lambda$ ), temos as seguintes auto-funçóes de $\Lambda$ :

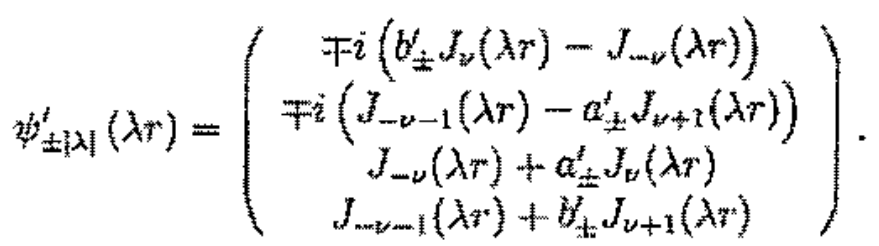

Assim existem quatro alıto-funçös do operador $\Lambda$ : duas com auto-valor positivo $+|\lambda|$ e duas com auto-valor negativo $-[\lambda \mid$, dadas pelas equaçóes (2.3.17) e (2.3.18) acima.

Analisando a forma das soluçöes para as auto-funcöes de $\Lambda$ das equacões scima e lembrando do cornportamento na origern das funçōes de Bessel dada pela equaç̆o (2.2.32) vemos que para $\nu=n+\phi \geq 0$ e para $\nu \leq-1$, apenas as soluçöes regulares na origern săo admissíveis, pois nesses casos, as componentes singulares na origem săo näo localmente normalizáveis na vizinhança da origem.

No entanto, para o caso $-1<v<0$, é impossivel impor a cordiçäo de regun laridade simultaneamente para todas as componentes, havendo assim uma indeterminaça na escolha das condiçöes de contorno (note que isso acontece devido às relaçöes das equaçöes (2.3.5) e (2.3.6) entre as componentes superior $\psi_{ \pm}^{(1)}(r)$ e inferior $\psi_{ \pm}^{2 y}(r)$ do espinor de anto-funçöes de $\Lambda$ ). As soluçóes singulares na origem são normalizáveis na vizinhança da origem no caso ern que $v:]-1,0$. $\mathrm{Sendo}_{\mathrm{i}}$ assim possiveis extensóes anto-adjuntas para a helicidade (ou seja, podemos tomar differentes auto-funcöes que correspondem a diferentes proporçöes entre as soluçôes regular e singular).

Isso é o mesmo que ocorre para o operador hamiltoniana, descrito na seçäo anterior. Da mesma forma que naquele caso, teremos que, para todo valor não

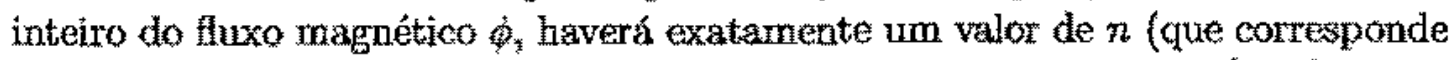
a uma onda parcial) $n=-[\phi]-1$, tal que $\nu$ está no intervalo crítico $]-1,0[$. Nesse intervalo os operalores $H$ e $A$ não estäo bem definidos, havendo a necessidade de especificasão das condições de contorno na origem a serem satisfeitas pelas auto-finçōes de $H$ e de $\Lambda$, o que é equivalente ao problema de determinação das extensōes auto-adjuntas para esses operadores ${ }^{\{1,2,3)}$.

Nesse trabailho, estamos interessados em definir os operadores $H$ e $A$ em 
dominios (comuns a ambos) tais que ales sejam automadiuntos e que a belicidade seja uma quantidade conservada.

Lembremo-nos de que esse problema nāo possui soluçāo trivial imediata, uma vez que, conforme dissemos anteriormente na Seçäo 2.1 , apenas a comutaçăo formal entre os operadores $H$ e $\Lambda$ não garante a conservação da helicidade e conse. quentemente não implica na obtençăo de auto-funçöx simultâneas (para $H$ e $\Lambda$ ) para toda dinḱmica definida por $H$ atravk de algum conjunto de condiçöes de contorno.

Nos próximos capitulos procederemos a extensões auto-adjuntas para os operadores $H$ e $A$ e investigaremos a possibilidade de obtenção de auto-funçós comums para exsas extensōes.

No entanto, antes de considerarmos o problema das extensões auto-adjuntas para $H$ e $\Lambda$ no caso da particula de Dirac em duas dimensões sujeita a uma linha de fluxo magnético na origem, vamos considerar o caso mais simples de uma partícula live $(\phi=0)$ em duss dimensōes. Na próxima seço vamos investigar a possibilidade de conservagäo da helicidade para esse cano mais simples.

\section{4. $O$ Caso Livre.}

Consideraremos nessa seçäo o problema de una partícula de Dirac livre em duas dimensöts. Nesse caso o operador hamiltoniana é essencialmente auto-adjunto e suas auto-funçōes säo sempre regulare na origem.

Inicialmente vamos encontrar quais säo escas auto-funçōes mais gerais e a seguir verificar, a partir dessus auto-funçoes encontradas, que é possivel obter auto-funçōes simultâneas para os operadores hamiltoniana e helicidade.

Assim, vamos agora considerar a equaçäo radial de auto-fumçöes de $H$, que pode ser obtida fazendo-se $\phi=0$ na autaça $(2.2 .14)$

$$
H(r) \psi(r)= \pm|E| \psi \mid t(r)
$$

onde:

$$
H(r)=\left(\begin{array}{cc}
h(r)+\sigma_{3} m & 0 \\
0 & h(r)-\sigma_{3} m
\end{array}\right)
$$

$\operatorname{com} *$

$$
h(r)=\left(\begin{array}{cc}
0 & -i\left(\partial_{r}+\frac{l+1}{r}\right) \\
-i\left(\partial_{r}-\frac{1}{r}\right) & 0
\end{array}\right)
$$


onde $l$ te numero inteiro azsociado a onda parcial " $l$ ".

Varnos escrever as autofunçöes $\psi_{ \pm}(r)$ sob a forma:

$$
\psi_{ \pm}=\left(\begin{array}{cc}
\alpha & \phi_{ \pm}^{(1)} \\
\beta & \phi_{ \pm}^{(2)}
\end{array}\right)
$$

com $\phi_{ \pm}^{(1), 2)}$ Eendo espinores de duas componentes e $\alpha$ e $\beta$ sendo duas constantes para um carto valor fixo da energia. Assim, o problema de determinaçäo de auto funçôes da equação $(2,4.1)$ fica equivalente ao seguinte problema de determinação de anto-fumçöes de duas componentw:

$$
\left(h+\sigma_{3} m g\right) \phi_{ \pm}^{3}= \pm|m| H_{1}^{3}, \quad j=1,2
$$

onde para $s=+1$, estarernos resolvendo o problema de determinacăo de $\phi_{ \pm}^{(1)}$ e para $s=-1$, estaremos resolvendo o problema de determinaçăo de $\phi_{ \pm}^{(2)}$.

Definindo:

$$
\phi_{ \pm}^{j}=\left(\begin{array}{c}
\frac{u_{i}^{j}}{j} \\
v^{j}
\end{array}\right)
$$

e usando a equaçäo $(2.4 .3)$, a equaçäo (2.4.5) se torna:

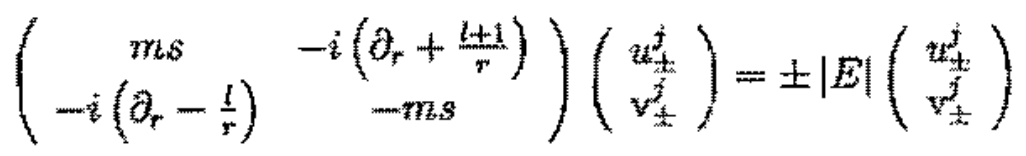

Da equaçă丶 $(2.4 .7)$ acima vem que:

$$
\begin{aligned}
& m s u_{ \pm}^{i}-i\left(\partial_{r}+\frac{l+1}{r}\right) v_{ \pm}^{j}= \pm|E| u_{\text {s }}^{j} \\
& -i\left(\partial_{r}-\frac{1}{r}\right) u_{ \pm}^{i}-m s v_{ \pm}^{j}= \pm|E| v_{t}^{j}
\end{aligned}
$$

de onde tiramos:

$$
u_{ \pm}^{j}=\frac{-i}{ \pm|E|-m s}\left(\partial_{r}+\frac{l+1}{r}\right) v_{ \pm}^{j}
$$

e:

$$
v_{=}^{i}=\frac{-i}{ \pm|E|+m s}\left(\partial_{r}-\frac{l}{r}\right) u_{x}^{i}
$$


Substituindo a equaça (2.4.11) na equacão (2.4.10), obtemos a seguinte equagão para $u_{j}^{j}(r)$ :

$$
\left(\partial_{k r}^{2}+\frac{1}{r} \partial_{k r}+\left(1-\frac{l^{2}}{(k r)^{2}}\right)\right) u_{ \pm}^{*}= \pm|E| u_{ \pm}^{j}
$$

onde introduzimos a definicào:

$$
k=\sqrt{E^{2}-m^{2}}
$$

A soluça mais geral da equacäo $(2.4 .12)$ para $l$ inteiro $\hat{\text { ê }}$

$$
u_{i}^{2}=J_{l}(k r)
$$

Lenbremo-nos que $J_{-l}=(-)^{4} J_{l}$. Substituindo o resultado da equaçäo (2.4.14) acima na equaçäo $(2,4.11)$, a qual relaciona as componentes inferior $\left(v^{j}\right)$ e superior $\left(u_{\text {出}}^{j}\right)$, temos:

$$
v_{\text {s. }}^{i}=\frac{i k}{ \pm|E|+m s} u_{i+1}(k r)
$$

onde usamos o resultado da equaçäo $(2,2.26)$.

Substititindo os resultados das equacöes $(2.4 .14)$ e (2.4.15) na equaçäo $(2.4 .6)$ encontramos para $s=+1$ a funçäo $\phi_{ \pm}^{(1)}$ e para $s=-1$ a funçâo $\phi_{ \pm}^{(2)}$ como sendo iguais a:

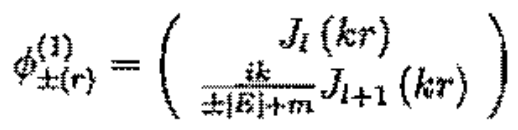

e:

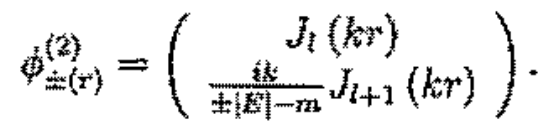

De modo que a equaçâo $(2.4 .4)$ para as auto-fuçõos mais gerais de quatro componentes do operador $H(r)$, pode ser escrita como:

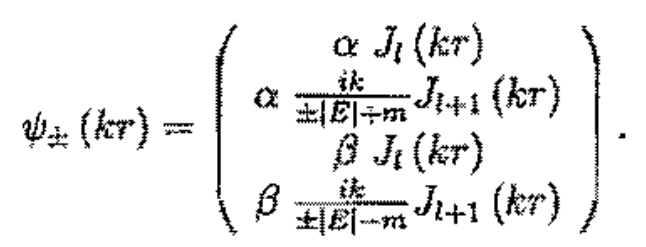


Vejamos agora se conseguimos encontrar anto-funçōes simultâneas para os operadores $H$ e $A$ para algum valor dos coeficientes $\alpha$ e $\beta$ que regulam respectivamente as proporçôes das componentes superior e inferior. Para isso vamos impor que:

$$
A \psi_{4}=\lambda \psi_{ \pm}
$$

Lembrando (veja equação (2.3.3)) que $\Lambda$ pode ser escrito como:

$$
\Lambda=\left(\begin{array}{ll}
0 & h \\
h & 0
\end{array}\right)
$$

e usando a equaçăo (2.4.4), a equação (2.4.19) acima pode ser escrita como:

$$
\left(\begin{array}{ll}
0 & h \\
h & 0
\end{array}\right)\left(\begin{array}{ll}
\alpha & \phi_{ \pm}^{(1)} \\
\beta & \phi_{ \pm}^{(2)}
\end{array}\right)=\lambda\left(\begin{array}{cc}
\alpha & \phi_{ \pm}^{(1)} \\
\beta & \phi_{ \pm}^{(2)}
\end{array}\right),
$$

de onde vem que:

$$
\begin{gathered}
\beta h \phi_{ \pm}^{(2)}=\lambda \alpha \phi_{ \pm}^{(1)} \\
\alpha h \phi_{ \pm}^{(1)}=\lambda \beta \phi_{ \pm}^{(2)} .
\end{gathered}
$$

Devido à equaçäo (2.4.5), as eqquaçöes (2.4.21) e (2.4.22) acina podem ser escritas como sendo:

$$
\begin{aligned}
& \beta\left( \pm|E|+\sigma_{3} m\right) \phi_{ \pm}^{(2)}=\lambda \alpha \phi_{ \pm}^{(1)} \\
& \alpha\left( \pm|E|-\sigma_{3} m\right) \phi_{ \pm}^{(1)}=\lambda \beta \phi_{ \pm}^{(2)}
\end{aligned}
$$

As equaçōes acima fornecem o sisterna:

$$
\begin{aligned}
& \beta( \pm|E|+m)=\lambda \alpha \\
& \alpha( \pm|E|-m)=\lambda \beta
\end{aligned}
$$

A. condiçāo de existência de soluçōes $(\alpha$ e $\beta)$ para o sistema acima fornece:

$$
\lambda= \pm \sqrt{E^{2}-m^{2}}= \pm k
$$


que são os auto-valor da helicidade.

Para $\lambda=+k$, temos a seguinte relaça entre os coeficientes $\alpha$ e $\beta$ :

$$
\beta=\frac{k}{ \pm|E|+m} \alpha
$$

e para $\lambda=-k_{y}$ temos:

$$
\beta=\frac{-k}{ \pm|E| \div m} \alpha
$$

Assim, tomando $\alpha$ e $\beta$ como nas equaçoes $(2.428)$ e $(2.4 .29)$, obtemos as anto-fungöes simultâneas de $H$ e $\Lambda$ como sendo as seguintes:

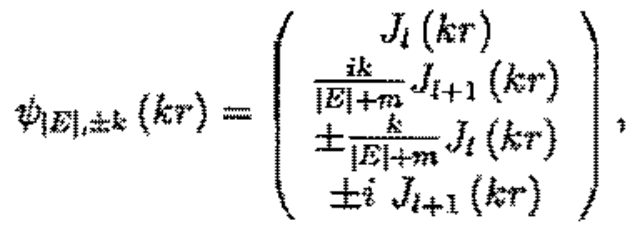

$$
\begin{aligned}
& \psi_{-|\mathrm{j}|, \pm k}(k r)=\left(\begin{array}{c}
J_{l}(k r) \\
\frac{-i k}{|E|-m} J_{l+1}(k r) \\
\mp \frac{k}{|E|-r r} J_{l}(k r) \\
\pm i J_{1+1}(k r)
\end{array}\right) .
\end{aligned}
$$

Vemos, então, que para o caso livre $(\phi=0)$, como os operadores $H$ e $A$ são essencialmente auto-adjuntos, o problema de daterminação das auto-funçôs simul tâneas é facilmente resolvido. Para o caso nă livre, quando existe a presença da linha de fluxo magnético na origem, como verenos nos próximos capítulos, além das condições que dăo as proporçöes entre as componentes superiores e inferiores dos espinores surgem outras condições que relacionam os parâmetros das extensöes e que tomarn o problema mais complicado. 


\section{Capítulo 3 \\ Um Método Heurístico para verificação da necessidade de extensōes auto-adjuntas.}

No capítulo anterior verificamos, através da análise das autonfuncöes dos operadores hamiltoniana (dada pela equaçäo (2.2.31)) e das auto-funçós do operador helicidade (dadas pelas equaços $(2.3 .17)$ e $(2.3 .18)$ ), que năo são possiveis soluçöes singulares para $\nu=n+\phi \geq 0$ e $\nu \leq-1$, uma vez que essas soluçoes näo säo integraiveis na vizinhança da origem nesses casos. Para $-1<y<0$, verificamos que as soluçöes singulares sâo localmente normalizakeis na vizinhanģa da origem, sendo impossivel, nesse caso, impor a regularidade na origem simultaneamente para as componentes superior e inferior do espinor de auto-funçōes de $H$ (e de $\Lambda$ ). Assim concluímos que para $-1<\nu<0$ são possíveis extensōes auto-adjuntas para $H$ e para $\Lambda$ que podern ser obtidas pelo método dos índices de deficiència, que apresentaremos no Capítulo 4.

No entanto, $e$ bastante instrutivo notar que podemos chegar a essa mesma conclusâo, quanto à possibilidade de extensōes auto adjuntas, sem precisar obter as auto-funçöes de $H$ e de $\Lambda$, para só então analisar os intervalos de $\nu$ para os quats as soluçôes singulares na origem säo admissiveis.

Podemos obter o intervalo de $\nu$ para o qual säo possíveis extensōes autoadjuntas para $H$ e $A$ atraves de $1 \mathrm{~m}$ método heuristico bastante simples que consiste em se fazer a andise do comportomento da exunçio diferencial pore as componentes superior e inferior to espinar de atoto-funços no vizinhança da orgigem,

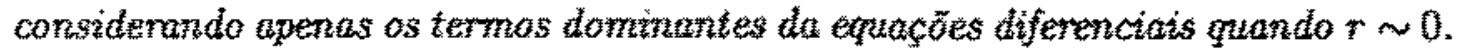

A oquasão diferencial para a componente superior do espinor de auto-funcôtes de $H$, conforme mostra a equaço (2.2.23) tem a forma:

$$
\frac{d^{2} u}{d r^{2}}+\frac{1}{r} \frac{d u}{d r}-\left(\frac{t^{2}}{r^{2}}-k^{2}\right) u=0
$$

A equação diferencial para a componente superior do espinor de auto-funçôes de $\Lambda_{1}$ conforme a equaçāo $(2.3 .11)$, é da forma:

$$
\frac{d^{2} u}{d r^{2}}+\frac{1}{r} \frac{d u}{d r}-\left(\frac{\nu^{2}}{r^{2}}-\lambda^{2}\right) u=0
$$


Considerando apenas os termos dominantes nas duas diferenciais acima quando $r \sim 0$ temos a seguinte equaçăo diferencial para as componentes superior das autofunçöes tanto de $H$ quanto de $A$ :

$$
\frac{d^{2} u}{d r^{2}}+\frac{1}{r} \frac{d u}{d r}-\frac{\nu^{2}}{r^{2}} u=0
$$

Tentando uma solugão particular para a equação (3.3) acima, da forma:

$$
u(r)=c r^{\alpha},
$$

onde $\alpha$ e uma constante a ser determinada, concluímos que:

$$
\alpha= \pm \nu
$$

Esse resultado mostra que deve haver doís tipos de soluçăo para as equaçoes diferenciais (3.1) e (3.2) com comportamento na origem distintos.

Existe uma solıção regular, que na origem se comporta como $r^{\nu}$ para $\nu>0$ on $\operatorname{con} r^{-\alpha}$ se $\nu<0$. Existe também uma solução singular na origem, que se comporta (na vizinhança da origem) como $r^{-\nu}$ para $\nu>0$ ou com $r^{\prime \prime}$ para $\nu<0$.

Varıos analisar agora as equaçöes diferenciais para as componentes inferiores dos espinores de auto-fureçốes de $H$ e de $A$.

Note que as equaçōes (2.2.4) e (2.2.22) mostram que a equaçăo diferencial para as componentes inferiores da auto-funçoes de $H$ tem a forma:

$$
\frac{d^{2} v}{d r^{2}}+\frac{1}{r} \frac{d v}{d r}-\left(\frac{(v+1)^{2}}{r^{2}}-k^{2}\right) v=0
$$

Do mesmo modo a equasâa diferencial para a componente inferior do espinor de auto-funç̧os de $\Lambda$, conforme a equaçăo $(2.3 .12)$, é:

$$
\frac{d^{2} v}{d r^{2}}+\frac{1}{r} \frac{d v}{d r}-\left(\frac{(y+1)^{2}}{r^{2}}-\lambda^{2}\right) v=0
$$

Considerando novamente apenas os termos dominantes das duas equaçöes diferenciais na vizinhança da origem e tentando uma soluşä̌o do tipo $r^{a}$, concluimos que:

$$
\alpha= \pm(t+1) \text {. }
$$

Esse resultado mostra que as equaçoes (3.6) e (3.7) admitem dois tipos de solução com comportamentos distintos na origem: uma sendo regular e outra singular na origerm. 
Resta saber agora se as soluççes singulares na origem são admissiveis para algurn intervalo de $u$, ou seja, se sầ de quadrado integrável na vizinhança da origem para certos valores de $\psi$

Lembrando que estamos tratando de um problema em duas dimensōes temos que, portanto, soluçôes do tipo $r^{\beta}$ são admissíveis (integráveis na origem) sర se $2 \beta+1>-1$.

Assim a análise da possibilidade de soluções singulares integráveis na origem para as componentes superior e inferior nos leva a conclusäo que, para $y \geq 0 \mathrm{e}$ $\nu \leq-1$, as soluçöes siugulares não sāo adruissíveis. De modo que, para $\nu \geq 0$ e $\nu \leq-1$ os operadores $H$ e $\Lambda$ säo essencialmente auto-adjuntos, näo requerendo nem admitindo outras extensōes (havendo apenas uma única extensâo possivel). Para $-1<y<0$, alérn das soluçốs regulares são possiveís também as soluçöes singulares que săo normalizáveis na origen. De modo que sâo possiveis diferentes extensöes auto-adjuntas (na verdide familas a quatro parâmetros de ettensöes) para os operadores hamiltoniana $H$ a helicidade $\Lambda$ para $-1<\nu<0$, que corresponden, de fato, às uhinias soluçōes possiveis onde são tomadas diferentes proporçōes entre as soluçôes regular e singular. 


\section{Capitulo 4}

\section{Teoria das Extensōes Auto-Adjuntas de von Neumann}

\subsection{Introdução.}

Sabemos que observáveis em Mecânica Quãntica sảo operadores que atuam em espaços de Hitbert. Para os matemáticos, um operador é definido pela sua açāo e por seu domínio. Nós, físicos, em geral ignoramos o domínio, pensando que basta tomar o conjunto de vetores tais que a açầo do operador nẵo tire este vetor do espaço de Hilbert.

Entretanto os operadores admissiveis em Mecânica Quântica devem ser obrigatoriamente auto-adjuntos. Em certcas domínios um operador pode não ser autoadjunto. Torna-se, portanto, imperativo estudar esta questäo com maior cuidado.

Assim, sejam $f$ e $g$ dois elementos de um espaço de Hilbert e $B$ um operador linear. $O$ adjunto de $B, B^{*}$, é definido como:

$$
\langle f \mid B g\rangle=\left\langle B^{*} f \mid g\right\rangle
$$

O operador $B^{*}$ pode existitr on não. Se existir e se $B^{*}$ tiver a mesma ação que $B$, entäo $B$ é dito simétrico. Se o adjunto $B^{*}$ for igual ao operador $B$, isto é, se tiver a mesma açăo e o mesmo domínio, entẩo o operador é dito auto-adjunto.

Surgem, então, as perguntas:

"Como saber se um operudor é auto-adjunto?"

"Como construit operadores auto-adjuntos?"

Tentaremos responder a essas questöes posteriormente na Seçäo 4.3. Na próxíma seção apenas definiremos algumas noçōes nesessárias para o entendimento do assunto. 


\subsection{Algumas definiçōes.}

Vamos, agora, introduzir alyuns conceitos e deiniçoes que seräo usados na próxima seçäo quando discutiremos os critérios para se saber se um operador admite extensōes auto-adjuntas e o mettodo de von Neumamn para obte-les. Se o leitor preferir poderá passar a leitura das próximas sefóes, consultando essa se e quando for necessánio. Consultamos as referências $[11],[12],[13],[14],[23],[24],[25],[26]$ e [27] para a elaboração dessa e das próximas secôtos.

Definiçäo: Dizemos que uma sequência de vetores $\psi_{n}$ é uma sequência de Couchy se:

$$
\left\|\psi_{*}-\psi_{m}\right\| \rightarrow 0, \text { quando } m, n \rightarrow \infty
$$

onde o número positivo $\|y\|=\sqrt{(\psi|\psi| \psi \mid}$ chanado de norma ou comprimento do vetor $\psi$

Toda sequência de vetores $\psi_{n}$, num determinado espaço de Hilbert $H$, que converge para um vetor limite $\psi$, é uma seculência de Cauchy, pois se:

$$
\psi_{n} \rightarrow \psi
$$

isso signitica que:

$$
\left\|\psi_{n}-b\right\| \rightarrow 0 \text {, quando } n \rightarrow \infty
$$

Então:

$\left\|\psi_{m}-\psi_{m}\right\|=\left\|\psi_{n}-\psi+\psi-\psi_{n}\right\| \leq\left\|\psi_{n}-\psi\right\|+\left\|\psi_{m}-\psi\right\| \rightarrow 0$, quando $n, m \rightarrow \infty$

Nessa demontração usamos a desigualdade triangular ${ }^{[11], 112]}$.

Definiçäo: um espaço 6 dito completo, se toda sequência de Cauchy de vetores desee espaço converge.

Definição: um conjunto é dito fechato se toda sequência convergente de vetores $\psi_{n}$ desse conjunto converge para um vetor lirnite $\psi$ que tambem pertence a esse conjunto.

Definição: se um espaço linear com um produto interno definìdo é completo, entăo é chamado de capaco de Hilbert. 
Definição: um espaço de Hilbert é separável se possuir uma base ortonornal que consiste em um conjunto contável (finito ou infinito) de vetores, digamos $\phi_{1}$, $\phi_{2}, \phi_{3}, \ldots, \phi_{j}, \ldots$

Definiçäa: seja $A$ um conjunto de vetores tal que $A \subset M$. Se para todo vetor $\psi \in M$, existe uma sequễncia de vetores $\psi_{n}$ em $A$ que converge para $\psi$, entẫo $A$ ê dito denso em $M$.

Definição: um operador linear $B$ é limitzado se existe um número positivo $C \geq 0$ tal que:

$$
\|B \psi\| \leq C\|\psi\|, \quad \text { para } \forall \psi
$$

Caso contrário o operador é chamado näo-limitado. O menor número $C$ que satisfaz essa propriedade é chamado de norma de $B$ e é denotado por $\|B\|$.

Definiçäo: umo operador linear $B$ é contínuo se para toda sequência de vetores $\psi_{n}$ que converge para um vetor limite $\psi$, acontecer que $B \psi_{n} \rightarrow B \psi$. Um operador é contínuo se e só se for limítado ${ }^{[2]}$, como se pode verificar.

Definiçäo: $O$ domínio de um operador $B$ denotado por $D(B)$ é o conjunto de vetores $\psi$ para os quais $B \psi$ está definido, ou seja, $B \psi$ também é um vetor pertencente ao espaço de Hilbert em questão.

Um operador limitado está definido para todos os vetores do espaco de Hilbert

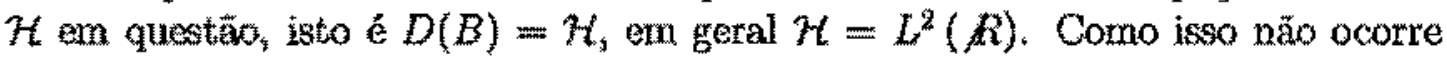
para um operador nâo-limitado, o domínio do operador é um conjunto de vetores 4 tal que $\psi \in D(B) \subset T$.

Definụ̈̂a: $A \times B$ éo produto cartesiano de $A$ e $B$, isto é, o conjunto de pares ordenados:

$$
\{(a, b) \mid a \in A, b \in B\} .
$$

Definiçẵo: $O$ gráfico de urn operador linear $B$ denotado por $\Gamma(B)$ é o conjunto:

$$
\Gamma(B)=\{(\varphi, B \varphi) \mid \varphi \in D(B)\}
$$

Assim o gráfico de $B$ é um subconjunto de $H \times H$ é um eapaxo de Hilbert com o produto interno:

$$
\left\langle\left(\varphi_{1}, \psi_{1}\right) \mid\left(\varphi_{2}, \psi_{2}\right)\right\rangle=\left\langle\varphi_{1} \mid \varphi_{2}\right\rangle+\left\langle\psi_{1} \mid \psi_{2}\right\rangle
$$

Definição: $O$ operador $B$ é dito fechado se $\Gamma(B)$ é um subconjunto fechado de $H \times H$ 
Teorema: Se $B$ é um operador densamente definido, então o adjunto de $B$, $B^{*}$ é único. definicão:

Seja $B: D(B) \subseteq X \rightarrow X$ um operador linear densamente definido. Por

$$
v \in D\left(D^{*}\right)
$$

se existe um vetor $w \in X$, tal que

$$
(v \mid B u)=\langle u \mid u\rangle, \text { para todo } u \in D(B)
$$

e definimos:

$$
B^{*} v=w
$$

Assim, obtemos o opertor adjunto $B^{*}: D\left(B^{*}\right) \subseteq X \rightarrow X$. Para que essa definição faça sentido, $D(B)$ deve ser denso em $X$. De fato, suponha que a relagäo (4.2.9) seja verdadeira se trocarmos $w$ por $w_{1 *}$ Entäo, devemos ter:

$$
\left\langle(w)-w_{1} \mid u\right\rangle=0, \quad \text { para todo } u \in D(B) .
$$

Uma vez que $D(B)$ b denso em $X$, concluímos que $w=w_{1}$, Assim o adjunto $B^{*}$ estrá unicamente definido $\{231,[24]$.

Teorema: Se um operador linear $B$ esta definido num domínio denso, entäa

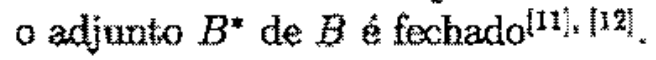

Definição: Sejam dois operadores $A$ e $B$ tal que $D(A) \supset D(B)$. Se no domínio de $B$, ocorrer que $A=B$ entāo $A$ é chamado de extensäo de $B$.

Definiçäo: Um operador linear $B$ e fechável se ele tem pelo menos una extensão fechada, A menor extensẵ fechada de um operador é chamada de seu fecho denotado por $\bar{B}$.

Definição: Un operador $B$ definido num domíno denso (do espaço de Hilbert) é chamado simétrico se $D(B) \subset D\left(B^{*}\right)$ e se $B \psi=B^{*} \mathcal{H}^{\prime}$, para todo Equivalenternente $B$ é simetrico se e so se:

$$
\langle\varphi \mid B \psi\rangle=\langle B \varphi \mid \psi\rangle \quad, \quad \forall \varphi, \psi \in D(B)
$$

Definição: Um operador linear $B$ é auto-adjunto se $B=B^{*}$, on seja se es só se $B$ é simétrico e se $D(B)=D\left(B^{*}\right)$.

Definiçăo: Um operador simétrico $B$ é chamado essencialmente azto-adjunto se seu fecho $\bar{B}$ at ato-adjunto. 


\subsection{Critério para possibilidade de extensōes auto-adjuntas.}

Se quisermos saber se um operador $B$ simétrico é auto-adjunto, devemos de acordo com o que foi definido na seção anterior, verificar se o domínto do operador $D(B)$ to mesmo de seu adjunto $D\left(B^{*}\right)$. No entanto, checar a identidade dos domínios $D(B)$ e $D\left(B^{*}\right)$ nảo é muitas vezes trivial. Existe um outro critério mais prático para se verificar se um operador é ou nāo auto-adjunto.

Suponhamas que um operador $B$ seja auto-adjunto e que exista um vetor $\varphi \in D\left(B^{*}\right)=D(B)$ tal que $B^{*} \varphi=i \varphi$. De modo que $B \varphi=i \varphi e$

$$
-i\langle\varphi \mid \varphi\rangle=\langle i \varphi \mid \varphi\rangle=\langle B \varphi \mid \varphi\rangle=\left\langle\varphi \mid B^{*} \varphi\right\rangle=\langle\varphi \mid B \varphi\rangle=i(\varphi|\varphi\rangle
$$

Entảo $\varphi=0$.

Assim se $B$ é auto-adjunto a equação $B^{*} \varphi=t$ ti $\varphi$ não possui solução não trivial. Do mesmo modo, pode ser verificado que se $B$ é um operador fechado e $B^{*} \varphi= \pm i \varphi$ nẽo tem solução, então $B$ é auto-adjunto.

Definiçäo: Chamamos de núcleo de um operador $F$, denotado por $K \operatorname{er}(F)$ ao conjunto de vetores no dominio $D(F)$, tal que:

$$
\operatorname{Ker}(F)=\{u \in D(F) \mid F u=0\} .
$$

Assim, o critério básico para se saber se um operador é auto-ajunto é:

Se trm operador $B$ é fechado e sé $\operatorname{Ker}\left(B^{*} \pm i\right)=\{0\}$, então $B$ é auto-adjunto. 
4.4. Técnica dos indices de deficiência de von Neumann para obtenção das extensōes auto-adjuntas de um operador simétrico.

A técnica dos índices de deficiência desenvolvida por won Neurnann permite verificarmos se um operador simétrico possui extensões auto-adjuntas, permitindo também a obtenção e caracterização dessaus extensões caso elas existam.

Na teoria de von Neumann os chamados subspaços de deficiência desempen-

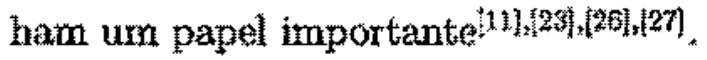

São chamados suthspaços de deficiência os seguintes subspaços de um operador simétrico $B$.

$$
\begin{aligned}
& D_{+}(B)=\operatorname{Ker}\left(i-B^{*}\right) \\
& D_{-}(B)=\operatorname{Ker}\left(i+B^{*}\right)
\end{aligned}
$$

Os indices de defciência de $B: n_{+}(B)$ e n-(B) säo definidos como sendo:

$$
\begin{aligned}
& n_{4}(B)=\operatorname{dim} D_{+}(B) \\
& n_{-}(B)=\operatorname{dim} D_{-}(B) .
\end{aligned}
$$

Através da introdução de um novo produto interno:

$$
\langle\varphi \mid \psi\rangle_{B}=\langle\varphi \mid \psi\rangle+\langle B \varphi \mid B \psi\rangle,
$$

pode ser demonstrado que o daxuínio do adjunto $B^{*}$ é dado pela soma direta dos seguintes subspacos mutuamente ortogonais com o produto interno definido acima ${ }^{[26,27,28] \text { : }}$

$$
D\left(B^{*}\right)=D(B) \oplus_{B} D_{+}(B) \oplus_{B} D_{-}(B) .
$$

Se o operador $B$ é simétrico em $D(B)$ e em $D_{+}(B) \oplus_{B} D_{-}(B)$ e sex

$$
n_{+}(B)=n_{-}(B)=n
$$

então, existe uma matriz unitária $U, n \times n$ que mapeia $D_{\text {s. }}(B)$ en $D_{-}(B)$. 
De modo que o domínio das extensöes auto-adjuntas é definido por:

$$
D(B)=\left\{\varphi+\varphi_{+}+U \varphi_{+} \mid \varphi \in D(B)_{i} \varphi_{+} \in D_{+}(B)\right\}
$$

e a ação do operador $B$ no domínio dado por (4.4.6) é:

$$
B\left(\varphi+\varphi_{+}+U \varphi_{+}\right)=B \varphi+i \varphi_{+}-i U \varphi_{+} .
$$

4.5. Relação entre as extensöes auto-adjuntas e as condiçöes de contorno.

Notemos que aplicaçāo de $U$ en um vetor $\varphi_{+} \in D_{+}(B)$ leva numa combinasăo linear de vetores $\phi_{\ldots} \in D_{-}(B)$, isto es:

$$
U \varphi_{+}^{i}=\sum_{j=1}^{n} W_{i j} \varphi_{-,}^{j}, i=1, \ldots, n
$$

Para encontrarmos as condiçōes de contorno a serem satisfeitas pelas funções $\psi$ no donínio das extensōes auto-adjuntas de $B$, basta utilizarmos o fato de que o operador $B$ é simétrico no domínio definido por $(4.4 .6)$, isto é:

$$
\left\langle B\left(\varphi_{+}^{i}+\sum_{j=1}^{n} W_{i j} \varphi_{-}^{j}\right) \mid \psi\right\rangle=\left\langle\left(\varphi_{+}^{i}+\sum_{j=1}^{n} W_{i j} \psi_{-}^{i}\right) \mid B \psi\right\rangle, \quad i=1, \ldots, n
$$

4.6. Obtençäo das extensōes auto-adjuntas para o operador momento linear em uma dimensäo.

Vamos considerar o operador momento linear $P=\frac{d}{d x}$ definido em $L^{2}(0,1)$. Para que $P$ seja simétrico em um domínio $D_{0}$ de funcôes shsolutamente contínuas no intervalo $(0,1)$ devemos ter que, para $\psi \in D_{0}(P)$ e $\varphi \in D_{0}\left(P^{*}\right)$;

$$
\begin{aligned}
\left\langle i \frac{d}{d x} \varphi \mid \psi\right\rangle & =\left\langle\varphi \mid i \frac{d}{d x} \psi\right\rangle \\
-\hat{i} \int_{0}^{1} \frac{d}{d x} \varphi^{*}(x) \psi(x) d x & =i \int_{0}^{1} \psi *(x) \frac{d}{d x} \psi(x) d x
\end{aligned}
$$


Integrando por partes vemos que isso só ocorre se:

$$
\varphi^{*}(1) \psi(1)-\varphi^{*}(0) \psi(0)=0
$$

Assim, é nexessúria a especificacão de condigóes de contonno para que o operador $P$ seja simétrico. No caso do operador momento linear em uma dimensão definido em $L^{2}(0,1)$ á facil encontrar as extensōes auto-ajdjuntas sem precisar utilizar a túcnica dos indices de deficiência. Se definirmos $D_{0}(P)$ como sendo:

$$
D_{0}(P)=\{\psi \mid \psi \in A C[0,1], \psi(0)=\psi(1)=0\}
$$

então o operador $P$ é simétrico. No entanto, a condicâa de contorno para as funçoes em $D_{0}(P)$ acima $(\psi(0)=\psi(1)=0)$ é "muito forte" necessário irnpor qualquer condiçăo de contorno às funções em $D_{0}\left(P^{*}\right)$ para que a tuação $(4.6 .2)$ se verificue.

Assim:

$$
D_{0}\left(P^{*}\right) \supset D_{0}(P)
$$

Para definirmos o operador $P$ corno sendo um operador auto-adjunto é necessário impor condig öes de contorno mais gerais (ou mais "fracas ") a serem obedecidas pelas funçôes am $D(P)$ tais que (4.6.2) rexueira as mesmas condiçóes de contorno para as funçöes em $D\left(P^{*}\right)$, isto é, $D(P)=D\left(P^{*}\right)$.

Podemos facilmente verificar que definindo o domínio de $P$ como sendo

$$
D(P)=\{\psi|\psi \in A| 0,1], \psi(1)=\alpha \psi(0)|\alpha \in C,| \alpha \mid=1\}
$$

a equagäo (4.6.2) implica em mesmas condiçöes de contorno a serem satisfeitas pelas funçoes em $D\left(P^{*}\right)$, isto é $D\left(P^{*}\right)=D(P)$, de modo que o operador $P$ definido nesse domínio $\mathrm{E}$ anto-adjunto. A cada valor de $\alpha$ corresponde uma extensäo autoadjunta para o operador $P$.

Vamos agora aplicar a técnica dos indices de deficiencia de von Neumann para verificarnos que é possivel chegar ao mesmo domínio obtido através da equagäo $(4.6 .5)$. 
Vamos começar determinando os subspaģos de deficiencia $D_{4}(P)$ e $D$. $(P)$ definidos por:

$$
\begin{aligned}
& P^{*} \varphi_{t}= \pm i \varphi_{\text {s }} \text { se } \varphi_{ \pm} \in D_{t} \Rightarrow \\
& \frac{d}{d x} \varphi_{ \pm}= \pm i \varphi_{\text {s }} \text { se } \varphi_{ \pm} \in D_{ \pm}
\end{aligned}
$$

Assim $D_{ \pm}(P)=\left\{c e^{t} \mid c \in C\right\}$, de modo que os indices de deficiencia $n_{+}(P)$ e $n_{-}(P)$ são ambos iguats a um. E portanto existe uma familì a um parâtnetro de extensōes zuto-adjuntas admissiveis

Considerando os vetores mormalizados de $D_{ \pm}(P)$ :

$$
\varphi_{+}=\frac{\sqrt{2}}{\sqrt{e^{2}-1}} e^{*} \text { e } \varphi_{-}=\frac{\sqrt{2 e}}{\sqrt{e^{2}-1}} e^{-x}
$$

vemos que as únicas aplicaçóc unitárias de $D_{+}(P)$ ein $D_{-}(P)$ são as dadas por $\varphi_{+} \rightarrow e^{i \theta} \varphi$ com $\theta \in R$. De ruodo que as extensōes auto-adjuntas de $P$ são dadas pelo domínio:

$$
D_{\theta}(P)=\left\{\psi+\beta \varphi_{+}+e^{a} \beta p_{-} \mid p \in D(P), \beta \in C\right\}
$$

E interessante checarmos que o dominio dado por (4.6.9) 6 o mesmo que o da exuacão (4.6.5). Para isso basta usarmos o fato de que o operador $P$ deve ser simétrico no domínio de suas extensöes auto adjuntas, recorrendo à equagăa (4.5.2). Assim, seja $\psi \in D_{Q}(P)$ entào, devernos ter que:

$$
\begin{aligned}
& \left\langle P\left(\varphi_{+}+e^{i \theta} \varphi_{-}\right) \mid \psi\right\rangle=\left\langle\varphi_{+}+e^{i \varphi} \varphi_{-}\right)|P \psi\rangle \\
& -i \int_{0}^{1} \frac{d}{d x}\left(\frac{\sqrt{2}}{\sqrt{e^{2}-1}} e^{x}+e^{\cdots i \theta} \frac{\sqrt{2} e}{\sqrt{e^{2}-1}} e^{-2}\right) \psi(x) d x= \\
& -i \int_{0}^{1}\left(\frac{\sqrt{2}}{\sqrt{e^{2}-1}} e^{x}+e^{-i t} \frac{\sqrt{2} e}{\sqrt{e^{2}-1}} e^{-4}\right) \frac{d}{d x} \psi(x) d x
\end{aligned}
$$


Para que a igualdade acima se verifique devemos ter a satísfação da equação (4.6.2) que nesse caso se torna:

$$
\frac{\sqrt{2}}{\sqrt{e^{2}-1}}\left[\left(e+e^{-i \theta}\right) \psi(1)-\left(1+e^{-i \theta} e\right) \psi(0)\right]=0
$$

A equaçäo acima dá a seguinte condị̧ăo de contorno para as funçồ $y$ no domínio das extensōes anto-adjuntas:

$$
\psi(1)=\alpha \psi(0)
$$

onde:

$$
\alpha=\frac{1+e^{-i \phi} e}{e+e^{-i \phi}}, \text { com }|\alpha|=1
$$

Portanto, como vemos, as condiçōes de contorno obtidas através da equaçäo (4.6.11) para as funçós $\psi$ do domínio $D_{\theta}(P)$ dado pela equação (4.6.9) são as mesmas condições de contorno descritss pela equação $(4.6 .5)$ e definem os domínios para us quais operador $P$ é auto-adjunto. Para cada valor de $\alpha$ na equação (4.6.13) é possivel uma extensão auto-adjunta (corn $|\alpha|=1)$ para $P$ definido em $L^{2}(0,1)$.

4.7. Obtenção das extensöes auto-adjuntas para o operador energia cinética definido em $L^{2}(0, \infty)$ em uma dimensão.

Varnos considerar o operador energia cinética $K=-\frac{d^{2}}{d t^{2}}$, definido no domínio $D_{0}(K)$ constituído pelas funçöes $A C(0, \infty)$ e infinitamente diferenciáveis.

Vamos inicialmente aplicar o método dos índices de deficiência para determinar as extensões anto-adjuntas desse operador.

As únicas soluçōes normalizáveis da equação:

$$
\frac{-d^{2} \hat{\varphi}}{d x^{2}}= \pm i \varphi_{ \pm}
$$

งส๊ก:

$$
\begin{aligned}
& \varphi_{+}=\frac{1}{N} e^{\frac{t(-1+i)}{\sqrt{2}} x}, \\
& \varphi_{-}=\frac{1}{N} e^{\frac{-\mid t+4 x_{x}}{\sqrt{n}}},
\end{aligned}
$$


uma vez que as soluçōes $\varphi_{+}=e^{\frac{(1-y)}{\sqrt{2}}+}$ e $\varphi_{-}=e^{\frac{ \pm 1++i)}{\sqrt{2}}}$ näo sâo de quadrado inteyrável no intervalo $(0, \infty)$, ou seja, nāo pertencem a $L^{2}(0, \infty)$.

Sendo assim os índices de defleiência $n_{+}(K)$ e $n_{-}(K)$ são ambos ìguais a un a as ínicas aplicaço un untárias de $D_{+}(K) \mathrm{em} D_{-}(K)$ são dadas por $\varphi_{+} \rightarrow e^{i \| \varphi_{-}}$. De modo que as extensöes auto-adjuntas são dadas pelo domínio:

$$
D_{0}(K)=\left\{\varphi+\beta \varphi+e^{i \theta} \beta \varphi \mid \varphi \in D_{0}(R), \beta \in C\right\}
$$

Notemos que, para que o operador $K=\frac{-d^{2}}{d^{2}}$ definito em $L^{2}(0, \infty)$ seja simétrico num domínio $D_{0}(K)$ de funcöes $A C(0, \infty)$ e infinitamente diferenciáveis que se anulam no infinito, devermos ter que, para $\psi \in D_{0}(K)$ e $\varphi \in D_{0}\left(K^{*}\right)$ :

$$
\left\langle\frac{-d^{2} \phi}{d x^{2}} \mid \psi\right\rangle=\left(p \mid \frac{-d^{2} \psi}{d x^{2}}\right)
$$

A integraça por partes nos mostra que a igualdade acima só é satisfeita se :

$$
\varphi^{*}(0) \frac{d w}{d x}(0)-\frac{d \varphi^{*}}{d x}(0) \psi(0)=0
$$

Se quisermos encontrar as condições de contorno a serem satisfeitas pelas furçōes no domínio das extensōes auto-adjuntas do operador $K$ definido em $l^{2}(0, \infty)$, basta usarmos of fato de que tal operador $K$ deve ser simétrico no domínio $D_{b}(K)$ de suas extensöes auto-adjuntas, recorrendo suação (4.5.2). Assim, para uma funça $\psi \in D_{f}(K)$ devemos ter que:

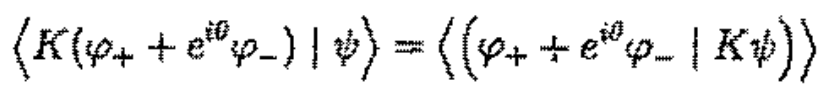

Para que a igualdade acima se verifique devemos ter a satistação da equação (4.7.6), que nesse caso, nos leva a :

$$
\left(1+e^{-i \theta}\right) \frac{d \psi t}{d x}(0)+\left[e^{i \frac{x}{4}}+e^{-i \theta} e^{-i \pi}\right] \psi(0)=0
$$


A equaçăo acima dá a seguinte condição de contono para as funções $\dot{\psi} \in$ $D_{\theta}(K):$

$$
\frac{d \psi}{d x}(0)=\alpha \psi(0)
$$

onde:

$$
\alpha=\frac{\cos \left(\frac{\theta}{2}+\frac{\pi}{4}\right)}{\cos \left(\frac{\theta}{2}\right)}
$$

on seja $\alpha \in R-\{\infty\}$.

Para $\alpha=\infty$, isto $\hat{e}$, quando o parâmetro $\theta$ vale $\pi$, a equaçăo (4.7.8) fornece a seguinte condição de contorno:

$$
\psi(0)=0
$$

Assim, para cada valor de $\alpha \in R$ na equaçäo (4.7.9), é possivel uma extensäo auto-adjunta para o operador $K$ definido em $L^{2}(0, \infty)$.

\subsection{Obtenção das extensöes auto-adjuntas para o operador energia cinética definido em $L^{2}(-\infty,+\infty)$ em una dimensão.}

Vamos considerar agora o problema de determinaçân das extensöes auto-adjuntas do operador energia cinética $K=-\frac{d^{2}}{d x^{2}}$, definido em $L^{2}(-\infty,+\infty)$. Nesse caso a obtençăo das condiçōes de contorno mais gerais nāo é tão simples como nos casos tratados anteriormente. Assim, convem encontrarmos as extensöes auto-adjuntas diretamente através do método dos índices de deficiência e utilizando a fórmula da equação (4.5.2). No entanto, exse problema nầo trivial já foi considerado pelos autores Carreau ${ }^{[3]}$ e por Abeverio ${ }^{[14]}$. De forma que, é interessante checarmos se conseguimos reproduzir os resultados das referências [9], [13] e [14] através da fórmula da equaçāo (4.5.2).

Para que o operador $K$ definido em $L^{2}(-\infty,+\infty)$ seja simétrico devemos ter que para $\psi$ no domínio de $K$ e $\phi$ no domínio do adjunto $K *$ :

$$
\left\langle-\frac{d^{2}}{d x^{2}} \phi \mid \psi\right\rangle=\left\langle\phi 1-\frac{d^{2}}{d x^{2}} \psi\right\rangle
$$


A integração por partes nos mostra que a igualdade acima só é satisfeita se:

$$
\left.\omega^{*} \psi^{*}-\phi^{* t} \psi\right]_{0^{-}}^{0^{*}}=0
$$

Os subspagos de deficiencia $D_{ \pm}(K)$ definidos pelas aguações:

$$
-\frac{d^{2}}{d x^{2}} \varphi_{ \pm}= \pm i \varphi_{ \pm} \quad, \quad \varphi_{ \pm} \in D_{ \pm}(K)
$$

são gerados pelas funçōes ortonormalizadas descontínuas na origem:

$$
p_{+}^{1}=\left\{\begin{array}{cc}
0 & , x<0 \\
e^{\frac{-1+x^{2} x}{\sqrt{2}}}, & , x>0
\end{array}\right\} \quad p_{+}^{2}=\left\{\begin{array}{cc}
e^{\frac{1-1}{\sqrt{2} x}}, & x<0 \\
0, & x>0
\end{array}\right\}
$$

a:

$$
\varphi_{m}^{2}=\left\{\begin{array}{cc}
0, & x<0 \\
e^{\frac{-1-i}{\sqrt{2}}}, & , x>0
\end{array}\right\} \quad \varphi_{-}^{2}=\left\{\begin{array}{cc}
e^{\frac{4+i}{\sqrt{2}} z}, & x<0 \\
0, & x>0
\end{array}\right\}
$$

Vemos entâo que as dinaentöos dos subspaços de defieiencia $D_{ \pm}(K)$, isto é, os índices de deficiencia $n_{ \pm}(K)$ säo ambos iguais a dois. $\mathrm{E}$ portanto, as condicon de contorro admissiveis para os vetores $\psi$ no domínio das extensöes auto-adjuntas do operador simétrico $K$, podem ser parametrizadas por uma matriz unitária $W$ $2 \times 2$, atrave das equacoin:

$$
\left(K\left(\varphi_{+}^{1}+w_{11} \varphi_{-}^{1}+w_{12} \varphi_{-}^{2}\right) \mid 1 /\right)=\left\langle\left(\varphi_{+}^{1}+w_{11} \varphi_{-}^{1}+w_{12} \varphi_{-m}^{2}\right) \mid K \psi\right\rangle
$$

a:

$$
\left.\left\langle K\left(\varphi_{+}^{2}+w_{21} \varphi_{-}^{2}+w_{22} \varphi_{-}^{2}\right)\right| \psi\right)=\left\langle\left(\varphi_{+}^{2}+w_{21} \varphi_{-}^{1}+w_{22} \varphi_{w}^{2}\right)|K \psi| k\right\rangle
$$

A matriz unitária $W$ pode ser tomada como tendo a forme particular:

$$
W=\left(\begin{array}{cc}
\cos b e^{i(t+a)} & i \operatorname{sen} b e^{-i(\xi)-a)} \\
i \operatorname{sen} b e^{i(t+a)} & \cos b e^{-i(c-a)}
\end{array}\right) .
$$


Usendo equaçôtes $(4.8 .2),(4.8 .4)$, e $(4.8 .5)$ as equaçóes $(4.8 .6)$ e $(4.8 .7)$ acima se tornam:

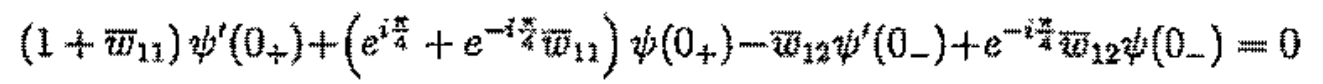

e:

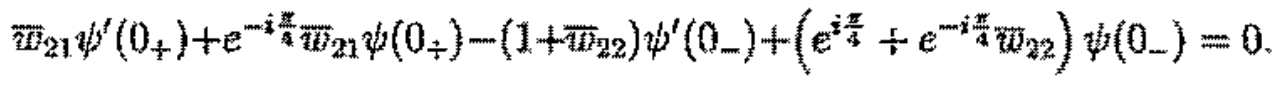

As equaçes $(4.8 .9)$ e (4.8.10) adima com a matriz $W$ dada pela equação $(4.8 .8)$ fornecem as condiçoes de contorno mais geraís a serem satisfeltats pelas funcỏes de onda na vizinhança da origem, correspondendo ao domínio mais geral, onde o operador $K$ (definido em $L^{2}(-\infty,+\infty)$ ) é auto-adjunto.

No entanto, como dissemos anteriormente, as condiçow de contorno mais gerais para esse problema do operador energia cinética já foram encontradas por Carreau [i3] e por Albeveriollin. As condiço de contorno mais gerais obtidas por esses uutores podem ser escritas como na referència [8] como sendo.

$$
\left(\begin{array}{l}
\psi^{\prime}\left(0_{+}\right) \\
\psi\left(0_{+}\right)
\end{array}\right)=U\left(\begin{array}{l}
\psi\left(0_{-}\right) \\
\psi\left(0_{-}\right)
\end{array}\right)
$$

onde $\psi^{\prime}(x)=\frac{d^{\prime}(x)}{d x} \mathrm{e}$ :

$$
U=e^{i \theta}\left(\begin{array}{ll}
\alpha & \beta \\
\delta & \gamma
\end{array}\right)
$$

onde os parâmetros $\alpha, \beta, \delta, \gamma$ a säo constantes reais satisfazendo a seguinte relação de unitariedade:

$$
\alpha \gamma-\beta \delta=1
$$


Vamos verificar se as condiçōes de contorno obtidas atravếs das equacõos $(4.8 .9)$ e (4.8.10) coincidern com as referênicias [8], [13] e [14].

Multiplicando a equação (4.8.9) pelo fator $e^{-i \frac{\pi}{4} \text { Tw }_{21}}$ e a equaçäo $(4.8 .10)$ pelo fator $e^{i \frac{\pi}{4}}\left(1-i \varpi_{11}\right)$ e subtraindo as equaçoes assim obtidas, temos:

$$
\begin{gathered}
\bar{w}_{21}\left[e^{-i \frac{\pi}{4}}\left(1+\bar{w}_{11}\right)-e^{i \frac{\pi}{4}}\left(1-i \bar{w}_{11}\right)\right] \psi^{z}\left(0_{+}\right) \\
+\left[e^{i \frac{\pi}{4}}\left(1-i \bar{w}_{11}\right)\left(1+\bar{w}_{22}\right)-e^{-i \frac{\pi}{4} \bar{w}_{12} \bar{w}_{21}}\right] \psi^{\prime}\left(0_{-}\right) \\
-i\left[\bar{w}_{12} \bar{w}_{21}+\left(1-i \bar{w}_{11}\right)\left(1-i \bar{w}_{22}\right)\right] \psi\left(0_{-}\right)=0 .
\end{gathered}
$$

Vtilizando a matriz unitária $W$ como sendo dada pela equaçào (4.8.8), a equaçäo (4.8.14) acima, para, senb diferente de 0 (isto é $w_{12}$ e w $w_{21}$ diferentes de zero), se torna:

$$
\begin{aligned}
\psi^{\prime}\left(0_{4}\right)= & e^{i \phi}\left[\sqrt{2}\left(\frac{\cos \left(a+\frac{\pi}{4}\right)-\cos b \operatorname{sen}\left(c-\frac{\pi}{4}\right)}{\operatorname{sen} b}\right)\right] \psi^{\prime}\left(0_{-}\right) \\
& +e^{\omega \phi}\left[\sqrt{2}\left(\frac{\operatorname{sen} a-\cos b \cos c}{\operatorname{sen} b}\right)\right] \psi\left(0_{-}\right) .
\end{aligned}
$$

Comparando a equação acima com a equaçäo $(4.8 .11)$, podemos identificar os parâmetros $\alpha$ e $\beta$ e $\theta$ da referência [8] em função dos parâmetros $\psi$, $a, b$ e $c$ da equaçăo (4.8.8):

$$
\begin{gathered}
\alpha=\sqrt{2}\left(\frac{\cos \left(a+\frac{\pi}{4}\right)-\cos b \operatorname{sen}\left(c-\frac{\pi}{4}\right)}{\operatorname{sen} b}\right), \\
\beta=\sqrt{2}\left(\frac{\operatorname{sen} a-\cos b \cos c}{\operatorname{sen} b}\right)
\end{gathered}
$$

e:

$$
\theta=\psi
$$


Multiplicando a equaçāo (4.8.9) pelo fator $\bar{w}_{21}$ e a equaça (4.8.10) pelo fator $\left(1+\bar{w}_{11}\right)$ e depois subtraindo as equaçös assim obtidas, temos:

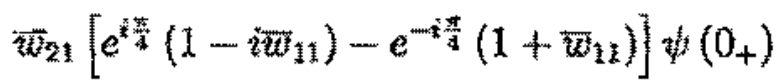

$$
\begin{aligned}
& +\left[\left(1+\bar{w}_{11}\right)\left(1+\bar{w}_{22}\right)-\bar{w}_{12} \bar{w}_{21}\right] \psi^{*}\left(0_{-}\right)
\end{aligned}
$$

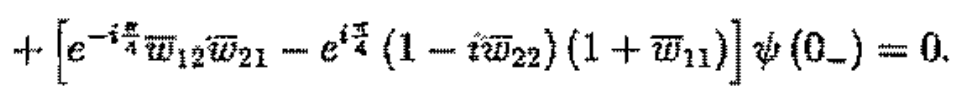

Novamente, utilizando a equaça (4.8.8), a equaçäo (4.8.17) acima, para senb diferente de 0 (isto é $w_{12}$ e $w_{21}$ diferentes de zero), se torna:

$$
\begin{array}{r}
\psi\left(0_{4}\right)=-e^{i \psi}\left[\sqrt{2}\left(\frac{\cos a+\cos b \cos c}{\operatorname{sen} b}\right)\right] \psi\left(0_{-}\right) \\
+e^{i \psi t}\left[\sqrt{2}\left(\frac{\cos \left(a+\frac{\pi}{4}\right)+\cos b \operatorname{sen}\left(c+\frac{\pi}{4}\right)}{\operatorname{sen} b}\right)\right] \psi\left(0_{-}\right)
\end{array}
$$

Comparando a equaçäo acima com a equação (4.8.11) poderno identificar as seguintes relaçoes entre os parametros $\beta, \delta$ e das referencias [8], [9],[13] e [14]. com os parâmetros $\psi, a, b$ e $c$ da equaçăo $(4,8.8)$ :

$$
\begin{gathered}
\delta=-\sqrt{2}\left[\frac{\cos a+\cos b \cos c}{\operatorname{sen} b}\right] \\
\gamma=\sqrt{2}\left[\frac{\cos \left(a+\frac{\pi}{4}\right)+\cos b \operatorname{sen}\left(c+\frac{\pi}{4}\right)}{\operatorname{sen} b}\right]
\end{gathered}
$$

e.

$$
a=\psi
$$

É fácil verificar que os parămetros $\alpha, \beta, \delta$ e $\gamma$ escritos em funcöes doc três parâmetros $a, b$ ec através das equaçoes $(4.8 .16 \mathrm{a})$ e (4.8.16b) e $(4.8 .19 \mathrm{a})$ e (4.8.19b) satisfazem automaticamente a equação $(4.8 .13)$ da unitariedade. 
Para o caso em que $w_{12}=w_{21}=0$ (isto é senb $=0$ ), as condicóes de contorno das equaçōes (4.8.14) e (4.8.17) se tornam:

$$
\psi^{\prime}\left(0_{ \pm}\right)=\eta_{ \pm} \psi^{\prime}\left(0_{ \pm 2}\right) \quad, \eta_{ \pm} \in R
$$

De forma que as condições de contorno obtidas através da imposição das equaçós (4.8.6) e (4.8.7), onde a matriz paramétrica $W$ b dada pela equaçäo (4.8.8), são as mais gerais para o operador energia cinética em uma dimensäo definido em $L^{2}(-\infty,+\infty)$ e coincidem com as demais apresentadas na literatura $[8],[9],[13]$ \& $[14]$. 


\section{Capítulo 5}

\section{Extensões auto- adjuntas a dois parâmetros que nāo acoplam todas as componentes das funçōes de onda.}

\subsection{Extensões Auto-adjuntas para os operadores $H$ e $A$.}

O problema da determinaçăo das extensōes auto-adjuntas de um operador simétrico definido num domínio denso é equivalente ao problema da determinaçẵo das condiçoes de contomo admissiveis para o mesmo conforme vimos (atravé dos exemplos) no capitulo anterior.

A tenica que usaremos para esse fim ju fol descrita no Capituio 4 e requer a determinaçào das espaços de deficiencia dos operadores $H$ e $A$ simétricos, atuando num dornínio denso (do espaço de Hilbert) de huncones continuas ern $r$ que se anulam na origem.

Os subspaços de deficiência $D_{ \pm}(H)$ e $D_{ \pm}(\Lambda)$ säo definidos por:

$$
\begin{gathered}
H^{*} \varphi_{ \pm}= \pm i m \varphi_{ \pm}, \quad \text { se } \varphi_{ \pm} \in D_{ \pm}(\Lambda), \\
\Lambda^{*} \xi_{ \pm}= \pm i l \xi_{ \pm}, \quad \text { se } \xi_{ \pm} \in D_{ \pm}(\Lambda),
\end{gathered}
$$

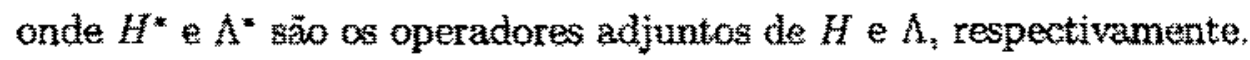

Inicialmente varnos encontrar a funcoes $p_{\text {s. }}$ dadas pela equacão (5.1.1a), do subspago $D_{ \pm}(H)$.

Usando que $H=H^{*}$, ou seja a agäo dos operadores $H$ e $H^{*}$ e mesma (operador simétrico) e que:

$$
H=\left(\begin{array}{cc}
h+\sigma_{3} m & 0 \\
0 & h-\sigma_{3} m
\end{array}\right)
$$


(veja equação (2.2.14)), a exuatuço (5.1.1) se torna:

$$
\left(\begin{array}{cc}
h+\sigma_{3} m & 0 \\
0 & h-\sigma_{3} m
\end{array}\right)\left(\begin{array}{l}
\phi_{ \pm}^{2} \\
\phi_{ \pm}^{2}
\end{array}\right)=\operatorname{sim}\left(\begin{array}{l}
\phi_{ \pm}^{2} \\
\phi_{ \pm}^{2}
\end{array}\right),
$$

onde introduzimos:

$$
\varphi_{ \pm}=\left(\begin{array}{c}
\phi^{1} \\
\phi^{2}
\end{array}\right)
$$

com $\phi_{ \pm}^{1}$ e $\phi_{ \pm}^{2}$ sendo espinores de duas componentes.

Resolver o problema de anto-valores acima (de quatro componentes) é equivalente a resolver o seguinte problema (de duas componentes):

$$
\left(h+m s \sigma_{3}\right) \phi_{ \pm}^{j}= \pm i m \phi_{ \pm}^{j}, j=1,2,
$$

onde para $s=1$ estamos resolvendo o problema da deterninação de $\phi \pm$ na equagäo (5.1.4) e $s=-1$ corresponde a determinaca de $\phi^{2}$.

Lembrando da equacăa $(2.2 .15)$ e definindo:

$$
\hat{\phi}_{ \pm}^{j}=\left(\begin{array}{c}
v_{+}^{j} \\
v_{ \pm}^{j} \\
v_{ \pm}
\end{array}\right)
$$

a. equaça (5.1.5) se torna:

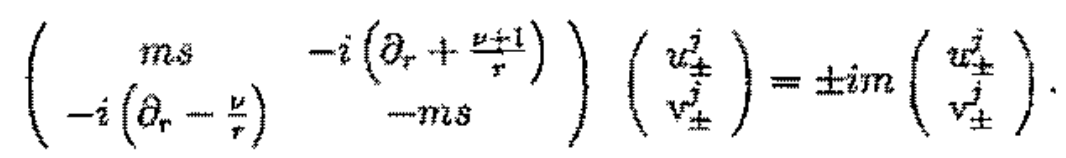

Da equagao (5.1.7) temos:

$$
\begin{aligned}
& m s u_{t}^{z}-i\left(\partial_{\mathrm{r}}+\frac{w+1}{t}\right) \mathrm{y}_{ \pm}^{j}= \pm m u_{t}^{i} \\
& -i\left(\partial_{r}-\frac{v}{r}\right) u_{ \pm}^{j}-s m v_{ \pm}^{j}= \pm i m v_{ \pm}^{j}
\end{aligned}
$$

Da equatäo (3.1.9) temos:

$$
v^{j}=\frac{-i\left(\partial_{r}-\frac{\nu}{r}\right) u_{ \pm}^{j}}{( \pm i m+s m)}
$$


Substituindo a equação (5.1.10) na exuação (5.1.8) temos:

$$
\partial_{r}^{2} u_{ \pm}^{i}+\frac{1}{r} \partial_{r} u^{3}-\left(\frac{\nu^{2}}{r^{2}}+2 m^{2}\right) w_{z}^{3}=0
$$

A equaģăo acima pode ser escrita como:

$$
\partial_{\sqrt{2} m r^{2}}^{2}+\frac{1}{\sqrt{2} m r} \partial_{\sqrt{2 m} m^{4} t_{ \pm}^{4}}-\left(1+\frac{\nu^{2}}{(\sqrt{2 m r})^{2}}\right) u_{ \pm}^{3}=0
$$

cuja solugäo é:

$$
u_{ \pm}(\sqrt{2} m \tau)=K_{y}(\sqrt{2} m r)
$$

Voltando com esse resultado na exuacäo (5.1.10) podemos determinar $v_{ \pm}^{4}$ :

$$
v_{ \pm}^{j}=-i \frac{\sqrt{2}}{ \pm i+s}\left(\partial_{\sqrt{2 m} r}-\frac{y}{\sqrt{2} m r}\right) K_{t}(\sqrt{2 m r})
$$

Mas comoliol:

$$
\partial_{x} K_{\nu}-\frac{\nu}{x} K_{z}=-K_{\nu+1}
$$

a equação (3.1.13) finalmente fornece:

$$
v_{ \pm}^{j}=\frac{i \sqrt{2}}{ \pm+i+s} K_{v+1}(\sqrt{2} m \tau)
$$

Assim podemos escrever as funçōes $\phi_{ \pm}^{(1,2)}$ da equação (5.1.6) como sendo:

$$
\phi_{ \pm}^{(1)}=\frac{1}{N}\left[\begin{array}{c}
K_{\nu}(\sqrt{2} m r) \\
\pm e^{ \pm i \frac{\pi}{4}} K_{z+1+1}(\sqrt{2} m r)
\end{array}\right]
$$

e:

$$
\phi_{i}^{(2)}=\frac{1}{N}\left[\begin{array}{c}
K_{\nu}(\sqrt{2} m r) \\
2 e^{\mp i \frac{\pi}{i}} K_{\nu+k}(\sqrt{2} m r)
\end{array}\right]
$$

onde $N$ uma constante de normalização. 
Assim estäo determinadas as finçöes $\varphi_{\text {f }}$ ortonormalizadas dos subspagos de deficiêneias $D_{\text {出 }}(H)$ da equação $(5.1 .1)$ :

e:

$$
\varphi_{+}^{(1)}=\left[\begin{array}{c}
\phi_{+}^{(1)} \\
0
\end{array}\right] \quad, \quad \varphi_{m}^{(1)}=\left[\begin{array}{c}
\phi_{-}^{(1)} \\
0
\end{array}\right]
$$

$$
\varphi_{+}^{(2)}=\left[\begin{array}{c}
0 \\
\phi_{+}^{(2)}
\end{array}\right] \quad, \quad \phi_{-}^{(2)}=\left[\begin{array}{c}
0 \\
\phi_{-}^{(2)}
\end{array}\right] .
$$

Se ollharmos $H$ da equaçäo (5.1.4) como um par de operadores desacoplados atuando em espinores de duas componentes, entäo, vemos que, os indices de deficiência são iguaỉs a um para cada operador do par.

Para determinarmos os findices de deficiência de $A$, vamos nos lembrar que $A$ pode ser obtido através da transformagäo da unitária da equacto (2.1.18) abaixo tratuscrita:

$$
\Lambda=U(H-\beta m) U^{-1}
$$

A equação (5.1.21) implica que o problema de determinação dos índices de deficiência de $\Lambda$ é equivalente ao problema de determinaçâo dos indices de deficiencia de $H-B m$.

Assim, nós devenos determinar as subspaços de deficiencia de $H-\beta m$, resolvendo a equacäo:

$$
(H-\beta m) \chi_{ \pm}= \pm i l \chi_{ \pm} \quad \chi_{ \pm} \in D_{ \pm}(H-\beta m) .
$$

Lembrando das definições de $\beta$ da equação (2.1.6) e de $H$ das equaçōes (2.1.1) e (2.2.7), o operador $H-\beta m$ pode ser escrito como sendo o operador diagonal:

$$
H-\beta m=\left[\begin{array}{cc}
h & 0 \\
0 & h
\end{array}\right]
$$

De modo que, os espinores $\chi_{ \pm}$(de quatro componentes) da equação (5.1.22) podem ser obtidos, resolvendo-se primeiro o seguinte problema de duas componentes:

$$
h \phi_{\text {A }}= \pm i l \phi_{ \pm x}
$$

Vamas introduzir a definiç̧ăo:

$$
\phi_{ \pm}=\left(\begin{array}{c}
u_{ \pm} \\
v_{ \pm}
\end{array}\right)
$$


Usando a equaçăo (5.1.25) a equagăo (5.1.24) se torna:

$$
\left(\begin{array}{cc}
0 & -\hat{k}\left(\partial_{t}+\frac{\mu+1}{r}\right) \\
-i\left(\partial_{r}-\frac{\mu}{r}\right) & 0
\end{array}\right)\left(\begin{array}{l}
u_{ \pm} \\
v_{ \pm}
\end{array}\right)= \pm i l\left(\begin{array}{l}
u_{z_{2}} \\
v_{ \pm}
\end{array}\right)
$$

Da equaçāo $(5.1 .25)$ temos as seguintes equaços que relacionam $u_{\text {. }}$ e $v_{ \pm}$:

$$
\begin{aligned}
& -\left(\partial_{r}+\frac{\nu+1}{r}\right) v_{ \pm}= \pm i l u_{ \pm} \\
& -\left(\partial_{r}-\frac{v}{r}\right) u_{ \pm}= \pm i v_{ \pm} .
\end{aligned}
$$

Substituindo a equach $(5.1 .28)$ na equação $(5.1 .27)$, vern que:

$$
\left(\partial_{l r}^{2}+\frac{1}{l p} \partial_{l r}\right) u_{ \pm}-\left(1+\frac{\nu^{2}}{(l n)^{2}}\right) u_{ \pm}=0
$$

cuja soluça

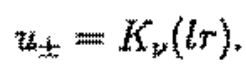

Voltando com o resultado da equaçäo acima na equaç2̃o (5.1.28), temos:

$$
\mathrm{v}_{s}=\left(\partial_{l r}-\frac{u}{l r}\right) K_{w}(l r)
$$

Usando o resultado da equação (5.1.15) concluimos que:

$$
v^{1}= \pm K_{2 r+1}(l r) \text {. }
$$

Assim, estäo agora também determinadas ass funcōes ortogonass $\chi_{ \pm}$(de quatro componentes) dos subspaşos de defictêncla $D_{ \pm}(H-\beta m)$

$$
\begin{array}{ll}
\chi_{+}^{(1)}=\left[\begin{array}{c}
\phi_{+} \\
0
\end{array}\right] \quad, \quad \chi_{-}^{(1)}=\left[\begin{array}{c}
\phi_{-} \\
0
\end{array}\right], \\
\chi_{+}^{(0)}=\left[\begin{array}{c}
0 \\
\phi_{+}
\end{array}\right] \quad, \quad \chi_{-}^{(2)}=\left[\begin{array}{c}
0 \\
\phi_{-}
\end{array}\right],
\end{array}
$$


onde:

$$
\phi 土=\frac{1}{N}\left[\begin{array}{c}
K_{v}(l r) \\
\pm K_{v+1}(l r)
\end{array}\right]
$$

Dessa forma, vemos que a dimensầ dos subspaços $D_{ \pm}(H-\not m)$, isto 6 , os índices de deficiência $d_{: t}(H-[\mathrm{m})$ são iguais a dois. Devido a relação dada pela equaçäo (2.1.18) tambérn podemos dizer que isso significa que $d_{ \pm}(\Lambda)=2$. As funçóes $\xi_{ \pm}$dos subspagos de deficiência $D_{ \pm}(\Lambda)$ são obtidas pela aplicação de $U$ nas auto-funçôes $\chi_{ \pm}$, isto é: $U \chi_{ \pm}^{(1)}$ e $U_{\chi \pm}^{(2)}$.

Da teoria geral, ja apresentada e discutida na Seçăo 4.5 , as condiçôes de contorno admissiveis para os vetores $\psi$, nos domínios das extenscos anto-adjuntas de um operador simétrico $A$ com iguais indices de deficiência $d_{+}(A)=L_{-}(A)=n$, săo parametrizadas por una matriz unitária $W, n \times n$, atravês da equaçāo (4.5.2) abaixo transcrita:

$$
\left\langle A^{*}\left[f_{+}^{j}+\sum_{k=1}^{n} w_{k_{k}} f_{-}^{k}\right] \mid \psi\right\rangle-\left\langle\left[f_{+}^{j}+\sum_{k=1}^{n} w_{j k} f_{-}^{k}\right] \mid A^{*} \psi\right\rangle=0,
$$

onde $f_{ \pm}^{j}$ são os vetores ortonormalizados em $D_{ \pm}(A)$, tais que:

$$
A^{*} f_{ \pm}= \pm i a f^{j} \quad \text { com } \quad j=1, \ldots n .
$$

Assim as condiçōes de contorno para $H$ estão parametrizadas por uma matriz $2 \times 2$ unitária:

$$
W=\left[\begin{array}{ll}
w_{11} & w_{12} \\
w_{21} & w_{92}
\end{array}\right]
$$

através de um conjunto de duas expaç̄öes que devemos encontrar a partir da equaça (5.1.35). Para isso, vejamos que a equaçäo (5.1.35) para o operador $H$ pode ser escrita na forma:

$$
(H \phi|\psi\rangle=\langle\phi \mid H \psi\rangle
$$

onde:

$$
\phi=\varphi_{+}^{i}+\sum_{k=1}^{n} w_{j k} \varphi_{-}^{k} \quad, \quad j=1,2
$$


Dessa forma, vernos que na equaça (5.1.38) estamos requerendo que o operador $H$ seja simétrico nos domínios de suas extensozes auto-adjuntas. Lembrando que o operador $H$ pode ser escrito corno na equaçäo (2.2.14), isto é:

$$
H=\left(\begin{array}{cc}
h & 0 \\
0 & h
\end{array}\right)+\left(\begin{array}{cc}
\sigma_{3} m & 0 \\
0 & -\sigma_{3} m
\end{array}\right)
$$

onde, obviamente, a segunda parcela corresponde a um operador simétrico, o problema se reduz a encontrar a condiçãó necessária para que o operador:

$$
\bar{H}=\left(\begin{array}{ll}
h & 0 \\
0 & h
\end{array}\right)
$$

seja simêtrico.

Lembrando da definiçăo de $h$ (equaçẫo (2.2.15)), o operador hamiltoniana da equação (5.1.40) pode ser escríto como sendo:

$$
H=-i \alpha_{1} \partial_{s}-i Z+\beta m
$$

onde $\alpha_{1}$ e $\beta$ estäo definidos respectivamente pelas equaçöes $(2.1 .4)$ e $(2.1 .6)$ e $Z$ está definido como sendo:

$$
Z=\left(\begin{array}{ll}
z & 0 \\
0 & z
\end{array}\right) \quad \text { e } \bar{z}=\left(\begin{array}{cc}
0 & \frac{\nu+1}{r} \\
-\frac{\nu}{r} & 0
\end{array}\right)
$$

O operador $\beta m$, como dissemos anteriormente, é obviamente simétrico, assim o problema é verificar se o operador:

$$
\bar{H}=-i \alpha_{1} \partial_{\mathrm{r}}-i Z
$$

ésimétrico.

Para essa finalidade calculemos, inicialmente:

$$
\begin{gathered}
\langle H \phi \mid \psi\rangle=\left\langle-i \alpha_{1} \partial_{r} \phi \mid \psi\right\rangle+\langle-i Z \phi \mid \psi\rangle \\
=\int d r r\left(-i \alpha_{1} \partial_{r} \phi\right\rangle^{\dagger} \psi+\int d r r(-i Z \phi)^{\dagger} \psi= \\
=\pi \int d r r \partial_{r} \phi^{\dagger} \alpha_{1} \psi+i \int d r r \phi^{\dagger}(Z)^{\dagger} \psi .
\end{gathered}
$$

Resolvendo a primeira integral da equação acima por partes, temos:

$$
\left\langle H_{\varphi} \phi \psi\right\rangle=i \int d r r \partial_{r}\left(\phi^{\dagger} \alpha_{1} \psi\right)-i \int d r r \phi^{\dagger} \alpha_{1} \partial_{r} \psi+i \int d r \phi^{\dagger}(Z)^{\dagger} \psi
$$


Novamente integrando por partes a primeira integral acima, temos:

$$
\langle\bar{H} \phi \mid \psi\rangle=i \int d r \partial_{r}\left(r \phi^{\dagger} \alpha_{1} \psi\right)-i \int d r \phi^{\dagger} \alpha_{1} \psi-i \int d r r \phi^{\dagger} \alpha_{1} \partial_{r} \psi+i \int d r r \phi^{\dagger}(Z)^{\dagger} \psi
$$

Calculemos agora o produto escalar $\langle\phi \mid \bar{H} \psi\rangle$ :

$$
\begin{gathered}
\langle\phi| H \psi=\left\langle\phi \mid\left(-i \alpha_{1} \partial_{r}-i Z\right) \psi\right\rangle \\
=-i \int d r r \phi^{\dagger} \alpha_{1} \partial_{r} \psi-i \int d r r \phi^{\dagger} Z \psi
\end{gathered}
$$

Voltando com os resultados das equaçōes (5.1.46) e (5.1.47), na imposicâa de operador simétrico dada pela equação (5.1.38), surge entäo a condição:

$$
\lim _{r \rightarrow 0} r\left(\phi^{\dagger} \alpha_{1} \psi\right)=0
$$

uma vez que as funço $\phi, \psi \in L^{2}(R)$ (ou seja, no limite de $r \rightarrow \infty$ essas funçoes tendem a zero) e uma vez que vale a ígualdade abaixo:

$$
-i \int d r \phi^{\dagger}\left(\alpha_{1}-r Z^{\dagger}\right) \psi=-i \int d r \phi^{\dagger}(r Z) \psi
$$

Lembrando da definição de $\phi$ dada pela equação (5.1.39), a equação (5.1.48) fornece:

$$
\begin{aligned}
& \lim _{r \rightarrow 0} r\left[\varphi_{+}^{(1)}+w_{11} \varphi_{-}^{(1)}+w_{12} \varphi_{-}^{(2)}\right]^{\dagger} \alpha_{1} \psi=0 \\
& \lim _{r \rightarrow 0} r\left[\varphi_{+}^{(2)}+w_{21} \varphi_{-}^{(1)}+w_{22} \varphi_{-}^{(2)}\right]^{\dagger} \alpha_{1} \psi=0
\end{aligned}
$$

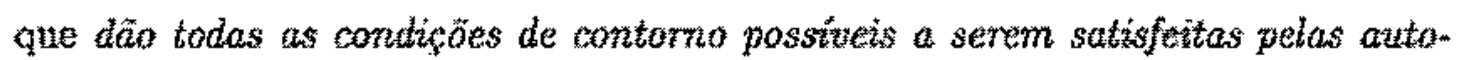
funçōes de $H^{4}$ na origem (na verdade dấo todas as condiçoes contono a serem satisfeitas por qualquer função no domínio do operutor $H$ ) e que correspondem também, exatamente, a todas as fanulias de quatro parämetros de extensões autoadjurtes possiveis para o operator $H$. 
De forma análoga, as condições de contorno admissíveis para o operador $H-\beta m$ podem ser parameltrizadas por uma matriz unitária $V$ da forma:

$$
V=\left(\begin{array}{ll}
\mathrm{v}_{11} & \mathrm{v}_{12} \\
\mathrm{v}_{21} & \mathrm{v}_{22}
\end{array}\right),
$$

através das equaçoes:

$$
\begin{aligned}
& \lim _{r \rightarrow 0} r\left[\chi_{+}^{(1)}+v_{11} \chi_{-}^{(1)}+v_{12} \chi_{-}^{(2)}\right]^{\dagger} \alpha_{1} \psi=0, \\
& \lim _{r \rightarrow 0} r\left[\chi_{+}^{(2)}+v_{21} \chi_{-}^{(1)}+v_{22} \chi_{-}^{(2)}\right]^{\dagger} \alpha_{1} \psi=0,
\end{aligned}
$$

uma vez que o operador $(H-\beta m)$ to o próprio operador $\bar{H}$ definido nas equaçós $(5.1 .41)$ e (5.1.42).

Mas lembrando que os operadores $\Lambda$ e $H-\beta m$ estão relacionados pela bansformaçäo unitária dada na equaçăo (2.1.18), as equaçōes acima, para as autofunçöes de A se tornàm:

$$
\begin{aligned}
& \lim _{t \rightarrow 0} r\left[U_{\chi}^{(3)}+v_{11} U \chi_{-}^{(1)}+v_{12} U_{\chi}^{(2)}\right]^{\dagger} \Sigma_{1} U \psi=0 \\
& \varliminf_{\tau \rightarrow 0} r\left[U_{\chi}^{(2)}+v_{21} U_{\chi}^{(1)}+v_{22} U_{\chi_{-}^{(2)}}^{(2)} \Sigma_{1} U \psi=0\right.
\end{aligned}
$$

onde usamos a unithiriedade da matriz $U$ :

$$
U U^{\dagger}=1 .
$$

Resumindo, podernos dizer que as exuações $(5.1 .50)$ e (5.1.51) dăo todas as extensöes auto-adjuntas de $H$ (parametrizadas por uma familia de quatro parârnetros) o que sirnultaneamente corresponde a todas as condiçoes de contorno admissiveis para as auto-funçốs de $H$. Do mesmo modo as equaçóes (5.1.55) e (5.1.56) dão todas as possiveis extensôes auto-adjuntas de $\Lambda$, isto 6 , todas as passíveis condicoes de contorno que as auto-fungoes de $\Lambda$ devem satisfazer na origem.

A tabela abaixo contem os resultados das exuacoies $(5.1 .50),(5.1 .51),(5.1 .55)$ e (5.1.56) e será útil nos próximos cálculos. 


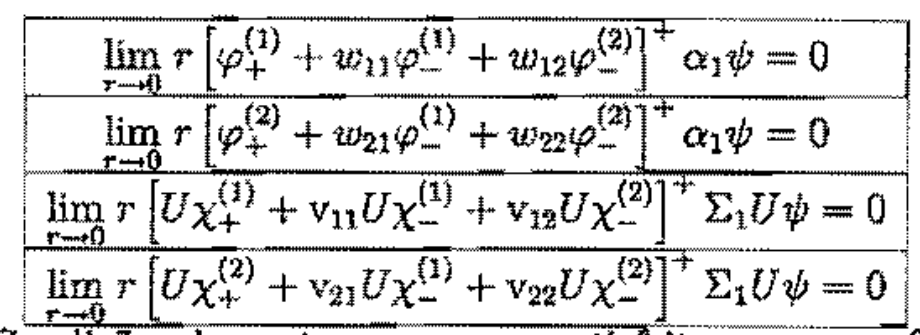

Tabela 1 - Condícōes de contomo a seren sátiffeitas por uma funça $\psi$ nos domínios mais gerais das extençoses auto adjuntas de $H$ (duas primeiras exuaçồes)

e de $\Lambda$ (duas últimas equaça 0 ).

\subsection{Extensöes Auto-Adjuntas para o Operador $H$ - Urn Caso Particular.}

Nessa seçäo vamos considerar uma classe especial de condiçöes de contorno com dois parametros para as auto-funçōes do operador hamiltoniana que inclui as anteriormente propostas na literatura [1], 2$],[3],[4],[5],[6]$ e [7].

Em seruida, na próxima seção, vamos impor suto-funções do operador helicidade $\Lambda$ as mesma condiçöes de contorno impostas às auto-funçoes do operador haruiltoniana $H$. De forma que estaremos assim definindo os dois operadores em domínios comuns, isto e $D(H)=D(\Lambda)$. Levando em conta que os dois operadores $H$ e $\Lambda$ formalmente comutam, estudaremos mais adiante, a possibilidade de se obter auto-fungoes comuns e se as dinâmicas obtidas para $H$ de fato preservam a halicidade e simetria $\phi \rightarrow \phi+1$ do hamiltoniana.

Vanos agora tomar a matriz paramétrica $W$ da equação (5.1.37) camo tendo a forma diagonal particular da referência [1], isto ë:

$$
W=\left(\begin{array}{cc}
e^{i w_{1}} & 0 \\
0 & e^{i t w_{2}}
\end{array}\right)
$$

Assim as relacoes dadas pelas equaçöes $(5.1 .50)$ e (5.1.51) (arresentadas na Tabela 1) ficam:

$$
\begin{aligned}
& \lim _{r \rightarrow 0} r\left[\varphi_{+}^{(1)}+e^{i \omega i_{1}} \varphi_{-}^{(1)}\right]^{\dagger} \alpha_{1} \psi=0 \\
& \lim _{r \rightarrow(0)}\left[\varphi_{+}^{(2)}+e^{i \omega_{2}} \varphi_{-}^{(2)}\right]^{\dagger} \alpha_{1} \psi=0
\end{aligned}
$$


onde $\psi$ um uspinor de quatro componente que cá no domínio da extensäo anto-adjunta de $H$ :

$$
\psi=\left(\begin{array}{l}
w_{1} \\
\psi_{2} \\
\psi_{3} \\
\psi_{4}
\end{array}\right)
$$

Substituindo o restultado da equação $(5.1 .19)$ na equação $(5.2 .2)$ ternos:

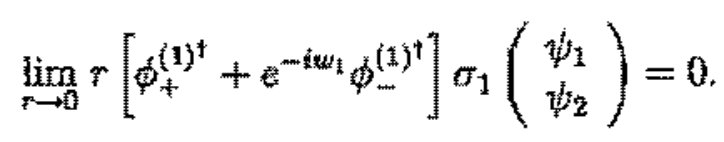

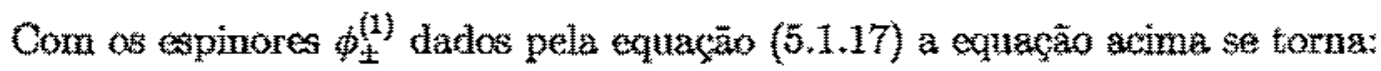

$$
\lim _{r \rightarrow 0} r\left(\left(e^{-i \frac{\pi}{4}}-e^{-i \mu_{1}+i \frac{x}{4}}\right) K_{\psi+1}(\sqrt{2} m r) \psi_{1}+\left(1+e^{-i w_{1}}\right) K_{1}(\sqrt{2} m r) \psi_{2}\right)=0
$$

Lembrando do comportamento assintótico de $K_{\nu}(x)$ e de $K_{y+1,1}[10]_{\text {: }}$

$$
\begin{gathered}
K_{\nu}(x) \sim \frac{1}{2} \Gamma(-\nu)\left(\frac{1}{2} x\right)^{-\nu} \\
K_{y+1}(x) \sim \frac{1}{2} \Gamma(1+\nu)\left(\frac{1}{2} x\right)^{-(1+x)}
\end{gathered}
$$

e introduzindo as definigồes:

$$
\begin{gathered}
x=\frac{1}{2} \Gamma(1+\nu)\left(\frac{m}{\sqrt{2}}\right)^{-(1+\nu)} \lim _{r \rightarrow+4} r^{-\nu \psi_{1}} \psi_{1} \\
y=\frac{1}{2} \Gamma(-\nu)\left(\frac{m}{\sqrt{2}}\right)^{\nu} \lim _{r \rightarrow 0} r^{(1+\nu)} \psi_{2}
\end{gathered}
$$

temos que a equaçäo (5.2.5) acima se toma:

$$
\left[e^{-i \frac{1}{4}}-e^{-i(t)+i \frac{\pi}{i}}\right] x-i\left[i\left(1+e^{-i w_{1}}\right)\right] y=0 .
$$


Podemos escrever a equaçāo (5.2.9) assim:

$$
\cos \delta_{1} x-i \operatorname{sen} \delta_{1} y=0
$$

onde:

$$
\operatorname{tg} \delta_{1}=\frac{\sqrt{2}}{\operatorname{tg}\left(\frac{w_{1}}{2}\right)-1}
$$

que corresponde exatamente a classe de condiçóes de contorno discutidas por Sousa Gerbert (veja referência [3]), onde foram obtidas todas as extensöes autoadjuntas a um parâmetro para o problema de uma partícula sem spin em duas dirnensöes $[1,3]$.

Da mesma forma, substituindo agora, os resultados da equação (5.1.20) na condição dada pela equação (5.2.3) temos:

$$
\lim _{r \rightarrow 0} r\left[\hat{\phi}_{+}^{(2)^{\dagger}}+e^{-i+w_{2}} \phi_{-}^{(2)^{\dagger}}\right] \sigma_{1}\left(\begin{array}{c}
\psi_{3} \\
\psi_{4}
\end{array}\right)=0
$$

Com as espinores $\phi^{(2)}$ datos pela equaçäo (5.1.18) a equaçào (5.2.13) acima se torna:

$$
\lim _{r \rightarrow 0}\left[\left(e^{i \frac{\pi}{4}}-e^{-i t w_{2}-i \frac{\pi}{4}}\right) K_{t+1}(\sqrt{2} m r) \psi_{3}+\left(1+e^{-i w w_{2}}\right) K_{y}(\sqrt{2} m r) \psi_{4}\right]=0
$$

Usando novamente as forruas assintóticas de $K_{y}$ e $K_{x+1}$ dadas pela equação (5.2.6) e introduzindo as definiçoes:

$$
\begin{gathered}
z=\frac{1}{2} \Gamma(1+\nu)\left(\frac{m}{\sqrt{2}}\right)^{-(1+\nu)} \lim _{r \rightarrow 0} r^{-v} \psi_{3}, \\
w=\frac{1}{2} \Gamma(-\nu)\left(\frac{m}{\sqrt{2}}\right)^{\nu} \lim _{r \rightarrow 0} r^{(1+\nu)} \psi_{4},
\end{gathered}
$$


a equagà̃o $(5.2 .14)$ se torna:

$$
\cos \hat{\delta}_{2} z-i \operatorname{sen} \delta_{2} z=0
$$

onde:

$$
\operatorname{tg} \delta_{2}=\frac{\sqrt{2}}{\operatorname{tg}\left(\frac{\operatorname{tg}}{2}\right)+1}
$$

Assim, a partir das equaçōes (5.2.11) e (5.2.17) concluímos que as condições de contorno a serem satisfeits pelas auto-funçö de $H$ säo:

$$
\begin{aligned}
& x \cos \delta_{1}-\operatorname{sinsen} \delta_{1}=0 \\
& z \cos \delta_{2}-\operatorname{susen} \delta_{2}=0
\end{aligned}
$$

que significam condiçōes a serem satisfeitas separadamente pelas componentes superior $(s=+1)$, exuaça $(5.2 .19)$, e inferior $(s=-1)$, equaçào $(5.2 .20)$, dos espinores de quatro componentes das auto-funçoes de $H$. Em outras palavras, as condições de contorno dadas acima não acoplan as componentes superior e inferior das auto-funcoos de $H$.

\subsection{As condiçōes de contorno maís gerais admissíveis para o operador A.}

Vamos agora encontrar condiçös de contorno mais gerais a serem satisfeitas pelas anto-funçôes do operador helicidade, com a "matriz paramétrica " $V$ dada pela equaçăo (5.1.52). Para encontrar tais condigöes de contorno devenos retormar às equagoes $(5.1 .55)$ e (5.1.56).

Da equaciano $(5.1 .55)$ temos:

$$
\lim _{r \rightarrow 0} r\left[U \chi_{+}^{(1)}+v_{1 \lambda} U X_{-}^{(1)}+v_{12} U \chi\right]^{\dagger} \Sigma_{14}+\frac{1}{4}=0
$$

com $\psi$ sendo uma função qualquer no domínio maks geral onde o operador helicidade é auto-adjunto $(D(\Lambda))$ a ser determinado:

$$
\psi \in D(\Lambda)
$$

Lembrando da forma da matriz unitária $U$ dada pela equação (2.1.18) e das formas dos espinores $\chi_{ \pm}^{(1)}$ e $\chi_{ \pm}^{(2)}$ dadas pela equacă (5.1.3.3), podemos escrever a 
expressão entre colchetes do espinor da equacão (5.3.1) acima como sendo igual a:

$$
\begin{gathered}
U X_{+}^{(1)}+v_{11} U \chi_{-}^{(1)}+v_{12} U X_{-}^{(2)}= \\
\frac{1}{\sqrt{2}}\left[\begin{array}{cc}
I & -i \sigma_{3} \\
I & i \sigma_{3}
\end{array}\right]\left[\begin{array}{c}
\phi_{+} \\
0
\end{array}\right]+v_{11} \frac{1}{\sqrt{2}}\left[\begin{array}{cc}
I & -i \sigma_{3} \\
I & \hat{w}_{3}
\end{array}\right]\left[\begin{array}{c}
\phi_{-} \\
0
\end{array}\right]+v_{12} \frac{1}{\sqrt{2}}\left[\begin{array}{cc}
I & -i \sigma_{3} \\
I & i \sigma_{3}
\end{array}\right]\left[\begin{array}{c}
0 \\
\phi_{-}
\end{array}\right]= \\
\frac{1}{\sqrt{2}}\left[\begin{array}{c}
\phi_{+} \\
\phi_{+}
\end{array}\right]+\frac{1}{\sqrt{2}} v_{11}\left[\begin{array}{c}
\phi_{-} \\
\phi_{-}
\end{array}\right]+\frac{1}{\sqrt{2}} v_{12}\left[\begin{array}{c}
-i \sigma_{3} \phi_{-} \\
i \sigma_{3} \phi_{-}
\end{array}\right]
\end{gathered}
$$

Lembrando das formas do ds dadas através da tuagäo (5.1.34), vemos que:

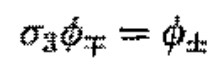

De forma que a equagäo (5.3.3) se torna:

$$
\frac{1}{\sqrt{2}}\left[\begin{array}{c}
\left(1-\hat{n} v_{12}\right) \phi_{+}+v_{11} \phi_{-} \\
\left(1+i \nu_{12}\right) \phi_{+}+v_{11} \phi_{-}
\end{array}\right] .
$$

Assim a equagăo (5.3.2) com a substituiçăo do restltado de (5.3.5) pode ser escrita como:

$$
\begin{aligned}
& \lim _{r \rightarrow 0} \frac{1}{\sqrt{2}} r\left[\left(1-w_{12}\right) \phi_{+}^{\dagger}+w_{11} \phi_{-}^{\dagger}\right] \sigma_{1}\left(\begin{array}{l}
\psi_{1} \\
\psi_{2}
\end{array}\right)+ \\
& +\lim _{r \rightarrow 0} \frac{1}{\sqrt{2}} r\left[\left(1+\bar{w}_{12}\right) \phi_{+}^{\dagger}+\bar{v}_{11} \phi_{-}^{\dagger}\right] \sigma_{1}\left(\begin{array}{l}
\psi_{3} \\
\psi_{4}
\end{array}\right)=0
\end{aligned}
$$

Lembrando das formas de $\phi_{z}$ dadas através da equaça (5.1.34), dos comportamentos assintotticos de $K_{*}$ e $K_{t+1}$ dado pela equagäo (5.2.6) e usando as definicones para $x$ e y dadas $\operatorname{com}(5.2 .7)$ e $(0,2.8)$ e usando as definiçöes para $z$ e $w$ dadas em $(5.2 .15)$ e $(5.2 .16)$ apenas tomando $l=\sqrt{2} m$ nessas definições, a equação (5.3.6) acima se toma:

$$
\left(1-\bar{v}_{11}-\bar{v}_{12}\right) x+\left(1+\bar{v}_{11}-\bar{v}_{12}\right) y+\left(1-\bar{v}_{11}+i \bar{v}_{12}\right) z+\left(1+\bar{v}_{11}+i \bar{v}_{12}\right) w=0
$$

onde a barra sobre os parâmetros v's denota o complexo conjugado. Esta $b$ a primeira equação para as condiçöes de contorno mais gerais para a helicidade. 
Da equaçäo (5.1.56), temos:

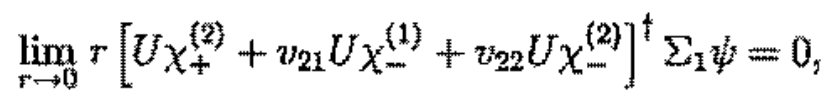

com w sendo uma funça no dominio de $\Lambda$ a ser determinado:

$$
\psi \in D(h)
$$

Procedendo da mesma forma que levou a exuacă $(5.3 .1)$ a poder sen escrita como a equasiâ (5.3.7), podemos reescrever a eq. (5.3.8) como sendo:

$$
\left(i-\bar{v}_{21}-i \bar{v}_{22}\right) z+\left(-i+\bar{v}_{21}-\bar{z}_{22}\right) y+\left(-i-\bar{v}_{21}+\bar{v}_{22}\right) z+\left(i+\bar{v}_{21}+i \bar{v}_{22}\right) w=0 .
$$

Assim as condiçồ de contorno mais gerais a serem satisfeitas pelas autofuncōes da helicidade parametrizadas pela matriz $V$ unitária säo:

$$
\left(1+\bar{v}_{11}+\bar{c}_{12}\right) w+\left(1-\bar{v}_{11}+i \bar{v}_{12}\right) z+\left(1+\bar{v}_{11}-i \bar{v}_{12}\right) y+\left(1-\bar{v}_{11}-i \bar{v}_{12}\right) x=0
$$

$$
\left(i+\bar{v}_{21}+i \bar{v}_{22}\right) w+\left(-i-\bar{v}_{21}+i \bar{v}_{22}\right) z+\left(-i+v_{21}-\bar{v}_{2 z_{2}}\right) y+\left(i-v_{21}-i \bar{v}_{22}\right) x=0
$$

as quais, evidentemente, por serem as mais gerais, acoplam todas as componentes dos espinores de quatro componentes das auto-funçöes de $\Lambda$.

Se quisermos definir o dominio do operador $\Lambda$, dado através das equações (5.3.11) e (5.3.12) acima como sendo o mesmo domínio do operador $H$, definido através dns equacöes (5.2.19) e (5.2.20), ou seju, se quisernos impor as mesmas

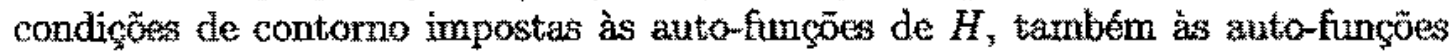
de $A$, devemos impor que o sistema de equações (5.2.19) e (5.2.20) e o sistema de equaçöes (5.3.11) e (5.3.12) tenham o mesmo conjunto de soluçotes, ou seja, sejam equivalentes. Para encontrarmos as condiço necessúrias e suficientes para que isso aconteça, basta começarmos mulaplicando a equaça (5.3.11) por $\cos \delta_{1} \cos \delta_{2}$ e entä̀ substiturmos as equaços $(5.2 .19)$ e (5.2.20). 
Assim obtemos as condiçoes:

$$
\begin{aligned}
& e^{i w_{2}} v_{11}-i e^{-i \hat{\delta}_{2}} v_{12}=-e^{-i \dot{t}_{2}} \\
& e^{i \delta_{1}} v_{11}+i e^{-i \delta_{1}} v_{12}=-e^{-i \delta_{k}} .
\end{aligned}
$$

Multiplicando (5.3.12) por $\cos \delta_{1} \cos \delta_{2}$ em seguida substituindo as equaçö $(5.2 .19)$ e $(5.2 .20)$ encontramos as condiços:

$$
\begin{aligned}
& -i \mathrm{e}^{-i \tilde{f}_{2} v_{22}}+e^{i \delta_{2}} v_{21}=i e^{i \delta_{3}} \\
& 3 e^{-i \delta_{1}} q_{22}+e^{i \delta_{1}} \psi_{21}=-i e^{i \sigma_{1}}
\end{aligned}
$$

Temos, assim dois sistemas de equaçó desacoplados, que terão soluçäo apenas se:

$$
\cos \left(\delta_{1}-\delta_{2}\right) \neq 0
$$

Sob essa condiçăo podemos resolver o sistema definido pelas equaçōes (a.3.13) e (5.3.14), obtendo como únicas soluçöes:

$$
\begin{gathered}
v_{11}=\frac{-2}{e^{2 i \delta_{1}}+e^{2 i \delta_{2}}} \\
v_{12}=-i \frac{\left(e^{-2 i \delta_{2}}-e^{-2 \delta_{1}}\right)}{e^{-2 i_{2}}+e^{-2 i \delta_{1}}}
\end{gathered}
$$

O sistema definido pelas exuagoes $(5.3 .15)$ e (5.3.16) fornece, sob a condicäo (5.3.17), as soluçôes úmicas:

$$
\begin{aligned}
& v_{21}=i \frac{\left(e^{2 i \delta_{2}}-e^{2 i \delta_{1}}\right)}{e^{2 i \delta_{1}}+e^{2 i \delta_{2}}} \\
& v_{22}=\frac{-2}{e^{2 i^{2} \delta_{1}}+e^{-2 i \delta_{2}}} .
\end{aligned}
$$

De onde vemos que:

$$
v_{11}=\overline{v_{22}} \text { e } \quad v_{12}=\overline{v_{21}} \text {. }
$$


No entanto, a matriz $\mathrm{V}$ deve ser unitáriá, isto é:

$$
V V^{*}=1
$$

Assim:

$$
\begin{aligned}
& \left(\begin{array}{ll}
v_{11} & v_{12} \\
v_{21} & v_{22}
\end{array}\right)\left(\begin{array}{ll}
\overline{v_{11}} & \overline{v_{21}} \\
\overline{v_{12}} & \overline{v_{22}}
\end{array}\right)=\left(\begin{array}{ll}
1 & 0 \\
0 & 1
\end{array}\right) \\
& \left(\begin{array}{ll}
v_{11} \overline{v_{11}}+v_{12} \overline{w_{12}} & v_{11} \overline{w_{21}}+v_{12} \overline{z_{22}} \\
v_{21} \overline{v_{11}}+v_{22} \overline{v_{12}} & v_{21} \overline{v_{21}}+v_{2 z} \overline{v_{22}}
\end{array}\right)=\left(\begin{array}{ll}
1 & 0 \\
0 & 1
\end{array}\right)
\end{aligned}
$$

Devido às relaçöes expressas pela equaçảo (5.3.22), é fácil notar na equaçāo acima que os elementos da diagonal prinncipal säo idênticos, bem como os elementos fora da diagonal tambérxı sâo idênticos entre si.

Assim basta verificar que:

$$
v_{11} u_{92}+v_{12} v_{21}=1
$$

6

$$
v_{11} v_{12}+v_{12} v_{11}=2 v_{11} v_{12}=0 .
$$

A equtação (5.3.25) conduz à condição:

$$
\cos \left(\delta_{2}-\delta_{1}\right)=1
$$

A equaçăo $(5.2 .26)$ condua à equaçäo:

$$
\operatorname{sen}\left(\delta_{1}-\delta_{2}\right)=0 .
$$

Portanto, a condiçäo de unitariedade da matriz paramétrica $Y$ conduz finalmente à condiçăo:

$$
\delta_{1}-\hat{\delta}_{2}=\overline{2} n \pi \quad, \quad n \in Z .
$$

A equaçăo acima deve ser a condiçâo necessária e suficíente para que as condiçöes de contorno parametrizadas por $\delta_{1}$ e $\delta_{2}$ dadas pelas equaçöes $(5.2 .19)$ e (5.2.20) sejam tamberm admissiveis para a helicidade.

Note que a condiçâ (5.3.29) implica em mesmas condiçöes de contorno a serem satisfeitas pelas componentes superior e inferior dos espinores de quatro 
componentes das auto hunçôs de $H$ (devido a periodicidade das funçôs seno cosseno das equaço $(5.2 .19)$ e $(5.2 .20))$.

Note tambem que as auto-fungôes assim obtidas (isto é, que satisfarem às condiçōes de contorno dadas por $(5.2 .19)$ e (5.2.20) com a condição (5.3.39) satisfeita) incluem as apresentadas nas referencias [3], [4] te nas referências [1], [2]. Isso ficará mais evidente ao escrevermos explicitamente as auto-funções de $H$ que obedexem às equaçós $(5.2 .19)$ e $(5.2 .20)$.

Um modo particular de satisfazer a equaçäo (5.3.29) é tomando:

$$
\delta_{1}=\delta_{2}=\delta
$$

Nessa situaçăo as equaçó $(5.3 .18),(5.3 .19),(5.3 .20)$ e $(5.3 .21)$ se tornam:

$$
v_{11}=-e^{-2 i \delta} \quad v_{22}=\overline{v_{11}}=-e^{2 i \delta} \quad v_{12}=\overline{v_{21}}=0
$$

De modo que as condições de contorno dadas por $(5,3,11)$ e $(5.3 .12)$ ficam:

$$
\begin{aligned}
& \cos \delta(x+z)-i \operatorname{sen} \delta(y+z)=0 \\
& \cos \delta(x-z)-i \operatorname{sen} \delta(y-w)=0
\end{aligned}
$$

O sistema de equaçoes acima, evitentemente, é equivalente ao sistema:

$$
\begin{aligned}
& x \cos \delta-i y \operatorname{sen} \delta=0 \\
& z \cos \delta-i \omega 2 \operatorname{sen} \delta=0 .
\end{aligned}
$$

Assim, vemos que, de fato, foi operacionalizado através das equaços (5.3.18) a (5.3.21) junto com as equaços $(5.3 .29)$ e (5.3.30), o estabelecirnento de mesmas condiçô de contorno a serem satisfeitas tanto pelas auto-funçöes de $I$ quanto pelas auto-funcöes de $A$, o que significa a determinagäo de domínios $D_{\delta}(H, A)$ comuns aos operadores em questão; isto é.

$$
D_{6}\left(H_{3} \Lambda\right)=D_{\delta}(H)=D_{\delta}(\Lambda) \text {. }
$$

Vamos, entäo, resolver o problema de determinaçẩ das auto-funços de $H$ e $\Lambda$ que satisfarem as condiçōes de contorno acima, respectivarnente nas $\$$ exôt 5.5 e 5.6 . 


\subsection{Condiçôs de Contorno e Conservação da Helicidade.}

Tendo definido, na sesca anterior, os operadores $H$ e $A$ atuando em domínios den* sos comuns $D_{\delta}(H, \Lambda)$ e levando em conta que esses dois operadores formalmente comutam, isto $\hat{\text { ét }}$

$$
[H, \Lambda]=0
$$

queremos estudar a possibilidade de obtençäo de autorfungöes comuns aos dois operadores e se as dinâmicas obtidas para $H$ atravtés das condiçôes de contorno dadas pelas equagötes $(5.2 .19)$ e $(5.2 .20)$ conservam a helicidade. Faremos esse estudo nas Seçôtes 5.7 a 5.10 .

Essa questëo foi, primeiramente, levantada por Hagen [7] e posteriormente abordada pelos autores Coutinho e Perez [1]. Essa questão será reestudada nas próximas secón, levando a condusốes, a principio, diferentes das obtidas por esses autores.

Antes de prosseguirmos, no entanto, vamos recupitular alguns de nossos principais resultados.

As condiçöes de contorno a serem satisfeitas pelas auto-funções de $H$ e $A$ parametrizadas pelo parâmetro $\delta$ e que definem os domínios commons $D_{6}(H, A)$ onde $H$ e $A$ säo auto-adjuntos (encontradas na seçäo anterior) sāo:

$$
\begin{aligned}
& x \cos \delta-y b \operatorname{sen} b=0 \\
& z \cos \delta-2 x w \operatorname{sen} \delta=0
\end{aligned}
$$

onde:

$$
\begin{aligned}
& x=\frac{1}{2} \Gamma(1+u)\left(\frac{n}{\sqrt{2}}\right)^{-(1+\nu)} \lim _{r \rightarrow 0} r^{-\psi} \psi_{1}(r), \\
& y=\frac{1}{2} \Gamma(-v)\left(\frac{m}{\sqrt{2}}\right)^{n} \lim _{r \rightarrow 0} r^{(1+y)} \psi_{2}(r) \\
& z=\frac{1}{2} \Gamma(1+\nu)\left(\frac{m}{\sqrt{2}}\right)^{-(1+\nu)} \lim _{r \rightarrow 0} r^{-\nu} \psi_{3}(r), \\
& w=\frac{1}{2} r(-n)\left(\frac{m}{\sqrt{2}}\right)^{*} \lim _{r \rightarrow 4} r^{(1+n)} \psi_{4}(r)
\end{aligned}
$$


Vamos resolver respectivamente nas Seçōes 5.5 e 5.6 o problema da determinação da auto-funçōes dos operadores $H$ e $\Lambda$ que satisfazem às condiçōes de contorno dadas pelas equaçōes acima (5.4.2) e (5.4.3).

\subsection{Determinaçào das auto-funçōes do operador $H$ que satisfazem as condições de contorno desacopladas.}

No Capítulo 2, já resolvemos o problema da determinação das auto-funçōes mais gerais de $H$. As auto-funções de $H$ encontradas naquele capítulo, dadas pela equação (2.2.31), são:

$$
\psi_{ \pm}(k r)=\left(\begin{array}{c}
\alpha \phi_{ \pm}^{(1)}(k r) \\
\beta \phi_{ \pm}^{(2)}(k r)
\end{array}\right)
$$

onde $\alpha$ e $\beta$ são constantes quaisquer que regulam as proporçöes entre as componentes superior e inferior, que são dadas pelas equações (2.2.29) e (2.2.30) respectivamente, abaixo transcritas para maior comodidade do leitor:

$$
\begin{aligned}
& \phi_{+}^{(1)}(k r)=\left(\begin{array}{c}
J_{|\nu|}(k r)+c_{+} J_{-|v|}(k r) \\
\frac{-i k}{|E|+m}\left(J_{|\nu|-1}(k r)-c_{+} J_{-|\nu|+1}(k r)\right)
\end{array}\right), \\
& \phi_{+}^{(2)}(k r)=\left(\begin{array}{c}
J_{|\nu|}(k r)+d_{+} J_{-|\nu|}(k r) \\
\frac{-i k}{|E|-m}\left(J_{|\nu|-1}(k r)-d_{+} J_{-|\nu|+1}(k r)\right)
\end{array}\right), \\
& \phi_{-}^{(1)}(k r)=\left(\begin{array}{c}
J_{|\nu|}(k r)+c_{-} J_{-|v|}(k r) \\
\frac{i k}{|E|-m}\left(J_{|\nu|-1}(k r)-c_{-} J_{-|\nu|+1}(k r)\right)
\end{array}\right), \\
& \phi_{-}^{(2)}(k r)=\left(\begin{array}{c}
J_{|\nu|}(k r)+d_{-} J_{-|\nu|}(k r) \\
\frac{i k}{|E|+m}\left(J_{|\nu|-1}(k r)-d_{-} J_{-|\nu|+1}(k r)\right)
\end{array}\right) .
\end{aligned}
$$

Vamos impor a essas auto-funções mais gerais que satisfaçam às condições de contorno dadas por (5.4.2) e (5.4.3) que desacoplam as componentes superior e inferior dos espinores $\psi_{ \pm}(k r)$ das auto-funçōes de $H$. Para tanto devemos calcular $x, y, z$ e $w$ para cada um dos espinores $\psi_{ \pm}(k r)$, lembrando do comportamento na origem das funções de Bessel envolvidas.

Lembrando do comportamento na origem das funções de Bessel ${ }^{[10]}$ :

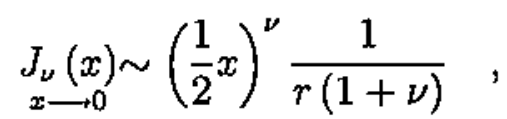


e lembrando que estamos tratando do caso em que $-1<\nu<0$, temos que, para a auto-função $\psi_{+}(k r)$ dada pela eq. (5.5.1) acima:

$$
x=\alpha(\sqrt{2} m)^{\{v \mid-1} k^{-|v|} c_{+},
$$

uma vez que $J_{[\nu \mid}(k r)$ tem comportamento regular na origem e $J_{-|\nu|}(k r)$ é singular na origem, e :

$$
y=\alpha \frac{-i(\sqrt{2} m)^{-|\nu|}}{|E|+m} k^{|v|},
$$

uma vez que $J_{|\nu|-1}$ é singular e $J_{-|\nu|+1}$ é regular na origem.

Substituindo as expressōes de $x$ e $y$ acima obtidas na condição de contorno (5.4.2), vem que:

$$
c_{+}(\delta, E)=\frac{\operatorname{tg} \delta}{|E|+m} \frac{k^{2|v|}}{(\sqrt{2} m)^{2|v|-1}} .
$$

Procedendo da mesma forma, obtemos as demais constantes $d_{+}, c_{-}$e $d_{-}$como funçōes do parâmetro $\delta$ e da energia. Os resultados estão apresentados na tabela abaixo:

$$
\begin{array}{|l|l|}
\hline c_{+}=\frac{t g \delta}{|E|+m} \frac{k^{2|v|}}{(\sqrt{2} m)^{2|v|-1}} & c_{-}=-\frac{\operatorname{tg} \delta}{|E|-m} \frac{k^{2|v|}}{(\sqrt{2} m)^{2|v|-1}} \\
\hline d_{+}=\frac{l g \delta}{|E|-m} \frac{k^{2}|v|}{(\sqrt{2} m)^{2|v|-1}} & d_{-}=-\frac{t g \delta}{|E|+m} \frac{k^{2|v|}}{(\sqrt{2} m)^{2|v|-1}} \\
\hline
\end{array}
$$

Tabela 2 - Valores das constantes $c_{ \pm}$e $d_{ \pm}$em função do parâmetro $\delta$ das extensões auto- adjuntas.

Note que esses resultados coincidem com os resultados obtidos na referência [3] do autor Sousa Gerbert.

Assim, as auto-funções de $H$ que satisfazem às condiçōes de contorno desacopladas das equações (5.4.2) e (5.4.3) tem a forma dada pelas equações (5.5.1) a (5.5.5) com as constantes $c_{ \pm}$e $d_{ \pm}$dadas na Tabela 2. 
5.6. Determinação das auto-funçoes do operador $\mathbf{A}$ que satisfazem as condiçōes de contorno desacopladas.

Vamos agora passar ao problema da teterminaça das auto-funçoes de $A$ que satisfazem às mesmas condiçoes de contorno desacopladas (5.4.2) e (5.4.3).

As auto-funçōes mais gerais de $\Lambda$ já foram encontradas no Capítulo 2, através das equações $(2.3 .17)$ e $(2.3 .18)$ e são as seguintes:

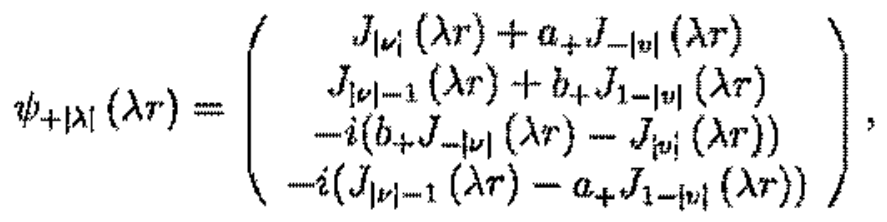

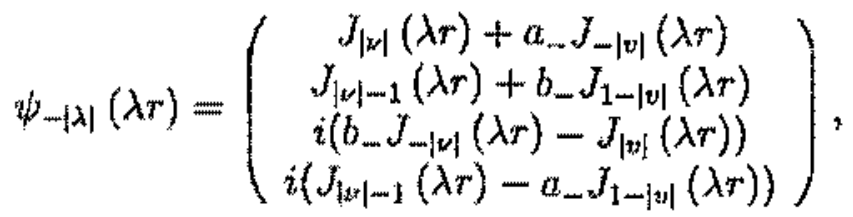

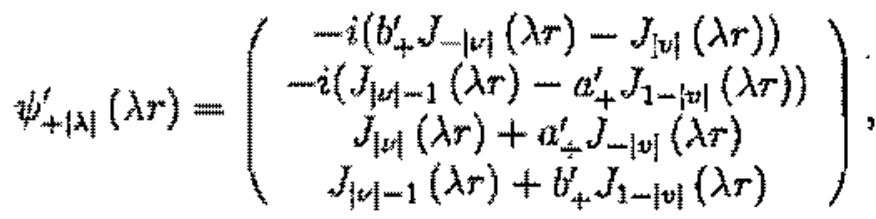

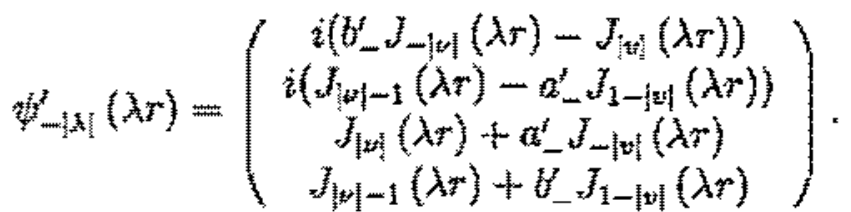

Devernos impor a essos auto-funço mais gerais que satisfacam às condicoes de contorno desacopladas $(0.4,2)$ e $(5,4,3)$. Para tanto devemos calcular $w_{3}, z$ e w para cada um dos quatro espinores auto-funcōes de $\Lambda$, através das definicôt $(5.4 .4)$ a $(5.4 .7)$ tomando $l=\sqrt{2} m$ nessas definiços. Dado o comportamento das funço de Bessel na origem (equacaro $(5.5 .6)$ ) para o espinor $\psi_{+|x|}(\mathrm{Ar})$, temos

$$
x=b^{|y|-1} \lambda^{-|p|} a
$$


uma vez que $J_{w}(\lambda r)$ é regular $\mathrm{e} J_{\text {mol }}(\lambda r)$ es singular na origem e:

$$
y=t^{-m \mid} \lambda^{\mid x-1}
$$

uma vez que $J_{i p \mid-1}(\lambda r)$ e singular e $J_{-[p \mid+1}(\lambda r)$ é regular na origern.

Substituindo a y obtidos acima na condiçăo dada por $(5,4,2)$ obtemos:

$$
a(\delta, \lambda)=\left(\frac{\lambda}{l}\right)^{2|x|-1} \operatorname{tg} \delta
$$

A imposição da condigäa $(5.4 .3)$ à mesma auto-funçăo $\psi_{+1 \lambda}(\lambda r)$ também leva ao mesmo resultado de (5.6.7) para o parâmetro $b_{+}$.

Procedendo da mesma forma com as demais atto-funçöes, obtemos as demais constante $a_{\ldots}, b_{\ldots}, a_{ \pm}^{\prime}$ e $b_{+}^{\prime}$. Os resultados estäo apresentados na tabela abaixo:

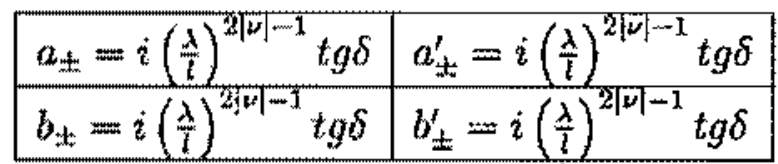

Tabela 3 - Valores das constantes $a_{ \pm}^{\left.()^{\prime}\right)}$ e $b_{ \pm}^{(\prime)}$ em funçäo do parâmetro $\delta$ das extensöes auto- adjuntas.

Assirn, as auto-funçốes de $A$ que satisfazem às condiçôes de contorno desacopladas tem a forma dada pelas equaçós (5.6.1) a (5.6.4) com as constantes a the dadas na Tabela 3.

\subsection{Discussäo dos Resultados.}

Tendo definido os operatores $H$ e atuando no mesmo dominio $D_{5}(H, \Lambda)$, definidos atraves das condiçoes de contomo desacopladas $(5.4 .2)$ e $(5.4 .3)$, e tendo em vista que estes operadores comutam formalmente, poderiamos esperar que esses fatos, por si so, talvez implicassem que $H$ e $A$ comutassem efetivamente no domínio definido $D_{6}(H, \Lambda)$, e que conseduentemente tivessem auto-fungöos cormuns nesse

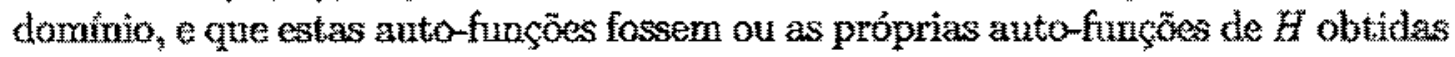
na Segăo 5.5, ou as proprias auto-funções de $A$ obtidas na Secāo 5.6 ou alguma combinaga linear simples dessas auto-funcôes. Este "raciocinio natural " esta sugerido na referência [y] e conforme verificaremos a seguir nâo correto.

Na verdade, conforme discutiremos mais adiante (Seçâ 5.10$)$, o operador $A$ ao atuar nas auto-funçōes de $H$ encontradas na Seçäo 5.5 , năo gera vetores pertencentes ao domínio $D_{\varepsilon}(H, A)$ para todos os valores de $\bar{c}$, ou seja, gera, para 
certas axtensöes, vetores fora desse domínio. Em outras palavras, o operador $A$ nüo deixa o dominio $D_{\delta}(H, A)$ invariante.

Nesse caso, näo faz sequer sentido pensamos em uma relaçăo de comutaçäo entre $H$ e $\Lambda$ em $D_{b}(H, \Lambda)$ e consequentemente năo faz sentido falarmos em auto funçöes simultâneas.

Isso é urn fato inesperado. Mas nós nâo levaremos discussäo adictute agora, sem primeiro tentar responder à seguinte pergunta:

- "Qual a condiçäo para gue os oparadones $H$ e A tentam auto-funcöes com matns?"

5.8. Determinaçäo da condiçäo formal para que os operadores $H$ e $A$ tenham auto-funçöes comuns.

Vamos agora procurar sob que condiçöes existe alguma combinação linear de autofungoes $\psi_{\text {t }}$ de $H$, dadas através das constantes $\alpha$ e $\beta$ da equacăo $(5.5 .1)$, que seja tambérm auto-funçäo de $A$ definido pela equacâo (2.3.3).

Assim, vamos impor:

$$
\begin{gathered}
\Lambda \psi_{ \pm}(k r)=\lambda \psi_{ \pm}(k r) \\
\left(\begin{array}{ll}
0 & h \\
h & 0
\end{array}\right)\left(\begin{array}{ll}
\alpha & \phi_{ \pm}^{(1)}(k r) \\
\beta & \phi_{ \pm}^{0}(k r)
\end{array}\right)=\lambda\left(\begin{array}{cc}
\alpha & \phi_{ \pm}^{(1)}(k r) \\
\rho & \phi_{ \pm}^{(2)}(k r)
\end{array}\right) \\
\left(\begin{array}{ll}
\rho n & \phi_{t}^{(2)}(k r) \\
\alpha h & \phi_{ \pm}^{(1)}(k r)
\end{array}\right)=\lambda\left(\begin{array}{cc}
\alpha & \phi_{ \pm}^{(1)}(k r) \\
\beta & \phi_{ \pm}^{(2)}(k r)
\end{array}\right)
\end{gathered}
$$

Usando a equaça $(2.2 .18)$, temos:

$$
\begin{aligned}
& \left(h+\sigma_{3} m\right) \phi_{ \pm}^{(1)}(k r)= \pm|E| \phi_{ \pm}^{(1)}(k r) \\
& \left(h-\sigma_{3} m\right) \phi_{ \pm}^{(2)}(k r)= \pm|E| \phi_{ \pm}^{(2)}(k r)
\end{aligned}
$$

de modo que:

$$
\begin{aligned}
& h \phi_{ \pm}^{(1)}(k r)=\left( \pm|E|-\sigma_{3} m\right) \phi^{(1)}(k r) \\
& h \phi_{ \pm}^{(2)}(k r)=\left( \pm|E|+\sigma_{3} m\right) \phi_{ \pm}^{(2)}(k r)
\end{aligned}
$$


Substituindo as equaçöes (5.8.6) e (5.8.7) acima na equaçäo (5.8.3) temos:

$$
\left(\begin{array}{l}
\beta\left( \pm|E|+\sigma_{3} m\right) \phi_{ \pm}^{(2)} \\
\alpha\left( \pm|E|-\sigma_{3} m\right) \phi_{ \pm}^{(1)}
\end{array}\right)=\lambda\left(\begin{array}{ll}
\alpha & \phi_{ \pm}^{(1)} \\
\beta & \phi_{ \pm}^{(2)}
\end{array}\right)
$$

Assim, temos que:

$$
\begin{aligned}
& \beta\left( \pm|E|+\sigma_{3} m\right) \phi_{ \pm}^{(2)}=\lambda \alpha \phi_{ \pm}^{(1)} \\
& \alpha\left( \pm|E|-\sigma_{3} m\right) \phi_{ \pm}^{(1)}=\lambda \beta \phi_{ \pm}^{(2)} .
\end{aligned}
$$

A equaçăo (5.8.9) fornece o sistema de equaçoes abaixo:

$$
\begin{gathered}
\beta( \pm|E|+m)=\lambda \alpha, \\
\beta( \pm|E|+m) d_{ \pm}=\lambda \alpha c_{ \pm} .
\end{gathered}
$$

que evidentemente só tem solução sob a condição:

$$
c_{ \pm}=d_{ \pm}
$$

A equação (5.8.10) fornece o sistema de equaçöes:

$$
\begin{gathered}
\alpha( \pm|E|-m)=\lambda \beta \\
\alpha( \pm|E|-m) t_{ \pm}=\lambda \beta d_{ \pm}
\end{gathered}
$$

que evidentemente só tem soluçăo se satisfeita a mesma condiçäo da equaşão (5.8.13).

Sob a condição que $c_{ \pm}$seja igual a $d_{ \pm}$ternos, então, finalmente o sisterna:

$$
\begin{aligned}
& \beta( \pm|E|+m)=\lambda \alpha \\
& \alpha( \pm|E|-m)=\lambda \beta .
\end{aligned}
$$

A condicăo para existência de soluços para o sistema acima fornece:

$$
\lambda= \pm \sqrt{E^{2}-m^{2}}= \pm k,
$$

que são os auto-valores da helicidade. 
Para $\lambda=+h$, temos a seguinte relagäo entre os coeficiente $\alpha$ e $\beta$

$$
\rho=\frac{k}{ \pm|E|+m} \alpha
$$

e para $\lambda=-k_{\text {s }}$ temos:

$$
\beta=-\frac{k}{ \pm|E|+m} \alpha
$$

Assim tomando a e $\beta$ como nas equagoes $(3.8 .19)$ para $\lambda=+k=(5.8 .20)$ para $\lambda=-$ acima e com a condiçäo da equagäo (5.8.13) satisfeita num determinado domínio comum de $H$ $A$ obternas as auto-funcöes comulus desses operadores nesse domínio (veja Tabela 4).

\begin{tabular}{|l|l|l|}
\hline$\alpha=1$ & $\beta=\frac{k}{\mid E++m}$ & $\psi_{|E|, k}(k r)$ \\
\hline$\alpha=1$ & $\beta=-\frac{k}{|E|+m}$ & $\psi_{|E|,-k}(k r)$ \\
\hline$\alpha=1$ & $\beta=\frac{k}{-|Q|+m}$ & $\psi_{-|E|, k}(k r)$ \\
\hline$\alpha=1$ & $\beta=\frac{-k}{-|k|+m}$ & $\psi_{-|E|,-k}(k r)$ \\
\hline
\end{tabular}

Tabela 4 - Valores das constantes $\alpha$ e (que dajo as proporçöes das componentes superior e inferior do espinor) e as respextivass auto-funcŏes comuns aos operadores $H$ e $\Lambda$ obtidas.

\subsection{Auto-funçốes sỉmultâneas para os operadores $H$ e $\Lambda$ no domínio} $D_{\delta}(H, \Lambda)$ das condicöos de contorno desacopladas.

Os resultados obtidos na secäo anterior mostram que impor às auto-funços te $H$ encontradas na Segäo 5.5 (que obedecern ds condiçot de contorno desacopladas) que sejam também anto-fungós do operador $h$, equivale a tomar os coeficientes a $p$ conforme a Tabela 4 com a condiçäo da equaça (5.8.13) satisfeita, isto t:

$$
c_{ \pm}=d_{ \pm}
$$

Lembrando dos resultados obtidos na Tabela 2 da Seçể 5.5 , a equaça acima se torna:

$$
\frac{\operatorname{tg} \delta}{|E|+m}=\frac{\operatorname{tg} \delta}{|E|-m}
$$


a qual só tem solução se:

$$
8=0
$$

ou:

$$
\delta=\frac{\pi}{2}
$$

Para $\delta=0$, conforme podemos ver a partir da Tabela 2 , temos que a primeira componente do espinor de auto-fungäo fica sendo apenas a solução regular e a segunda componente fica sendo apenas a soluça singular na origem. Para $\delta=\frac{\pi}{2}$, conforme podemos ver a partix da Tabela 2, temos que a primeira componente do espinor de auto-fungäo fica sendo apenas a soluẹäo singular e a segunda componente fica sendo apenas a soluçäo regular na origem.

Assim, tomando $\delta=0$ (vemos que nesse caso $c_{ \pm}=d_{t}=0$ ), as auto-funcôes simultaneas de $H$ e $\Lambda$ säo:

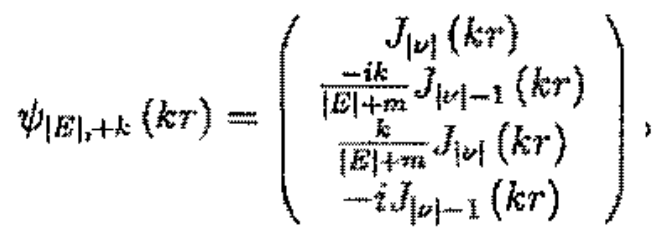

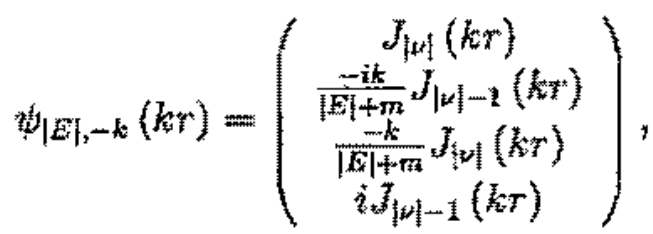

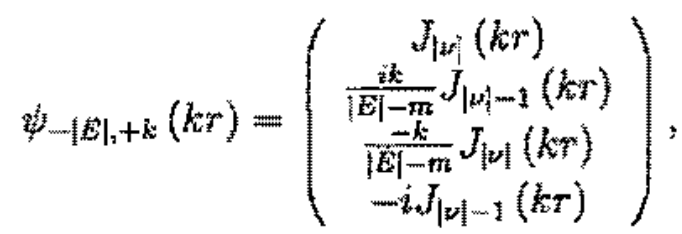

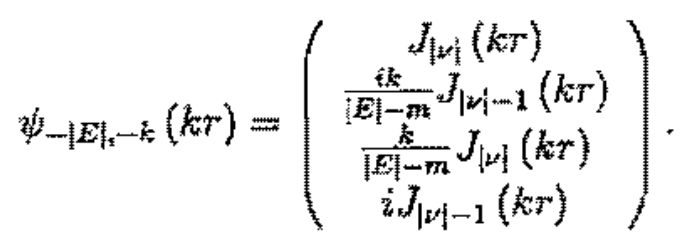


Tomando $\hat{b}=\frac{2}{2}$, as auto-funçôes simultâneas de $H$ e $\Lambda$ são:

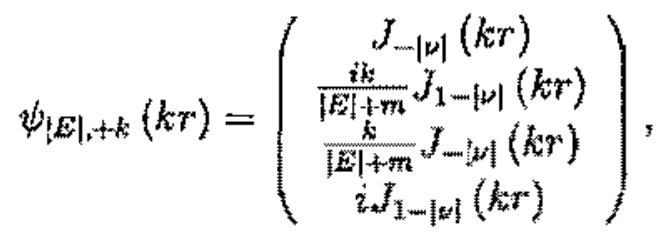

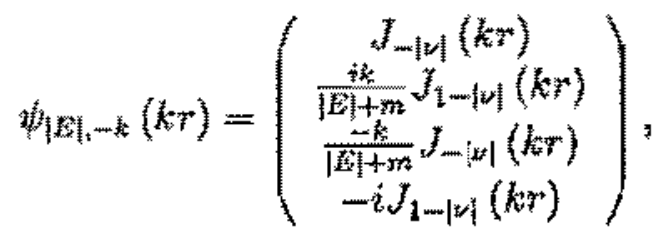

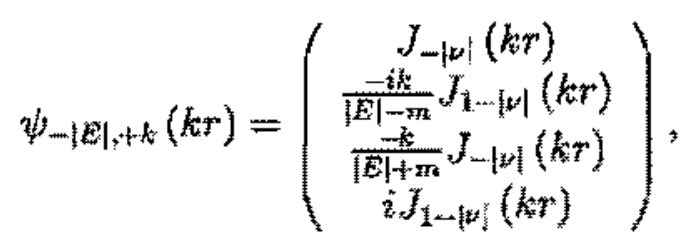

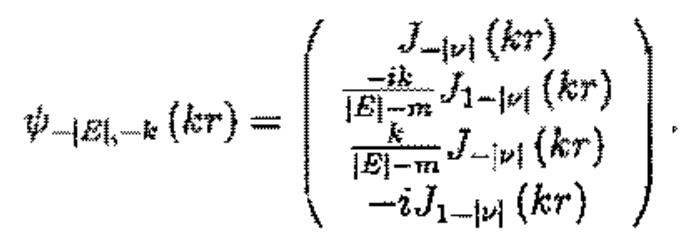

E interessante observar que as auto-funcoōes simultaneas obtidas nessa seyä incluem as apresentadas nas referencias [2] $e$ [4] obtidas através de um procedimento limite onde se substitui a linha de fuxo magnético na origem por um tubo de fluxo de rato $R>0$ (com o campo magnético $\bar{I}$ dado por uma funçăo do tipo 8 de casca) e toma-se o limitz $h \rightarrow 0$ no final dos cálculos.

Note que os resultados das equaşoes (5.9.1) a (5.9.4) implicam que a possibilidade de auto-funçôs comuns aos operadores $H$ e $A$ näo e garantida apenas pela definição dos dois operadores em um domínio comuxa onde ambos são autom adjuntos, como está sugerido na referência [1]. De modo que, só são possíveis auto-funcöes simultâneas a $H$ $\mathrm{\Lambda}$ apenas para certas extensöes particulares a um parâmetro: $\delta=0$ e $\delta=\frac{\pi}{2}$, que satisisfazem a condiçäo $(5.8 .13)$. 
5.10. A açäo do operador $\Lambda$ nas auto-funçöes de $H$ definidas em $D_{\delta}(H, \Lambda)$.

Um fato interessante que polemos perceber eque operador $\Lambda$ atuando em certas funçöes do dominio D. $(H, A)$ da Segão 5.4 gera funcous fora desse dominio, isto ê:

$$
A D_{\delta}=D^{\prime} \mid D^{\prime} \neq D_{\delta}
$$

Vejamos como isso acontece, deixando $A$ atuar numa anto-funça de $H$, escrita como ná equação $(5.5 .1)$, pertencente ao dominio $D_{S}(H, \Lambda)$, sujeita às condiçóes de contorno que produziram os resultados da Tabela 2. Assim:

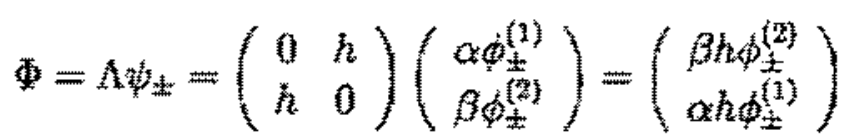

Irvocando os rasultados das equaceces $(5.8 .6)$ (5.8.7) e lembrando da forma das funçōes $\phi_{ \pm}^{(1)}$ e $\phi_{t}^{(2)}$ dadas pela equação (5.5.2) a (5.5.5), encontramas:

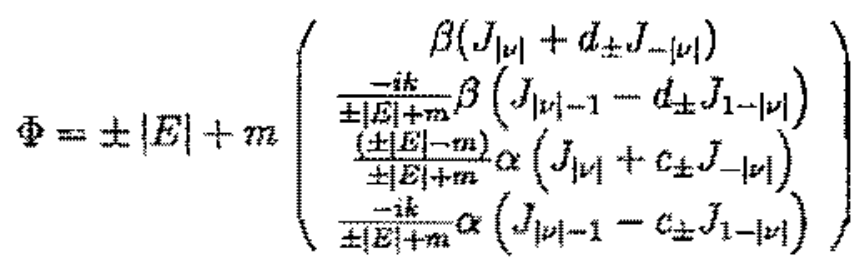

Veja que o par de componentes superior do espinor acima tem a mesma forma do espinor $\phi_{t}^{(1)}$ e o par de componentes inferior tem a mesme forma que a do espinores $\phi_{ \pm}^{(2)}$, no entanto as constantes $c_{ \pm}$e $d_{\text {s. }}$ aparecem em posígón trocadas. De forma que a funçäo $\Phi D_{\delta}(H, \Lambda)$, a menos que os coeficientes $c_{ \pm}$e $d \pm$ sejam ignais:

$$
c_{ \pm}=d_{ \pm}
$$

o que só ocorre am $D_{s}(H, \Lambda)$ na situagäo discutida na Secäo 5.9 , ou seja, quando o parâmetro $\delta$ da extensäo assume os valores $\delta=0$ e $\delta=\frac{\pi}{2}$. Aximin vemos que o operador $A$ ano mantem o dominio $D_{S}(H, \Lambda)$ invariante. Esse fato deve ter alguma analogia com o problema de Nelson ${ }^{[11]}$.

Podemos pensar, então, em estender o domínio comum dos operadores na tentativa de obter auto-funçóes comuns mais gerais para $H$ e $\Lambda$. 
Extender o dornínio, por outro lado, signiffen ter condigoes de contomo mais gerais que acoplem as quatro componentes dos espinores de atto-funços de $H$ e $\Lambda$, o que pode representar operacionalmente uma maior liberdade na escolha dos parâmetros da extensāo, viabilizando anto-funcốcs comuns a $H$ e $A$, que satisfazem a condição (5.8.13) e que săo diferentes das obtidas na Secăo 5.9 .

Além do operador $\Lambda$ näo deixar o dominio $D_{\delta}(H, \Lambda)$ invariante, também 0 operador $H$, atuando em certas funçôes do domínio $D_{S}(H, \Lambda)$, gera funções fora desse domínio, isto 6 :

$$
H D_{b}=D^{A t} \mid D^{t} \neq D_{\delta}
$$

Veja o Apêndice E. 


\section{Capítulo 6}

Extensōes auto-adjuntas mais gerais a dois parâmetros para o operador $H$ e conservação da helicidade.

\subsection{Determinação das condições de contorno.}

Nessa seçäo, vamos proceder a extensöes auto-adjuntas para o opersdor $H$ que impliquern em condiçöes de contorno que acoplem todas as componentes das funçōes de onda.

Vamos, entân, tomar a seguinte matriz paramétrica $W$ unitária:

$$
W=\left(\begin{array}{cc}
\cos \delta e^{i \omega} & -\operatorname{sen} \delta \\
\operatorname{sen} \delta & \cos \delta e^{-i \omega}
\end{array}\right)
$$

Assim as relações apresentadas na Tabela 1 ficam:

$$
\begin{aligned}
& \lim _{t \rightarrow 0} r\left[\varphi_{+}^{(1)}+\cos \delta e^{i \omega} \varphi_{m}^{(1)}-\operatorname{sen} \phi \varphi_{-}^{(2)}\right]^{\dagger} \alpha_{1} \psi=0 \\
& \lim _{t \rightarrow 0} r\left[\varphi_{+}^{(2)}+\operatorname{sen} \delta \varphi_{-}^{(1)}+\cos \delta e^{-i \omega_{\varphi}(2)}\right]_{m}^{\dagger} \alpha_{1} \psi=0
\end{aligned}
$$

Substituindo os resultados das equaçós $(5.1 .19)$ e $(5.1 .20)$, a equação $(6.1 .2)$ acima se toma:

$$
\lim _{r \rightarrow 0} r\left(\phi_{+}^{(1) \dagger}+\cos \delta e^{-i \phi^{(1)+\dagger}}\right) \sigma_{1}\left(\begin{array}{c}
\psi_{1} \\
\psi_{2}
\end{array}\right)-\lim _{r \rightarrow 0} r \operatorname{sen} \delta \phi_{-}^{(2) \dagger} \sigma_{1}\left(\begin{array}{l}
\psi_{3} \\
\psi_{4}
\end{array}\right)=0
$$


Com os expinores $\phi_{ \pm}^{(1)}$ e $\phi_{--}^{(2)}$ dados pelas equacöes (5.1.17) e (5.1.18) a equagöo scima se torna:

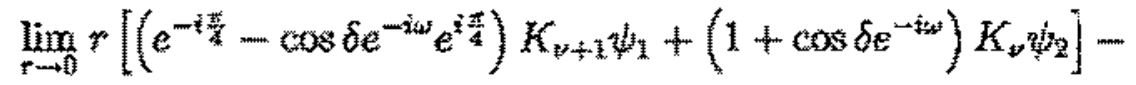

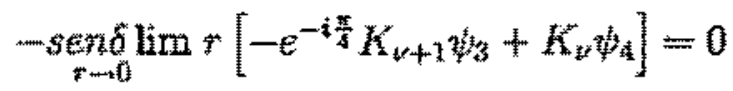

Lemabrando do comportamento na origem das funçós $K_{V}$ e $K_{t+1}$ dado pela equação (5.2.6) e usanto as definições de $x, y, z$ e $w$ dadas por $(5.4 .4)$ a (5.4.7) a equaça (6.1.5) acima pode ser escrita assim:

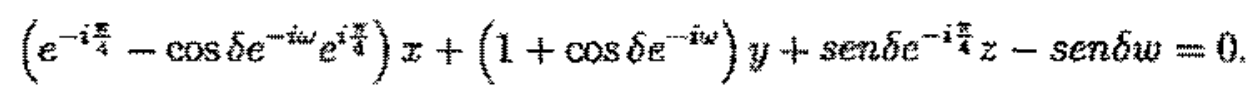

Procedendo em relaçăo à equação $(6.1 .3)$ da mesma forma que procedemos com a equaçäo (6.1.2) encontramos:

$$
-e^{i \frac{\pi}{i}} \operatorname{sen} \delta x+\operatorname{sen} \delta y+\left(e^{i \frac{\pi}{4}}-\cos \delta e^{i \omega} e^{-i \frac{\pi}{4}}\right) z+\left(1+\cos \delta e^{i \omega}\right) w=0
$$

As equacöes $(6.1 .6)$ e (6.1.7) substituen agora as condições de contorno das equações $(5.4 .2)$ e $(5,4.3)$ e acoplam todas as componentes das funçoes de onde.

Para maior comodidade, reescreveremos as equaçöes (6.1.6) e (6.1.7) da seguinte forma:

$$
\begin{gathered}
a x+b y+\operatorname{sen} \delta e^{-i \frac{z}{3} z-\operatorname{sen} \delta w}=0 \\
-e^{i \frac{\pi}{4}} \operatorname{sen} \delta x+\operatorname{sen} \delta y+a^{*} z+b^{*} w=0
\end{gathered}
$$

onde introduzimos as definições:

$$
\begin{gathered}
a=e^{-i \frac{\pi}{4}}-\cos b e^{-i \omega t} e^{i \frac{\pi}{4}} \\
b=1+\cos \delta e^{-i \omega s} .
\end{gathered}
$$

Note qute:

$$
b=(\sqrt{2}-a) e^{-i \frac{\pi}{4}}
$$


As exuaçöes (6.1.8) e (6.1.9) representam as condiçōes de contorno a serem satisfeitass pelas auto-funçoes de $H$ na origem, e definem novos domínios $D_{\delta, \omega}(H)$ para o operador $H$.

De outra forma, podemos dizer tambérn que as equaçōes (6.1.8) e (6.1.9) fornecern todas as extensōes auto-adjuntas a dois parâmetros para o hamiltoniana que acoplam todas as componentes das auto-funções.

\subsection{Determinaçăo das auto-funçōes de $H$ que satisfazen as condiçōes de contorno mais gerais a dois parâmetros.}

$\mathrm{Na}$ verdade, devemos nos lembrar que o nosso objetivo ao definirmos um domínio extendido para os operadores $H$ e $\Lambda$ foi o de verificar a possibilidade de obtermos auto-funçós simultâneas, com $H$ e $A$ sendo operadores auto-adjuntos nesse doxnínio.

No entanto, já vimos na Seçāo 5.8 que isso só é possível para certas combinaçōes lineares das auto-funçōes de $H$, apresentadas na Tabela 4, sob a condição da equação (5.8.13) ser satisfeita.

Seja, então, a auto-função de $H$ com energia pasitiva e possibilidade de $\lambda= \pm k$

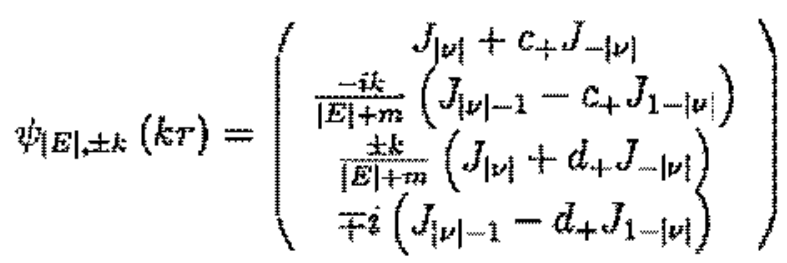

onde tomamos as constantes $\alpha$ e $\beta$ conforme a Tabela $4^{1}$.

Vamos verificar a possibilidade das auto-funçöes da forma da equaçăo anterior, satisfazendo as condiçöes de contorno das equaçöes (6.1.8) e (6.1.9), satisfazerem também a condiçâao da equaçäo (5.8.13), de forma a garantir que tais auto-fungônes de $H$ sejam tambéru auto-funçöes de $A$.

\footnotetext{
${ }^{1}$ Note que a fungäo

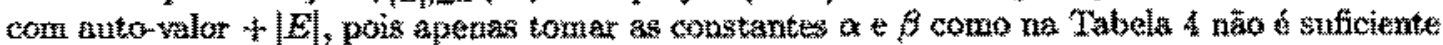

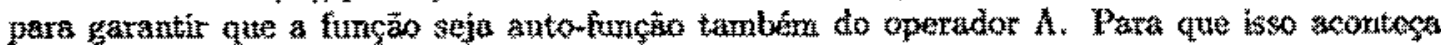

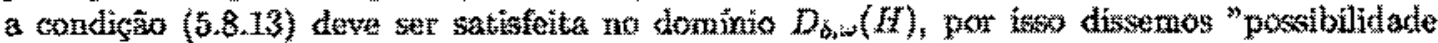

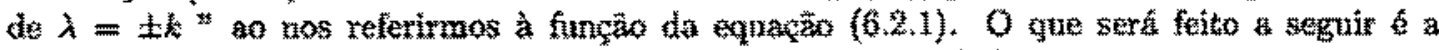

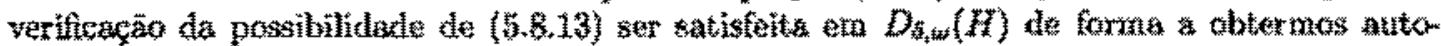

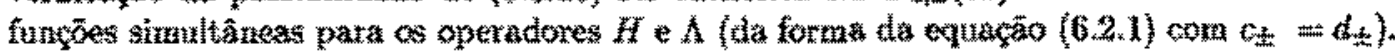


Lembrando do comportamento na origem das funçöes de Bessel, dado pela equaşa (5.2.6) e lembrando das definiçóes de $x, y, z$ e $w$ das equaçô (5.4.4) a (5.4.7), temos para a função da equaçăo (6.2.1) acima:

$$
\begin{aligned}
& x=(\sqrt{2} m)^{|m|-1} k^{-|m|} c_{+} \quad \\
& y=-i \frac{(\sqrt{2} m)^{-1 \omega i} k^{|\nu|}}{|E|+m} \\
& z= \pm(\sqrt{2 m})^{\mid \omega-1-1} k^{-|\nu|} \frac{k}{|E|+m} d_{+} \\
& w=\mp i(\sqrt{2} m)^{-i w \mid} \frac{k^{|w|}}{k}
\end{aligned}
$$

Substituindo as expressötes das equaçöes (6.2.2) a (6.2.5) nas equagōes (6.1.8) e (6.1.9) das condiçöes de contorno, obtemos:

$$
\begin{gathered}
\alpha c_{+}(\sqrt{2} m)^{|\nu|-1} k^{-|\nu|} \pm \operatorname{sen} \delta e^{-i \frac{\pi}{3}} \frac{k}{|E|+m} d_{*}(\sqrt{2} m)^{\mid \nu-1} k^{-|\nu|}= \\
=i(\sqrt{2} m)^{-|\mu|} k^{|v|}\left[\frac{b}{|E|+m} \mp \frac{\operatorname{sen} \delta}{k}\right]
\end{gathered}
$$

e:

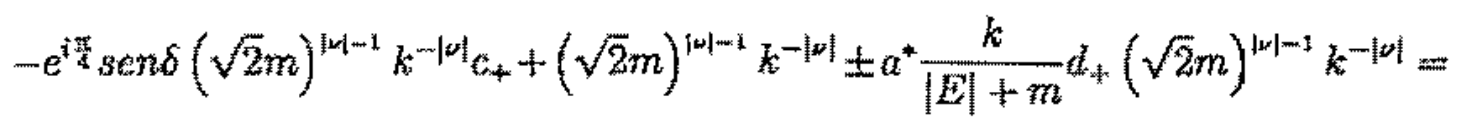

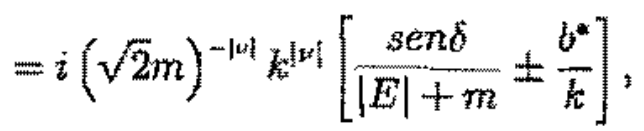

de onde devemos extrair $c_{+}$e $d_{+}$para depois verificar a possibilidade de satisfação da condiç̧̄o (5.8.13).

O sistema acima tera solucão apenas se:

$$
\cos \delta \operatorname{sen} \omega \neq 1
$$


Vamos aplicar a regra de Cramer para calcular os coeficientes $\epsilon_{+}$e $d_{+}$das equaçöes (6.2.6) e (6.2.7). Assim, sendo $\Delta, o$ determinante da matriz incomapleta do sistema de equaçöes $(6.2 .6)$ e $(6.2 .7)$, isto é:

$$
\Delta= \pm(\sqrt{2 m})^{2|m|-2} \frac{k^{-2|n|+2}}{|E|+m \mid}\left|-e^{i \frac{\pi}{4} \operatorname{sen} \delta} \quad a^{*+\frac{\pi}{4} \operatorname{sen} \delta}\right|
$$

temos:

$$
\Delta= \pm(\sqrt{2} m)^{2 \mid \omega t-3} \frac{k^{-2}|m|+1}{|a|+m}\left(a a^{*}+\operatorname{sen}^{2} \delta\right) .
$$

Vamos introduzir as definiçöes:

$$
\begin{aligned}
& f=a^{*} b-\operatorname{sen}^{2} \delta e^{-i \frac{u}{4}}, \\
& g=\left(a^{*}+b^{*} e^{-i \frac{*}{4}}\right) \operatorname{sen} \delta .
\end{aligned}
$$

Sendo $\Delta_{1}$ o deterorinante da matriz obtida de $\Delta$, substituindo-se a coluna dos coeficientes de $c_{+}$pela coluna dos termos independentes:

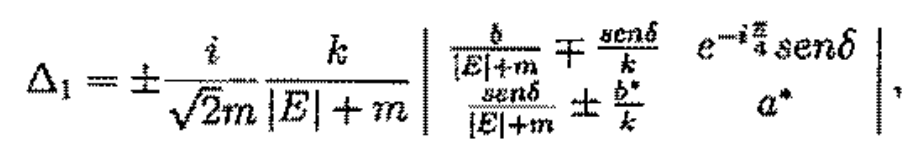

temos:

$$
\Delta_{1}= \pm \frac{i}{\sqrt{2} m} \frac{k}{|E|+m}\left\{\frac{f}{|E|+m} \mp \frac{g}{k}\right\}
$$

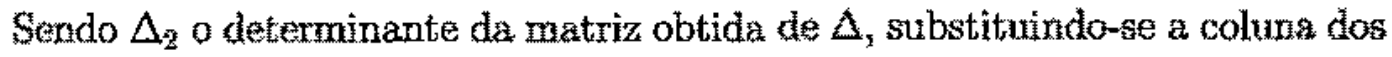
coeficientes de $d_{\%}$ pela coluna dos termos independentes:

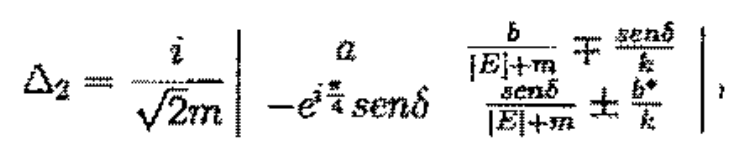

temos:

$$
\Delta_{z}=\frac{i}{\sqrt{2} m}\left\{\frac{g^{*}}{|E|+m} \pm \frac{f^{*}}{k}\right\}
$$


Substituindo as equaçöes (6.1.10) e (6.1.11) nas equaçös $(6.2 .10)$ e $(6.2 .11)$ acima, obtemos:

$$
f=i \sqrt{2}[1-\cos \delta(\operatorname{sen} \omega-\cos \omega)]
$$

e:

$$
y=\sqrt{2} \operatorname{sen} \delta \text {. }
$$

Podemos calcular $c_{+}$e $d+$ fazendo:

$$
c_{1}=\frac{\Delta_{1}}{\Delta}
$$

ex:

$$
d_{+}=\frac{\Delta_{2}}{\Delta}
$$

Como queremos que $c_{+}$seja igual a $d_{+}$(condiçäo da equacăo $(5.8 .13)$ ), vamos verificar a possibilidade de:

$$
\Delta_{1}=\Delta_{2}
$$

Com $\Delta_{1}$ e $\Delta_{2}$ dados pelas expressós $(6.2 .12)$ e $(6.2 .13)$, a expressän acima implica am:

$$
\pm \frac{h}{|E|+m}\left\{\frac{f}{|E|+m} \mp \frac{g}{k}\right\}=\frac{g^{*}}{|E|+m} \pm \frac{f^{*}}{k}
$$

a qual pode ser escrita como:

$$
\frac{ \pm k^{2} f-k(|E|+m)\left(g+g^{*}\right) \mp(|E|+m)^{2} f^{*}}{k(|E|+m)^{2}}=0
$$

Para que a equagão acima seja verificada para qualquer valor de $E_{;}$devemos ter:

$$
\begin{gathered}
f=f^{*}=0 \\
g=-g^{*}
\end{gathered}
$$


Substituindo a equaçäo (6.2.14) na expressâo (6.2.21) acima, obternos que:

$$
\cos \delta(\operatorname{sen} \omega-\cos \omega)=1
$$

Substituindo a equação (6.2.15) na expressäo $(6.2 .22)$ acima, obtemos:

$$
\operatorname{sen} \delta=-\operatorname{sen} \delta
$$

que áo é possível se:

$$
\delta=n \pi, n \in Z
$$

Assim, os parâmetros $\delta$ e $\omega$ da extensāo devern ser obtidos de modo a satisfazerern ìs condiçōes das equaçôes $(6.2 .8),(6.2 .23)$ e (6.2.25) simultaneamente.

Note que a condição da equação (6.2.25) implica que:

$$
y=-g^{*}=0 \text {. }
$$

De modo que o resultado da equaçäo acima junto com a equação (6.2.21) fornecem:

$$
c_{t}=d_{t}=0,
$$

que corresponde às mesmas soluçöes jâ anteriormente encontrada na Seçäo 5.9 do Capítulo 5 através das equaçōes (5.9.5) a (5.9.8)

Assim, as extensōes auto-sdjuntas mais gerais a dois parâmetros que acoplam todas as componentes das auto-funçōes näo produzem novas auto-funçōes simultâneas para os operadores $H \in \Lambda$, isto é, nẫo produzem auto-funcöes mais gerais que as já encontradas no Capítulo 5. Para obtermos auto-funģöes simultaneas mais gerais devemos proceder a extensōes auto-adjuntas mais gerais corn três e quatro parârnetros. Isso é o que faremos nos próximos capítulos. 


\section{Capítulo 7}

\section{Extensōes auto-adjuntas a três parâmetros para}

o operador Hamiltoniana e conservação da helicidade

7.1. Determinaçäo das extensöes auto-adjuntas a três parămetros para o operador Hamiltoniana.

Como näo conseguimos resultados novos com a extensão a dois parâmetros que mistura todas as componentes das funcōes de onda, varnos tentar extensōes a três parârnetros para o hamiltoniana.

Vamos entäo tomar a matriz W como sendo a matriz unitảia:

$$
W=\left(\begin{array}{cc}
\cos \theta e^{i \varphi} & i \operatorname{sen} \theta e^{-i \psi} \\
i \operatorname{sen} \theta e^{i \omega} & \cos \theta e^{-i t}
\end{array}\right) \text {. }
$$

De forma que as relaçöes apresentadas na Tabela 1 se tomam:

$$
\begin{aligned}
& \varliminf_{t \rightarrow 0} r\left[\varphi_{+}^{(1)}+\cos \theta e^{i \varphi^{\psi}} \varphi_{-}^{(1)}+\operatorname{sen} \theta e^{-i \phi} \varphi_{m}^{(2)}\right]^{\dagger} \alpha_{1} \psi=0
\end{aligned}
$$

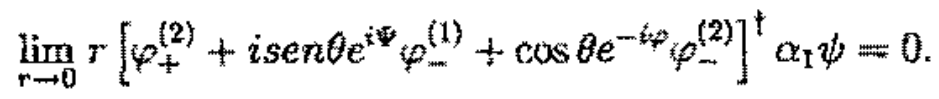

Substituindo os resultados das equaçöes (5.1.19) e (5.1.20), a equaçäo (7.1.2) acima se torna:

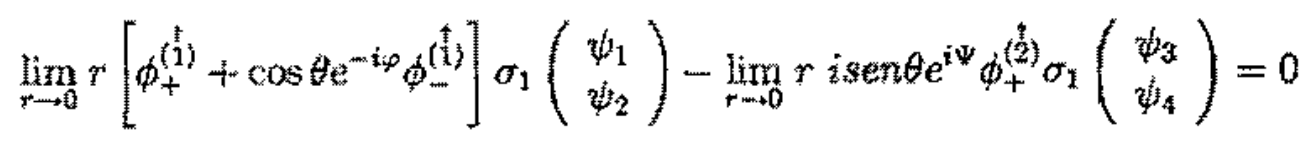


Usando os resultados das equaçes $(5.1 .17)$ e (5.1.18) a equaçõo acima se torna:

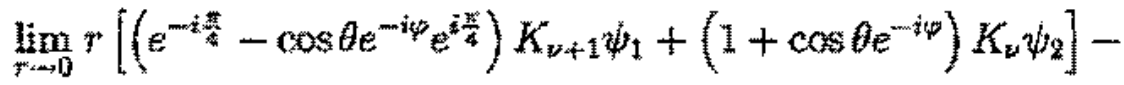

$$
\begin{aligned}
& -i \operatorname{sen} \theta e^{i \Psi} \lim _{t \rightarrow 0}\left(-e^{-i \frac{\pi}{4}} K_{*+1} \psi_{3}+K_{t} \psi_{4}\right)=0
\end{aligned}
$$

Lumbrando do comportamento na origem das funcōes $K_{y}$ e $K_{v+1}$ dado pela equaçäo (5.2.6) e usando as definiçöes de $x, y, z$ ew dadas por $(5.4 .4)$ a $(5.4 .7)$, a equaça acima fica:

$$
\begin{gathered}
\left(e^{\cdots i \frac{\pi}{4}}-\cos \theta e^{-i \varphi} e^{i \frac{k}{k}}\right) x+\left(1+\cos \theta e^{-i \varphi}\right) y+ \\
+\operatorname{sen} \theta e^{i \Psi} e^{-i \frac{\pi}{2}} z-i \operatorname{sen} \theta e^{i \omega} z=0 .
\end{gathered}
$$

Procedendo em relagăo à equaçäo $(7.1 .3)$ da mesma forma que levou a equaçäo (7.1.2) a poder ser escrita como na equasäo (7.1.6), encontramos:

$$
\begin{gathered}
i \operatorname{sen} \theta e^{-i \psi} e^{i \frac{\pi}{4} x}-i \operatorname{sen} \theta e^{-i \omega} y+\left(e^{i \frac{\pi}{4}}-\cos \theta e^{i \phi e^{-i \frac{\pi}{4}}}\right) z+ \\
+\left(1+\cos \theta e^{i \varphi}\right) w=0
\end{gathered}
$$

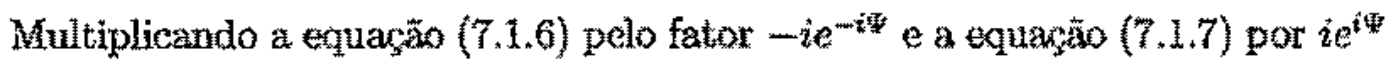
temos:

$$
\begin{gathered}
-i e^{-i \Psi}\left(e^{-i \frac{\pi}{4}}-\cos \theta e^{-i \varphi} e^{i \frac{\pi}{4}}\right) x-i e^{-i \omega}\left(1+\cos \theta e^{-i \varphi}\right) y \\
+\operatorname{sen} \theta e^{-i \frac{\pi}{2}} z-\operatorname{sen} \theta \omega=0 \\
-\operatorname{sen} \theta e^{i \frac{\pi}{4} x}+\operatorname{sen} \theta y+i e^{i \omega}\left(e^{i \frac{\pi}{4}}-\cos \theta e^{-i \frac{\pi}{4}}\right) z+ \\
+i e^{i \omega}\left(1+\cos \theta e^{i \varphi}\right) w=0
\end{gathered}
$$

Introduxindo as clefiniçoes:

$$
\begin{aligned}
& a=-i e^{-i \Psi}\left(e^{-i \frac{\pi}{4}}-\cos \theta e^{-i \phi} e^{i \frac{\pi}{4}}\right) \\
& b=-i e^{-i x}\left(1+\cos \theta e^{-i p}\right)
\end{aligned}
$$


as equacoces $(7.1 .8)$ e $(7.1 .9)$ se tornam:

$$
\begin{gathered}
a x+b y+\operatorname{sen} \theta e^{-i \frac{\pi}{4}} z-\operatorname{sen} \theta w=0 \\
-\operatorname{sen} \theta e^{i \frac{\pi}{4}} x+\operatorname{sen} \theta y+a^{*} z+b^{*} w z=0
\end{gathered}
$$

As equaços do sistema acima representam as condigôs de contomo a serem satisfeitas pelas auto-funçoes do hamiltoniana na origem e definem nowos domínios $D_{0, \Psi, \varphi}(H)$ para o operador $H$.

De outra forma, podemos dizer tambên que tais equaços $(7.1 .12)$ e (7.1.13) fornecem todas as extensôes auto-adjuntas a três parâmetros para o hamiltoniane.

7.2. Determinação das auto-funçöes de $H$ que satisfazem às condiçōes de contorno com três parâmetros.

Note que o sistema definido pelas exuacoes (7.1.12) e (7.1.13) tern a mesma forma do sisterna defindo pelas equagó $(6.1 .8)$ e $(6.1,9)$. Vamos tomat uma autom funçäo de $H$ com auto-valor de energia pasitivo com a mesma forma dada pela equacă (6.2.1), de modo que as quantidades $\pi, y, z$ e weräo dadas atraves das equacoes $(6.2 .2)$ a $(6.2 .5)$.

Substituindo ontāo as expressöes para $x, y, z$ e w nas equaçōes (7.1.12) e (7.1.13) das condiços de contorno obtemos o seguinte sistema de equacoes:

$$
\begin{aligned}
& a c_{+}(\sqrt{2} m)^{|\omega|+11} k^{-|m|} \pm \operatorname{sen} \theta e^{-i \frac{\pi}{3}} d+\frac{k}{|E|+m}(\sqrt{2} m)^{|m|-1} k^{-|\nu|}= \\
& =i(\sqrt{2} m)^{-\mid x i} k^{|n|}\left[\frac{b}{|E|+m} \pm \frac{\operatorname{sen} \theta}{k}\right]
\end{aligned}
$$

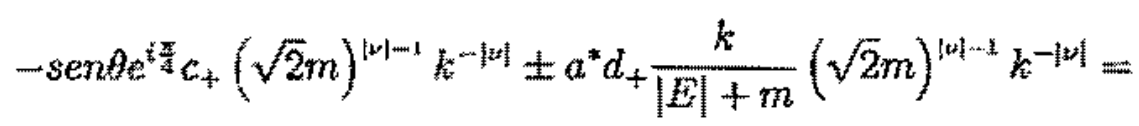

$$
\begin{aligned}
& =i(\sqrt{2} m)^{-|\omega|} k^{|v|}\left[\frac{\operatorname{sen} \theta}{|E|+m} \pm \frac{b^{*}}{k}\right]
\end{aligned}
$$

de onde deveruos extrair $c_{+}$ed para depois verificarmos a possibilidade de satisfaça da condicão $(5.8 .13)$. 
A condição para existência de soluçöes no sistema acima é :

$$
\cos \theta \operatorname{sente} \neq 1
$$

Varnos calcular os coeficientes $c_{+}$e $i_{+}$através da regra de Cramer. Assim, sendo $\Delta$ o determinante da matriz incompleta do sisterna de equações (7.21) e (7.2.2), temos:

$$
\Delta= \pm(\sqrt{2} m)^{2|x|-2} \frac{k^{m|q|+1}}{|E|+m}\left(a^{*} a+\operatorname{sen}^{2} \theta\right)
$$

Vamos introduzir as definições:

$$
\begin{gathered}
f=a^{*} b-\operatorname{sen}^{2} \theta e^{-i \frac{\pi}{4}} \\
g=\left(a^{*}+b^{*} e^{-i \frac{\pi}{4}}\right) \operatorname{sen} \theta .
\end{gathered}
$$

Sendo $\Delta_{1} \circ$ determinante da matriz obtida de $\Delta$ pela mudança da coluna dos conficientes de $c_{+}$pela coluna dos termos independentes, temos:

$$
\Delta_{1}= \pm \frac{i}{\sqrt{2} m} \frac{k}{|E|+m}\left\{\frac{f}{|E|+m} \mp \frac{g}{k}\right\} .
$$

Sendo $\Delta_{\text {c }} \mathrm{o}$ determinante da matriz obtida de $\Delta$ pela mudança da coluna dos coeficientes de $d_{+}$pela coluna dos termos independentes, temos:

$$
\Delta_{2}=\frac{i}{\sqrt{2} m}\left\{\frac{g^{*}}{|E|+m} \pm \frac{f^{*}}{k}\right\} .
$$

Com $a$ e $b$ dados, agora, pelas equaçóes (7.1.10) e (7.1.11) temos que as equaços $(7.2 .5)$ e $(7.2 .6)$ se tornam:

$$
\begin{gathered}
f=i \sqrt{2}(1-\cos \theta(\operatorname{sen} \varphi-\cos \varphi)) \\
g=i \sqrt{2} e^{i \omega} \operatorname{sen} \theta .
\end{gathered}
$$

Podemos calcular $c_{+}$e $d_{+}$fazendo:

$$
c_{+}=\frac{\Delta_{1}}{\Delta}
$$

e:

$$
d_{4}=\frac{\Delta_{2}}{\Delta}
$$


A fim de investigarmos agora a possibilidade de a condiçāo da equaxăo (5.8.13) ser satisfeita, isto é, de termos $c_{+}=d_{+}$vamos verificar a possibilidade de acontecer:

$$
\Delta_{1}=\Delta_{2}
$$

$\operatorname{Com} \Delta_{1}$ e $\Delta_{7}$ dados pelas equarötas (7.2.7) e (7.2.8), s expressão acima implica em termos:

$$
k^{2} f \mp k(E+m)\left(g^{*}+g\right)-(E+m)^{2} f^{*}=0
$$

Vemos que para a equaçào acima ser satisfeita para qualquer valor de $E$ devemos ter:

$$
\begin{gathered}
f=f^{*}=0 \\
g=-g^{*}
\end{gathered}
$$

Substituindo as equações (7.2.9) e (7.2.10) nas quaçōes acima obtemos as seguintes condiçôes:

$$
\cos \theta(\operatorname{sen} \varphi-\cos \varphi)=1
$$

e:

$$
e^{i \Psi}=e^{-i \Psi}
$$

A equaçăo (7.2.18) iroplica em:

$$
\Psi=n_{1} \pi \quad, n_{3}=0,1, \ldots
$$

Assim, a imposição da condiçẫo da equaçäo (5.8.13) para obtenção de autofunçôes comuns a $H$ e $\Lambda$ nos dominios $D_{\hat{q}, \psi}(H)$ caracterizados pelas equaçōes (7.1.12) e (7.1.13) conduz às relaçōes extre os parâmuetros das extensôes dadas pelas equaçōes $(7.2 .3),(7.2 .17)$ e (7.2.19), esquematizadas na tabela abaixo.

\begin{tabular}{|l|}
\hline $\cos \theta(\operatorname{sen} \varphi-\cos \varphi)=1$ \\
\hline $\cos \theta \operatorname{sen} \varphi \neq 1$ \\
\hline$\Psi=\pi n_{1} \quad n_{1}=0,1,2, \ldots$ \\
$\theta \neq \pi n^{\prime} \quad n^{\prime} \neq 0,1,2, \ldots$ \\
\hline
\end{tabular}

Tabela 5 - Relacöes que devem obedecer os trếs parâmetros das extensôes zuto-adjuntas para obtençäo de auto-funçöes comuns aos operadores $H$ e $\Lambda$. 
A última condigta da Tabela 5 fó colocada para que sejam obtidas solucoees diferentes das encontradas na segão 5.9 (isto eُ, para que tenhamos $c_{\text {. }}$, $d_{ \pm}$diferentes de zero).

Com as condiçōes da Tabela 5 , temos:

$$
\begin{gathered}
f=f^{*}=0 \\
g=-g^{*}=i \sqrt{2}(-1)^{n_{1}} \operatorname{sen} \theta .
\end{gathered}
$$

De modo que, as equaçōes $(7.2 .4),(7.2 .7)$ e (7.2.8), devido aos resultados das equacoōes acima ficam:

$$
\Delta_{1}=\Delta_{2}=\frac{1}{m(|E|+m)}(-1)^{n_{1}} \operatorname{sen} \theta
$$

e

$$
\Delta= \pm(\sqrt{2} m)^{2|\nu|-2} \frac{k^{-2|| \mid+1}}{|E|+m} 2(1-\cos \theta \operatorname{sen} \varphi) .
$$

Com os resultados das equaçón acima podemos obter $c_{+}$e $d_{+}$dados por (7.2.11) e (7.2.12) como sendo:

$$
c_{+}=d_{+}=+\frac{\sqrt{2}}{2} \frac{(-1)^{n_{1}} \operatorname{sen} \theta}{(1-\cos \theta \operatorname{sen} \varphi)}\left(\frac{k}{\sqrt{2} m}\right)^{2|\omega|-1},
$$

$\sec \lambda=+k$ e:

$$
c_{+}=a_{+}=-\frac{\sqrt{2}}{2} \frac{(-1)^{n_{1}} \operatorname{sen} \theta}{(1-\cos \theta \operatorname{sen} \varphi)}\left(\frac{k}{\sqrt{2} m}\right)^{2|\omega|-1}
$$

$\sec \lambda=-k$.

Observando a forma das auto-funcôes de $H$ dada pelas equaçóes (5.5.1) a (5.5.5) e a forma dos coeficientes $\alpha$ e $\beta$ (Tabela 4), vemos que para obter as autofunçōes simultâneas de $H$ e $\Lambda$ com energia negativa, basta trocar $|E| \rightarrow-|E|$. Da mesma forma para se obter as constantes $c_{-}$e $d_{-}$basta trocar $|E| \rightarrow-|E|$ na equaşöes $(7.2 .24 \mathrm{a})$ e $(7.2 .24 \mathrm{~b})$. 
Desse modo, considerando os resultado obtidos nesse secão, teremos as seguintes auto-funçöes simultatueas para $H$ e $A$, apresentadas aa Tabela 6 abaixo, com os parâmetros da extensäo relacionados conforme mostra a Tabela 5:

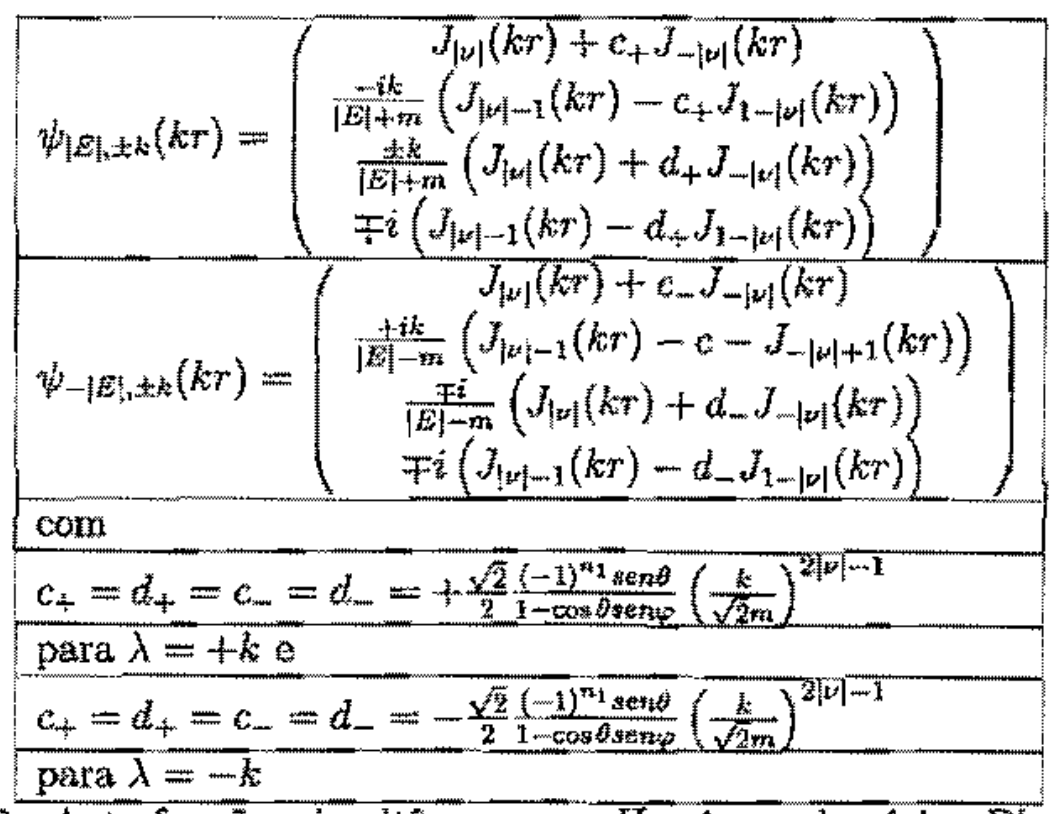

Tabela 6 - Auto-funçōes simultắneas para $H$ e $\mathrm{A}$ nos domínios $D_{0,4, \omega}^{F}(H, A)$ caracterizados pelas equaçöes (7.1.12) e (7.1.13), com os parâmetros das extensões satisfazendo as condiçoes da Tabela 5 .

As auto-funçôes da Tabela 6 sẫo antofunçồes simultâneas dos operadores $H$ e $A$, ambos definidos nos domínios $D_{\theta, 4}^{\prime}(H)$, definidos pelas equaçöes $(7.1 .12)$ e (7.1.13) e com as condigōes da Tabela 5, onde $H$ é anto-adjunto.

Surge entāo a questäo crucial:

"Será qué A é auto-adrunto nesses domíños?". 
7.3. Verificando se $A$ e auto-adjunto no domínio $D_{0, \psi, q}(H, \Lambda)$ encontrado. Varnos verificar se o operador $A$ e auto-adjunto no domínio $D_{t, \Phi_{i}}^{t}(H, A)$ definido pelas quaço (7.1.12) e (7.1.13) com as condiçores da Tabela

Ja vimos no Capítulo 5 , que as condiçoes de contorno mais gerais a serem satisfeitas pelas auto-funçöes de $\Lambda$ na origem que correspondem a todas as possiveis extensôes automadjuntas de $\Lambda$, parametrizadas pela matriz $V$ unitária, são dadas pelas equacôtes $(5.3 .11)$ e $(5.3 .12)$ abaixo transcritas:

$\left(1+\bar{v}_{11}+i \bar{v}_{12}\right) w+\left(1-\bar{v}_{11}+i \mathrm{v}_{12}\right) z+\left(1+\bar{v}_{11}-i \bar{v}_{12}\right) y+\left(1-\bar{v}_{11}-i \bar{v}_{12}\right) x=0$

$\left(1+\overline{\mathrm{v}}_{21}+\mathrm{v}_{22}\right) w+\left(-i-\overline{\mathrm{v}}_{21}+i \overline{\mathrm{v}}_{22}\right) z+\left(-i+\overline{\mathrm{v}}_{21}-i \overline{\mathrm{v}}_{22}\right) y+\left(i-\overline{\mathrm{v}}_{21}-i \overline{\mathrm{v}}_{22}\right) x=0$

Queremos defuir $\Lambda$ no domínio $D_{\theta, \Psi, 4}^{\prime}(H, \Lambda)$, caracterizados pelas equaçöes (7.1.12) e (7.1.13) abaixo transcritas:

$$
\begin{gathered}
a x+b y+\operatorname{sen} \theta e^{-i \frac{\pi}{4}} z-\operatorname{sen} \theta w=0 \\
-e^{i \frac{\pi}{4} \theta} \operatorname{sen} \theta x+\operatorname{sen} \theta y+a^{*} z+b^{*} w=0,
\end{gathered}
$$

onde, devido as condigós da Tabela 5 podemos escrever as equaçoes (7.1.10) e (7.1.11) como sendo:

$$
\begin{gathered}
a=-(-)^{n_{1}}\left(e^{-i \frac{\pi}{4}}-\cos \theta e^{-i \phi_{p}} e^{i \frac{\pi}{4}}\right) \\
b=-i(-)^{n_{1}}\left(1+\cos \theta e^{-i \varphi}\right)
\end{gathered}
$$


O sistema de equaçöes (7.3.3) e (7.3.4) das condiçố de contorno sob as condiģoes da Tabela 5 , pode ser escrito como:

$$
\begin{gathered}
x=m_{\theta} \operatorname{sen} \varphi w \\
y=m_{\theta} \cos \varphi z
\end{gathered}
$$

onde:

$$
m_{\theta}=i \sqrt{2}(-)^{n_{1}} \cot \theta .
$$

Lernbremo-nos que os parâmetros $\theta$ e $\varphi$ estäo relacionados conforme mostra a Tabela 5 .

Substituindo as equaciones $(7.3 .7)$ e (7.3.8) na equaçäo (7.3.1) obtemos :

$$
\begin{array}{r}
{\left[1+m_{\theta} \operatorname{sen} \varphi+\bar{v}_{11}\left(1-m_{\theta} \operatorname{sen} \varphi\right)+\hat{v}_{12}\left(1-m_{\theta} \operatorname{sen} \varphi\right)\right] w+} \\
+\left[1+m_{\theta} \cos \varphi-\bar{v}_{11}\left(1-m_{\theta} \cos \varphi\right)+i v_{12}\left(1-m_{\theta} \cos \varphi\right)\right] z=0
\end{array}
$$

Para que a equaşo acima serifique para qualquer $w$ e $z$, devemos ter:

$$
\begin{aligned}
& \overrightarrow{\mathrm{v}}_{11}\left(1-m_{\theta} \operatorname{sen} \varphi\right)+i \overline{\mathrm{v}}_{12}\left(1-m_{\theta} \operatorname{sen} \varphi\right)=-\left(1+m_{\theta} \operatorname{sen} \varphi\right) \\
& \overline{\mathrm{v}}_{11}\left(1-m_{\theta} \cos \varphi\right)-i \overline{\mathrm{v}}_{12}\left(1-m_{\theta} \cos \varphi\right)=\left(1+m_{\theta} \cos \varphi\right)
\end{aligned}
$$

$O$ sistema acina sempre terá soluçẫo:

$$
\begin{aligned}
& \nabla_{11}=\frac{-m_{\theta}(\operatorname{sen} \varphi-\cos \varphi)}{\left(1-m_{\theta} \operatorname{sen} \varphi\right)\left(1-m_{\theta} \cos \varphi\right)} \\
& \overline{\mathrm{v}}_{12}=\frac{i\left(1-m_{\theta}^{2} \operatorname{sen} \varphi \cos \varphi\right)}{\left(1-m_{\theta} \operatorname{sen} \varphi\right)\left(1-m_{\theta} \cos \varphi\right)}
\end{aligned}
$$

Da mesma forna, substituindo as equaçöes (7.3.7) e (7.3.8) na equaçào (7.3.2) obtemos:

$$
\begin{array}{r}
{\left[i\left(1+m_{\theta} \operatorname{sen} \varphi\right)+\overline{\mathrm{v}}_{21}\left(1-m_{\theta} \operatorname{sen} \varphi\right)+i \bar{y}_{22}\left(1-m_{g} \operatorname{sen} \varphi\right)\right] w+} \\
{\left[-i\left(1+m_{\theta} \cos \varphi\right)-\overline{\mathrm{v}}_{21}\left(1-m_{\theta} \cos \varphi\right)+i \overline{\mathrm{v}}_{22}\left(1-m_{\theta} \cos \varphi\right)\right] z=0 .}
\end{array}
$$


Para que a equaça acima seja sempre verificada para qualquer $w$ e $z$, devemos ter que:

$$
\begin{array}{r}
\overline{\mathrm{v}}_{21}\left(1-m_{\theta} \operatorname{sen} \varphi\right)+\overline{\mathrm{v}}_{22}\left(1-m_{\theta \operatorname{sen}} \varphi\right)=-i\left(1+m_{\theta} \operatorname{sen} \varphi\right) \\
\overline{\mathrm{Y}}_{21}\left(1-m_{\theta} \cos \varphi\right)-\mathrm{i}_{22}\left(1-m_{\theta} \cos \varphi\right)=-i\left(1+m_{\theta} \cos \varphi\right)
\end{array}
$$

Esse sistema sempre terá soluçăo:

$$
\begin{aligned}
\overline{\mathrm{v}}_{21} & =\frac{-\left(1-m_{\theta}^{2} \operatorname{sen} \varphi \cos \varphi\right)}{\left(1-m_{\theta} \operatorname{sen} \varphi\right)\left(1-m_{\theta} \cos \varphi\right)} \\
\mathrm{v}_{22} & =\frac{-m_{\theta}(\operatorname{sen} \varphi-\cos \varphi)}{\left(1-m_{\theta} \operatorname{sen} \varphi\right)\left(1-m_{\theta} \cos \varphi\right)} .
\end{aligned}
$$

As equaço $(7.3 .13)$ (7.3.19) implicam que:

$$
\mathrm{v}_{11}=\overline{\mathrm{v}}_{2 \mathrm{i}}
$$

Da mesma forma a partir das equagón (7.3.14) e (7.3.18) vemos que:

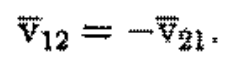

Notemos também, através da definição $(7.3 .9)$, que

$$
m_{i}^{*}=-m_{\theta}
$$

Assim, podemos escrever os elementos da matriz $V$, como sendo;

$$
\begin{aligned}
v_{11} & =\mathrm{v}_{22}=\frac{m_{0}(\operatorname{sen} \varphi-\cos \varphi)}{\left(1+m_{\theta} \operatorname{sen} \varphi\right)\left(1+m_{\theta} \cos \varphi\right)} \\
v_{12} & =-\mathrm{v}_{21}=\frac{-i\left(1-m_{\theta}^{2} \operatorname{sen} \varphi \cos \varphi\right)}{\left(1+m_{\theta} \operatorname{sen} \varphi\right)\left(1+m_{\theta} \cos \varphi\right)}
\end{aligned}
$$


Vejarnos, agora, sob que condisōes a matriz $V$ é unitária, isto é:

$$
\begin{gathered}
V V^{-1}=1 \\
\left(\begin{array}{cc}
v_{11} & v_{12} \\
-v_{12} & v_{11}
\end{array}\right)\left(\begin{array}{cc}
\bar{v}_{12} & -\bar{v}_{12} \\
\bar{v}_{12} & \bar{v}_{11}
\end{array}\right)=\left(\begin{array}{ll}
1 & 0 \\
0 & 1
\end{array}\right) \\
{\left[\begin{array}{cc}
v_{11} \bar{v}_{11}+v_{12} v_{12} & -v_{11} \bar{v}_{12}+\bar{v}_{11} v_{12} \\
-\bar{v}_{11} v_{12}+v_{11} \bar{v}_{12} & v_{12} \bar{v}_{12}+v_{11} \bar{v}_{11}
\end{array}\right]=\left(\begin{array}{cc}
1 & 0 \\
0 & 1
\end{array}\right)}
\end{gathered}
$$

Assim, devemos ter que:

$$
\begin{aligned}
& v_{11} \bar{v}_{12}+v_{12} \bar{v}_{12}=1 \\
& v_{11} \bar{v}_{12}-\bar{v}_{11} v_{12}=0 .
\end{aligned}
$$

A partir das equaçồ (7.3.23) e (7.3.24) podemos calcular:

$$
\begin{aligned}
& v_{11} \bar{v}_{11}=\frac{-m_{\theta}^{2}(\operatorname{sen} \varphi-\cos \varphi)^{2}}{\left(1-m_{\theta}^{2} \operatorname{sen}^{2} \varphi\right)\left(1-m_{\theta}^{2} \cos ^{2} \varphi\right)} \\
& v_{12} \bar{v}_{12}=\frac{\left(1-m_{\theta}^{2} \operatorname{sen} \varphi \cos \varphi\right)^{2}}{\left(1-m_{\theta}^{2} \operatorname{sen}_{\theta}^{2} \varphi\right)\left(1-m_{\theta}^{2} \cos ^{2} \varphi\right)}
\end{aligned}
$$

De modo que:

$$
v_{11} \bar{v}_{11}+v_{12} \bar{v}_{12}=1
$$

attomaticamente.

Da mesma forma, utulizando as equaçoes $(7.3 .23)$ e (7.3.24) podemos calcular:

$$
\begin{aligned}
& \mathrm{v}_{11} \mathrm{v}_{12}=\frac{i m(\operatorname{sen} \varphi-\cos \varphi)\left(1-m_{\theta}^{2} \operatorname{sen} \varphi \cos \varphi\right)}{\left(1-m^{2} \operatorname{sen}^{2} \varphi\right)\left(1-m_{\theta}^{2} \cos ^{2} \varphi\right)} \\
& \nabla_{11} v_{12}=\frac{i m(\operatorname{sen} \varphi-\cos \varphi)\left(1-m_{\theta}^{2} \operatorname{sen} \varphi \cos \varphi\right)}{\left(1-m_{2}^{2} \operatorname{sen}^{2} \varphi\right)\left(1-m_{\theta}^{2} \cos ^{2} \varphi\right)}
\end{aligned}
$$


De modo que:

$$
\overline{\mathrm{v}}_{11} \mathrm{v}_{12}-\mathrm{v}_{11} \overline{\mathrm{v}}_{12}=0
$$

automaticamente.

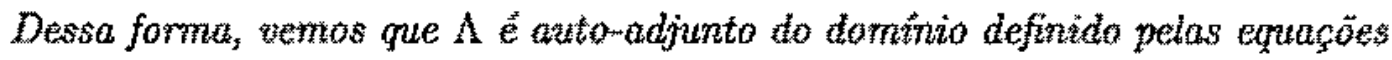
(7.1.19) e (7.1.19) sob as condiçoes da Tabela 5 que relacionam os parämatros da extensato, nato havendo qualquer outra restricto adicional a ser satisfeita mama que $\Lambda$ seja automadjunto nesse domínio.

Portanto $\Lambda$ e $H$ combtam e consegtentemente admitem auto funcões simultaneas

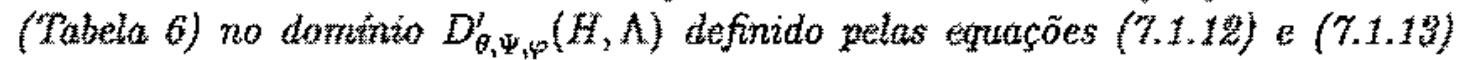
com as condiçes da Tabela 5, onde ambos sâo atto-adjuntos. Em outrus palamos, as dinâmicas defnidas atrovés das equaçōes (7.1.12) e (7.1.13) para H com as condiçôes da Tabela 5 conservam a helicidade.

\subsection{Exemplo: Uma extensäo particular a três parâmetros.}

Uma maneira particular de satisfazermos as condiçôs da Tabela 3 é tomarmos os parâmetros da extensệo como sendo:

$$
\begin{gathered}
\theta=\frac{\pi}{4} \\
y=\frac{3 \pi}{4} \\
\Psi=0 .
\end{gathered}
$$

Com esses valores para os parametros as equacou (7.1.12) e (7.1.13) das condiçöos de contorno podem ser esteritas como:

$$
\begin{gathered}
-x-i e^{-i \frac{\pi}{4}} y+z^{-i \frac{x}{3}}-w=0 \\
-e^{+i \frac{x}{4} x+i e^{-i \frac{x}{4}} y-e^{-i \frac{x}{4}} z-w}=0
\end{gathered}
$$


Usando as definiģöes das equações $(7.2 .6)$ e (7.2.7), obtemos que:

$$
\begin{gathered}
f=f^{*}=0 \\
g=-g^{*}=-\hat{2}
\end{gathered}
$$

De modo que, as equaçoses $(7.2 .7),(7.2 .8)$ e (7.2.4) fornecem para uma autofunção de $H$ com energia positiva e $\lambda= \pm k$, dada através da equaşăo (6.2.1):

$$
\Delta_{1}=\Delta_{2}=\frac{1}{\sqrt{2} m} \frac{1}{\{|E|+m)}
$$

e:

$$
\Delta= \pm(\sqrt{2} m)^{2|m|-2}\left(\frac{k}{|E|+m}\right)^{-2|m|+2}
$$

E, portanto, temos:

$$
\begin{gathered}
c_{+}=d_{+}=\frac{\Delta_{1}}{\Delta}=\frac{\Delta_{2}}{\Delta} \\
c_{+}=d_{+}=+\left(\frac{k}{\sqrt{2 m}}\right)^{2, j p l-z}
\end{gathered}
$$

$\operatorname{para} \lambda=+k$ e:

$$
c_{+}=d_{+}=-\left(\frac{k}{\sqrt{2} m}\right)^{2|v|-1}
$$

$\operatorname{para} \lambda=-\hbar$.

Assim, as auto-funçoes de $H$ da forma da equação (6.2.1), com $c_{+}$e $d_{+}$dados pelas equaçöes (7.4.10a) e (7.4.10b) acima satisfazem às condicōes de contorno dadas pelas equaçó (7.4.4) e (7.4.5) (para os valores dos parâmetros dados pelas equaçöes (7.4.1) e (7.4.3)) e correspondem a auto-funçöes simultâness de $H$ e $\Lambda$, uma vez que satisfazem a condisăo da equação (5.8.13).

Observando a forma das auto-funçöes de $H$ dada pela equasào (5.5.1) e a forma dos coeficientes $\alpha$ e $\beta$ dados na Tabela 4 , vemos que para obter autofunçōes sitnultäneas de $H$ e $A$ com energia negativa basta trocar $|E| \rightarrow-|E|$ 
nas auto-funçös simultảneas de energia positiva (equação (6.2.1)). Da mesma forma para se obter as constantes $c_{-} e d_{-}$basta trocar $|E| \rightarrow-|E|$ na equaçóes $(7.4 .10 a)$ e $(7.4 .10 b)$.

Assim, teremos as seguintes auto-funçós simultâneas de $H$ \& $\Lambda_{\text {, dadas }}$ na Tabela 7 que pertencem ao domínio $D_{\frac{2}{6}, 0, \frac{\pi}{4}}^{t}(H, \Lambda)$, onde cstamas definindo os operadores $H \in A$.

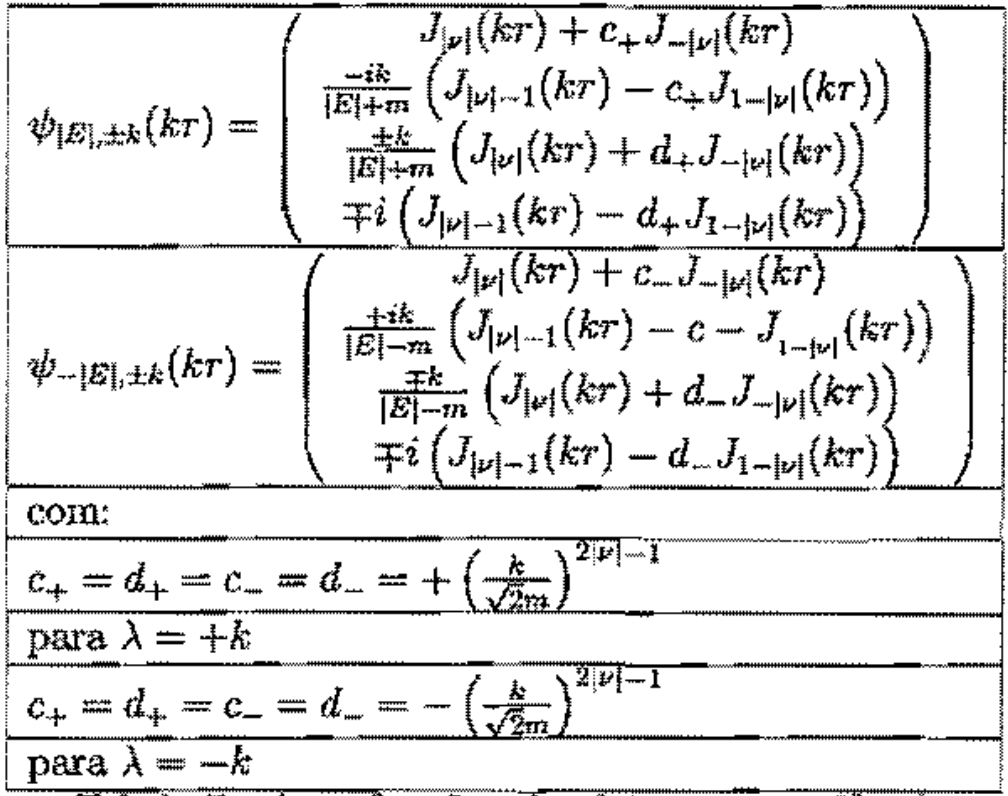

$$
\begin{aligned}
& \text { Tabela } 7 \text { - Anto-funco simulasueas para } H \text { e } A \\
& \text { no domínio } D_{\frac{\pi}{3}, 0, \frac{a g}{4}}(H, \Lambda) \text {. }
\end{aligned}
$$

Agora, resta cherar se $A$ é auto-adjunto no domínio em questâo.

7.5. Verificando se o operador $A$ é auto-adjunto no dominio $D_{\frac{\pi}{4}, 0, \frac{7 \pi}{4}}^{\prime}(H, A)$.

Yâ vimos no Capitulo 5 , qute as condiçôs de contorno màs gerais a serem satism feitas pelas auto-funcós de $\Lambda$ na origem, parametrizadas pela matriz $V$ unitária, säo dadas pelas equaçós $(5.3 .11)$ e (5.3.12) e correspondem a todas as possiveis extensōes auto-adjuntas para a helicidade.

Para sabtarmos se $A$ a auto-adjunto no dominio definido pelas equaçōes (7,4.4) e (7.4.5), basta impormos que o sistema de equacöes (5.3.11) e (5.3.12) seja equivm alente, isto é, tenha o mesmo coujunto de soluçóes que o sistema das equaçōes $(7.4 .4)$ e $(7.4 .5)$, e verificarmos se isso resulta em uma matriz paramétrica $V$ 
unitária, Se isso acontecer, entăo $A$ é auto-adjunto, caso contrário, $A$ näo é autoadjunto no dominio em questäo. Vejamos.

As extensöes anto-adjuntac mais gerais para a helicidade são dadas pelas equacöos $(5.3 .11)$ e (5.3.12).

Queremos verificar se $\Lambda$ é auto-adjunto no dominio $D_{\frac{3}{4}, 0, \frac{3 \pi}{6}}^{i}(H, \Lambda)$ caracterizado através das equações $(7.4 .4)$ e $(7.4 .5)$.

Das equatoses (7.4.4) e (7.4.5) podemos escrever:

$$
\begin{aligned}
& x=i w \\
& y=-i z
\end{aligned}
$$

Substitundo as equaços $(7.5 .1)$ e (7.5.2) nas equaçós $(5.3 .11)$ e (5.3.12) e após manipulaçós matemáticas, obtemos:

$$
\begin{gathered}
\left(e^{i \frac{\pi}{4}}+\bar{v}_{11} e^{-i \frac{\pi}{4}}+v_{12} e^{i \frac{\pi}{4}}\right) w+\left(e^{-i \frac{\pi}{4}}-\bar{v}_{12} e^{i \frac{\pi}{4}}-\bar{v}_{12} e^{-i \frac{x}{4}}\right) z=0 \\
\left(-e^{-i \frac{\pi}{4}}+\bar{v}_{21} e^{-i \frac{\pi}{4}}+\bar{v}_{22} e^{i \frac{\pi}{4}}\right) w+\left(-e^{i \frac{\pi}{4}}-\bar{v}_{21} e^{i \frac{\pi}{4}}-\bar{v}_{22} e^{-i \frac{\pi}{4}}\right) z=0
\end{gathered}
$$

Para que as equacoces acima sejam verificadas para qualquer $w$ e $z$, devemos tert que:

$$
\begin{aligned}
& \overline{\mathrm{v}}_{11} e^{-i \frac{\pi}{4}}+\overline{\mathrm{v}}_{12} e^{i \frac{\pi}{4}}=-e^{i \frac{\pi}{4}} \\
& \overline{\mathrm{v}}_{11} e^{i \frac{\pi}{4}}+\overline{\mathrm{v}}_{12} e^{-i \frac{\pi}{4}}=e^{-i \frac{\pi}{4}} \\
& \overline{\mathrm{v}}_{21} e^{-i \frac{\pi}{4}}+\overline{\mathrm{v}}_{22} e^{i \frac{\pi}{4}}=e^{-i \frac{\pi}{4}} \\
& \overline{\mathrm{v}}_{21} e^{i \frac{\pi}{4}}+\overline{\mathrm{v}}_{22} e^{-i \frac{\pi}{4}}=-\mathrm{e}^{i \frac{\pi}{4}}
\end{aligned}
$$


As equacōes acima correspondem a dois sistema de equaçöes desacopladas, de onde thramos que:

$$
v_{11}=v_{22}=
$$

e

$$
v_{12}=v_{21}=0
$$

dando a matriz $V$ :

$$
V=\left(\begin{array}{ll}
i & 0 \\
0 & i
\end{array}\right)
$$

que é unitária $\left(V V^{-1}=1\right)$.

Portanto, podemos concluir que $A$ é adjumto no dominio $D_{\frac{x}{4}, 0,3 x}^{t}\left(\frac{x}{4}, A\right)$ dado pelas equações $(7.4 .4)$ e $(7,4.5)$.

Assim, vemos que, de fato, as condiçôes de contorno dadas pelas equagōes (7.4.4) e (7.4.5) fornecem um possível domímio onde os operadores $H$ e $A$ säo auto-adjuntos e efetivamente comutam. De modo que săo possíveis auto-funçôes comuns (veja Tabela 7). Em outras palavras, a dinâmica definida pelas equaçōes $(7.4 .4)$ e $(7.4 .5)$, de tato, conserva a helicidade. 


\section{Capítulo 8}

Extensōes auto-adjuntas mais geraís a quatro parämetros para o operador $H$ e conservação da helicidade

8,1. Determinação das condiçōes de contorno mais gerais com quatro parâmetros para o operador Hamiltoniana.

Nessa seçāo, vamos proceder a extensão anto adjunta mais geral para o operador $H$ que ímplica em condiçôes de contomo parametrizadas por quatro parämetros e que acoplarn todas as quatro componentes dos espinores das hunçöes. Vamos, entäo, tomar a seguinte matsiz paramétrica $W$ unitárias:

$$
W=\left(\begin{array}{cc}
\cos \theta e^{i(\varphi+\alpha)} & i \operatorname{sen} \theta e^{-i(\Psi-\alpha)} \\
i \operatorname{sen} \theta e^{i(\Psi+\alpha)} & \cos \theta e^{-i(\varphi-\alpha)}
\end{array}\right)
$$

De modo cue as duas primeiras equações apresentadas na Tabela 1 ficam:

$$
\begin{aligned}
& \lim _{t \rightarrow 0} r\left[\psi_{+}^{(n)}+\cos \theta e^{i(\rho+\alpha)} \varphi_{m}^{(1)}+i \operatorname{sen} \theta e^{-i(\psi+\alpha)} \varphi_{-}^{(2)}\right]^{\dagger} \alpha_{1} \psi=0 \\
& \lim _{\tau \rightarrow 0} r\left[\varphi_{\psi}^{(2)}+i \operatorname{sen} \theta e^{i(t+\alpha)} \varphi_{-}^{(1)}+\cos \theta e^{-i(\varphi-\alpha)} \varphi_{-}^{(2)}\right]^{\dagger} \alpha_{1} \dot{\psi}=0
\end{aligned}
$$

Substituindo os resultados das equaçóes $(5.1 .19)$ e $(5.1 .20)$, a equaçâo $(8.1 .2)$ acima se torna:

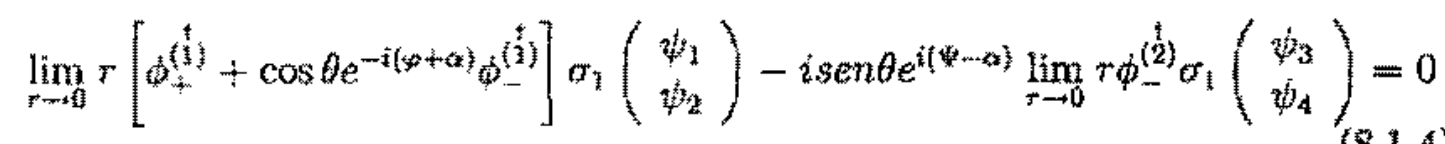


Com os espinores $\phi_{ \pm}^{(1)}$ e $\phi_{ \pm}^{(5)}$ dados pelas equakgoes (5.1.17) e (5.1.18), a equação acima se torna:

$$
\begin{gathered}
\lim _{r \rightarrow 0} r\left[\left(e^{-i \frac{\pi}{4}}-\cos \theta e^{-i(p+\alpha)} e^{i \frac{\pi}{4}}\right) K_{t+1} \psi_{1}+\left(1+\cos \theta e^{-i(p+\alpha)}\right) K_{L} \psi_{2}\right]- \\
-i \operatorname{sen} \theta e^{i(\Psi-\alpha)} \lim _{\tau \rightarrow 0} r\left(-e^{-i \frac{\pi}{4}} K_{3+1+1} \psi_{3}+K_{2} \psi_{4}\right)=0 .
\end{gathered}
$$

Lembrando do comportamento na origem das funcoes $K_{v}$ e $K_{v+1}$ dado pela equação (5.2.6) e usando ats definições te $z, y, z$ e $w$ das equaçoes $(5.4 .4)$ a (5.4.7), a equağ̈o (8.1.5) acima pode ser escrita assim:

$$
\begin{gathered}
\left.\left(e^{-i x}-\cos \theta e^{-i(\varphi+\alpha)} e^{i \frac{\pi}{4}}\right) x+\left(1+\cos \theta e^{-i(\varphi+\alpha)}\right)\right)+ \\
+i \operatorname{sen} \theta e^{i(\Psi-\alpha)} e^{-i \frac{\pi}{4} z}-\sin \theta e^{i(\Psi-\alpha)} w=0
\end{gathered}
$$

Procalendo em relaçäo à exuação $(8.1 .3)$ da mesma que procedemos com a equaço (8.1.2), a equaçăo (8.1.3) pode ser escrita como:

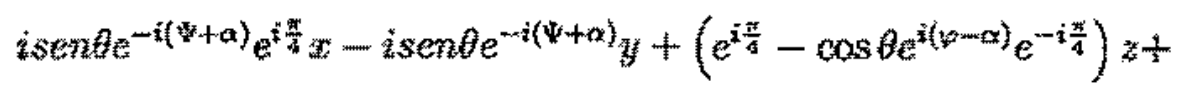

$$
\begin{aligned}
& +\left(1+\cos \theta e^{i(t)-\alpha)}\right) w=0
\end{aligned}
$$

Podemos rescrever as equagoes $(8.1 .5)$ e (8.1.6) assim;

$$
\begin{aligned}
& p x+a y+\operatorname{sen} \theta e^{-i \frac{\pi}{4} z-\operatorname{sen} \theta w}=0 \\
& -\operatorname{sen} \theta e^{i \frac{\pi}{4}} x+\operatorname{sen} \theta y+r z+\sin z=0
\end{aligned}
$$


onde introduzimos as definiçôes:

$$
\begin{aligned}
& p=-i e^{-i(\omega-\alpha)}\left(e^{-i \frac{\pi}{4}}-\cos \theta e^{-i(\varphi+\alpha)} e^{i \frac{\pi}{i}}\right) \\
& q=-i e^{-i(\psi-\alpha)}\left(1+\cos \theta e^{-i(4+\alpha)}\right) \\
& T=i e^{i(\Psi+\alpha)}\left(e^{i \frac{\pi}{4}}-\cos \theta e^{i(t)-\alpha)} e^{-i \frac{\pi}{4}}\right) \\
& s=i e^{i(t+x)}\left(1+\cos \theta e^{i(t+\alpha)}\right) .
\end{aligned}
$$

As equaçöes (8.1.8) e $(8.1 .9)$ representam as condiçôes de contorno a serem satisfaitas pelas auto-funçôs de $H$ na origem, e definem novos domínios $D_{0, \psi_{i}, \alpha},(H)$ onde $H$ é auto-adjunto.

De fato, essas equaçöes fornecem todas as extensóes anto-adjuntas (a quatro parâmetros) para o operador $H$ e evidentemente incluem as dernais condiçöes de contorno apresentadas nos Capítulos 5,6 e 7 e também incluem todas as condigôes de contorno apresentadaş nas teferências $[1-8]$.

\subsection{Determinação das auto-funçōes de $H$ que satisfazem as condiçōes} de contorno mais geraís corn quatro parâmetros.

Na verdade, devemos nos lembrar que o nosso objetivo ao definirmos domínios estendidos para os operadores $H$ e $A$ foi o de verificar a possibilidade de obtermos auto-funçōes simultâneas, $\operatorname{com} H$ e $A$ auto-adjuntos nesses domínios.

No entanto, ju vimos no Capitulo 5 que isso só é possivel para certas combinaçöes lineares das auto-funçöes de $H$, apresentadas na Tabela 4 , sob a condição da equaçio $(5.8 .13)$ ser satisfeitu.

Seja entăo, a auto-funcão de $H$ da equacăo (6.2.1)com energia positiva e com possibilidade de auto-valor da helicidade $\lambda= \pm k$ :

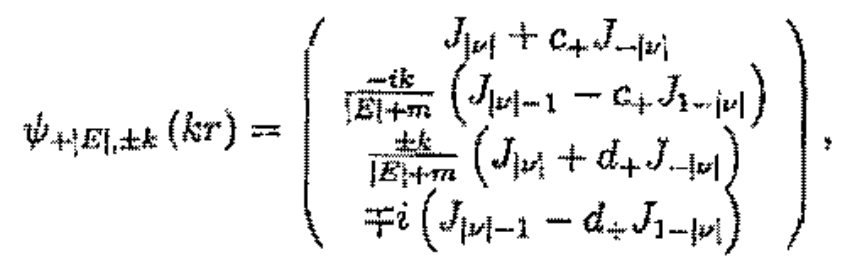

onde tomamos a combinafäo linear (isto $\hat{e}$ as constantes $\alpha \beta$ ) conforme a Tabela 4. 
Vamos agora verificar a passibjlidade das auto-funçües da forma da equaçăo anterior, satisfazendo as condições de contorno das equaçoes (8.1.8) e (8.1.9), satisfazerem também à condicăo da equaçäo (5.8.13), de forma a garantir que tais auto-funçöes de $B$ sejam também auto-funçöes de $\mathrm{A}$.

Lembrando do comportamento na origem das funçoos de Bessel, dado pela equacão (5.2.6) e lembrando das definiçoes de $x, y, z$ e w das equaçoes (5.4.4) a (5.4.7), temos:

$$
\begin{aligned}
& x=(\sqrt{2} m)^{|\omega|-1} k^{-|v|} c_{+}, \\
& y=-i \frac{(\sqrt{2} m)^{-\mid w_{i}} k^{|w|}}{|E|+m} \\
& z= \pm(\sqrt{2} m)^{|\mu|-1} k^{-|\omega|} \frac{k}{|E|+m} d_{*} \\
& w=\mp i(\sqrt{2} m)^{-i v \mid} \frac{k^{|v|}}{k} .
\end{aligned}
$$

Substituindo as expressốes das equaçöes (8.2.2) a (8.2.5) nas equações (8.1.8) e (8.1.9) das condiçôes de contorno, obtemos o seguinte sistema de equaçóes:

$$
\begin{aligned}
& p c_{+}(\sqrt{2 m})^{|\omega|-1} k^{-|\omega|} \pm \operatorname{sen} \theta e^{-i \frac{\pi}{4}} d_{+} \frac{k}{|E|+m}(\sqrt{2} m)^{|\omega|-1} k^{-i m \mid}= \\
& =i(\sqrt{2} m)^{-|\omega|} k^{|v|}\left[\frac{q}{|E|+m} \mp \frac{\operatorname{sen} \theta}{k}\right] \\
& -\operatorname{sen} \theta e^{i \frac{\pi}{2}} c_{+}(\sqrt{2} m)^{|m|-1} k^{-|m|} \pm r d_{+} \frac{k}{|E|+m}(\sqrt{2} m)^{|m| \cdots 1} k^{-|m|}= \\
& =(\sqrt{2} m)^{-|x|} k^{|m|}\left[\frac{\operatorname{sen} \theta}{|E|+m} \pm \frac{s}{k}\right]
\end{aligned}
$$

de onde devemos extrair $c_{+}$e $d_{+}$para depois verificar a possibilidade de satisfação da condiçăo $(5.8 .13)$. 
O sistema acima terá soluçäo apenas se:

$$
\cos \theta \operatorname{sen} \varphi \neq \cos \alpha
$$

Vamos calcular os coeficientes $t_{+}$ed+ através da regra de Cramer. Assim, sendo $\Delta$ o determinante da matriz incompleta do sistema de equaçöes $(8.2 .6)$ e (8.2.7), temos:

$$
\Delta= \pm(\sqrt{2} m)^{2|+| \omega 2} \frac{k^{-2|+|+1}}{|E|+m}\left(e^{2 i \alpha}-2 e^{i \alpha} \cos \theta \operatorname{sen} \varphi+1\right) \text {. }
$$

Vamos introduzir as definisōes:

$$
\begin{gathered}
\gamma_{1}=r q-e^{-i \frac{\pi}{4}} \operatorname{sen}^{2} \theta \\
\gamma_{2}=\left(r+\operatorname{se}^{-i \frac{\pi}{4}}\right) \operatorname{sen} \theta \\
\gamma_{3}=\left(p+q e^{i \frac{\pi}{4}}\right) \operatorname{sen} \theta \\
\gamma_{4}=p s-e^{i \frac{\pi}{4}} \operatorname{sen}^{2} \theta
\end{gathered}
$$

Sendo $\Delta_{1}$ o determinante da matriz obtida de $\Delta$ pela mudança da coluna dos coeficientes de $c_{\leftarrow}$ pela coluna dos termos independentes, temos:

$$
\Delta_{1}= \pm \frac{i}{\sqrt{2} m|E|+m}\left\{\frac{\gamma_{1}}{|E|+m} \mp \frac{\gamma_{2}}{k}\right\}
$$

Sendo $\Delta_{2}$ o deterninante da matriz obtida de $\Delta$ pela mudanga da colnna dos coeficientes de $d_{4}$ pela colma dos termos indapendentes, temos

$$
\Delta_{2}=\frac{i}{\sqrt{2 m}}\left\{\frac{\gamma_{3}}{|E|+m} \pm \frac{\gamma_{4}}{k}\right\}
$$


Substituindo as equasões (8.1.10) a (8.1.13) nas equagōes (8.2.10) a (8.2.13) acima, obtemos:

$$
\begin{gathered}
\gamma_{1}=2 i e^{i \alpha}\left[\operatorname{sen}\left(\alpha+\frac{\pi}{4}\right)+\cos \theta \operatorname{sen}\left(\frac{\pi}{4}-p\right)\right], \\
\gamma_{2}=i \sqrt{2} e^{i(\psi+\alpha)} \operatorname{sen} \theta \\
\gamma_{3}=-i \sqrt{2} e^{-i(\psi-\alpha)} \operatorname{sen} \theta_{1} \\
\gamma_{4}=2 i e^{i \alpha}\left[\operatorname{sen}\left(\alpha-\frac{\pi}{4}\right)-\cos \theta \operatorname{sen}\left(\frac{\pi}{4}-p\right)\right] .
\end{gathered}
$$

Podemos calcular $c_{+}$e $d_{+}$fazendo:

$$
c_{+}=\frac{\Delta_{1}}{\Delta}
$$

e:

$$
d_{+}=\frac{\Delta_{2}}{\Delta} \text {. }
$$

Como queremos que $c_{+}$seja igual $a{ }_{+}$(condiça da equaça (5.8.13)), vamos verificar a possibilidade de:

$$
\Delta_{1}=\Delta_{2 *}
$$

Com $\Delta_{1}$ e $\Delta_{2}$ dados pelas expressōes $(8.2 .14)$ e (8.2.15), a expressăo acima implica em:

$$
\pm \frac{k}{|E|+m}\left\{\frac{\gamma_{1}}{|E|+m} \mp \frac{\gamma_{2}}{k}\right\}=\frac{\gamma_{3}}{|E|+m} \pm \frac{\gamma_{4}}{k}
$$

a qual pode ser escrita como:

$$
\frac{ \pm k^{2} \gamma_{1}-k(|E|+m)\left(\gamma_{3}+\gamma_{4}\right) \pm(|E|+m)^{2} \gamma_{4}}{k(|E|+m)^{2}}=0
$$


Para que a equaça acima seja verificada para qualquer valor de $E$ devemos ter:

$$
\begin{aligned}
& \gamma_{1}=0 \\
& \gamma_{4}=0_{\bar{z}}
\end{aligned}
$$

e:

$$
\gamma_{2}=-\gamma_{3}
$$

Substituindo as equaçoes $(8.2 .16)$ e (8.2.19) nas expresson $(8.2 .25)$ e $(8.2 .26)$ obtemos que:

$$
\alpha=n_{1} \pi \quad, n_{1}=0,1_{1}, 2_{1}, \ldots
$$

e:

$$
\cos \theta(\operatorname{sen} \varphi-\cos \varphi)=(-1)^{n *}
$$

Substituindo a equação (8.2.28) na condição da equação (8.2.8), wsa se torna:

$$
\cos \theta \operatorname{sen} p \neq(-1)^{n_{1}}
$$

Fimalmente, substituindo as duaço $(8.2 .17)$ e $(8.2 .18)$ na expressão $(8.2 .27)$, obtemos que:

$$
\Psi=n_{2} \pi \quad, n_{2}=0,1,2, \ldots
$$

Assim a imposiçäo da condiçäo da equaçẫo (5.8.13) para obtenção de autofunçós comuns no domínio $D_{0, w_{1}, \alpha}(H)$ condu as relaços entre os parâmetros da extensăo dadas pelas equagếes $(8.2 .28)$ a $(8.2 .31)$, esquematizadas na tabela abaixo.

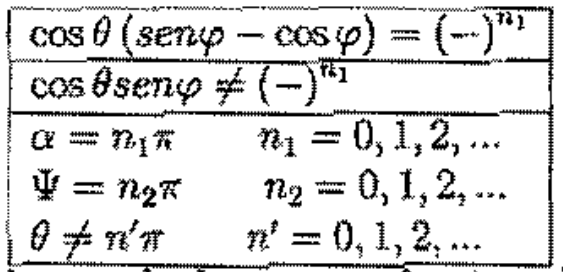

Tabela 8 - Relacóces que devem obedecer os parâmetros da extensäo auto-adjunta mais geral de $H$ para obtençäo de auto-funçoses comus aos operadores $H \in \Lambda$, 
A viltima condição da Tabela 8 foi colocada para que sejam obtidas soluçōes diferentes das encontradas na Sexäo 5.9 .

Dessa forma, para uma auto-hunçăo de $H$ da forma da equagăo $(8.2 .1)$ e com os parâmetros da extensāo satisfazendo as condiçós da Tabela 8, possível obter auto-funçôes simultâneas para $H$ e $\Lambda$, uma vez que as condiçờ apresentadas na Tabela 8, implicam na satisfaça da equação (5.8.13).

Com condiçoes da Tabela 8, temcr que as equaçôes $(8.2 .25)$ a $(8.2 .26)$ fornecem:

$$
\begin{gathered}
\gamma_{1}=0 \\
\gamma_{4}=0 \\
\gamma_{2}=-\gamma_{3}=i \sqrt{2}(--)^{n_{1}+n_{2}} \operatorname{sen} \theta
\end{gathered}
$$

De modo que, assim, obtemos $\Delta_{1}, \Delta_{2}$ e $\Delta$ dados pelas equagöes $(8.2 .9),(8.2 .14)$ e (8.2.15) como sendo:

$$
\Delta_{1}=\Delta_{2}=\frac{(-)^{n_{1}+n_{2}} \operatorname{sen} \theta}{m(|E|+m)}
$$

e:

$$
\Delta= \pm(\sqrt{2} m)^{2|\nu|-2} \frac{k}{|E|+m} 2\left(1-(-)^{n+1} \cos \theta \operatorname{sen} \varphi\right)
$$

Com os resultados das equagoes acima podemos obter $c_{4}$ e $d_{4}$ dados por $(8.2 .20)$ e (8.2.21) como sendo:

$$
c_{+}=d_{+}=\frac{\sqrt{2}}{2} \frac{(-)^{n_{1}+x_{2}} \operatorname{sen} \theta}{\left(1-(-)^{n_{1}} \cos \theta \operatorname{sen} \varphi\right)}\left(\frac{k}{\sqrt{2} m}\right)^{2|\varphi|-1}
$$

para $\lambda=+k$ e

$$
c_{+}=d_{+}=-\frac{\sqrt{2}}{2} \frac{(-m)^{n_{1}+n_{2}} \operatorname{sen} \theta}{\left(1-(-)^{n_{1}} \cos \theta \operatorname{sen} \varphi\right)}\left(\frac{k}{\sqrt{2} m}\right)^{2, n-1}
$$

para $\lambda=-k$ 
Observando a forma das auto-funcoes de $H$ dada polas equaços (5.5.1) a (5.5.5) e a expressão dos coeficientes $\alpha$ e $\beta$ (Tabela 4), vemos que para obter as auto-funçous simultâneas de $H$ e $A$ com energia negativa, basta trocar $|E| \rightarrow$ $-|E|$.

Desse modo, considerando os resultados obtidos nessa segâo teremos se sezuntes auto-funços simultâneas para $H$ \& $A$, apresentadas na Tabela 9 rbaixo e com os parâmetros da extensäo relacionados conforme mostra a Tabela 8 acima:

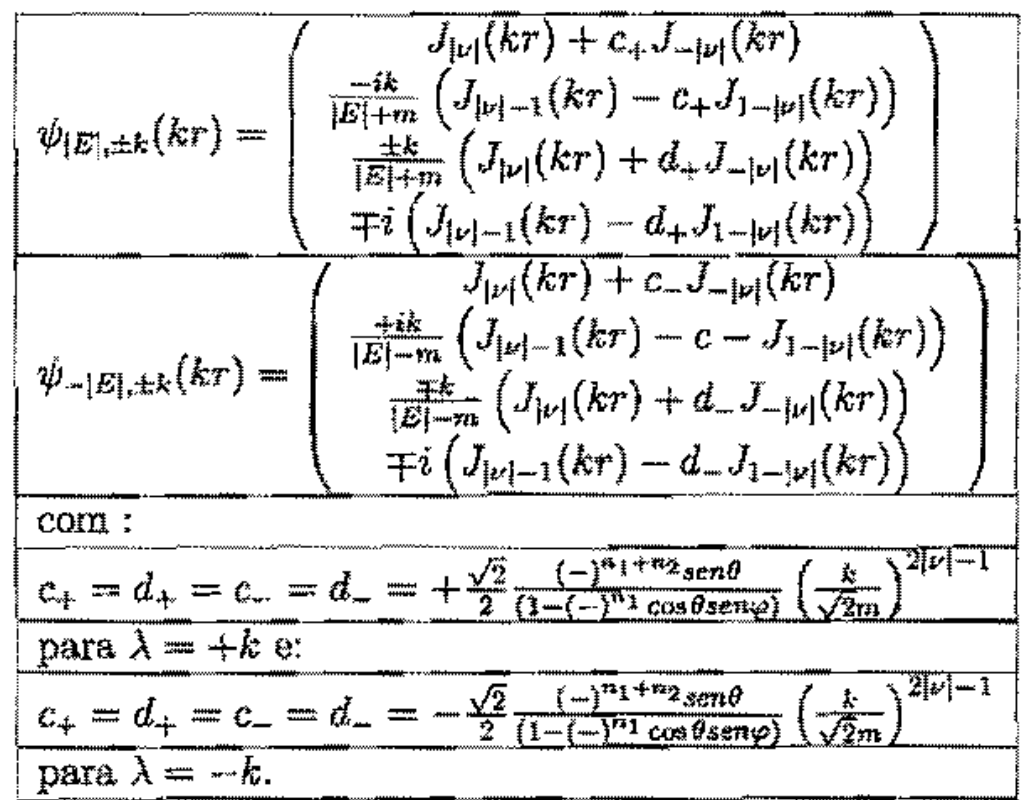

Tabela 9 - Auto-funcós simultaneas para $H$ e $\Lambda$ no domínio mais geral onde o operador hamiltoniana canto-adjunto.

As auto-funçoes da Tabela 9 são auto-funçöes simultaneas dos operadores $H$ e $A$, no domínio mais geral possivel $D_{0, \Psi_{1}, \alpha}(H)$ onde $H$ é auto-adjunto, definido pelas equacotes $(8.1 .8)$ e (8.1.9) e com os parametros da extensão satisfazendo as condicoes da Tabela 8.

Surge, entän, novamente, a questào crucial:

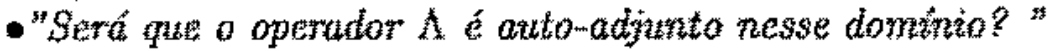


8.3. Verificando se $A$ auto-adjunto no domínio mais geral $D_{\Psi_{1}, \alpha_{1}, p}^{t}(H)$ encontrado.

Vamos verificar se $\Lambda \odot$ antomadjunto no dominio mais geral $D_{\Psi, \alpha, b, t}^{\prime}(h)$ definido pelas equaços (8.1.8) e (8.1.9) com as condiçöes da Tabela 8 .

Jâ vimos no Capitulo 5, que as condiçoes de contorno mais gerais a serem satisfeitas pelas auto-funçós de $\Lambda$ na origem, paranetrizadas pela matriz $V$ unitária, sấo dadas pelas equaços $(5.3 .11)$ e (5.3.12) abaixo transcritas e correspondern a todas as possiveis extençoes anto-adjuntas de $\mathrm{A}$ :

$\left(1+\bar{v}_{11}+i \bar{w}_{12}\right) w+\left(1-\bar{v}_{11}+i \bar{w}_{12}\right) z+\left(1+\bar{v}_{11}-\bar{w}_{12}\right) y+\left(1-\bar{v}_{11}-i \bar{v}_{12}\right) x=0$

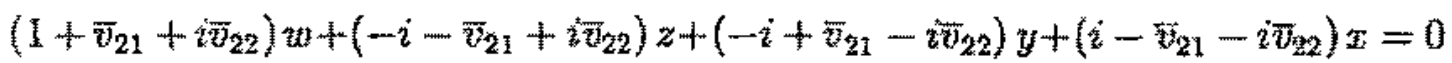

Eossivel verificar que as equagóes (8.1.10) e (8.1.11), que definem o domínio mais geral $D_{f, \psi, \psi, \alpha, \alpha}^{i}(H)$ corn os parâmetros da extensäo satisfazendo as condiçōes da Tabela 8, podem ser escritas como:

$$
\begin{aligned}
& x=m_{0} \operatorname{sen} \varphi x, \\
& y=m_{0} \cos \varphi z
\end{aligned}
$$

onde:

$$
m_{\theta}=\sqrt[5]{2}(-)^{n_{2}} \cot \theta
$$

Queremos definir $\Lambda$ no domínio $D_{\theta, t_{,}, \alpha_{f}}^{\prime}(H)$ definido pelas equaçöes acima (com os parâmetros $\theta$ e $\varphi$ satisfazendo as condiçoos das equaços (8.2.29) e (8.2.30)) e verificar se ele é auto-adjunto nesse dominio.

Como o sistema de equaçoes $(8.3 .3)$ e $(8.3 .4)$ é o mesmo que o encontrado na Seçă 7.6 do Capitulo 7 (equacios $(7.2 .7)$ e $(7.2 .8)$ ), podemos, da mesma form 8 que naquela seção, concluir que $\Lambda$ é auto-adjunto nos domínios definido pelas exuaçôes $(8.3 .3)$ e $(8.3,4)$ sob as condiçōes da Tabela 8 . 
Portanto, $H$ e $A$ comutam e consequentemente admitem auto-funçoes simultâneas (Tabela 9) no domina mais geral definido por (8.3.3) e (8.3.4) sob as condiçöes ta Tabela 8 , onde ambos os operadores $H$ e $\Lambda$, säo auto-adjuntos. Em outras patavras, as dinâmicas definidas através das enusçóes (8.3.9) e (8.3.4) com as condiçöes da Tabela 8 conservam a helicidade.

É muito fácil verificar que as condişöes de contorno obtidas no Capítulo 7 , atravếs das equaçōes (7.1.12) e (7.1.13) com as condiçōes da Tabela 5, sâo um caso particular das condiçôs de contorno obtidas nesse capítulo através das equações (8.1.8) e (8.1.9) com as condicões da Tabela 8 (basta tomar, por exemplo $\alpha=0$ na Tabela 8 para obter as equações encontradas no Capittulo 7). 


\section{Capítulo 9}

\section{Simetria de Aharonov-Bohm e conservação da helicidade}

Nesse capítulo vamos analisar a possibilidade de preservacão da Simetria $\phi \rightarrow \phi+1$ para as dinârnicas definidas pelas condiçótes de contorno dos Capitulos 7 e 8 que conservarn a helicidade.

Uma peculiaridade muito înteressante das condiçōes de contorno obtidas nos Capítulos 7 e 8 anteriores é que essas condigöes além de permitirema a conservação da helicidade preservam também outra importante simetria do hamiltoniana: a simetria de Aharonov Bohm $(\phi \rightarrow \phi+1)$, como discutiremos a seguir. A existência de dinầnicas onde ambas as simetrias são preservadas não ocorre para nenhuma das dinâmicas definidas pelas condiçōes de contorno oferecidas pela literatura, entre elas [1],\{2],\{3],[4],[5],[6] e [7] para as quais a preservação de uma simetria implic na quebra da outra (faremos uma discussão sobre o conteúdo dessas referêtecias no Capitulo 10 ).

De fato, nos Capitulos 7 e 8 foram encontradas famílias de extensōes autoadjuntas, para as quais os operadores $H$ e $A$ além de comutarem entre si ( e consequentemente admitirem auto-funçōes comuns), preservam tarnbém a sirnetria $\phi \rightarrow \phi+1$ do hamiltoniana. Para vermos como isso acontece, prexisamos analisar se há continuidade na dinâmic (isto é, das condiçôes de contorno e das auto-funçöes ) como funçầo do Auxo $\phi$. Precisamos também analisar se há ou năo algum problema como o que ocorria para as condiçóes de contorno das referências [2] e [4] quando o fluxo magnético $\phi \rightarrow \phi+k$ trocando de sinal, devido a assimetria existente entre os gráficos do parâmetro da extensão como função do fluxo magnético $\phi$ nos casos em que $n>0$ e $n<0$. Para isso vamos considerar as condiçós de contorno do Capítulo 8 que são as mais gerais (as condiçóes de contorno do capítulo 7 säo un caso particilar dessas condiçoes de contorno), dadas pelas equaçoes (8.1.8) e (8.1.9), sujeitas às condiçōes da Tabela 8 , abaixo transcritas:

$$
\begin{aligned}
& p x+q y+\operatorname{sen} \theta e^{-i \frac{x}{4}} z-\operatorname{sen} \theta w=0 \\
& -\operatorname{sen} \theta e^{i \frac{\pi}{4}} x+\operatorname{sen} \theta y+r z+\sin w=0
\end{aligned}
$$


onde:

$$
\begin{gathered}
p=\sigma^{*}=-i(-)^{n_{2}+n_{1}}\left(e^{-i \frac{\pi}{4}}-(-)^{n_{1}} \cos \theta e^{-i \varphi} e^{i \frac{\pi}{4}}\right) \\
q=s^{*}=-i(-)^{n_{2}+n_{i}}\left(1+(-)^{n_{1}} \cos e^{-i \varphi}\right),
\end{gathered}
$$

$\operatorname{com}$ os parâmetros $\theta$ e $\varphi$ satisfazendo ès condiç̧̄es da Tabela 8 que representam as condiçöes para conservaç̄o da helicidade, abaixo transcritas:

$$
\begin{gathered}
\cos \theta \operatorname{sen}\left(\varphi-\frac{\pi}{4}\right)=(-)^{n_{1}} \frac{1}{\sqrt{2}} \\
\cos \theta \operatorname{sen} \varphi \neq(-)^{n_{2}} .
\end{gathered}
$$

Na verdade, apenas a equaçäo (9.5) expressia a condiçäo de conservaça da helioidade, pois a equação (9.6) garante apenas que as expressồ parc as constantes $c_{ \pm}$e $d_{ \pm}$(da Tabela 9) tenham denominador diferente de zero.

Devernos observar que as condiçós $(9.5)$ e (9.6) impöem, por sua vez, certas restriçoes wos possiveis valores dos parâmetros $\theta$ e $\varphi$, os quais, a princípio, poderiam variar cada um independentemente no intervalo de 0 a $2 \pi$.

A equarão (9.5) pode ser escrita sob a forma:

$$
\theta=\arccos \left\{(-)^{n} \frac{1}{\sqrt{2}} \operatorname{cosec}\left(\varphi-\frac{\pi}{4}\right)\right\}
$$

de onde vemos que, para $n_{1}$ par 0 intervalo de variaça de $\varphi$ fica restrito ao intervalo $\left[\frac{\pi}{2}, \pi\right]$, o que corresponde a $\theta$ variando no intervalo $\left[0, \frac{\pi}{4}\right]$, e $\varphi$ variando no intervalo $\left[\frac{3 \pi}{2}, 2 \pi\right]$ com $\theta$ variando no intervalo $\left[\frac{3 \pi}{4}, \pi\right]$. Fora desses intervalos a equaģào (9.7) nâo pode ser verificada. Veja o gráfico da figura 1. Para $n_{1}$ ímpar 0 intervalo de $\rho$ fica restrito a $\left[\frac{\pi}{2}, \pi\right]$ com $\theta$ no intervalo $\left[\frac{3 \pi}{4}, \pi\right]$ e $\varphi$ no intervalo $\left[\frac{3 \pi}{2}, 2 \pi\right] \operatorname{com} \theta$ no intervalo $\left[0, \frac{\pi}{4}\right]$. Veja também o gráfico da figura 3.

Por outro lado, a condicio (9.6) impede que tenhamos para $n_{1}$ par $\theta=0,2 \pi$, simultaneamente com $\varphi=\frac{\pi}{2}$ e que tenhamos $\theta=\pi$ simultanearmente $\operatorname{com} \varphi=\frac{3 \pi}{2}$. Para $n_{1}$ ímpar a condigäo $(9.6)$ impede que tenhamos $\theta=0,2 \pi$ com $\varphi=\frac{3 \pi}{2}$ $\theta=\pi \operatorname{com} p=\frac{\pi}{2}$. 
Todos esses resultados estäo apresentados na Tabela 10 abaixo e obviamente se referem ao caso do intervalo crítico $-1<v<0$ (on seja, $-n-1<\phi<-n$ ) para o qual foram feitas as extensöes anto-adjuntas:

\begin{tabular}{|c|c|}
\hline \multicolumn{2}{|c|}{ Para $\nu:]-1,0[$, i.e. $, \phi:]-n-1,-1$} \\
\hline \begin{tabular}{l|l}
$\varphi:\left[\frac{\pi}{2}, \pi\right]$ \\
$\left.\varphi: \frac{3 \pi}{2}, 2 \pi\right]$
\end{tabular} & $\begin{array}{ll}\longrightarrow & \theta:\left[0, \frac{\pi}{4}\right] \\
\longrightarrow & \theta:\left[\frac{3 \pi}{4}, \pi\right]\end{array}$ \\
\hline \multicolumn{2}{|c|}{-Caso $n_{1}=$ ímpar } \\
\hline $\begin{array}{l}\varphi:\left[\frac{\pi}{2}, \pi\right] \\
\varphi:\left[\frac{3 \pi}{2}, 2 \pi\right]\end{array}$ & $\begin{array}{ll}\longrightarrow & \theta:\left[0, \frac{\pi}{4}\right] \\
\longrightarrow & \theta:\left[\frac{3 \pi}{4}, \pi\right]\end{array}$ \\
\hline
\end{tabular}

Tabela 10 - Intervalos de variação dos parẫmetros da extensão na condição de conservação da helicidade.

Para o caso $\nu \geq 0$ (ou seja $\phi \geq-n$ para certo valor do momento angular $n$ fixo), sabemos que a única soluçāo adraissivel (por ser de quadrado integrável na vizinhanģa da origem) é a soluçäo regular na origem o que significa que na equação (2.2.31) apenas a soluçăo $J_{v}$ deve sobreviver na primeira componente da funçào de onda (e apenas a solução $J_{v+1}$ deve sobreviver na segunda componente de função de onda).

Pata que isso aconteça as exuaçōes (9.1) e (9.2) das condições de contorno devem fornecer $c_{ \pm}=t_{ \pm}=\infty$, o que significa que devemos ter, naquelas equaçōes:

$$
\begin{gathered}
e^{i \frac{\pi}{x}} \operatorname{sen} \theta=0 \\
p=r^{*}=0
\end{gathered}
$$

e:

$$
q=s^{*} \neq 0 .
$$

Isso ocorre para os seguintes valores dos parâmetros:

$$
\theta=0,2 \pi \quad \text { com } \quad \varphi=\frac{\pi}{2}
$$

oul:

$$
\theta=\pi \quad \operatorname{cotn} \quad \varphi=\frac{3 \pi}{2} .
$$


para $n_{1}$ escolhido par e:

$$
\theta=0,2 \pi \quad \operatorname{com} \quad \phi=\frac{3 \pi}{2}
$$

ou:

$$
\theta=\pi \quad \operatorname{com} \quad \varphi=\frac{\pi}{2}
$$

para na escolhido ímpar e com os parâmetros $\theta$ e $\varphi$ satisfazendo a equação (9.5).

Para o caso $\nu \leq-1$ (ou seja $\phi \leq-n-1$ para certo valor do movimento angular $n$ fixo), devemos novamente ter que a única solução admissível é a soluçäo regular na origem, o que significa que na equasăo (2.2.31) apenas a solução $I_{\text {- }}$ deve sobreviver na primeira componente da funça de onda (e apenas a solução $J_{-\nu-1}$ deve sobreviver na segunda componente da função de onda).

Para que isso ocorra as equacoes $(9.1)$ e $(9.2)$ das condiçoes de contorno devem fornecer $c_{-}=d_{i}=0,0$ que evidentemente ocorre se:

$$
\theta=0,2 \pi \quad \text { com } \quad \varphi=\pi
$$

ci:

$$
\theta=\pi \quad \text { com } \quad \varphi=0,2 \pi
$$

para n par

$$
\theta=0,2 \pi \quad \operatorname{com} \quad \varphi=0,2 \pi
$$

ol:

$$
\theta=\pi \quad \operatorname{com} \quad \varphi=\pi
$$

para $n_{1}$ impar, com os parâmetros $\theta$ e $\varphi$ salisfazendo a equaçấo (9.5). 
Esses resultudos estão apresentados na Tabela 11:

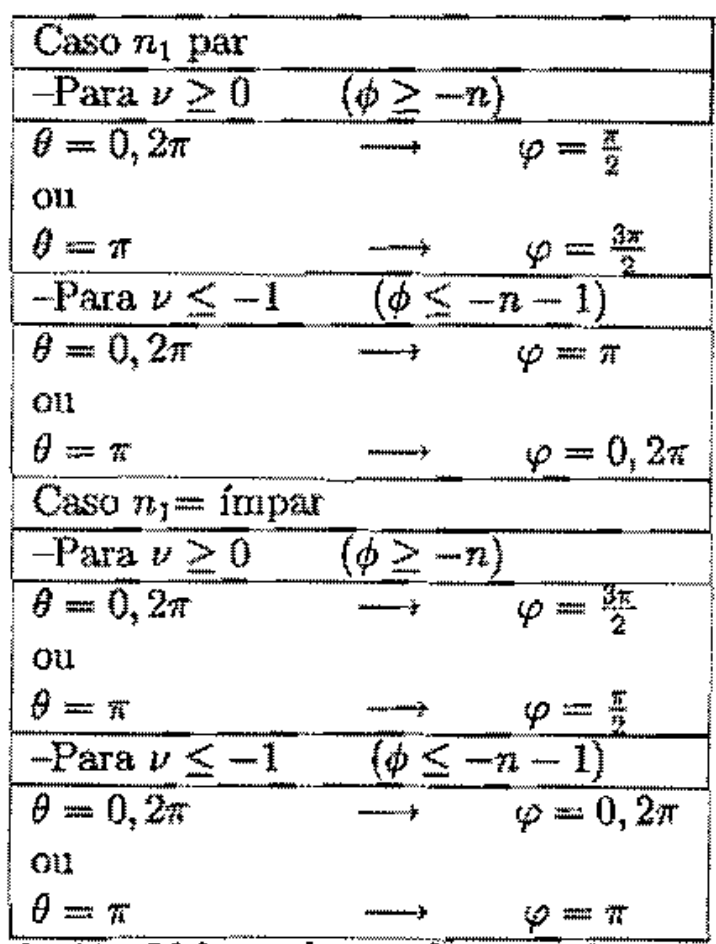

Tabela 11 - Valores dos parâmetros da extensão para os casos $y \geq 0$ e $v \leq-1$.

Analisando os resultados das Tabelas 10 e 11 , vemos que para certas escolhas dos parâmetros 0 e $\varphi$ é possivel obter, atraves de interpolação, a contizuidade da dinåmica como função do fluxo $\phi$ e a preservagäo da simetria $\phi \rightarrow \phi+1$, simultaneamente com a condiçâo de conservagäo da helicidade da equaçà (9.5) satisfeita.

Para $n_{1}$ par, por exemplo, podemoz tomar:

$$
\theta(\phi)=0, \text { wara } \phi \geq-n
$$

com:

$$
p(\phi)=\frac{*}{2}, \text { para } \phi \geq-n
$$


e:

$$
\theta(\phi)=0, \text { para } \phi \leq-n-1
$$

com:

$$
\varphi(\phi)=\pi, \text { para } \phi \leq-m-1 \text {. }
$$

Para um dado valor do momento angular ne (positivo ou negatiwo), vemos que para garantir a continuidade da dinâmica (isto ce das condiçôes de contorno e das funçoes de onda) como funçäo do tuxo $\phi$ e a preservação da simetria $\phi \rightarrow \phi+1$; podemos interpolar as funçöes:

$$
\begin{gathered}
\theta(\phi)=0, \text { para } \phi \geq-n \\
\theta(\phi)=0, \text { para } \phi \leq-n-1
\end{gathered}
$$

e:

$$
\begin{gathered}
\varphi(\phi)=\frac{\pi}{2}, \text { para } \phi \geq-n \\
\varphi(\phi)=\pi, \text { para } \phi \leq-n-1
\end{gathered}
$$

no intervalo $-n-1<\phi<-n$, garantindo que a condiçäo de conservaçäo da helicidade da equacäo (9.5) seja satisfeita. Note que isso é possivel para essas intervalos de variaçäo dos parâmetros (veja a Tabela 10).

Assim, vamos tomar:

$$
\varphi(\phi)=f(\phi+n+1),-n-1<\phi<-n,
$$

onde $f$ e uma funça cuja forma independe de $n$ e contínua no intervalo $[0,1]$ e tal que: $f(0)=\pi$ e $f(1)=\frac{*}{2}$. É crucial que para esse intervalo de variacão do parâmetro $\varphi$ é possivel garantir a satisfacầ da equagăo (9.5) com:

$$
\theta(\varphi(\phi))=\operatorname{arccss}\left\{\frac{1}{\sqrt{2}} \operatorname{cosec}\left(f(\phi+n+1)-\frac{\pi}{4}\right)\right\},-n-1<\phi<-n
$$

$\operatorname{com} \theta(\phi(\phi))$ variando no intervalo $\left[0, \frac{\pi}{4}\right]$ (de modo que $\theta(\phi(\phi)$ ) 6 uma função contínua). Veja os gráficos das figuras $2 \mathrm{a}, 2 \mathrm{~b}$ e 1 . 
Note também que para $n>0$ (movimento angular positivo) os gráficos das figuras $2 \mathrm{a}$ a $2 \mathrm{~b}$ terão as mesmas formas, nüo existindo portanto qualquer assimetria entre os casos $n>0$ e $n<0$, contrariamente as que ocorre nos casos das referências [2], 44$]$.

Uma outra possivel escolha para os valores dos parâmetros para o caso de $n_{1}$ ser par e mosirada nas figuras $2 \mathrm{c}$ e $2 \mathrm{~d}$. Note que nesse caso também é possivel a interpolagão (que garante a presservaçäo da simetria $\phi \rightarrow \phi+1$ e a conkinuidade da dinânica como função de 申) com a condiçâo da equaça (9.5) de conservaçăo da helicidade satisfeita.

Para o caso de $n_{1}$ inpar, podemos tomar:

$$
\theta(\phi)=0, \text { para } \phi \geq-n
$$

com:

e:

$$
\phi(\phi)=\frac{3 \pi}{2} \text { para } \phi \geq-n
$$

$$
\theta(\phi)=0 \text { para } \phi \leq-n-1
$$

com:

$$
\varphi(\phi)=2 m \text { para } \leq \leq-n-1 \text {. }
$$

Para um dado valor do momento zungular $n$ (positivo ou negativo), a fim de garantirmos a continutade das fungöes de onda como funça de 4 e a preservaçäo da simetria $\phi \rightarrow \phi+1$, vamos interpolar as funçoes:

$$
\begin{gathered}
\theta(\phi)=0 \text { para } \phi \geq-n \\
\theta(\phi)=0 \text { para } \phi \leq-n-1
\end{gathered}
$$

e:

$$
\begin{gathered}
\phi(\phi)=\frac{3 \pi}{2} \text { para } \phi \geq-n \\
\varphi(\phi)=2 \text { para } \phi \leq-n-1,
\end{gathered}
$$

no intervalo $-n-1<\phi<-n$, garantindo que a condigà de conservacăo da helieidade da equação (9.5) seja satisfeita. Note que isso é possível para esse intervalo de variação dos parâmetros (veja a Tabela 10). 
Assim, vamos tomat:

$$
\varphi(\phi)=g(\phi+n+1),-n-1<\phi<-n
$$

onde $g$ é uma função cuja forma independe de $n$ e continua no intervalo $[0,1]$ 。 tal que: $g(0)=2 \pi$ e $g(1)=\frac{3 \pi}{2}$. Novamente, erucial que para esse intervalo de variaçäo do parametro $\varphi$ é possivel garantir a satisfaça da da exuagio (9.5) com:

$$
\theta(\varphi(\phi))=\arccos \left\{-\frac{1}{\sqrt{2}}\left(g(\phi+n+1)-\frac{\pi}{4}\right)\right\}, \quad, \quad-n-1<\phi<-n
$$

$\operatorname{com} \theta(\varphi(\phi))$ variando no intervalo $\left[0, \frac{\pi}{4}\right]$ (de modo que $\theta(\varphi(\phi))$ t una funcão contínua). Veja as gráficos das figuras $4 \mathrm{a}, 4 \mathrm{~b}$ e 3 .

Note que as formas dos gráficos das figuras 4a e Al serāo as mesmas para o caso em que o momento angular $n$ é positivo, nầo existindo portanto qualquer assimetria entre os casos $n>0$ e $n<0$.

Uma outra possivel escolha para os valores dos parâmetros para o caso da escolha de $n_{1}$ ser ímpar 6 mostrada nes figuras $4 c$ e $4 d$. Note que nesse caso tambén é possivel a interpolação (que garante a continutdade da dinämica como funça do fluxo e a preservaça da simetria $\phi \rightarrow \phi+1) \mathrm{com}$ a condiçäo (9.5) de conservaça ta helicidade satisfeita.

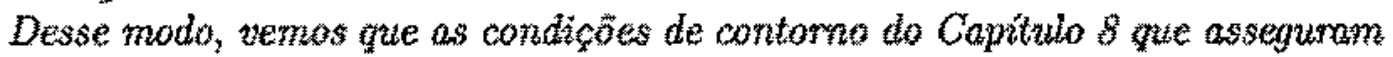
tanamicas onde a helicidade econservada, também podem, parto certas escollas dos parämetros discutidas nesse caphalu, preservar a simetria $\phi \rightarrow \phi+1$, havendo tambén continuidade das dinânicas (das condigóes de contorno e das funcöes de onda) como hunção do fizuro magnético $\phi$, o que näo se conseguia atruếs das demais dintânicas encontradas na literatura $[1],[2],[3],[4], 15],(6]$ e $[7]$. 


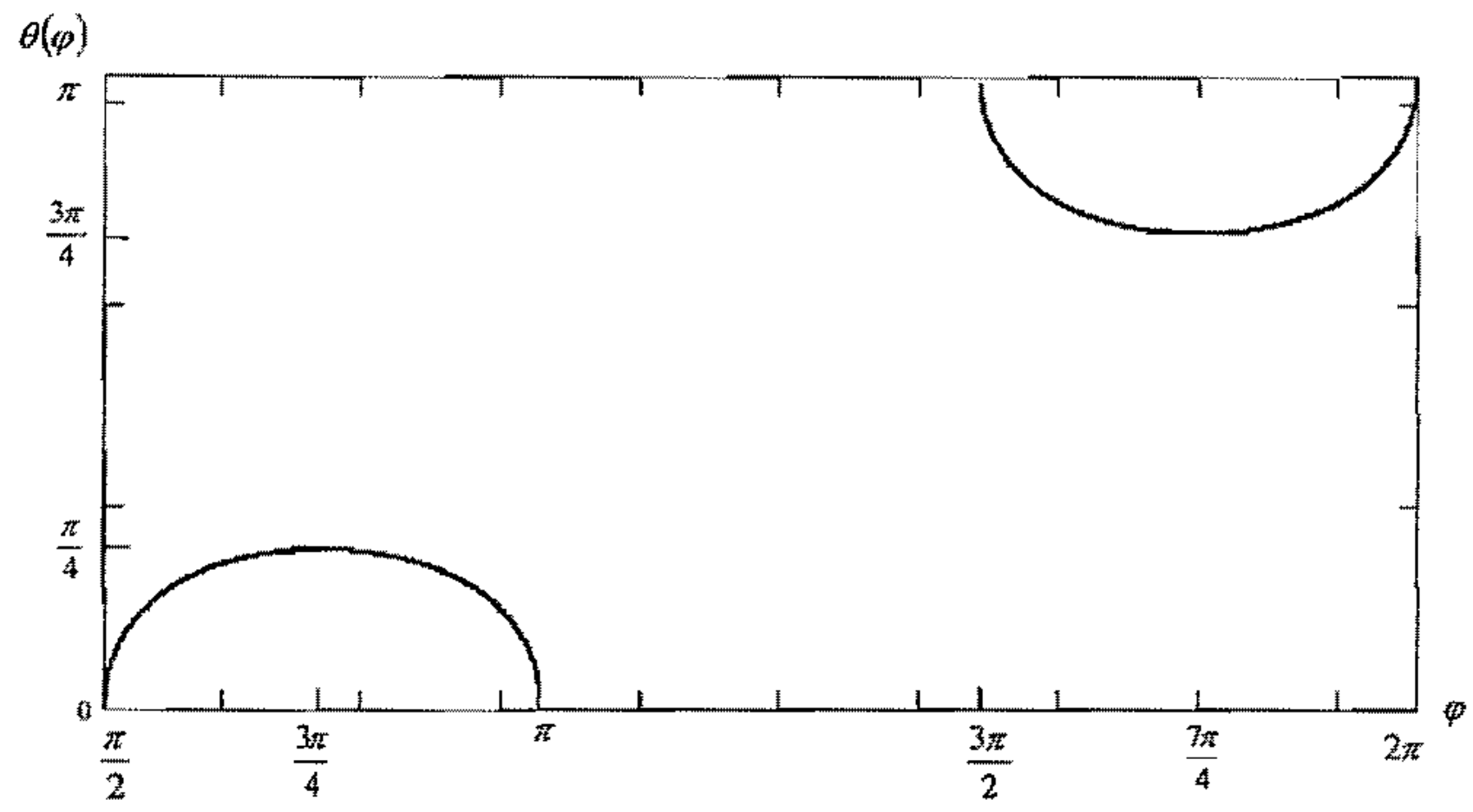

Figura 1- Este gráfico mostra a dependência entre os parâmetros $\theta$ e $\varphi$ que garante a conservação da helicidade quando $n_{\uparrow}$ é par. 


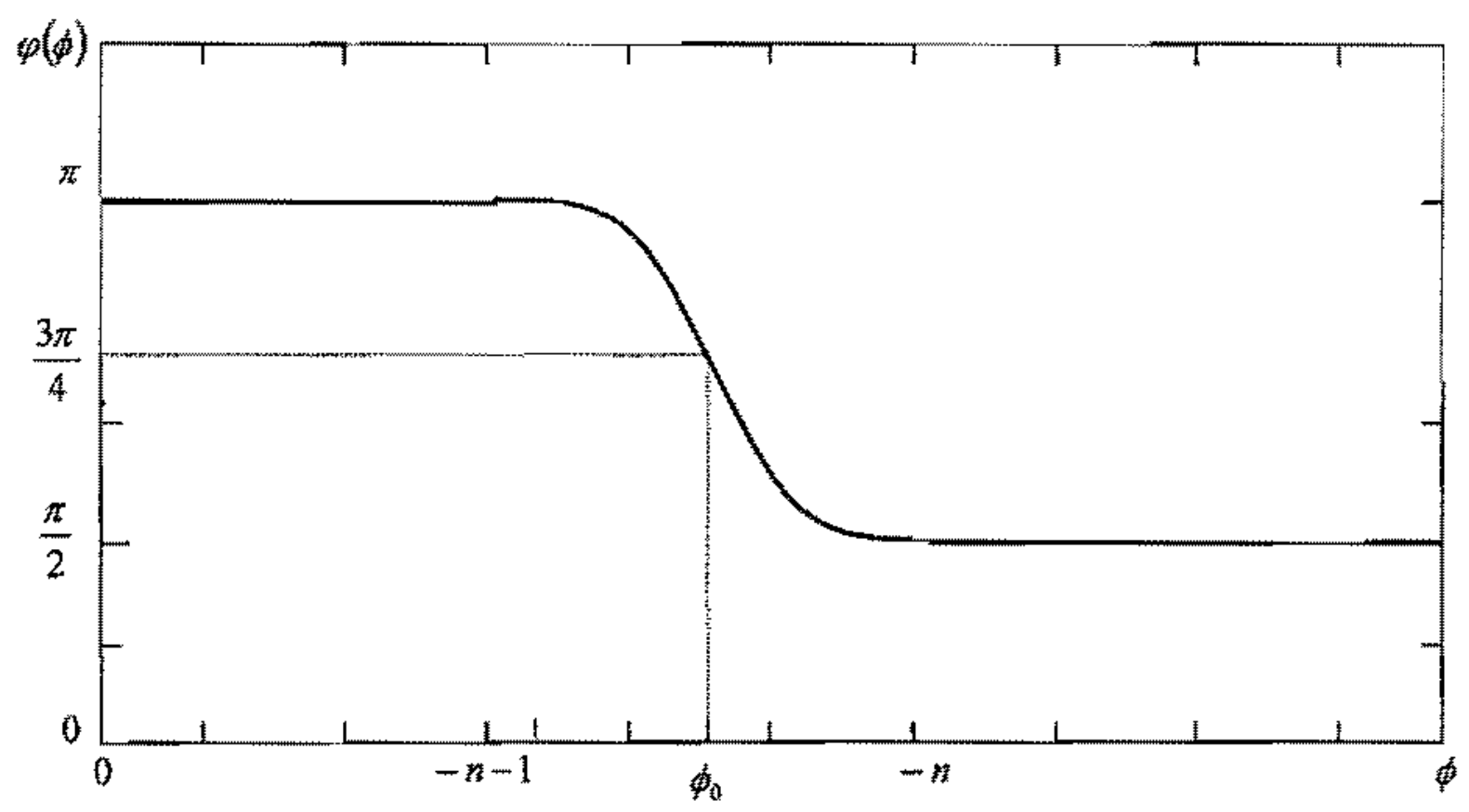

Figura 2a - Interpolação com conservação da helicidade para o parâmetro $\varphi$ como funçẵo do fluxo magnético $\$$ (para momento angular negativo $n$ ) quando $n_{4}$ é par. 


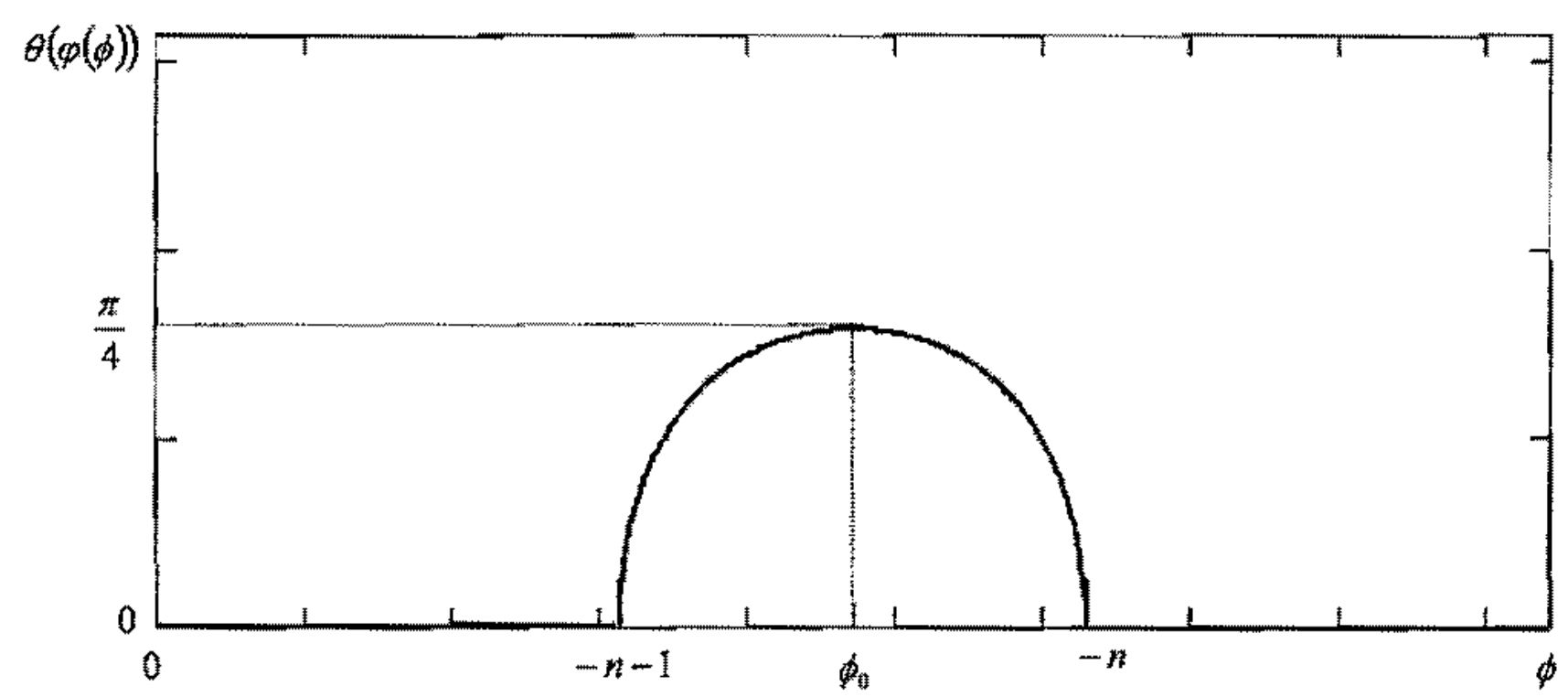

Figura $2 b$-Interpolação com conservação da helicidade para o parâmetro $\theta$ como função do fluxo magnético $\phi$ (para momento angular negativo n) quando nłé par. 


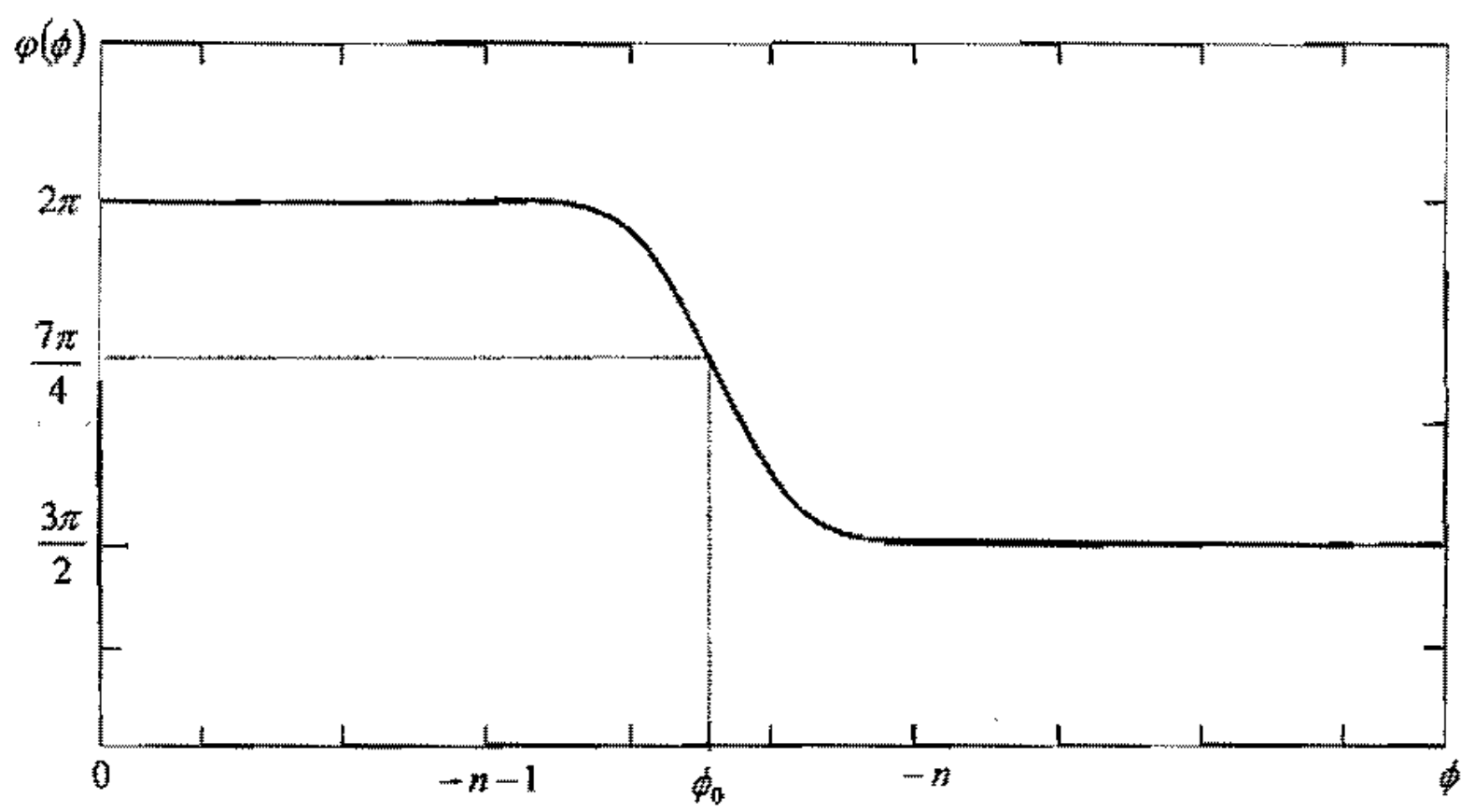

Figura $2 \mathrm{c}$ - Interpolação com conservaçằo da helicidade para o parâmetró $\varphi$ como função do fluxo magnético $\phi$ (para momento angular negativo n) quando né par. 


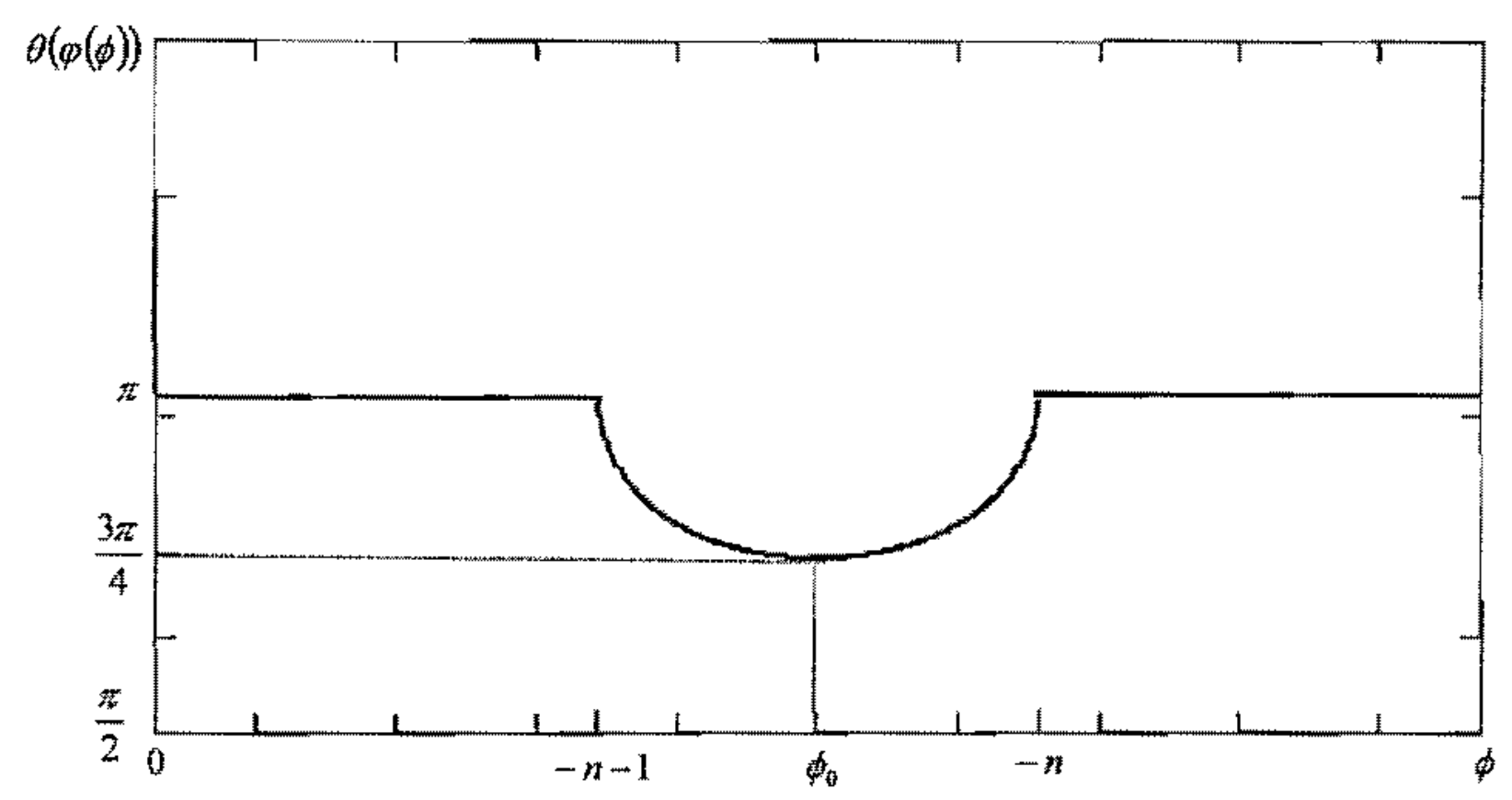

Figura 2d - Intexpolação com conservação da helicidade para o parâmetro $\theta$ como função do fluxo magnético $\phi$ (para momento angular negativo n) quando né par. 


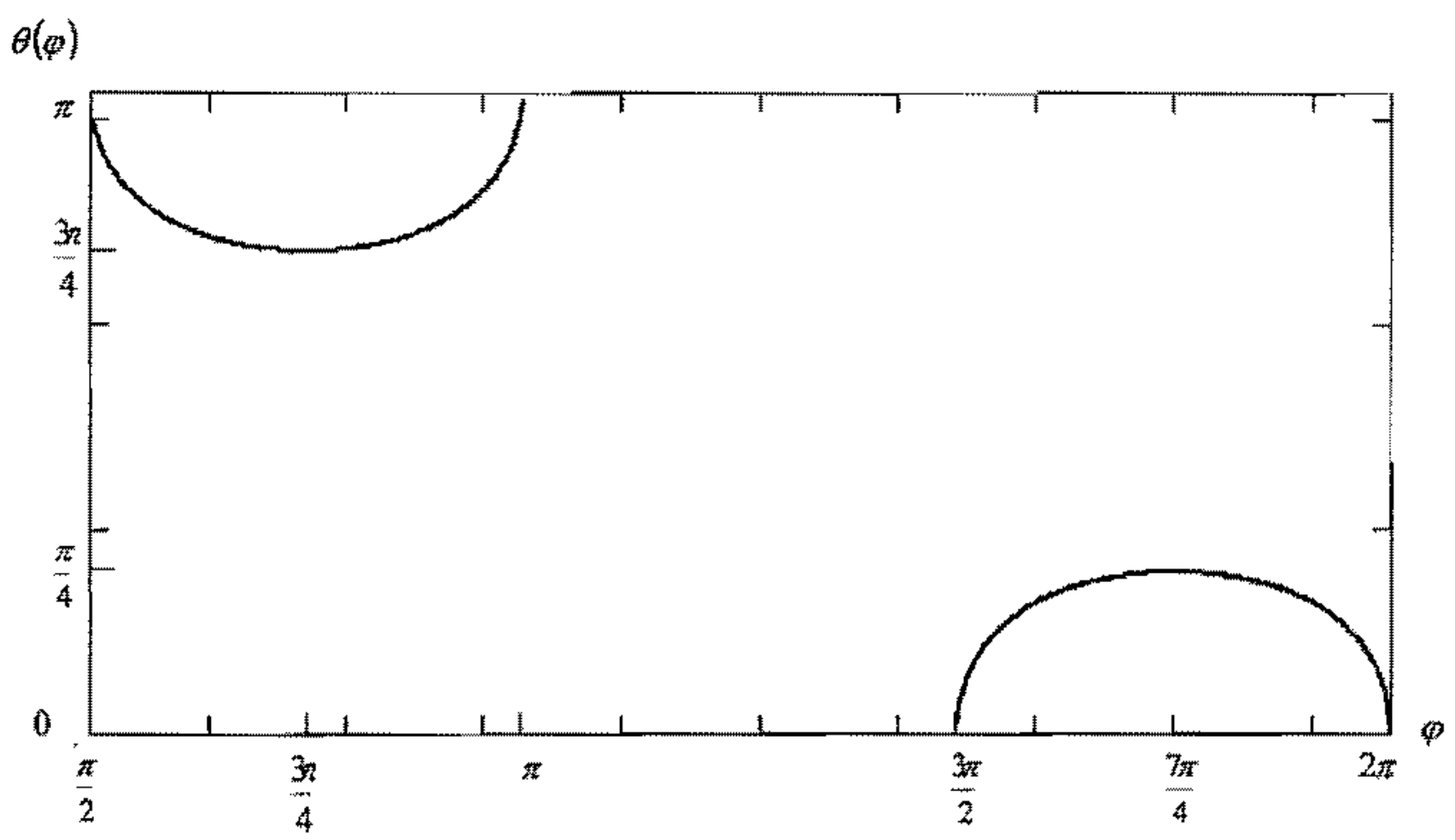

Figura 3 - Este gráfico mostra a dependẻncia entre os parâmetros $\theta$ e $\varphi$ que garante a conservação da helicidade quando $n_{4}$ é ímpar. 


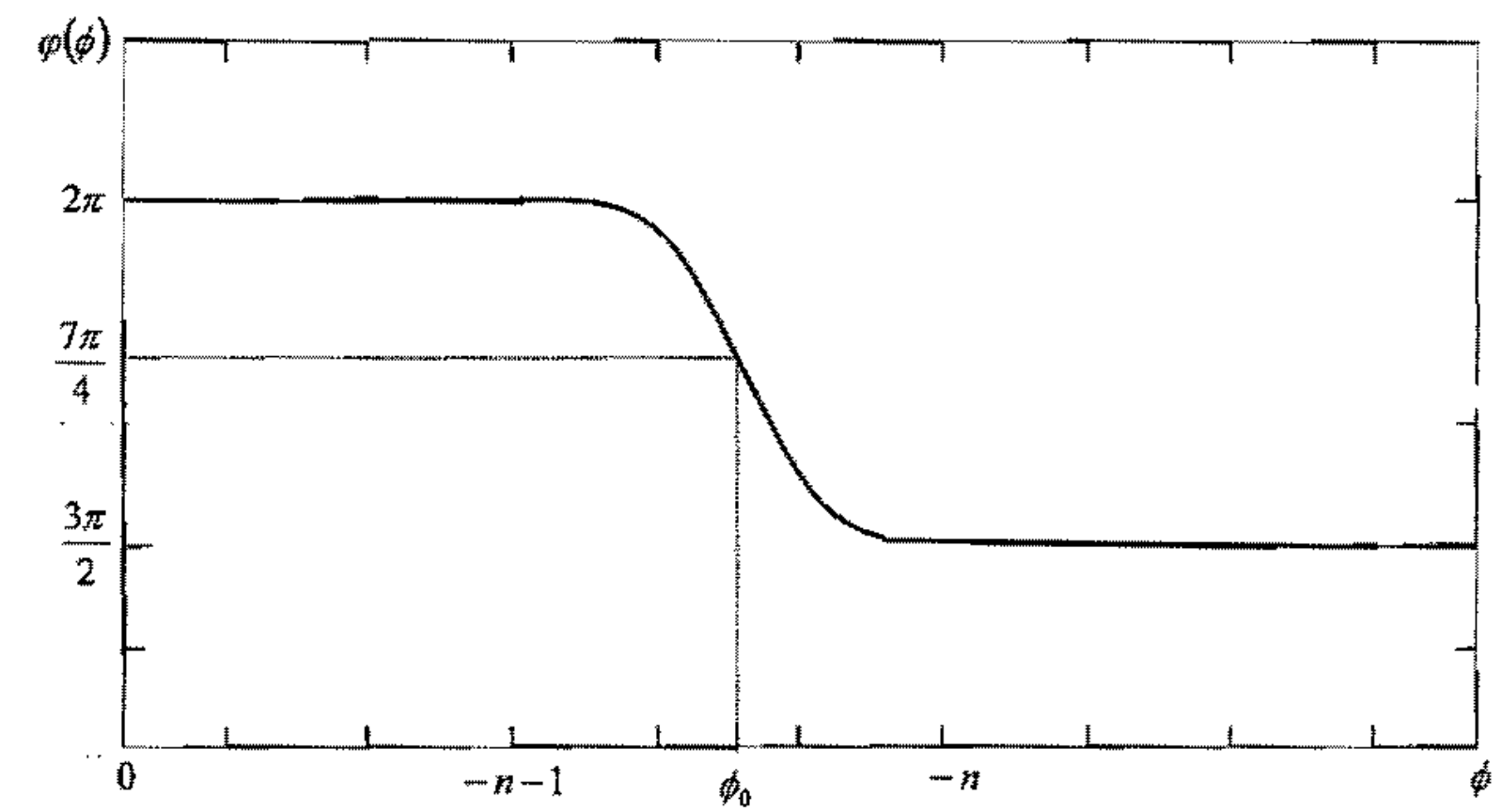

Figura $4 a$ - Interpolação com conservação da helicidade para o parâmetro $\varphi$ como função do fluxo magnético $\phi$ (para momento angular negativo n) quando n,é ímpar. 


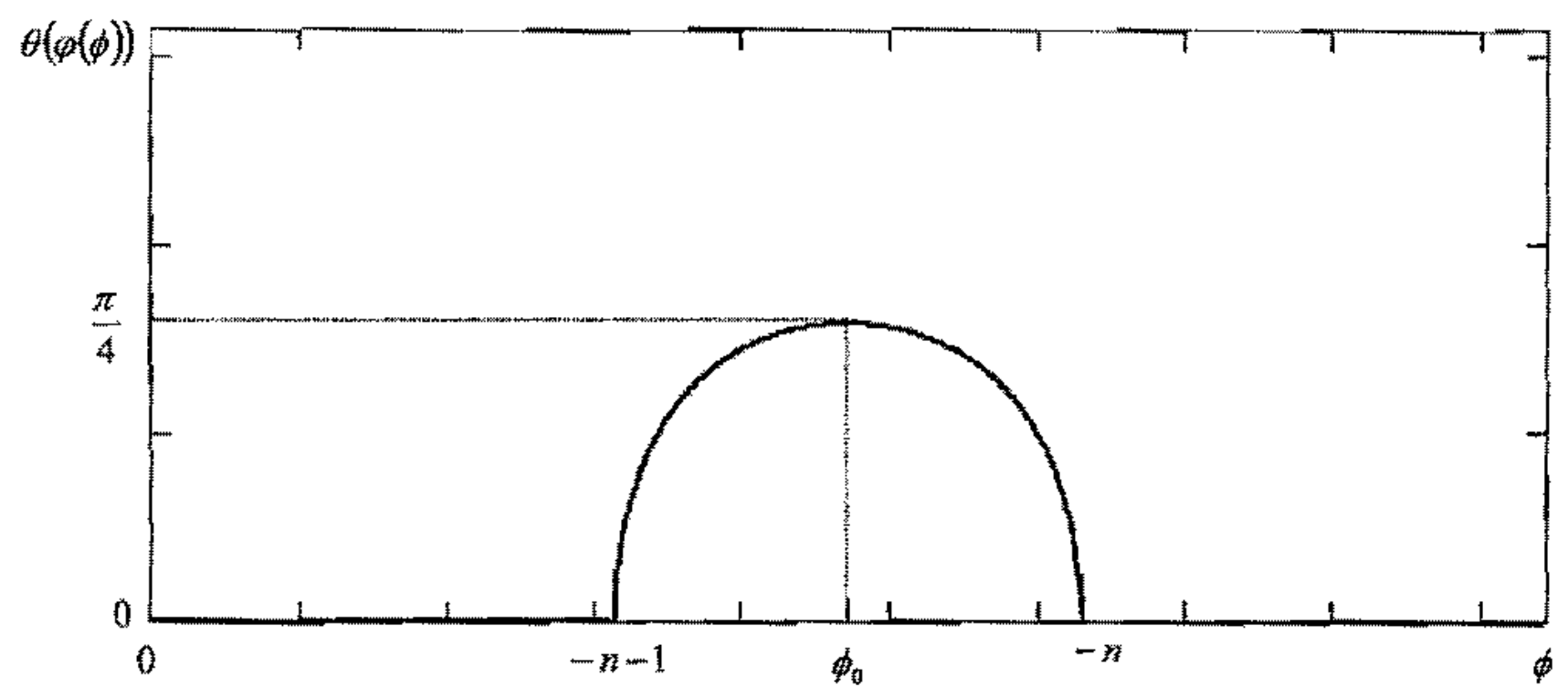

Figura $4 b$ - Interpolação com conservação da helicidade para o parâmetro $\theta$ como função do fluxo magnético $\phi$ (para momento angular negativo n) quando né ímpar. 


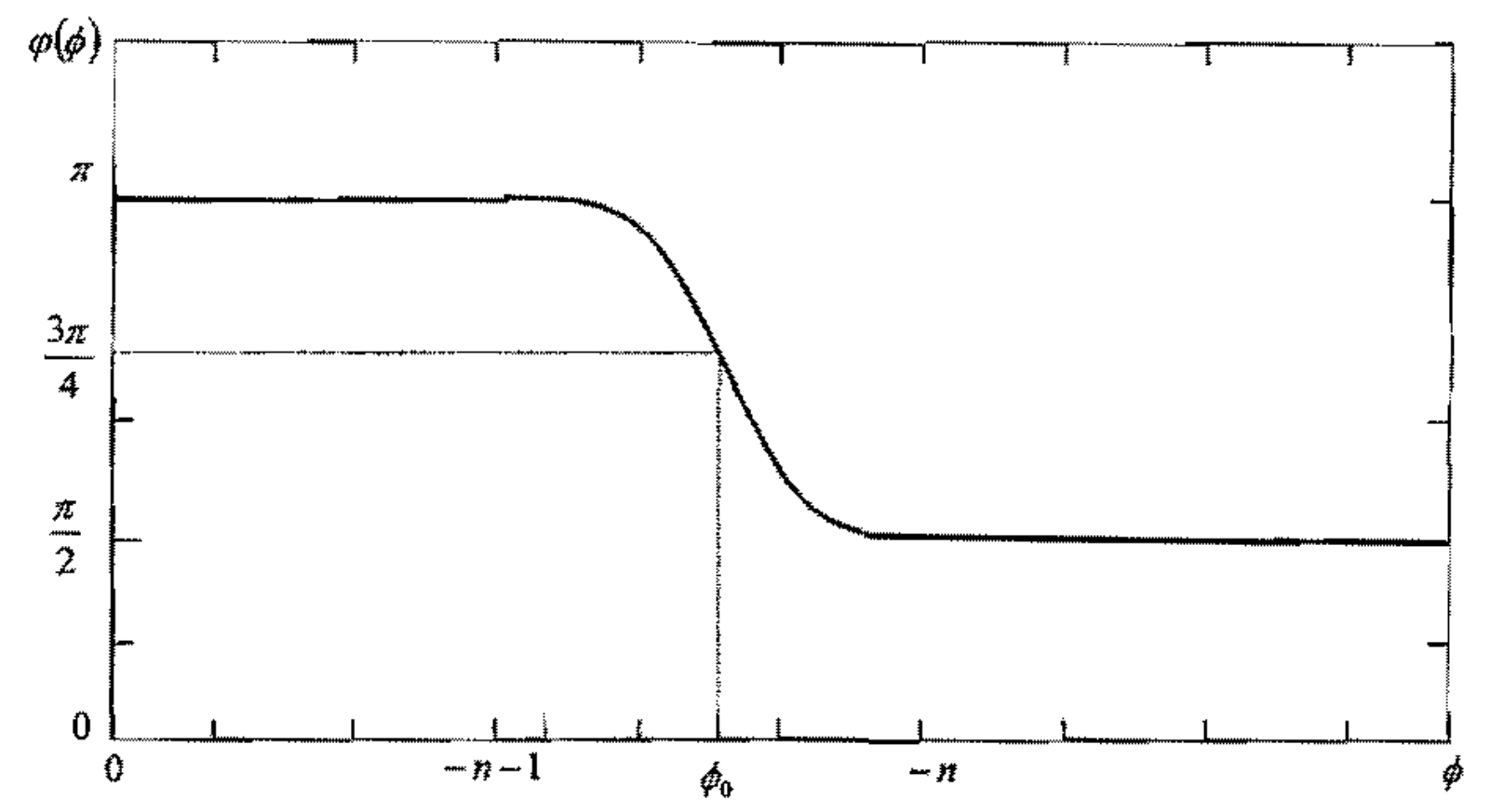

Figura $4 c$ - Interpolação com conservação da helicidade para o parâmetro $\varphi$ como função do fluxo magnético $\phi$ (para momento angular negativo $n$ ) quando $n_{3}$ á ímpar. 


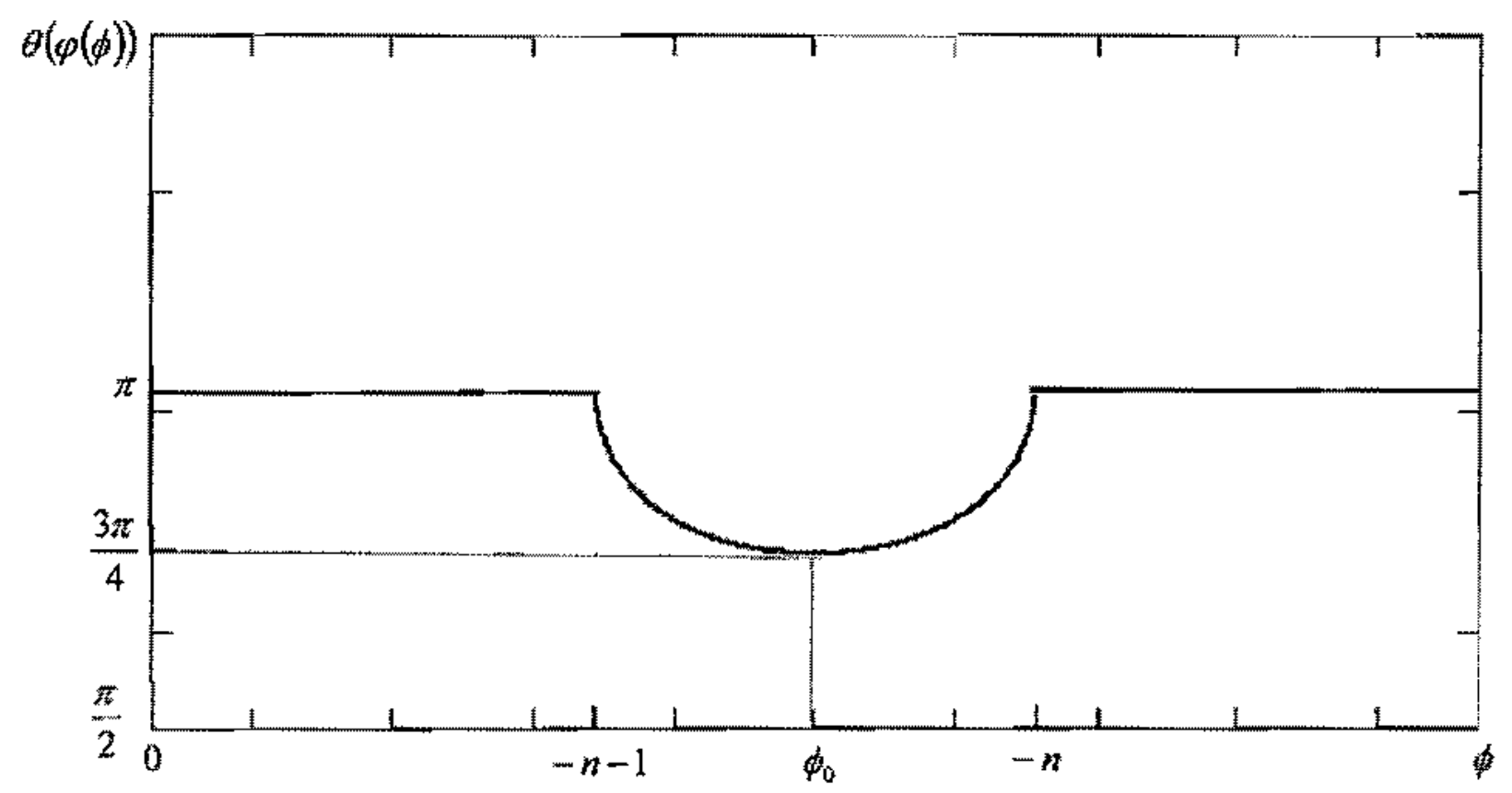

Figura 4d - Interpolação com conservação da helicidade para o parầmetro $\theta$ como função do fluxo magnético $\phi$ (para momento angular negativo n) quando né ímpar. 


\title{
Capítulo 10 \\ Determinaçāo das condiçōes de contorno a partir de um procedimento limite
}

\author{
10.1. Introduçäo - Procedimento Limite.
}

Nos capítulos anteriores resolvemos o problema de determinagăo dos dombinos mais gerais nos quais os operadores $I H$ e $\Lambda$ são auto-adjuntos e comutam (e consequentemente admitem auto-funçố comuns) e a simetria de Aharonov-Bohm ê preservada. Vamos agora, nesse capítulo, tratar de um outro procedimento utilizado nas referencias $[2],[4],[5],[6]$ e [7], que não consiate em se proceder a uma extensäo automadjunta para o operador $H$, mas que tantvém pode ser utilizado para a scolh das condicones de contorno e para a determinacão das auto-fungöes de $H$.

Vimos no Capitulo 2 que, para uma partícula de Dirac livre em duas dimensóes, o problema da determinaçăo das auto-funçós do hamiltoniana pode ser facilnente resolvido, una vez que a única condiçäo de contorno admissivel é a usual: funçóe de onda regulares na origem, caso contrário as auto-funçôes năo säo nomalizáveis na vizinhavęa da origem. Em outras palavras, o operador haruittoniana do caso livre essencinlmente auto-adjunto, nāo requerendo (nem permitindo) extensões auto-adjuntas. Também conseguimas obter, com relativa facilidade, as auto-funçöes simultäneas dos operadores hamiltoniana e helicidade para o caso da partícula de Dirac livre. $O$ mesmo não ocorre quando introduzimos um tubo de fluxo magnético na origem. Nesse caso, para $u=n+\phi \geq 0$ e para $\nu \leq-1$, novamente somente soluçoes regulares na origem săo admissiveis, no entanto, no intervalo aberto $-1<\nu<0$, é impossivel impor a condiçäo de regularidude na origem simultaneamente para todas as componentes das auto-

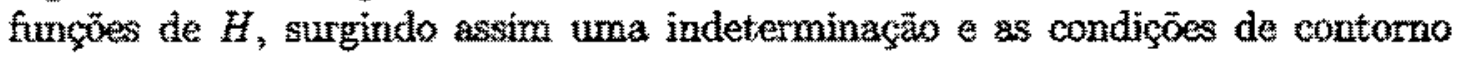
devern ser escolhidas dentro de uma familia de condiçoes de contorno axmissíveis. Foi demonstrado pela primeira vez por Sousa Gerbert ${ }^{(3)}$ e repetido ne trabalho no Capítulo 5, que existe uma familia a um parâmetro de extensōes unto-adjuntas possíveis para o operador $H$. As auto-funçóes parametrízadas pelo parâmetro $\theta$ encontradas por Sousa Gerbert ${ }^{[3]}$ tem o comportamento na origem: 


$$
\hat{\psi}(r) \sim\left[\begin{array}{cc}
i(m r)^{*} \operatorname{sen}\left[\frac{\pi}{4}+\frac{\theta}{2}\right] \\
(m r)^{-(1+\nu)} \cos \left[\frac{\pi}{4}+\frac{\theta}{2}\right]
\end{array}\right] .
$$

Surgindo assim naturalmente a questào de como resolver a ambiguidade, de qual dinâmika ê fisicamente correța.

Algumas tentativas aparecem na literatura no sentido de procurar responder assa perguntat

Alford et al ${ }^{[4 j}$ tentam uma motivaçäo fisica para a escolha das condiçoes de contorno dentre as possíveis, calculando as anto-funçōes do problema de uma particula de Dirac na presença de um tubo de fluxo magnético (com fluxo na superficie do thbo) de raio $R>0$, removendo, assim, a ambiguidade e tomando o limite $R \rightarrow 0$ no final dos cálculos. Esse mesmo procedimento é repetido por Hagen ${ }^{[5,5,7]}$ e Coutinho e Perez ${ }^{[2]}, 0$ conteúdo pertinente às referencias $[5]$, [6] e [7] está discutido separadamente no Apêndice D.

No entanto, embora não haja nada de errado com os cálculos das referências [2]: $[4],[5],[6]$ e $[7]$, as condicóes de contorno assim obtidas, conduzern a urna descontimuidade da dinâmica cono fungāo do fiuxo magnético $\phi$, produzindo também uma quelsra dis simetria de Aharonov-Bohm $(\phi \rightarrow \phi+1)^{[2,4]}$, apesar da conservacăo da helicidade. Assim, não é possivel a conservacão de ambas as simetrias (heljcidade e Aharonov-Bohm) para tal dinâmica. Na referência (2) é mostrado que é possivel, por interpolaçăo, obter a preservaçäo da simetria de Aharonov-Bohrn, nas, nesse caso, nâo é mais possivel a conservaçăo da helicidade.

Un outro procedimento limite apresentado na mesma referência [2] consiste em considerar o mesmo tubo de fluxo de raio $\bar{R}>0$, mas com a adição de un potencial repulsivo constante $u_{R}$ no sea interior. Tomandomse os limites $u_{R} \rightarrow \infty$ e $R \rightarrow 0$ de um certo modo particular conveniente, e possivel também obter todas as condigōes de contorno possíveis a um parâmetro ${ }^{[2]}$. 


\subsection{Modelo do Tubo de Fluxo.}

Nessa seçăo, vamos considerar o modelo do tubo de fluxo das referências [2], [4], $[3],[5],[6]$ e [7]. Nosso objetivo aqui é discutir principalmente os resultados das referências [2], [4] e compará-los com os resultados que obtivemos no Capitulo 5 para as auto-funções de $H$ e $\Lambda$.

Assim, vamos considerar o problema de uma particula de Dirac em duas dimensöes na presengra de um tubo de fluxo do tipo delta $\delta$ de casca, isto é, vamos tomar o potencial vetor $\vec{A}$, corno sendo:

$$
\vec{\nabla} \wedge \vec{A}=\vec{H}=\frac{2 \pi c}{e} \phi \frac{\delta(r)}{r} \vec{k} .
$$

Vamos introduzir também um potencial repulsivo $V_{R}(r)$, de tal modo que a equação radial (de duas componentes) de auto-funçôe da equação (2.2.14) se torna:

$$
H(r) \psi(r)=\left[\begin{array}{cc}
m s & -i\left(\partial_{r}+\frac{\psi(r)+1}{r}\right) \\
-i\left(\partial_{r}-\frac{\psi(r)}{r}\right) & -m s
\end{array}\right]=\left[E-V_{R}(r)\right] \psi(r)
$$

onde:

$$
\nu(r)=\left\{\begin{array}{cc}
n, & r<R \\
n+\phi, & r>R
\end{array}\right.
$$

e:

$$
V_{R}(r)=\left\{\begin{array}{cc}
u_{R}, \quad r<R \\
0, \quad r>R
\end{array} .\right.
$$

Vamos introduzir:

$$
\psi(r)=\left(\begin{array}{c}
\psi_{1}(r) \\
\psi_{2}(r)
\end{array}\right)
$$

Para $r \leq R$, a equaçäo radial para a componente $\psi_{1}$ é:

$$
\left\{\partial_{k_{r} r}^{2}+\frac{1}{k_{0} r} \partial_{k_{0} r}+\left(1-\frac{n^{2}}{\left(k_{0} r\right)^{2}}\right)\right\} \psi_{1}=0
$$


onde definimos:

$$
k_{0}=\sqrt{\left(E-a_{n}\right)^{2}-m^{2}}
$$

com a componente $\psi_{2}$ relacionada com a componente $\psi_{1}$ através da equaça:

$$
\psi_{2}=-\frac{k_{0}}{E-u_{R}+m s}\left(\partial_{k^{*}}-\frac{n}{k_{0 \%}}\right) \psi_{1}
$$

Usando a equasăo $(2.2 .27)$ e lembrando que ${ }^{[10)_{*}}$

$$
y_{n}(x)=(-)^{n} y_{n}(x)
$$

devemos ter:

$$
\psi\left(k_{0} r\right)=\frac{1}{N}\left[\begin{array}{c}
J_{n}\left(k_{0} r\right) \\
\frac{i_{k_{0}}}{E-u_{n}+m s} J_{n+1}\left(k_{0} r\right)
\end{array}\right], \quad r<R
$$

onde $N$ é urn fator de normalizaçä́ e $n$ pode ser positivo ou negativo.

Para $\geq R$, a equaçăo radial para 靬

$$
\left\{\partial_{k r}^{2}+\frac{1}{k_{r}} \partial_{k r}+\left(1-\frac{v^{2}}{(k r)^{2}}\right)\right\} \psi_{1}=0
$$

onde:

$$
\hbar=\sqrt{E^{2}-m^{2}}
$$

e com a componente $\psi_{2}$ relacionada com a componente $\hat{\psi}_{1}$ através da equaçä

$$
\psi_{2}=\frac{-i k}{E+m s}\left(\partial_{k r}-\frac{\nu}{k r}\right) h_{1}
$$

Nesse caso, as possiveis aluçoes dependem de em que intervalo está $\nu$ (para $y>0$ e $z<-1$ as soluçoes säo as soluçôs regulares). Lembrando que estamos tratando do caso $-1<y<0$, as soluçōes mais gerais são da forma:

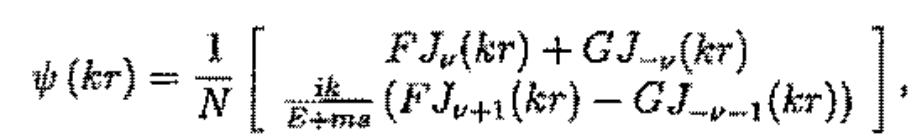

onde $N$ é um fator de normalizagāo. 
As constantes $F$ e $G$, que regularm respectivamente as proporçoes das fungōes singular (regular) e regular (singuar) da componente $\psi_{1}\left(\psi_{2}\right)$ podem ser encontradas, impondo a contimuidade em $r=R$ para ambas as componentes, isto é:

$$
\begin{gathered}
y_{n}\left(k_{0} R\right)=F J_{v}(k R)+G_{n}(k R) \\
\frac{i k_{0}}{E-u_{R}+m s} J_{n+1}\left(k_{0} R\right)=\frac{i k}{E+m s}\left[F J_{v}(k R)-G J_{m,-1}(k R)\right]
\end{gathered}
$$

Esse sistema de equagoes pode ser resolvido formecendo:

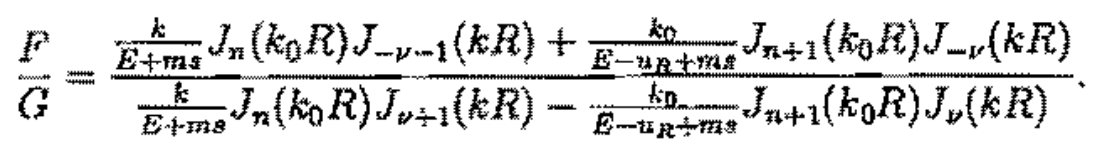

Lembrando do comportanento na origem das funçoes de Bessed ${ }^{[10]}$ dado pela equação (5.5.6) e tomando o limite $R \rightarrow 0, \mathrm{com} V_{R}$ mantido constante na expressäo da equaçăo $(10.2 .18)$ para $n \geq 0$ e $n \leq 0$, obtemos:

$$
\frac{E}{G} \ln _{n \rightarrow 0} \propto R^{-2(t+1)} \quad \text { para } n \geq 0
$$

e

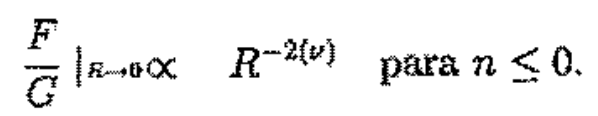

Note que esses resultados são os mesmos encontrudos por Alford ${ }^{[4]}$, se lembrarmonos que $\nu=n+4$.

Lembremo-nos também do seguinte fato: para todo valor nảo inteiro do parämetro do fluxo 6 há axatamente um valor de $n$, ou seja, uma onda parcial, $n=-[\phi]-1$ ([申] é o maior inteiro menor que $\phi$ ) para a qual teremos o problema da indeterminaça do domínio do hamiltoniana, isto é, $v$ est no intervalo crítico ]-1, $0[$.

Assim para $n>0$, que corresponde a $\hat{\varphi}<0$, a equaça (10.2.19) fornexe soluçẫo singular para $\psi_{1}$ e consequentemente, devido a equagāo (10.2.14), solução regular para $\psi_{2}$, o que corresponde a fazer a escolha do parâmetro $\theta$ da referência [3] como sendo $\frac{\pi}{2}$ na equação $(10.1 .1)$ a $\delta=\frac{\pi}{2}$ nas equaçós $(5.4 .2)$ e $(5.4 .3)$.

Para $n<0$, que corresponde $a \phi>0$, a equaça $(10.2 .20)$ fornece soluça singular para Wi e consequentemente, devido a equaçào $(10,2.14)$, soluģa regular 
para $\psi_{2}$, o que corresponde a fazer a escolha do parâmetro $\theta$ da referêncta [a] como sendo $-\frac{\pi}{2}$ na equaçäo (10.1.1) ou $\delta=0$ na equaçöes $(5.4 .2)$ e $(5.4 .3)$.

Assim, através desse procedimento limite obternos as condiçöes de contorno das referências [2], [4], isto é, o parâraetro $\theta$ da extensäo utilizado ne referência [3] feca sendo:

$$
\theta=-\operatorname{sgn}(\phi) \frac{\pi}{2}
$$

As soluçōes assim obtidas para as auto-funções (de duas componentes) da equaf̧

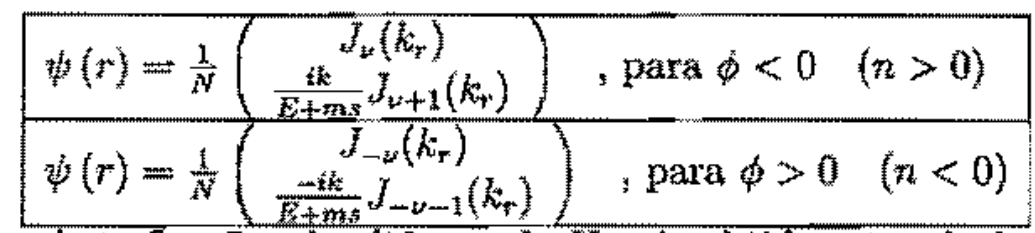

Tabela 12 - Auto-funçồ simultâneas de $H$ e $\Lambda_{\text {, }}$ obtidas através da solução do problema com um tubo de fluxo magnético de raio $R>0$ e depois tomando-se o limite $P \rightarrow 0$ no final dos calculos $[2,14-7$.

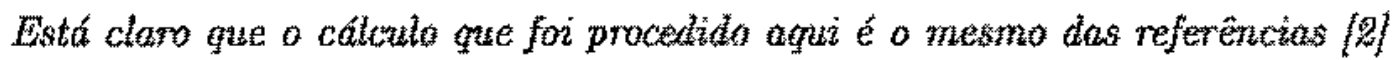
e [4]. No cratanto, taluez näo esteja claro que é mesmo cálculo feito por Haw

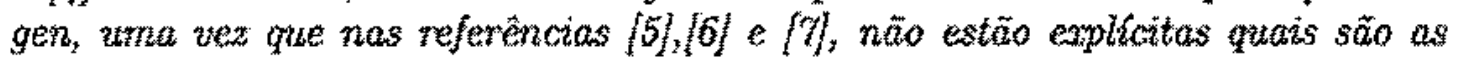
condições de contomo que as funçöes de onda devem obedecer. Os resultados das raferéncias [5],[6] e [7] estão discutidos separadamente no Apêndice D.

As auto-finçōes de $H$ apresentajas na Tabela 12, obtidas por esse processo limite, também são auto-funçōes do operador $A$, como pode ser verificado por mera aplicaga de de $\Lambda$ nessas funçoes, tomando-se as constantes $\alpha$ e $\beta$ como na Tabela 4. On de outra forma, podemos notar que as soluçöes da Tabela 12 são

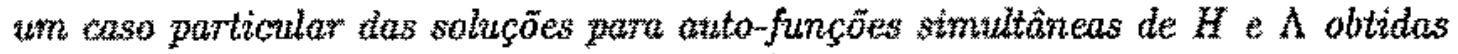
no Capitulo 5 (etuaçổ (5.9.5) a (5.9.12)).

Apesar da conservacäo da helicidade, a dinânica dada pela condiçâo de contorno da equagão (10.2.2.) nמื้ preserva outra importante simetria: a sinetria de Aharonov-Bohm, produzindo também uma descontimuidade na dinämica (isto é, das condigâs de contorno e das auto-funçōes ) como fungäo do parâmetro $\theta$ para $\phi=-n$, se $n<0$ e para $\phi=-n-1$, se $n \geq 0$. Vejamos como isso acontece.

Para $\nu \geq 0$, dissemos que a única condicão de contorno admissivel é a regularidade na origen, o que corresponde ao parâmetro da extensäo da referência [3] igual a $\frac{\pi}{2}$ (veja equação (10.1.1)). Para $\nu \leq-1$, novamente a única condiçăo de 
contorno admissivel é a regularidade na origern, o que pode ser obtido tomando-se $\theta=-\frac{\pi}{2}$ na equação (10.1.1). Assim, para um dado valor do momento angular $n$ temos:

$$
\begin{gathered}
\theta(\phi)=\frac{\pi}{2} \quad \text { para } \phi \geq-n \\
\theta(\phi)=-\frac{\pi}{2} \quad \text { para } \phi \geq-n-1
\end{gathered}
$$

Na regiầ crítica, para a qual $-n-1<\phi<-n$, (isto é $-1<\nu<0$ ) temos:

$$
\theta=-\operatorname{sgn}(\phi) \frac{\pi}{2}
$$

Vamos considerar primeiro o caso $n<0$, para o qual a regita crítica $(-1<$ i $<0$ ) ocorre para $4>0$. 0 comportamento de $\theta$ como funça de $\phi$, nesse caso é ilustrado no gráfico abaixo, para um dado valor de $n$ negativo:

Figuma 5.

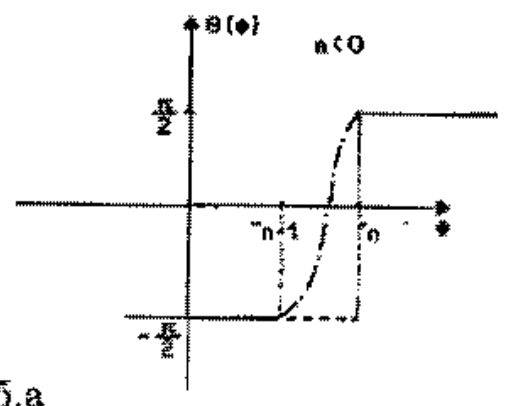

Vemos que a deseontinuidade na dinänica ocorre para $6=-n$.

Para caso $n>0$, a regiäo crítica ocorre para $<0$. O gráfico da figura 5 b abaixo ilustra o comportamento do parâmetro $\theta$ da extensäo da referência [3] como finção do fluxo $\$$ para urn dado valor de $n>0$.

Figura $5 b$

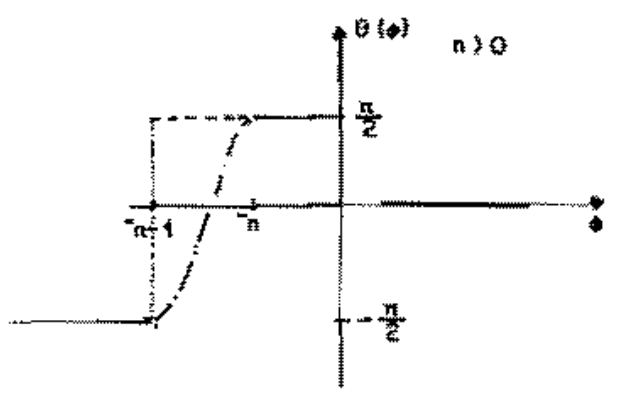

Vemos que a descontinuidade no dinâmica ocorre para $\phi=-n-1$. 
A. assimetria entre os gráficos das figuras 5.a para $n<0$ e 5.b para $n>0$ e a descontinuidade da dinânica produzida pela equaçāo (10.2.24) para todo valor inteiro do fluxo $\phi$ (para $\phi=-n$ se $n<0$ e para $\phi=-n-1$ se $n>0$ ) explica a quebra da simetria $\phi \rightarrow \phi+1$ do hamiltoniana (basta observar o que ocorre quando o fluxo $\phi$ vai em $\phi+k$ trocando de sinal, devido a assimetria da condicão de contomo da exuação (10.2.24)).

Note que a descontinuidade nunca ocorre para $\phi=0$, como erronearnente foi afirmado na referência [4].

Se quisermos obter a continuidade da dinâmica, preservando a simetria $\phi \rightarrow$ $\phi+1$, podemos pensar para um dado valor de $n$ (positivo ou negativo) em interpolar a função:

$$
\begin{gathered}
\theta(\phi)=\frac{\pi}{2}, \phi \geq-n \\
\theta(\phi)=\frac{-\pi}{2}, \phi \leq-n-1,
\end{gathered}
$$

no intervalo aberto $-n-1<\phi<-n$. Assim, vamos tomar:

$$
\theta(\phi)=f(\phi+n+1), n-1<\phi<-n,
$$

onde $f$ é una função cuja forma é independente de $n$ e contínua no intervalo $[0,1]$. Impondo que $f(0)=-\frac{\pi}{2}$ e $f(1)=\frac{\pi}{2}$, garantimos a continuidade. Veja os gréficos das figuras $5 a$ e $5 b$, onde as curvas pontilhadas correspondem as interpolacóces para as casos de $n>0$ (figura $5 \mathrm{a}$ ) e $n>0$ (figura $5 \mathrm{~b}$ ). No entanto, procedendo dessa forna, isto é, tomando o parấmetro $\theta$ no intervalo crítico corno sendo dado pela equação acima, embora garantamos a preservaçäo da simetria $\phi \rightarrow \phi+1$ e a continuidade da dinămica como funçäo de $\phi$, perderemos a possibilidade de conservação da helicidade, uma vez que as soluçóes da forma da equaça $(10.1,1)$ da mesran forma que as soluçōes a um parâmetro (Tabela 2) do Capítulo 5 nầ conservam a helicidade, como foi mostradio na Secäo 5.8 .

De modo que não é possivel, nessa situação, a preservaçäo de ambas as simetrias (helicidade e Ahronov-Bohm) simultanearnente. Isso näo significa que para extensões auto-adjuntas de $H$ mais gerais que a da referência [3] não possam haver dinâmicas que conservem ambes as simetrias. De fato, conforme verificamos no Capítulo 9 anterior, as extensões que encontrarmos nos capitulos 7 e 8 além de preservarem a helicidade podem também preservar a simetria $\phi \rightarrow \phi+1$ para certas escolhas especiais dos intervalos de variaçâo dos parâmetros das extensōes. 


\subsection{Processo limite para a obtençäo de todas as extensöes a um parâmetro.}

Vamos agora mostrar que, para o caso critico de $-1<v<0$, tomando os limites $w_{R} \rightarrow \infty$ e $R \rightarrow 0$ em certas proporçoes, podemos obter todas as condiçós de contorno a urn parämetroli!

Vamos tomar o limite $u_{R} \rightarrow \infty$ e $R \rightarrow 0$ na expressão da equaçăo (10.2.18), da seguinte forrna:

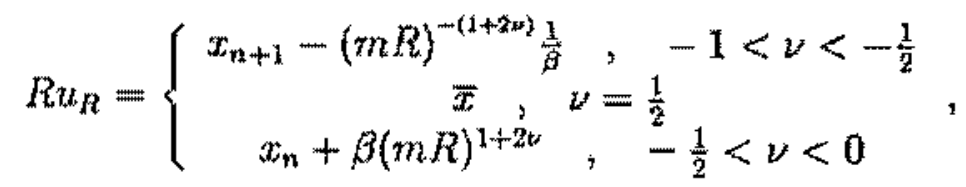

onde $\widetilde{x}_{n} \neq 0$ é o primeiro zero de $J_{n}(x)$, $\dddot{x}$ é qualquer número tal que $J_{n}(x) \neq 0$ e $\beta$ e uma constante adimensional. Lembrando do comportamento na origern das funçoes de Bessel (equasäo (5.5.6)) a equaçäo (10.2.18) pode ser escrita como:

$$
\left.\frac{F}{G}\right|_{n \rightarrow \infty+\infty}=\frac{\sqrt{\frac{E-m s}{E+m s}} \frac{1}{\Gamma(-\nu)}\left(\frac{k R}{2}\right)^{-i-v} J_{n}\left(u_{R} R\right)+\frac{1}{\Gamma(1-\nu)}\left(\frac{k R}{2}\right)^{1-\nu} J_{n+1}\left(u_{R} R\right)}{\sqrt{\frac{E-m s}{E+m s}} \frac{1}{\Gamma(2+\nu)}\left(\frac{k R}{2}\right)^{1+\nu} J_{n}\left(u_{R} R\right)-\frac{1}{\Gamma(1+\nu)}\left(\frac{k R}{2}\right)^{\nu} J_{n+1}\left(u_{R} R\right)}
$$

Considerando primeiro o caso $-1<\nu<-\frac{1}{2}$, temos:

$$
J_{n}\left(w_{R} R\right)=J_{n}\left(x_{n+1}-\frac{1}{\beta}(m R)^{-(1+2 k)}\right) \sim J_{n}\left(x_{n+1}\right)
$$

e

$$
J_{n+1+1}\left(x_{n} R\right)=J_{n+1}\left(x_{n+1}-\frac{1}{\beta}(m R)^{-(1+2 n)}\right) \sim J_{z+1}\left(x_{n+1}\right)-\frac{1}{\beta}(m R)^{-\{1+2 n)} J_{n}^{z}\left(x_{n+1}\right)
$$

Usando o resultado da equação (5.5.6), a equação (10.3.4) acima fornece

$$
J_{n+1}^{\prime}\left(x_{n+1}\right)=J_{n}\left(x_{n+1}\right)-\frac{n+1}{x_{n+1}} J_{n+1}\left(x_{n+1}\right)
$$

e, portanto:

$$
J_{n+1}\left(R u_{R}\right) \sim-\frac{1}{\beta}(m R)^{-(1+2 x)} J_{n}\left(z_{n+1}\right)
$$


Substituindo os resultados das equagotos (10.3.3) e(10.3.6) na equar obtemos:

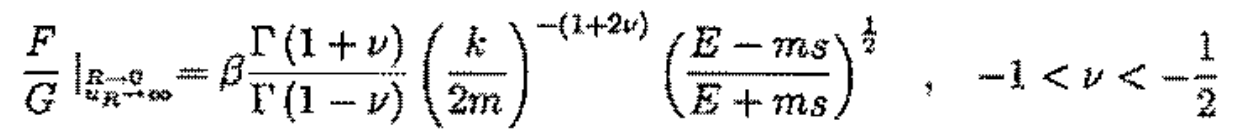

Considerando agora o caso $-\frac{1}{2}<y<0$, temos:

$$
J_{n+1}\left(R u_{n}\right)=J_{n+1}\left(x_{n}+\beta(m R)^{1+2 n}\right) \sim J_{n+1}\left(n_{n}\right)
$$

e

$$
J_{m}\left(R u_{n}\right)=J_{n}\left(x_{n}+\beta(m R)^{2+2 n}\right) \sim J_{n}\left(x_{n}\right)+\beta(m R)^{1+2 k} y_{n}^{\prime}\left(x_{n}\right)
$$

Usando novamente o resultado da equaçäo $(5.5 .6)$ na equacäo $(10.3 .9)$ acima, temos:

$$
J_{n}^{\prime}\left(x_{n}\right)=-J_{n+1}\left(x_{n}\right)+\frac{n}{x_{n}} J_{n}\left(x_{n}\right)
$$

e, portanto:

$$
J_{n}\left(R u_{R}\right) \sim-\rho(m R)^{1+t_{2}} J_{n+1}\left(w_{n}\right)
$$

Substituindo, os resultados das equações (10.3.8) e (10.3.11) na equaçäo (10.3.2), obtemos:

$$
\left.\frac{F}{G}\right|_{m_{n \rightarrow \infty} \rightarrow \infty}=\frac{\Gamma(1+\nu)}{\Gamma(-\nu)}\left(\frac{k}{2 m}\right)^{-(1+2 v)}\left(\frac{E-m_{s}}{E+m_{s}}\right)^{\frac{1}{2}}, \frac{-1}{2}<\nu<0
$$

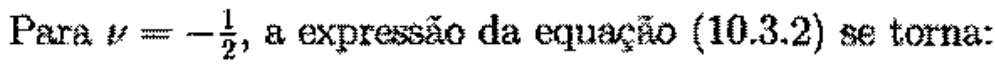

$$
\left.\frac{F}{G}\right|_{g_{n-0}-0}=-\frac{J_{n}(x)}{J_{n+1}(x)}\left(\frac{E-m s}{E+m s}\right)^{\frac{1}{2}} \text {. }
$$


Assim, tomando os limites $u_{R} \rightarrow \infty$ e $R \rightarrow 0$ como na equacäo (10.3.1), obtemos:

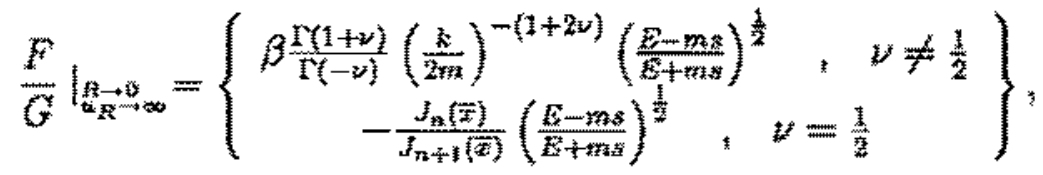

o que ê $\sigma$ mesmo que tomar:

$$
2^{-\nu-\frac{1}{2}} \frac{\Gamma(-\nu)}{\Gamma(\nu+1)} \operatorname{tg} \delta=\operatorname{tg}\left[\frac{\theta}{2}+\frac{\pi}{4}\right]=\left\{\begin{array}{cc}
\beta, & y \neq \frac{1}{2} \\
-\frac{J_{n}(2)}{J_{n+1(1)}(x)}, & y=\frac{1}{2}
\end{array}\right\}
$$

na exuaçăo (10.1.1), onde $\theta$ é o paränetro da referência [3] e $\delta$ é o parâmetro da extensão da referência [1] e também das equaçôs (5.4.1) e (5.4.2) dessa tese. Dessa forma, obtemos, portanto, todas as condiçöes de contorno admissiveis a urn parämetro (parâmetro $\beta$ ). Provavelmente, existe também alguma física expressa através de algum processo limite como este da referência [2], que acabamos de reproduzir, que leve às condiçöes de contorno obtidas nos Capítulos 7 e 8, as quais além de conservarem a helicidade, preservam também a simetria $\phi \rightarrow \phi+1$. No entanto, certamente näo é obvio qual dove ser sse processo limite. 


\section{Capítulo 11 Conclusōes}

É importante salientar aqu alguns aspectos interessantes sobre as condiçỏes de contorno que obtivernos nesse trabalho.

Em primeiro lugar observernos que as condiçoes de contomo das equaçós (8.1.8) e (8.1.9) caracterizam os domínios mais gerais onde o operador hamithniana é auto-adjunto e incluem todas as condicooes de contorno apresentadas nas referências [1], [2], [3], [4], [5], [6] e [7].

Apesar de os operadores $H$ e $\Lambda$ formalmente comutarem, simplesmente a definişâa dos dous operadores num mesmo domínio denso comum, nâo garante a comutaçäo efetziva entre esses operadores a consequente obtenção de autofunçöes comans, contrarianente ao que se afirmou na referência [1].

Mostramos que é formalmente possivel obter auto-fincỡes comuns a $H$ e $\Lambda$ para certas combinacōes particulares das auto-funcôes de $H$ (equação $(2.2 .17)$ ) dadas na Tabela 2 com a condição da equação da equação (5.8.13) satisfeita.

Irnpondo às auto-funçöes de $H$ que satisfazern às condiçoes de contorno mais gerais das equaçōes (8.1.8) e (8.1.9) que tanbém satisfaçam à condiçầo (5.8.13) de conservaçấo da helicidade, obtivemos as relaçōes entre os parâmetros da extensão apresentadas na Tabela 8. Dessa forma encontramos os dornínios mais gerais onde a helicidade é conservada.

Alëm da conservaçâa da helicidade, concluirnos que uma característica importante das condiçöes de contorno que obtivemos é que elas permitern tambén a preservaçäo da simetria de Aharonov-Bohm. Para certas escolhas convenientes dos intervalos de variação dos parâmetras das extensỏes, é passível, atravếs de uma interpolaçãa, obter a preservaçâo da simetria de Aharonov-Bohm $(\phi \rightarrow \phi+1)$.

De modo que, a conservaçầ da helicidade se toma compatźzel com a simetria de Aharunov-Bohn para as condiçöes de contomo que obtivemos, o que näo ocorre para as demais condiçöes de contomo obtidas na literatura! ${ }^{[-7]}$.

No entanto, a conservaçấo da helicidade com a preservação da simetria de Aharonon-Bohm nâo ocorre apenas parn uma, mas para várias diferentes dinâmicas. 
De modo que, podemos tizer que nem a conservação da helicidade nem a simetria de Aharonou-Bohm podem remover a indeterminaçäo existente na escolha das condiçốes de contomo pora o caso de uma partícula de Diruc, que se move num plano na presença de uma linha de faxo magnético na origem.

A continuidade da dinâmica (isto é, das condiçôes de contorno a das funçốs de onda) como funçấo do fuxco magnético ф também é garantida, nesse caso. 


\section{Apêndice A \\ Cálculo da Relação de Comutação $[H, \Lambda]$}

A relação de comutação formal entre os operadores $H, \Lambda$ :

$$
[H, \Lambda]=0,
$$

pode ser facilmente verificada, usando-se as definiçōes formais desses operadores dadas respectivamente pelas equações (2.1.1) e (2.1.13). Basta notar que $H$ (dado pela equação (2.1.1)) é o operador diagonal:

$$
H=\left(\begin{array}{cc}
\vec{\pi} \cdot \vec{\sigma}+\sigma_{3} m & 0 \\
0 & \vec{\pi} \cdot \vec{\sigma}-\sigma_{3} m
\end{array}\right)
$$

e $\Lambda$ dado pela equação (2.1.13) é um operador não diagonal;

$$
\Lambda=\left(\begin{array}{cc}
0 & \vec{\pi} \cdot \vec{\sigma} \\
\vec{\pi} \cdot \vec{\sigma} & 0
\end{array}\right)
$$

No entanto, para sermos mais cuidadosos, devemos checar se a açāo desses operadores numa função de onda genérica é de fato comutativa como parece. Para isso vamos tomar os operadores $H$ e $\Lambda$ como sendo dados pelas equaçōes (2.2.7) e (2.3.2), depois de feita a transformação de variáveis para coordenadas polares.

Assim, sejam os operadores $H$ e $\Lambda$ (escritos em coordenadas polares).

$$
H=\left(\begin{array}{cc}
\mathcal{H}+\sigma_{3} m & 0 \\
0 & \mathcal{H}-\sigma_{3} m
\end{array}\right)
$$

$\mathrm{e}$

$$
\Lambda=\left(\begin{array}{cc}
0 & \mathcal{H} \\
\mathcal{H} & 0
\end{array}\right)
$$

onde $\mathcal{H}$ é dado pela equação (2.2.2) aqui transcrita:

$$
\mathcal{H}=\left(\begin{array}{cc}
0 & -i e^{-i \theta}\left(\partial_{r}-\frac{i}{r} \partial_{\theta}+\frac{\phi}{r}\right) \\
-i e^{i \theta}\left(\partial_{r}+\frac{i}{r} \partial_{\theta}-\frac{\phi}{r}\right) & 0
\end{array}\right)
$$


Calculando inicialmente a açäo do operador $H$ A sobre uma funçäo de onda genéricha de quatro componentes:

$$
\psi=\left(\begin{array}{c}
\psi_{1} \\
\psi_{2}
\end{array}\right)
$$

onde $\psi_{1}, \psi_{2}$ sāo espinores de duas componentes, temos:

$$
\begin{gathered}
H \Lambda \psi=\left(\begin{array}{cc}
H+\sigma_{3} m & 0 \\
0 & H-\sigma_{3} m
\end{array}\right)\left(\begin{array}{cc}
0 & \mathcal{H} \\
\mathcal{H} & 0
\end{array}\right)\left(\begin{array}{l}
\psi_{1} \\
\psi_{2}
\end{array}\right) \\
H \Lambda \psi=\left(\begin{array}{c}
\left.\mathcal{H}+\sigma_{3} m\right) H \psi_{2} \\
\left(\mathcal{H}-\sigma_{3} m\right) H \psi_{1}
\end{array}\right)
\end{gathered}
$$

Calculando a aça do operador $A H$ sobre a funcão de onda genérica de equacăo (A-5), temos:

$$
\begin{gathered}
\Lambda H \psi=\left(\begin{array}{cc}
0 & \mathcal{H} \\
\mathcal{H} & 0
\end{array}\right)\left(\begin{array}{cc}
H+\sigma_{3} m & 0 \\
0 & \mathcal{H}-\sigma_{3} m
\end{array}\right)\left(\begin{array}{l}
\psi_{1} \\
\psi_{2}
\end{array}\right) \\
\Lambda H \psi=\left(\begin{array}{c}
\mathcal{H}\left(H-\sigma_{3} m\right) \psi_{2} \\
H\left(H-\sigma_{3} m\right) \psi_{1}
\end{array}\right)
\end{gathered}
$$

É fál verificar, considerando que $M$ é o operador näo dìzgonal dado por (A-4), a seguinte equaçä:

$$
\mathcal{H} \sigma_{3} \psi_{i}=-\sigma_{3} \mathcal{H} \psi_{i}
$$

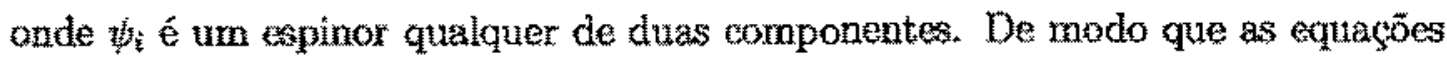
(A-6) e (A-7) produzem o mesmo resultado, implicando, automaticamente na

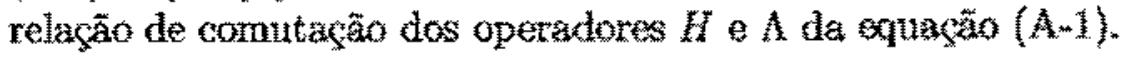




\section{Apêndice $\mathbf{B}$}

Cálculo das Relaçōes de Comutação $\left[H, S_{3}\right],\left[H, l_{z}\right],\left[H, y_{z}\right]$.

Consideremos o operador bamiltoniana formalmente definido pela equaca 30 (2.1.1) corno sendo:

$$
H=\vec{\alpha} \cdot\left[\vec{p}+\frac{e}{c} \vec{A}\right]+\beta m
$$

com o potencial vetor $\vec{A}$ dado pela equagän $(2.1 .11)$, e o operador de spin $S_{3}$ definido por:

$$
S_{3}=\frac{\sum_{3}}{2}
$$

Usando as equaçōes (B-1) e (B-2) acima a a equą̧ăo (2.1.11), podemos cscrever o comutador $\left[H, S_{3}\right]$ como sendo:

$$
\begin{gathered}
{\left[H, S_{3}\right]=\frac{1}{2}\left[\alpha_{1} p_{x}+\alpha_{1} p_{y}+\frac{e \phi}{c r^{2}}\left(-y \alpha_{1}+x \alpha_{2}\right)+\beta m, \Sigma_{3}\right]} \\
{\left[H . S_{3}\right]=\frac{1}{2}\left\{\left[\alpha_{1}, \Sigma_{3}\right] p_{x}+\left[\alpha_{2}, \Sigma_{3} \mid p_{y}+\frac{e}{6} \frac{\phi^{2}}{r^{2}}\left(-\left[\alpha_{1}, \Sigma_{3}\right] y+\left[\alpha_{2,}, \Sigma_{3}\right] x\right)+\left[\beta, \Sigma_{3}\right] m\right\}\right.}
\end{gathered}
$$

Através das definiçóes das matrizes $\alpha_{1}, \alpha_{2}, \theta$ e $\Sigma_{3}$ dadas respectivamente pelas equaçöe $(2.1 .4),(2.1 .5),(2.1 .6)$ e $(2.1 .21)$ e lembrando da relaçăo de comutação conhecida entre as matrizes de Fauli:

$$
\left[\sigma_{i}, \sigma_{j}\right]=2 i \varepsilon_{i j k} \sigma_{k}
$$

podernos escrever:

$$
\begin{gathered}
{\left[\alpha_{1}, \Sigma_{3}\right]=-2 i \alpha_{2}} \\
{\left[\alpha_{2}, \Sigma_{3}\right]=2 i \alpha_{1}}
\end{gathered}
$$




$$
\left[\beta, \Sigma_{3}\right]=0 .
$$

Substituindo os restltados das equaços (B-5) a (B-7) acìma na equação (B-3) obtemos:

$$
\left[H, S_{3}\right]=\left\{\left\{\alpha_{1} p_{y}-\alpha_{2} p_{x}+\frac{e \phi}{c}\left(\alpha^{2} \alpha_{1} x+\alpha_{2} y\right)\right\}\right.
$$

ou seja:

$$
\left[H, S_{3}\right]=\hat{i}\left\{\vec{\alpha} \wedge \vec{p}+\frac{e \phi}{e r^{2}}(\vec{\alpha}, \vec{T})\right\}
$$

Considerando, agora, o operador $l_{\alpha}$ dado por:

$$
l_{z}=x p_{y}-y p_{x z}
$$

podemos escrever o comutador $\left[H, l_{z}\right]$ como sendo dado por:

$$
\left[H, l_{z}\right]=\left[\alpha_{1} p_{x}+\alpha_{2} p_{y}+\frac{e}{\varepsilon \tau^{2}}\left(-y \alpha_{1}+x \alpha_{2}\right)+\beta m, x p_{y}-y p_{x}\right]
$$

Lembrando das relaçōes de comutaçäo conhecidas:

$$
[x, y]=\left[y, p_{x}\right]=\left[x_{1}, p_{x}\right]=\left[y, p_{z}\right]=0
$$

e:

$$
\left[x_{;}, z_{y}\right]=\left[y, p_{x}\right]=i
$$

a equação (B-10) pode ser escrita como sendo:

$$
\left[H, L_{z}\right]=\alpha_{1}\left[p_{x}, x\right] p_{y}-\alpha_{2}\left[p_{y}, y\right] p_{x}+\frac{e \phi}{c r^{2}}\left(-x\left[y, p_{y}\right] \alpha_{1}-y\left[x, p_{z}\right] \alpha_{2}\right)
$$

de onde vern que:

$$
\left[H, l_{z}\right]=-i\left(\vec{\alpha} \wedge \vec{p}+\frac{E}{e} \frac{\phi}{r^{2}} \vec{\alpha} \cdot \vec{r}\right)
$$


De modo que sendo o momento angular total:

$$
J_{z}=S_{3}+l_{*},
$$

e usando as equaçöes (B-8) e (B-13), obtemos imediatamente:

$$
\left[H_{3} J_{2}\right]=0 .
$$




\section{Apêndice $\mathrm{C}$}

Cálculo das Relaçöes de Comutação $\left[\Lambda, S_{\xi}\right],\left[\Lambda, l_{z}\right],\left[\Lambda, J_{z}\right]$.

Consideremos o operador helicidade formalmente definido pela equação (2.1.13) como sendo igual a:

$$
\Lambda=\left[\vec{p}+\frac{e}{a} \vec{A}\right] \cdot \vec{\Sigma}
$$

Calculemos o comutador:

$$
\begin{gathered}
{\left[\Lambda, S_{3}\right]=\frac{1}{2}\left[p_{x} \Sigma_{1}+p_{y} \Sigma_{2}+\frac{e \phi}{c \tau^{2}}\left(-y \Sigma_{1}+x \Sigma_{2}\right), \Sigma_{3}\right]} \\
\left\lfloor\Lambda, S_{3}\right]=\frac{1}{2}\left[p x_{1}\left[\Sigma_{1}, \Sigma_{2}\right]+p\left[\left[\Sigma_{2}, \Sigma_{3}\right]+e \frac{\phi}{r^{2}}\left(-y\left[\Sigma_{1}, \Sigma_{3}\right]+x\left[\Sigma_{2}, \Sigma_{3}\right]\right)\right]\right.
\end{gathered}
$$

onde usamos a equaçāo (2.1.11) para o potencial vetor $\vec{A}$.

Atraves das definiçöes das matrizes $\Sigma_{1}, \Sigma_{2}$ e $\Sigma_{3}$ dadas respectivamente pelas equagöes $(2.1 .15),(2.1 .16)$ e $(2.1 .21)$ e usando a relagäo de comutaçăo (B-1) conhecida entre as matrizes de Paull podemos facilmente escrever:

$$
\left[\Sigma_{1}, \Sigma_{3}\right]=-2 i \Sigma_{2}
$$

e:

$$
\left[\Sigma_{2}, \Sigma_{3}\right]=2 i \Sigma_{1}
$$

De modo que a equaça $(\mathrm{C}-2)$ se torna:

$$
\left[A_{s} s_{3}\right]=i\left(\vec{\Sigma} \wedge \vec{p}+\frac{e}{c r^{2}} \vec{\Sigma} \cdot \vec{T}\right)
$$

Com $l_{*}$ definido como na equaçäo (B-9) podemos calcular:

$$
\left[\Lambda, l_{z}\right]=\left[p x \Sigma_{1}+m \Sigma_{2}+\frac{e}{c} \frac{\phi}{T^{2}}\left(-y \Sigma_{1}+x \Sigma_{2}\right), x p_{y}-y p_{z}\right]
$$


Usando as relaçóes de comutaşăo conhecidas das equaçōes (B-11) e (B-12) a equacăo (C-6) axima se torma:

$$
\left[\Lambda_{i} l_{z}\right]=\left[p_{x 1}, x\right] \Sigma_{1} p_{y}-\left[p_{y}, y\right] \Sigma_{2} p_{x}+\frac{e \phi}{c r^{2}}\left(-\left[y, p_{y}\right] x_{i} \Sigma_{1}-\left[x_{1} p_{x}\right] y \Sigma_{2}\right)
$$

de onde obtemos:

$$
\left[\Lambda, l_{z}\right]=-\left(\vec{\Sigma} \wedge \vec{p}+\frac{\varepsilon}{c r^{2}} \vec{\Sigma} \cdot \vec{r}\right)
$$

De modo que as equaçöes $(\mathrm{C}-5)$ e $(\mathrm{C}-7)$, implicam no resultado:

$$
\left[A_{2}, J_{z}\right\rfloor=0
$$




\section{Apêndice D}

\section{Discussão dos resultados das referências [5], [6] e [7].}

O objetiwo desse apêndice e comentar os resultados das referências [5], [6] e [7], compará-los com os demais resultados das referências [1], [2] e [4] e com os resultados apresentados nessa tese.

O procedimento limite das referencias [5], [6] e [7], onde se toma um tubo de fluxo magnético de raio $R>0$ (com o campo magnético $\vec{H}$ dado por uma função do tipo $f$ de casca), a fin de se remover a ambiguidade, tomando-se o limite $R \rightarrow 0$ no final dos cálculos, $e$ o mesmo procedimento utilizado pelos autores das referências [2] e [4] e tambén no Capitulo 10 dessa tese.

No entanto, as resultados obtidos por Hagen contém uma dependência no sinal de $s(s= \pm 1)$ que nằ aparece nas soluções dos demais autores citados acima.

Um fato que torna um tanto obscuro os resultado das referéncias [5], [6] e [7] é que $o$ autor, näo encarando o problema como um problema de determinafăo das condiçöes de contorno, não deixa explicito quais condiçôe de contorno suas autohuncoses obedecem dentro daquela familia de condicoes de contorno admissiveis encontratas por Sousa Gerbert ${ }^{[i}$.

Outro fato que chama-nos a atenção é que Hagen obtém soluçōes singulares admissiveis para $-1<\nu=n+\phi<0$ quando $s=+1$ e para $0<\nu<1$ quando $s=-1$.

Se voltarmos a Caṕtulo 2 dessa tese e às referêncías [1], [2], [2] e [4] veremos que só sāo possiveis extensões automadjuntas para $-1<\nu<0$, pois fora desse intervalo o operador hamiltoniana utilizado essencialmente auto-adjunto não admitindo outra condiçăo de contomo senāo a regularidade na origem.

É preciso, entẫo, esclarecermos esses fatos aqui. A explicaçāo para esses fatos está nas diferentes maneiras corno os autores introduzem o spin, ou seja, na forma do operador hamiltoniana. O hamiltoniana usado por Hagen obtido usando-se a decomposiço da equação de Dirux usual de quatro componentes na ausencheia da terceira coordentada espacial em duas equaçó para $s=+1$ e $s=-1^{\left[5, s_{1}, 7\right]}$, enquanto a hamiltoniana das reterencias [1], [2]: [3] e [4] ê a mesma usada nessa tese (veja equação $(2.1 .1)$ ), fornecendo o limite räo relativistico correto. 
Vamos recapitular alguns de nossos resultados importantes para o esclarecimento dos fatos. A hamiltoniana de duas componentes que apresentamos no Capitulo 2 (equaçäo (2.1.10)) ê a seguinte:

$$
H=\sigma_{1} \pi_{z}+\sigma_{2} \pi_{y}+s \sigma_{3} m
$$

$\operatorname{com} s= \pm 1$, onde $\vec{\pi}=\left(\pi_{z} \pi_{y}\right)$ é definido na equaçäo $(2.1 .2)$.

Após passarmos para coordenadas polares, a hamiltoniana da equaçâo (D-1) acima pôde ser crita como:

$$
H(r, \theta)=\left(\begin{array}{cc}
m s & -i e^{-i \theta}\left(\partial_{r}-\frac{i}{r} \partial_{\theta}+\frac{p}{r}\right) \\
-i e^{i g}\left(\partial_{r}+\frac{i}{r} \partial_{0}-\frac{b}{r}\right) & -m s
\end{array}\right)
$$

Para resolvermos o problema de determinaçấ das auto-funfôes mais gerais de H:

$$
H \psi_{ \pm}= \pm|E| \psi_{ \pm}
$$

pudemos tentar uma separaçåo de variáveis do tipo:

$$
\psi_{ \pm}=\left(\begin{array}{l}
\psi_{y}^{1}(r) e^{i n_{1} \theta} \\
\psi_{ \pm}^{2}(r) e^{i r_{2} \theta}
\end{array}\right)
$$

de onde surgin a condição:

$$
n_{2}=n_{1}+1 \text {. }
$$

Pudemos pensar, entăo, em duas possibilidades para as auto-funçố:

$$
\psi_{ \pm}=\left(\begin{array}{c}
\psi_{1}^{1}(r) \\
\psi_{ \pm}^{2}(r) e^{i n}
\end{array}\right) e^{i n \theta}
$$

que tem momento angular total:

$$
j_{z}=n+\frac{1}{2}
$$

e

$$
\psi_{ \pm}^{\prime}=\left(\begin{array}{c}
\psi_{t}^{n}(r) e^{-i z} \\
\psi_{t}^{n}(r)
\end{array}\right) e^{i n \theta}
$$


com momento angular total:

$$
j_{z}=\pi-\frac{1}{2}
$$

Lembremonos que $H$ e $J_{z}$ comutiam.

Como pudemos obter o mesmo valor para o momento angular total da equação (D-6b) fazendo $n \rightarrow n-1$ na equagia (D-5b), claro que as cquacöes para a hamiltonituna $H(x)$ e para as auto-funçöes $\psi_{ \pm}(t)$ obtidas através da equagäa (D5a) sāo equivalentes șs mesmas equaçōes obtidas através da equação (D-6a) pela transformação $\nu \rightarrow \nu-1$, como pode ser facilmente checado.

Assim, escolhendo a auto funçäo da equagäo (D-5a) (sem perda de generali:dade) obtivemos a seguinte equação radial para as anto-funções:

$$
\begin{gathered}
H(r) \psi_{ \pm}(r)= \pm|E| \psi_{ \pm}(r) \\
\left(\begin{array}{cc}
m s & -i\left(\partial_{r}+\frac{\psi+1}{r}\right) \\
-i\left(\partial_{r}-\frac{\psi}{r}\right) & -m s
\end{array}\right)\left(\begin{array}{l}
\psi_{t}^{2}(r) \\
\psi_{ \pm}^{2}(r)
\end{array}\right)= \pm|E|\left(\begin{array}{l}
\psi_{ \pm}^{2}(r) \\
\psi_{ \pm}^{2}(r)
\end{array}\right)
\end{gathered}
$$

de onde encontramos:

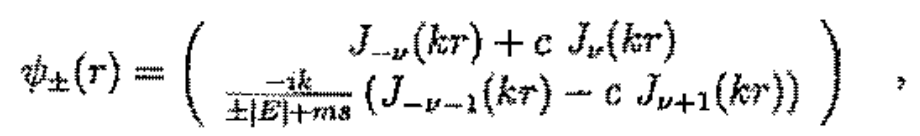

havendo a seguinte relaçäo entre as componentes superior e inferior do espinor de auto-funçöes (veja equaçäo $(2.2 .22)$ ):

$$
\psi_{*}^{2}(k r)=\frac{-l k}{ \pm|E|+m s}\left(\partial_{k r}-\frac{\nu}{k r}\right) \psi_{ \pm}^{1}(k r)
$$

Assim, observando a equaça (D-8) acima, vemos que para os valores de $s$, $s=+1$ ou $s=-1$, o que muda é apenas a proporça entre as componentes superior $\psi_{ \pm}^{1}$ e inferior $\psi^{2}$ das auto-funcōos, dada atraves do fator:

$$
\frac{-15}{ \pm|E|+m s}
$$

não mudando a forma das auto-funçös.

Conforme já analisamos no Capitulo 2 para $y \geq 0$ e $y \leq-1$, apenas as soluçöes regulares na origen sẫo admissiveis, urua vez que as soluções singulares 
na origern nh̆o são localmente normalizäveis (na vizinhança da origem). Para $-1<$ $y<0$, ocorte uma indeterminaçăo, sendo possiveis várias extensôes auto-adjuntas implicando en várias dinâmicas diferentes possiveis, uma vez que equação (D-8) impede a imposiçăo de regularidade na origem simultaneamente para ambas as componentes (superior e inferior) e que as soluçoes singulares säo normalizáveis para esse intervalo de $w$.

A hamiltoniana definida nas referéncias [5],[6] e [7] e que também representa una partínla de Dirac em duas dimensöes sujeita a uraa linha de fluxo magnético na origem tern a forma:

$$
\vec{H}=\sigma_{1} \pi_{1}+s \sigma_{2} \pi_{1}+m \sigma_{3}
$$

$\operatorname{com} s= \pm 1$

Vomos que fazendo-se $s=1$ na equagäo (D-10) e na equacäo (D-1) obtemos a mesma hamiltoniana portanto os resultados obtidos por Hagen para $s=+1$ devem ser, e de fato stu, conforme verificuremos explicitamente a seguir, iguatis aos encontrados nexsa tese (Capítulo 10), e nas referências [2] e [4]. Assim, para a hamiltoniana da exuação (D-10), para $s=+1$, o problema de indeterminacã das condições de contorno será exatarnente o mesmo de que tratamos nessa tese e ocorre para $-1<\nu<0$.

No entanto, para $s=-1$, a hamiltoniana da equagäo (D-10) se torna:

$$
\widetilde{H}=\sigma_{1} \pi_{1}-\sigma_{2} \pi_{1}+m \sigma_{3}
$$

que pode ser escrita ern coordenadas polares como sendo

$$
\widetilde{H}=\left(\begin{array}{cc}
m & -i e^{i \theta}\left(\partial_{r}+\frac{i}{r} \partial_{\theta}-\frac{\phi}{r}\right) \\
-i e^{-i \theta}\left(\partial_{r}-\frac{i}{r} \partial_{\theta}+\frac{\phi}{r}\right) & -m
\end{array}\right) .
$$

Para resolvermos o problema de auto-funçoes:

$$
\bar{H} \Psi_{i}= \pm|E| \Psi_{ \pm s}
$$

podemos tentar a separação de variáveis da equaçă丶 (D-3), o que resulta na condição:

$$
n_{3}=n_{1}-1
$$


Podemos pensar em duas possibilidades para as auto-funçōes:

$$
\Psi_{ \pm}=\left(\begin{array}{c}
\Psi_{ \pm}^{\prime}(r) \\
\Psi_{ \pm}^{2}(r) e^{-i \theta}
\end{array}\right) e^{i n \theta}
$$

mi:

$$
\Psi_{ \pm}^{\prime}=\left(\begin{array}{c}
\Psi_{ \pm}^{\prime 1}(r) e^{i \theta} \\
\Psi_{ \pm}^{\prime}(r)
\end{array}\right) e^{i n \theta}
$$

que não possuem momento angular total $j_{\mu}$ bern definido pois o operador $H$ dado pela equaçäo (D-11) näo comuta com $J_{z}$ como se pode facilmente verificar.

Como podemos obter a mesma auto-função da equação (D-16), fazendo $n \rightarrow$ $n+1$ na equaçäo (D-15), é claro que as equaçóes para a hamiltoniana $\bar{H}(r)$ e para as auto-fungöes $\Psi_{ \pm}(r)$ obtidas através da equaç̃o (D-15) são equivalentes às mesmas equaçôs obtidas através da equayăo (D-16) pela transformaçäo $\nu \rightarrow \nu+1$ (como pode ser faxilmente checado).

Assim, escolhendo a separaçäo de variáveis da exuaçăo (D-15) (sern perda de generalidade), obtemos a seguinte equaçâo radial para as auto-funçōes:

$$
\begin{gathered}
\widetilde{H}(r) \Psi_{ \pm}(r)= \pm|E| \Psi_{ \pm}(r) \\
\left(\begin{array}{cc}
m & -i\left(\partial_{r}-\frac{\nu-\underline{z}}{r}\right) \\
-i\left(\partial_{r}+\frac{z}{r}\right) & -m
\end{array}\right)\left(\begin{array}{c}
\Psi_{ \pm}^{2} \\
\Psi_{ \pm}^{2}
\end{array}\right)= \pm|E|\left(\begin{array}{c}
\Psi_{ \pm}^{2} \\
\Psi_{ \pm}^{2}
\end{array}\right),
\end{gathered}
$$

de onde vem:

$$
\Psi_{ \pm}(r)=\left(\begin{array}{c}
J_{-\nu}+d J_{v} \\
\frac{-i k}{ \pm[\bar{E} \mid+m}\left(-J_{-\nu+1}+d J_{y-1}\right)
\end{array}\right)
$$

com a sezuinte relagăo entre as componentes superior e inferior do expinor de auto-funções:

$$
\Psi_{ \pm}^{2}(r)=\frac{-b}{ \pm|E|+m}\left(\partial_{r}+\frac{\nu}{r}\right) \Psi_{ \pm}^{1}(r)
$$

Analisando a auto-funçāo para $s=-1$ da equasão (D-18) acima vemos que ela é diferente das auto-funçöes da equaç̃o (D-7). 
De modo que, das equaçoe (D-18) e (D-19) vemos que, para $\nu \leq 0$ e $\nu \geq 1$ ape nas as soluçoes regulares na origem säo admiskiveis, uma vez que as soluçōes singulares (na origem) năo são localmente normalizśveis (na vizinhança da crigem).

Para $0<\nu<+1$, a equaça (D-19) impede a impasicäo de regularidade simultaneamente para ambas as componentes (superior e inferior), ocorrendo assim uma indeterminaçäo. De modo que são possíveis extensões antoradjuntas para $H$ quando $0<v<1$ para $s=-1$.

Notamos entäo que, para a hamiltoniana da expressăo (D-1), a indeterninaçäo ocorre sempre no intervalo $-1<v<0$, qualquer que seja o valor de $s(s= \pm 1)$.

No entanto, para a hamiltoniana da equagä́n (D-10) a indeterminaçäo ocorre para o mesmo intervalo $-1<\nu<0$ apenas para $s=+1$, enquanto para $s=-1$ a indeterminaçäo ocome no intervalo $0<y<1$.

Essa é a razäo pela qual os resultados de Hagen ${ }^{[5,6,7]}$ (obtidos através do processo limite do tubo de fluxo de raio $R>0$ ) dependem do valor de $s$ ecorrem para diferentes sinais do fuxo magnético $\phi$.

Notemos também que na referência $[7]$, Hagen conclui a possibilidade do soluçio singular para a componente superior quando $s=+1$, para $n>0$ e $4<0$ (veja equaçăo (3.15b) da referência [7]), o que coincide com o resultado que nó apresentamos na Tabela 12 do Capítulo 10 e com os resultados das referências [2] e [4]. Tal fato é bastante esperado una vez que as hamiltonianas usadas por Hagen (equaça D-10) e pelos demais autores (equanga (D-1)) coincidem para $s=+1$.

No entanto a outra soluçäo singular obtida para a componente superior quando $s=-1$, que de acordo com a equaçäo (3.15a) đa referência $[7]$, ocorre para $n<0$ $e \varphi>0$, não aparece nas referèncias [2] [4] e também näo aparece nessa tese, pois nesse caso nẩo corre identidade entre os hamiltonianas utilizados por Hagen e pelos demais antores, sendo esperado, portanto, previsöes diferentes para $s=-1$.

No entanto, podemos observar que as hamiltonianas das equações (D-1) e (D-10) estäo relacionadas pela transformacăo:

$$
V H V=H
$$

onde:

$$
V=e^{i \frac{w_{2} a_{1}}{2}}=\left(\begin{array}{cc}
0 & \frac{1}{\sigma} \\
i & 0
\end{array}\right)
$$


De modo que, a equivalencia unitária da equaçäo (D-20) implica que as autofunçồ das equacōes (D-3a) e (D-13) estäo relacionsulas por:

$$
\begin{aligned}
& \Psi_{ \pm}(r)=V^{\dagger} \psi_{ \pm}^{\prime}(r), \\
& \Psi_{ \pm}^{\prime}(r)=V^{\dagger} \psi_{ \pm}(r) .
\end{aligned}
$$

Utilizando as equaçöes (D-5a), (D-6a), (D-15) e (D-16), vemos que as equacôes aciron implicam em:

$$
\begin{gathered}
\Psi_{ \pm}^{1}(r)=-i \psi_{ \pm}^{\prime 2}(r) \quad \text { e } \quad \Psi_{ \pm}^{2}(r)=-i \psi_{ \pm}^{1}(r) \\
\Psi_{ \pm}^{\prime 1}(r)=-i \psi_{ \pm}^{2}(r) \quad \text { e } \quad \Psi_{ \pm}^{f 2}(r)=-i \psi_{ \pm}^{3}(r) .
\end{gathered}
$$

Podemos chesar as relacoes acima rapidamente, notando que, fazendo $y \rightarrow$ $y-1$ na ecuação (D-7b) para $\psi_{*}(r)$, devemos obter a expressăo para $\psi_{ \pm}^{*}(r)$. Trocando a componente superior pela inferior e vice-versa no espinor assim obtido, obtemos $\Psi_{ \pm}(r)$, oque está de veordo com as equaçoes (D-22a) e (D-22b).

Num comunicado informal Hagen disse que os procedimentos limite utilizados nas referencias [2] e [4] nầ são os "mais corretos" e que a análise perfata é a apresentada nas referéncias [5], [6] e [7].

Refazendo os cálculos das referências [0], [6] e [7], vemos que, embora näo haja noda de errado com esses calculos, eles simplesmente parecem ser uma outra maneira, talvez mais complicada, de se calcular os limites de $h \rightarrow 0$.

Iniciamos notando que as condicoes impostas a componente superior (do pinor de duas componentes da auto-fungino), através das equacöos (3.8a) e (3.8b) da referência [7], que correspondern respectivamente à continuidade da funçäo e salto da derivada primeira en $*=R$, são equivalentes à imposição da continuidade para ambas as componentes superior inferior (condição usada nas referêtucias [2] Q [4] e nas equaços $(10.2 .16)$ e (10.2.17) dessa tese) conforme podemos imediatamente verificar considerando as equaçóes (D-7) para $s=+1$ e (D-18) para $s=-1$.

Impondo essas condiçoes a componente superior, tomando o limite $R \rightarrow 0$, lembrando do comportamento na origem das funçô de Bessel dada pela equaçăo $(5.5 .6)$, e fazendo a unális do termo dominante na equaçăo (3.10) da referância [7] chega-se de fato na condição da equaçäo (3.11). Essa condição parece não surgí nos calculos da Seçäol0.2 dessa tese.

No entanto, se nas lembrarmos que os cálculas da referência [7] e os nossos devem coincidir para $s=+1$ e que, no nosso caso, nơs jâ sabemos (pela analibe 
prévia da forma das auto-funçö́s da equaçăo (D-7)) que o intervalo crítico para $\nu$ é ] $-1,0$, vemos que para $n>0$ a equaşa (3.11) é automaticamente satisfeita, ou seja, o primeiro termo da equagão (3.10) se anula e sornos obrigados, em nossos cálculos, a tornar outros termos de ordem imediatamente superior $\mathrm{em} k r$, chegando a equação (10.2.19), que é exatamente a condiçâa ds equaçäo (3.13) (para $y:]-1,0[$ e $s=+1$ ) da referência [7], para que haja solusāo singular para a componente superior.

Do mesmo modo, note que, ainda para $s=+1$, a condiçäo da equação (3.11) da referência [7], näo ế satisfeita para o intervalo criticto $-1<v<0$ com $n<0$ (o que significa que devemos ter a solução regular) e por isso em nossos cálculos a análise do termo dominante conduz à soluçâno regular conforme mostram nossas equaços $(10.2 .20)$ e a segunda equacão da Tabela 12.

Para $s=-1$, como dissemos, é possivel fazermos a equivalència entre os resultados das referências [2], [4] e os nossos com os obtidos por Hagen, e utilizando o mesmo procedimento que fizemos no Capitulo 10, podenos chegar rapidamente âo resultado da equaçăo (3.15a) da referência [7]. Como nở já sảbemos fatravés de nossa análise prévia das soluçoes da equą̧ăo (D-18)], que o intervalo crítico para $\nu$ quando $s=-1$ é 0,1 , a análise do termo dominante na equação (3.10) (levando em conta que $v$ está nesse intervalo), nos leva à aquação (3.11) da referëncia [7] satisfeita apenas se $n<0$, o que significa $\phi>0$ (para $v ; 0,10$, que corresponde exatamente ao resultado da exuaģāo (3.15a) de Hagen.

Portanto, nossa análise "mais simplificada " dos termos dominantes é equivalente análise feita por Hagen, conduzindo as mesmos resultados, conforme se pôde verificar.

A análise feita na referêneia [7], embora correta, nào stá completa, uma vez que as auto-funçōes näo estảo completamente determinadas unicamente pelas equaçôes $(3.15 \mathrm{a})$ e $(3.15 \mathrm{~b})$, sendo necessấria além da análise da componente superior a análise do comportamento da componente inferior através das equaçoes (D-8) para $s=+1$ e (D-19) para $s=-1$, que relacionam a componente inferior com a superior.

Efetuando-se essa analise para $s=+1$, a equação (D-8) fornece componente inferior regular na situação da equação (3.15b) satisfeita, ou seja, quando a componente superior 6 singular (para $y:]-1,00$, e componente inferior singular (e normalizável na origem) quando a condigăo (3.15b) näo é satisfeita, ou seja, quando a componente superior é regular (para $v:]-1,0[$ ). De forma que as soluçôs säo as apresentudas na Tabela 12 dessa tese, que correspondem a extensöes allto-adjuntas para $H$ com o parâmetro $\theta$ di equação (10.1.1) igual a 
$-\operatorname{sgn}(\phi) \frac{\pi}{2}$, ou o parầmetro $\delta$ da equação (5.3.30) igual a 0 e $\frac{\pi}{2}$.

Fora do intervalo $v:]-1,0[$, as úmicas soluçôes possíveis são as regulares na origenn, como já discutimos anteriornenté.

Efetuando-se a mesma análise para $s=-1$ (para $z: 0,10$ ), a equação (D-19) implicará exn componente inferior regular quando a condição (3.15a) da referência [7] é satisfeita (isto é, quando a componente superior é singular) e componente inferior singular e normalizável na origem qquando a condição (3.15a) não 6 satisfeita (isto é, quando a componente superior

Assim para $s=-1$, o procedimento limite do tubo de fluxo para a hamiltoniana da equaçẫo (D-10) quando $\nu$ : 10,1, conduz às soluçôes:

$$
\Psi_{ \pm}(k r)=\left(\begin{array}{c}
J_{-\nu}(k r) \\
\frac{i k}{ \pm[E \mid+\dot{m}} J_{-\nu+1}(k r)
\end{array}\right), \text { para } n<0, \phi>0
$$

e

$$
\Psi_{ \pm}(k r)=\left(\begin{array}{c}
J_{\nu}(k r) \\
\frac{-i k}{ \pm|E|+m} J_{\nu-1}(k r)
\end{array}\right) \text {, para } n>0, \phi<0
$$

Para $s=-1$ e fora do intervalo crítico $v:] 0,1[$, como dissemos anteriormente,

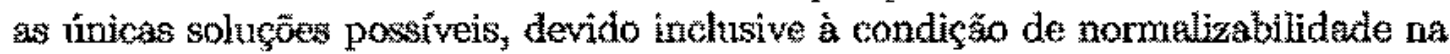
origem, são as soluçöes rẹgulares na origem. 


\section{Apêndice $\mathrm{E}$}

Atuação do operador $H$ nas auto-funçöes do operador $\Lambda$ definidas no domínio $D_{5}(H, \Lambda)$.

Podernos verificar que, além do operador $\Lambda$ näo deixar o domínio $D_{\delta}(H, \Lambda)$ definido na Seçăo 5.4 invariante, isto é:

$$
A D_{s}=D^{\prime} / D^{\prime} \frac{1}{F} D_{5}
$$

também o operador $H$, atuando em certas funçōs do dominio $D_{8}\left(H_{1} \Lambda\right)$, gera funcoues fora desse domínio, isto é:

$$
H D_{b}=D^{\prime \prime} / D^{H} D_{b}
$$

Vejamos como isso acontece, deixando $H$ atuar numa auto-fungäo de $\Lambda$, da forma da equagāo (5.6.1), que pode também ser escrita na forma da equaça (2.3.4), pertencente ao domínio $D_{\delta}(H, \Lambda)$, sujeita às condif̧o de contorno que produairam as resultados da Tabela 3. Assim:

$$
\Phi=H \psi_{w}=\left(\begin{array}{cc}
h+m \sigma_{3} & 0 \\
0 & h-m \sigma_{3}
\end{array}\right)\left(\begin{array}{c}
\psi_{ \pm}^{(1)} \\
\psi_{ \pm}^{(2)}
\end{array}\right)=\left(\begin{array}{c}
\left(h+m \sigma_{3}\right) \psi_{ \pm}^{(1)} \\
\left(h-m \sigma_{3}\right) \psi_{ \pm}^{(2)}
\end{array}\right)
$$

Invocando os resultados das equaçöes (2.3.5) e (2.3.6) e sendo $\psi$ ta da forma da equação (5.6.1), de modo que $\psi_{ \pm}^{(1)}$ é dado por $(2.3 .15)$ e $\psi^{42}$ é dado por $(2.3 .16)$, encontramos:

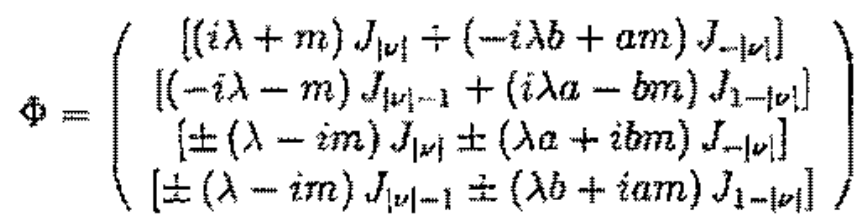

É fácil vermos que, se the satisfaz as condiçöes de contomo das equaçôes $(5.4,2)$ e (5.4.3), ou seja, se as constantes a $a$ säo dadas de acordo com a Tabela 3 , então $\Phi \notin D_{b}(H, \Lambda)$, a menos que o parâmetro $\delta$ da extensäo assuma os valores $\delta=0 \mathrm{e}$ $\bar{b}=\frac{\pi}{2}$. 


\section{REFERENCIAS}

[1] - F. A. B. Coutinho e J. Fernando Perez, Phys. Rev, D 49, 2092 (1994)

[2] - F. A. B. Coutinho e J. Fernando Perez, Phys. Rev. D 48, 932 (1993)

[3] - Ph. de Souza Gerbert, Phys. Rev. D 40, 1346 (1989)

[4] - M. G. Alford, J.March - Russell e F. Wilczec, Nuclear Phys. B328, 140 (1989)

[5] - C. R. Hagen, Phys, Rev, Sett 64, 503 (1990)

[6] - C. R. Hagen, Phys. Rev. D 48, 3935 (1993)

[7] - C. R. Hagen, International Journal of Modern Physics A, (Vol. 6), 3119 (1991)

[8] - F. A. B. Coutinho, Y. Nogami e J. Fernando Perez, J. Phys. A : Math Gen 32 (1999)

[9] - F. A. B. Coutinho, Y. Nogami e J. Fernando Perez, J. Phys. A : Math Gen 30, 3937 (1997)

[10] - M. Abramowitz e Irene A. Stegun, Handbook of Mathematical Functions, (Dover Publications, New York (1968))

[11] - M. Reed e B. Simon, Methods of Modern Mathematical Physics, (Academic, New York 1975/1980), Vols. I and II

[12] - Thomas F. Jordan, Linear Operators for Quantum Mechanics (John Wiley \& Sons, New York (1969))

[13] - M. Carreau, E. Farhi e S. Gutmann, Phys. Rev. D 42, 1194 (1990)

[14] - S. Albeverio, L. Dabrowski e P. Kurasov, Lett. Math. Phys 4533 (1998) 
[15] - Y. Aharonov e D. Bohm, Phys. Rev. 119, 485 (1959)

[16] - W. Ehrenberg e R. W. Siday, Proc. Phys. Soc. London B 62, 8 (1949)

[17] - Y. Aharonov e A. Casher, Phys. Rev. Lett. 53, 319 (1984)

[18] - M. G. Alford e F. Wilczek, Phys. Rev. Lett. 623, 1071 (1989)

[19]-M. G. Alford, J. March-Russell e F. Wilczek, Nucl. Phys. B328, 140 (1989)

[20] - Ph. Gerbert e R. Jackiw, Commun. Math. Phys. 124, 229 (1989)

[21] - Y. H. Chen, F. Wilczek, E. Witten e B. I. Halperin, Int. J. Mod. Phys. B 3, 1001 (1989)

[22] - Ph. Gerbert e R. Jackiw. Commun. Math. Phys. 124, 229 (1989)

[23] - E. Zeidler, Applied Functional Analysis, Springer- Verlag, NewYork, Inc. (1995)

[24] - I. Stakgold, Green's Functions and Boundary Problems, John Wiley \& Sons, Inc (1998)

[25] - S. Flugge, Practical Quantum Mechanics, Springer-Verlag, NewYork, 1974

[26] - S. Albeverio, F. Gesztesy, R. Hoegh-Krohn, H. Holden, Solvable Models in Quantum Mechanics, pg. 357, Springer- Verlag, NewYork, Inc. (1988)

[27] - N. I. Akhiezer, I. M. Glazman, Theory of Linear Operators in Hilbert Space, Dover Publications, pg. 91, Inc., 1993

[28] - M. Reed e B. Simon, Methods of Modern Mathematical Physics, (Academic, New York 1975/1980), pg. 140, Vols.II 